\title{
Numerical Study of Laminar Free-Shear-Layer Stability in Wall Proximity and in the Presence of Cross-Stream Thermodynamic Property Gradients
}

by

\author{
Aneesh John \\ A Thesis submitted to \\ the Faculty of Graduate and Postdoctoral Affairs \\ in partial fulfilment of \\ the requirements for the degree of \\ Master of Applied Science
}

Ottawa-Carleton Institute for

Mechanical and Aerospace Engineering

Department of Mechanical and Aerospace Engineering

Carleton University

Ottawa, Ontario, Canada

(C)August 2012

Aneesh John 
Library and Archives

Canada

Published Heritage

Branch

395 Wellington Street

Ottawa ON K1A ON4

Canada
Bibliothèque et

Archives Canada

Direction du

Patrimoine de l'édition

395 , rue Wellington

Ottawa ON K1A ON4

Canada
Your file Votre référence

ISBN: 978-0-494-93474-6

Our file Notre référence

ISBN: $978-0-494-93474-6$
NOTICE:

The author has granted a nonexclusive license allowing Library and Archives Canada to reproduce, publish, archive, preserve, conserve, communicate to the public by telecommunication or on the Internet, loan, distrbute and sell theses worldwide, for commercial or noncommercial purposes, in microform, paper, electronic and/or any other formats.

The author retains copyright ownership and moral rights in this thesis. Neither the thesis nor substantial extracts from it may be printed or otherwise reproduced without the author's permission.
AVIS:

L'auteur a accordé une licence non exclusive permettant à la Bibliothèque et Archives Canada de reproduire, publier, archiver, sauvegarder, conserver, transmettre au public par télécommunication ou par l'Internet, prêter, distribuer et vendre des thèses partout dans le monde, à des fins commerciales ou autres, sur support microforme, papier, électronique et/ou autres formats.

L'auteur conserve la propriété du droit d'auteur et des droits moraux qui protege cette thèse. $\mathrm{Ni}$ la thèse ni des extraits substantiels de celle-ci ne doivent être imprimés ou autrement reproduits sans son autorisation.
In compliance with the Canadian Privacy Act some supporting forms may have been removed from this thesis.

While these forms may be included in the document page count, their removal does not represent any loss of content from the thesis.
Conformément à la loi canadienne sur la protection de la vie privée, quelques formulaires secondaires ont été enlevés de cette thèse.

Bien que ces formulaires aient inclus dans la pagination, il n'y aura aucun contenu manquant. 
Copyright (C)

2012 - Aneesh John 


\section{Abstract}

This thesis investigates the stability characteristics of laminar planar shear layers, including the effect of proximity of the shear layer to a nearby wall and the effect of cross-stream property gradients for fluids in the thermodynamic supercritical state. The results of a series of numerical studies are presented for the stability characteristics of a shear layer in close proximity of a no-slip wall. The shear layer is formed by the merging of two boundary-layer flows separated by a thin splitter plate. The Reynolds number at the splitter-plate trailing-edge, the velocity ratio of the high-and low-speed streams, and the proximity of the shear layer to the no-slip wall are systematically varied to investigate the effects of these parameters on the development

of the shear layer. Sensitivity to wall-proximity is clearly observed and an explanation is given based on the interaction with the wall of the shed vortices that form in the unstable free shear layer. Numerical simulations results are presented on the stability characteristics of free shear layers in supercritical fluids with cross-stream property gradients. The results are compared to the stability of free shear layers of subcritical single-phase fuids with matching Reynolds number and velocity ratio. In the case with supercritical working fluid, the free shear layer deformed by the Kelvin Helmholtz instability mode is observed to produce body forces that create an effective Richardson number sufficiently high to sustain the Holmboe instability mechanism. This Holmboe instability produces thin Holmboe vortices that co-exist with the KelvinHelmholtz vortices. Three dimensional instability that follows these instability modes 
is observed to have a reduced dominant spanwise wavelength when the working fluid is at a supercritical state with cross-stream property gradients. 


\section{Acknowledgments}

First and foremost I would like to thank my thesis supervisor, Dr Metin Yaras, for his guidance and support during my research. His dedication to my work through his research advice, his guidance in our group meetings, and his feedback in my numerous thesis revisions enabled me to complete this thesis and improve the standard of my technical communication.

I would also like to extend my sincerest gratitude to my colleague and friend Joshua Brinkerhoff for all his guidance and encouragement during the last few years. As a new researcher in the M.A.Sc. program, Joshua taught me the basics of engineering research and several technical aspects of research into computational fluid dynamics. His spiritual support and guidance also gave me the strength to overcome many personal difficulties and challenges I faced during my journey to complete my thesis. Furthermore, I want to acknowledge Chukwudi Azih, Nikunj Dave, Hristo Valtchanov, Mustafa Mogri, and Majed Abu-Sharar for their support, feedback, and technical advice during my research. Their support was invaluable for overcoming many technical challenges I faced during my research.

I want to thank my family including my father, John, my mother, Annie, and my brother, Praveen, for their encouragement, love and support during the completion of my Masters Degree.

Finally, I want to thank Carleton University for funding of my research and allowing me to use its computing resources, without which this research would not 
be possible. I want to also thank the computing resources technical staff, especially Neil McFadyen, for the numerous times he helped me overcome computing problems during my research. 


\section{Table of Contents}

Abstract $\quad$ iv

$\begin{array}{ll}\text { Acknowledgments } & \text { vi }\end{array}$

Table of Contents viii

Nomenclature $\quad \mathbf{x x}$

1 Introduction 1

1.1 Motivation .......................... 1

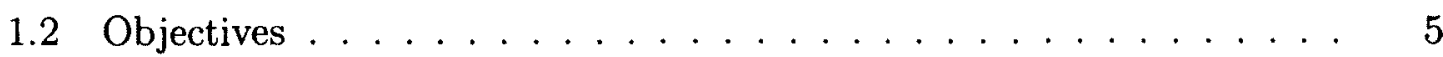

1.3 Approach ....................... 5

2 Literature Survey $\quad 7$

2.1 Separation Bubbles on Axial Turbine Blades . . . . . . . . . 7

2.2 Attached Boundary Layer Stability . . . . . . . . . . . 9

2.2.1 Primary Instability Mechanism . . . . . . . . . . . . . . 10

2.2.2 Secondary Instability Mechanism . . . . . . . . . . . . 15

2.2.3 Breakdown into Turbulence . . . . . . . . . . . 18

2.3 Free and Separated Shear Layer Stability . . . . . . . . . . . . . . 19

2.3.1 Primary Instability Mechanism . . . . . . . . . . . . 20

2.3.2 Secondary Instability Mechanism _. . . . . . . . . . . 24 
2.3.3 Breakdown into Turbulence . . . . . . . . . . . 25

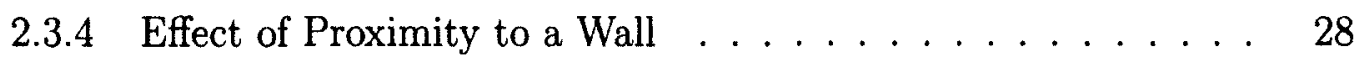

2.3.5 Effect of Cross-Stream Property Gradients . . . . . . . . . . 33

2.4 Thermally Supercritical Flows . . . . . . . . . . . . . . . . 39

3 Computation Method $\quad 42$

3.1 Overview . . . . . . . . . . . . . . . . . . . 42

3.2 Computational Method . . . . . . . . . . . . . . . . . 43

3.2.1 Governing Equations of Fluid Flow . . . . . . . . . . 43

3.2.2 Discretization of the Partial Differential Equations . . . . . . 44

3.2 .3 Solution Algorithm . . . . . . . . . . . . 47

3.3 Computational Setup . . . . . . . . . . . . . . . . . . . . . . 49

3.3.1 Laminar Planar Shear Layer in Proximity to a Wall . . . . . 49

3.3.1.1 Domain Geometry . . . . . . . . . . . . . . . 49

3.3.1.2 Spatial Grid . . . . . . . . . . . . . 50

3.3.1.3 Time-Step Size and Simulation Duration _.... 53

3.3.1.4 Test Matrix . . . . . . . . . . . . . 53

3.3.2 Laminar Planar Free-Shear Layer with Thermodynamic Property Gradients . . . . . . . . . . . . . . 5 55

3.3.2.1 Domain Geometry . . . . . . . . . . . . 55

3.3.2.2 Spatial Grid . . . . . . . . . . . . . 57

3.3.2.3 Time-Step Size and Simulation Duration . . . . . . 58

3.3.2.4 Test Matrix . . . . . . . . . . . . . . . . 59

3.4 Potential Flow Analysis Methods . . . . . . . . . . . . 60

3.4.1 Steady Potential Flow Analysis . . . . . . . . . . . 60

3.4 .2 Transient Potential Flow Analysis . . . . . . . . . . . . 64 
4 Effect of Wall Proximity on the Stability of Laminar Planar Free $\begin{array}{ll}\text { Shear Layers } & \mathbf{6 7}\end{array}$

4.1 Instability Development in the Splitter-Plate Boundary Layer . . . 68

4.2 Instability Development in the Planar Free Shear Layer . . . . . . . 72

4.2.1 Effect of Wall Proximity on the Instantaneous Flow Field . . 72

4.2.2 Effect of Wall Proximity on the Time-Averaged Flow Field . . 78

4.2.3 Effect of Wall Proximity on Transition Onset . . . . . . . 81

4.2.4 Effect of Wall Proximity on the Frequency of Vortex Shedding 83

4.3 Linear Stability Analysis . . . . . . . . . . . . . . . 86

4.4 Transient Potential Flow Analysis . . . . . . . . . . . . . . . 89

4.5 Steady Potential Flow Analysis _ . . . . . . . . . . . . 90

5 Effect of Supercritical Thermodynamic State on Free Shear Layer Development $\quad 93$

5.1 Summary of Flow Configurations $\ldots \ldots \ldots \ldots$

5.2 Instability Development in the Subcritical Shear Layer $\ldots \ldots \ldots$

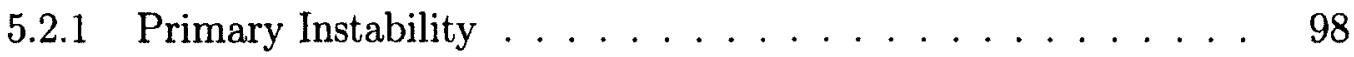

5.2 .2 Secondary Instability . . . . . . . . . . . . . . 102

5.3 Instability Development in the Supercritical Shear Layer . . . . . 104

$5.3 .1 \quad$ Primary Instability $\ldots \ldots \ldots \ldots \ldots$

$5.3 .2 \quad$ Secondary Instability . . . . . . . . . . . . . 110

6 Conclusions

$\begin{array}{ll}\text { List of References } & 114\end{array}$

Appendix A Supercritical Water Thermodynamic Table Validation 127

A.1 NIST and IAPWS Water Property Databases . . . . . . . . . 128

A.2 IAPWS Water Property Formulations $\ldots \ldots \ldots \ldots$ 
A.3 Comparison of Thermodynamic Properties . . . . . . . . . . 132

A.4 Sizing of the Thermodynamic Grid . . . . . . . . . . . 139

A.5 Thermodynamic Grid Convergence Analysis . . . . . . . . . . . 143

$\begin{array}{ll}\text { Appendix B Data Analysis Procedures } & 146\end{array}$

B.1 Overview . . . . . . . . . . . . . . . . . 146

B.2 Selection of Similarity Parameters . . . . . . . . . . 147

B.3 Calculation of Flow Statistical Quantities . . . . . . . . . . . . 148

B.4 Calculation of Flow Differential Parameters . . . . . . . . 150

B.5 Calculation of Flow Integral Parameters $\ldots \ldots \ldots \ldots$

B.6 Spectral Analysis . . . . . . . . . . . . . . . . . 152

B.7 Linear Stability Analysis . . . . . . . . . . . . . . . 153

B.8 Grid Convergence Study . . . . . . . . . . . . . . . . . . . 154 


\section{List of Figures}

1.1 (a) Diagram of a laminar separation bubble on a turbine blade (adapted from Brinkerhoff, 2012). (b) Simplified flow geometry used to investigate the effect of wall proximity on the

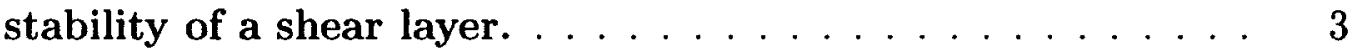

1.2 (a) Nuclear fuel rod in a fuel bundle (adapted from Hansen and Owen, 2008). (b) Shear layer development (shown in green/yellow) in flow through fuel bundle (adapted from Yan and Karoutas, 2011). (c) Simplified flow geometry used to investigate the effect of cross-stream property variations on shear layers composed of supercritical fluid. . . . . . . . . 4

2.1 Schematic of a laminar separation bubble (Brinkerhoff, 2012). 9

2.2 Schematic of stability "thumb plots" for velocity profiles with

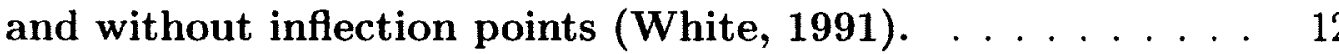

2.3 (a) Experimental measurements and theoretical prediction of T-S wave frequency (Schubauer and Skramstad, 1947). (b) Experimental (symbols) and theoretical (lines) mode shapes (Reed et al., 1996). . . . . . . . . . . . . . . . . . . . . . 13

2.4 (a) Amplification rate and (b) frequency distribution of Tollmien-Schlichting waves for Blasius boundary layer as predicted by linear stability theory (adapted from Mack, 1984). 


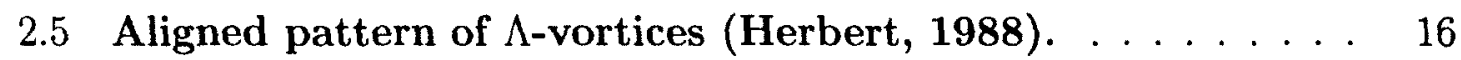

2.6 The development of a hairpin vortex (Smith et al., 1991) . . 17

2.7 The structure of a turbulent spot (Henningson et al., 1987). Coordinate axes are normalized by initial boundary layer momentum thickness, $\delta_{\mathrm{o}} \ldots \ldots \ldots \ldots \ldots \ldots$

2.8 Inviscid stability characteristics of shear layers: $(a)$ an inviscidly stable velocity profile; $(b)$ an inviscid unstable profile. . 21

2.9 Roll-up into Kelvin-Helmholtz vortices (adapted from Drazin

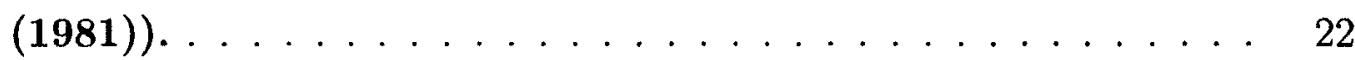

2.10 Stages of the pairing instability illustrated through spanwise vorticity isocontours, where $\lambda_{x}$ is the streamwise wavelength

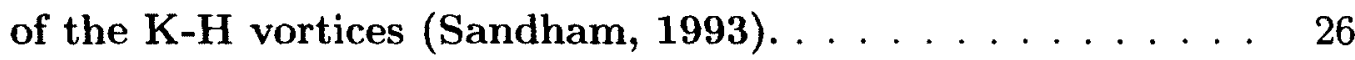

2.11 Development of streamwise vortices (shown using streamwise vorticity contours) in the stream-normal $(y-z)$ plane (adapted

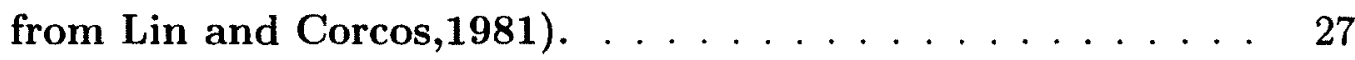

2.12 Streamwise vorticity patterns for (a) type $\mathbf{A}$ and (b) type $B$ instabilities (Baily et al., 2002). . . . . . . . . . . . .

2.13 The development of the streamwise velocity fluctuation profile during the transition of a boundary layer velocity profile to a separated shear layer velocity profile (Dovgal et al., 1993). 29

2.14 Streamwise perturbation velocity profile (u) profiles for (a) $\mathrm{y}_{\mathrm{s}} / \delta_{1}^{*}=0.70$ (b) $\mathrm{y}_{\mathrm{s}} / \delta_{1}^{*}=\mathbf{0 . 5 5}$. Wall-normal height ( $\mathrm{y}$ ) is normalized by shear layer displacement thickness $\left(\delta_{1}^{*}\right)$ (Rist and

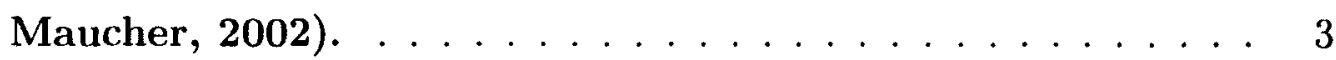

2.15 Scaling relation for the most amplified frequency in the separation bubble (Diwan and Ramesh, 2009). 
2.16 Stability plots for the Taylor Goldstein equation showing contours of unstable waves. Region bounded by $\alpha c_{i}=0.25$ (coloured in blue) are prone to the Holmboe instability (Lawrence et al., 1991) . . . . . . . . . . . . . . . . . . 35

2.17 (a) Schematic showing the formation of Holmboe vortices (Lawrence et al., 1991). (b) Holmboe vortices shown through flood plots of spanwise vorticity where density variation is shown using contour lines (reproduced from Carpenter et al., 2010). . . . . . . . . . . . . . . . .

2.18 Streamwise vortices in a shear layer with cross-stream property gradients accompanying (a) K-H vortices (b) Holmboe vortices (red: positive-sense streamwise vorticity; blue: negative-sense streamwise vorticity)(Smyth, 2005). . . . . .

2.19 The variation of density $(\rho)$, dynamic viscosity $(\mu)$, thermal conductivity $(k)$ and isobaric specific heat capacity $\left(c_{p}\right)$ for water at the critical pressure at $P_{c}=22.1 \mathrm{MPa}($ NIST, 2011). 40

3.1 Setup of control volumes and elements in ANSYS CFX (adapted from Balzek, 2001) . . . . . . . . . . . . .

3.2 Schematic illustrating the $\mathrm{W}$-cycle used as the inner iteration loop by the multigrid scheme in ANSYS CFX. Integers refer to the number of solutions performed at each grid level. Reproduced from McAuliffe (2007) . . . . . . . . . . . . . . 48

3.3 Schematic of the two-dimensional computational domain for the simulations on wall-proximity effects (not to scale). . . 
3.4 Node spacing in the (a) streamwise and (b) wall-normal directions (solid line: mixing region; dashed line: splitter plate

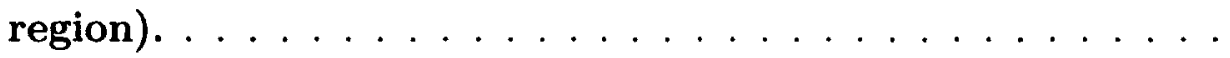

3.5 Temporal variation of circulation calculated within a region near the trailing edge of the splitter plate. . . . . . . . . . 54

3.6 Schematic of the computational domain for the laminar free shear-layer simulations with spatial gradients in supercritical

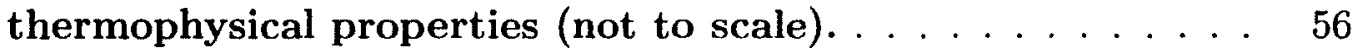

3.7 Node spacing in the (a) streamwise, (b) plate-normal

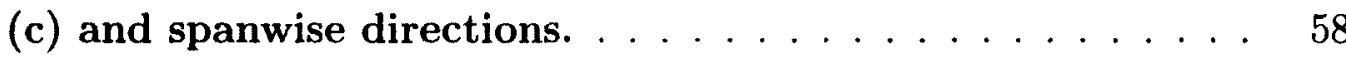

3.8 Vortex patterns for steady potential flow studies for (a) flat vortex sheet (b) streamwise-wavy vortex sheet (c) point vortices and (d) Rankine vortices (blue: negative vorticity; red: positive vorticity; line shows the position of the wall). . . .

3.9 Schematic diagram of the initial setup of the transient potential flow vortex element model. . . . . . . . . . . . . . .

4.1 Displacement thickness, momentum thickness and shape factor growth in the splitter-plate boundary layer for (a) $\operatorname{Re}_{\theta_{\mathrm{s}}}=320$ and (b) $\operatorname{Re}_{\theta_{\mathrm{s}}}=108$.

4.2 Development of the perturbation mode shapes in the splitter

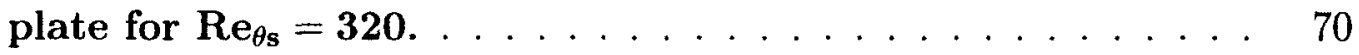

4.3 Streamwise growth of streamwise perturbation velocity magnitude in the splitter-plate boundary layer at $\mathrm{y} / \theta_{\mathrm{s}}=1 . \ldots$

4.4 Instantaneous distribution of spanwise vorticity component for one cycle of vortex-shedding for $\operatorname{Re}_{\theta s}=320, R=1.88$, $\mathrm{y}_{\mathrm{s}} / \theta_{\mathrm{s}}=\mathbf{1 4 . 5}$. 
4.5 Instantaneous distribution of spanwise vorticity component for $\boldsymbol{R e}_{\theta \mathbf{s}}=\mathbf{3 2 0}$ and $\mathbf{R}=\mathbf{1 . 8 8} \ldots \ldots \ldots \ldots \ldots \ldots$

4.6 Instantaneous distribution of spanwise vorticity component for $\operatorname{Re}_{\theta \mathbf{s}}=\mathbf{1 0 8}$ and $R=1.88 \ldots \ldots \ldots \ldots \ldots \ldots$

4.7 Instantaneous distribution of spanwise vorticity component for $\operatorname{Re}_{\theta \mathrm{s}}=\mathbf{3 2 0}$ and $\mathbf{R}=\mathbf{1 . 2 2} \ldots \ldots \ldots \ldots \ldots \ldots$

4.8 RMS of the wall-normal component of perturbation velocity (v) at $y / \theta_{\mathrm{s}}=0$ for (a) $\operatorname{Re}_{\theta \mathrm{s}}=320$ and $\mathrm{R}=1.88$, (b) $\operatorname{Re}_{\theta \mathrm{s}}=108$ and $R=1.88$, and (c) $R_{\theta_{s}}=320$ and $R=1.22 \ldots \ldots 77$

4.9 Time-averaged streamwise velocity for $\mathbf{R e}_{\theta \mathrm{s}}=\mathbf{3 2 0}$ and $\mathbf{R}=1.88 .79$

4.10 Time-averaged streamwise velocity for $R_{\theta_{s}}=108$ and $R=1.88 .79$

4.11 Time-averaged streamwise velocity for $\mathbf{R e}_{\theta \mathrm{s}}=\mathbf{3 2 0}$ and $\mathbf{R}=1.22 .80$

4.12 Computed $(\mathbf{R}=1.88)$ and predicted values of transition onset Reynolds number.

4.13 Sample illustration of the control volume used for monitoring

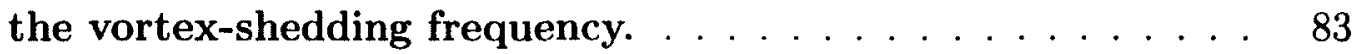

4.14 Variation of vortex shedding frequency with wall proximity. . 84

4.15 Time-mean and perturbation velocity profiles for $\mathbf{R e}_{\theta \mathbf{s}}=\mathbf{3 2 0}$, $R=1.88$ at two wall proximities.

4.16 Shear layer development predicted through a transient potential-flow analysis for (a) free-shear-layer, (b) far wall-proximity $\left(y_{s} / y_{p}=1000\right)$, and (c) close wall-proximity $\left(y_{s} / y_{p}=250\right)$ where $\mathscr{T}$ is one period of vortex shedding. . . .

4.17 Wall-normal distribution of streamwise velocity under locations of maximum circulation for (a) flat vortex sheet, (b) streamwise-wavy vorticity pattern, (c) point vortex, and (d) Rankine vortex. 
5.1 Splitter-plate high-speed-stream boundary layer velocity profile at the trailing edge $\ldots \ldots \ldots \ldots \ldots$

5.2 Cross-stream variation in temperature $(T)$, isobaric heat capacity $\left(\mathbf{c}_{\mathbf{p}}\right)$, density $(\rho)$ and viscosity $(\mu)$ in the supercritical

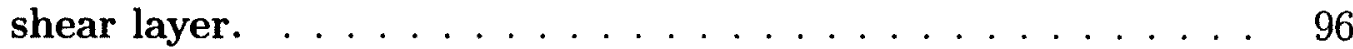

5.3 Streamwise variation of free-shear-layer momentum thickness. 98

5.4 Instantaneous isocontours of spanwise and streamwise vorticity for one vortex-shedding cycle $(\mathscr{T})$ of the subcritical shear layer (green: spanwise vorticity $\left(\omega_{z}=400 s^{-1}\right)$; red and blue: streamwise vorticity of counter-rotating vorticity $\left.\left(\omega_{\mathbf{x}}= \pm 40 \mathrm{~s}^{-1}\right)\right)$.

5.5 Power spectral density of $y$-component velocity fluctuation in the subcritical shear layer at $\mathbf{y} / \theta_{\mathbf{f}}=0 \ldots \ldots \ldots \ldots$

5.6 Subcritical shear layer flood plots at $z^{+}=0$ of (a) instantaneous spanwise vorticity distribution and (b) time-averaged streamwise velocity distribution. . . . . . . . . . . . . . . 10

5.7 Subcritical free shear layer time-averaged streamwise vorticity flood plots in the $x=0.35 \mathrm{~L}$ plane. . . . . . . . . . . 103

5.8 Spanwise wavenumber spectra for the subcritical shear layer at $\mathbf{y} / \theta_{\mathbf{f}}=0.3 \ldots \ldots \ldots \ldots \ldots \ldots \ldots \ldots$

5.9 Instantaneous isocontours of spanwise and streamwise vorticity for one K-H vortex-shedding cycle for the supercritical shear layer (green: spanwise vorticity $\left(\omega_{z}=20 \mathrm{~s}^{-1}\right)$; red and blue: streamwise vorticity $\left.\left(\omega_{\mathbf{x}}= \pm \mathbf{2 0 \mathbf { s } ^ { - 1 }}\right)\right) \ldots \ldots \ldots \ldots$

5.10 Development of streamwise curvature in the free shear layer by $\mathrm{K}-\mathrm{H}$ instability. . . . . . . . . . . . . . . . . . . 108 
5.11 An example of the formation of $\mathrm{K}-\mathrm{H}$ and Holmboe vortices in one cycle of $\mathrm{K}-\mathrm{H}$ vortex-shedding. . . . . . . . . . . . 109

5.12 Spanwise wavenumber spectra for the supercritical shear layer

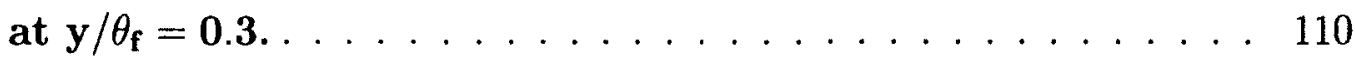

A.1 Comparison of different fluids near the critical point(IAPWS,

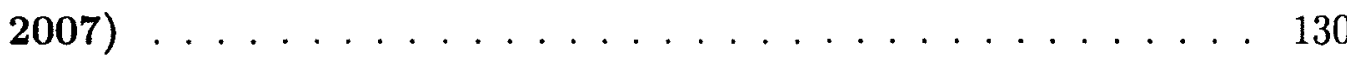

A.2 Comparison of enthalpy vs pressure and temperature using the IAPWS-95 and IAPWS-97 formulations . . . . . . . . 133

A.3 Comparison of density vs pressure and enthalpy for NIST using the IAPWS-95 and IAPWS-97 formulations . . . . . . 134

A.4 Comparison of density vs pressure and temperature using the IAPWS-95 and IAPWS-97 formulations . . . . . . . . . 134

A.5 Comparison of specific isobaric heat capacity vs pressure and enthalpy using the IAPWS-95 and IAPWS-97 formulations . 135

A.6 Comparison of specific isobaric heat capacity vs pressure and temperature using the IAPWS-95 and IAPWS-97 formulations 135

A.7 Comparison of viscosity vs pressure and enthalpy using the

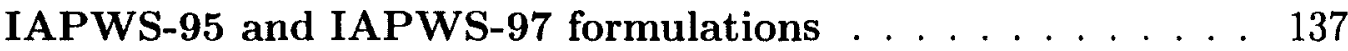

A.8 Comparison of viscosity vs pressure and temperature using the IAPWS-95 and IAPWS-97 formulations . . . . . . . . . 137

A.9 Comparison of thermal conductivity contours vs pressure and enthalpy using the IAPWS-95 and IAPWS-97 formulations . 138

A.10 Comparison of thermal conductivity contours vs pressure and temperature using the IAPWS-95 and IAPWS-97 formulations 138

A.11 Uncertainty of IAPWS-95 formulation compared to experimental data (Wagner and Prub, 2002) $\ldots \ldots \ldots$ 
A.12 Variation of $c_{p}$ against temperature at $P=24 \mathrm{MPa}$. Red $c_{p}$. Green: Non dimensional $c_{p}$ variation $\frac{\partial \mathrm{C}_{\mathrm{p}}}{\partial \mathrm{T}} \ldots \ldots \ldots . \ldots 142$

A.13 Variation of $c_{p}$ against pressure at $T=647 \mathrm{~K}$. Red $c_{p}$. Green Non dimensional $c_{p}$ variation $\frac{\partial \mathrm{C}_{\mathrm{p}}}{\partial \mathrm{P}} \quad \ldots \ldots \ldots . \ldots 142$

A.14 Wall-normal variation in (a) density, (b) dynamic viscosity, (c) temperature, and (d) velocity magnitude at $x / L=0.9$ and $\mathrm{z} / \mathrm{L}=0.5$ for simulations with fine, medium, and coarse fluidproperty increment sizes. Dashed lines show relative error for

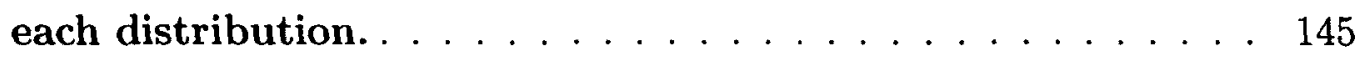

B.1 Comparison of time-mean and perturbation velocity profiles in the $\mathbf{R e}_{\theta}=320, \mathbf{R}=1.88$ test cases at two wall proximities. 154 


\title{
Nomenclature
}

\author{
$a_{c}$ \\ Centripetal acceleration $\left(\mathrm{m} / \mathrm{s}^{2}\right)$ \\ $a_{i}$ \\ Left-hand-side coefficient for linear system of equations \\ $a_{p}$ \\ Normalization parameter for the residual that is proportional to the \\ size of the computational domain \\ $A_{0}$ \\ $[A]$ \\ $b_{i}$ \\ Perturbation amplitude \\ Initial perturbation amplitude \\ Left-hand-side linear system coefficient matrix \\ Right-hand-side coefficients for linear system of equations \\ [b] \\ Right-hand-side vector of coefficients \\ c \\ Perturbation phase speed $(\mathrm{m} / \mathrm{s})$ \\ $c_{p}$ \\ Isobaric specific heat capacity $(k J / k g K)$ \\ $f$ \\ Frequency $(\mathrm{Hz})$ \\ F \\ Non-dimensional frequency, $F=\omega \nu / U_{f}^{2}$ \\ $g$ \\ Gravitational acceleration $\left(\mathrm{m} / \mathrm{s}^{2}\right)$ \\ $h$ \\ Enthalpy $(k J / k g)$ \\ $h_{0}$ \\ Total enthalpy $(k J / k g)$ \\ $H$ \\ Boundary layer shape factor \\ $J$ \\ Flow Richardson number (see Equation 2.10) \\ $k$ \\ Thermal conductivity $\left(W / m^{2} K\right)$
}


$\dot{m}$

$N$

$N_{i}$

$\Delta n_{i}, \Delta n_{j}$

$P$

$P_{0}$

$\operatorname{Pr}$

$Q$

$R$

$\bar{R}$

$r$

$r_{0}$

$(r, s, t)$

$R e_{D}$

$R e_{x, s-t r}$

$R e_{y, \text { in }}$

$R e_{\delta s^{*}}$

$R e_{\theta s}$
Streamwise length of the mixing region downstream of the splitterplate trailing edge $(\mathrm{mm})$

Mass flow rate $(\mathrm{kg} / \mathrm{s})$

Buoyant frequency, $N=\sqrt{(g / \rho)(-d \rho / d y)}$ (Lawrence et. al., 1991)

Finite volume shape function

Discrete surface normal vector in the $i=1,2,3$ or $j=1,2,3$ direction in tensor notation $\left(\mathrm{m}^{2}\right)$

Pressure $(\mathrm{Pa})$

Total pressure $(\mathrm{Pa})$

Fluid Prandtl number, $\operatorname{Pr}=c_{p} \mu / k$

Second invariant of the velocity gradient tensor $\left(1 / s^{2}\right)$

Velocity difference ratio, $R=2\left(U_{1}-U_{2}\right) /\left(U_{1}+U_{2}\right)$

Ideal gas constant $(\mathrm{J} / \mathrm{kgK})$

Radius $(m)$

Radius of Rankine vortex boundary $(m)$

Parametric co-ordinates on the surface of a finite element element $(m)$

Reynolds number based on pipe diameter, $R e_{D}=U_{1} D / \nu$

Reynolds number to transition onset, $R e_{s-t r}=U_{1} x_{s-t r} / \nu$

Modified Reynolds number based on the wall-normal position of in-

flection point (Diwan and Ramesh, 2009; see Equation 2.8)

Reynolds number based on displacement thickness of the boundary layer in the high-speed stream at the splitter-plate trailing edge, $R_{\delta s^{*}}=U_{1} \delta_{s}^{*} / \nu$

Reynolds number based on momentum thickness of the boundary layer in the high-speed stream at the splitter-plate trailing edge, $R_{\theta s}=U_{1} \theta_{s} / \nu$ 


\begin{tabular}{|c|c|}
\hline$R e_{\theta f}$ & $\begin{array}{l}\text { Reynolds number based on momentum thickness of the free-shear } \\
\text { layer in the high-speed stream at the splitter-plate trailing edge, } \\
R_{\theta f}=U_{1} \theta_{f} / \nu\end{array}$ \\
\hline$\left[r_{\phi}\right]$ & Residual matrix \\
\hline$\sigma$ & Standard deviation of a quantity \\
\hline$S E$ & Standard error of a quantity \\
\hline$S t_{\theta f}$ & $\begin{array}{l}\text { Strouhal number based on free-shear layer momentum thickness and } \\
\text { spanwise mean velocity, } S t_{\theta s}=f \theta_{f} / \bar{U}\end{array}$ \\
\hline$S t_{\theta s}$ & $\begin{array}{l}\text { Strouhal number based on momentum thickness and freestream ve- } \\
\text { locity in the high-speed stream at the splitter-plate trailing edge, } \\
S t_{\theta s}=f \theta_{s} / U_{1}\end{array}$ \\
\hline$T$ & Temperature $(K)$ \\
\hline$T_{m}$ & Spanwise-averaged temperature, $T_{m}=\left(T_{1}+T_{2}\right) / 2(K)$ \\
\hline $\mathscr{T}$ & Vortex shedding period $(s)$ \\
\hline$t$ & Time $(s)$ \\
\hline$\Delta t$ & Timestep $(s)$ \\
\hline$u_{\tau}$ & Friction velocity, $u_{\tau}=\left[\left.\nu(d U / d y)\right|_{w}\right]^{1 / 2}$ \\
\hline$(u, v, w)$ & Perturbation-velocity mode shape in the $\mathrm{x}, \mathrm{y}$ and $\mathrm{z}$ directions $(\mathrm{m} / \mathrm{s})$ \\
\hline$(\hat{u}, \hat{v}, \hat{w})$ & Instantaneous perturbation velocity fluctuation components $(\mathrm{m} / \mathrm{s})$ \\
\hline$\widetilde{U}$ & Spanwise-averaged streamwise velocity, $\bar{U}=\left(U_{1}+U_{2}\right) / 2(\mathrm{~m} / \mathrm{s})$ \\
\hline$U_{f}$ & Freestream velocity magnitude $(\mathrm{m} / \mathrm{s})$ \\
\hline$U_{i}, U_{j}$ & $\begin{array}{l}\text { Cartesian velocity component in the } i=1,2,3 \text { or } j=1,2,3 \text { direction } \\
\text { in tensor notation }(\mathrm{m} / \mathrm{s})\end{array}$ \\
\hline$(U, V, W)$ & Time-averaged velocity components in the $\mathrm{x}, \mathrm{y}$ and $\mathrm{z}$ directions $(\mathrm{m} / \mathrm{s})$ \\
\hline V & Velocity vector $(\mathrm{m} / \mathrm{s})$ \\
\hline $\mathscr{V}$ & Volume of element $\left(\mathrm{m}^{3}\right)$ \\
\hline
\end{tabular}




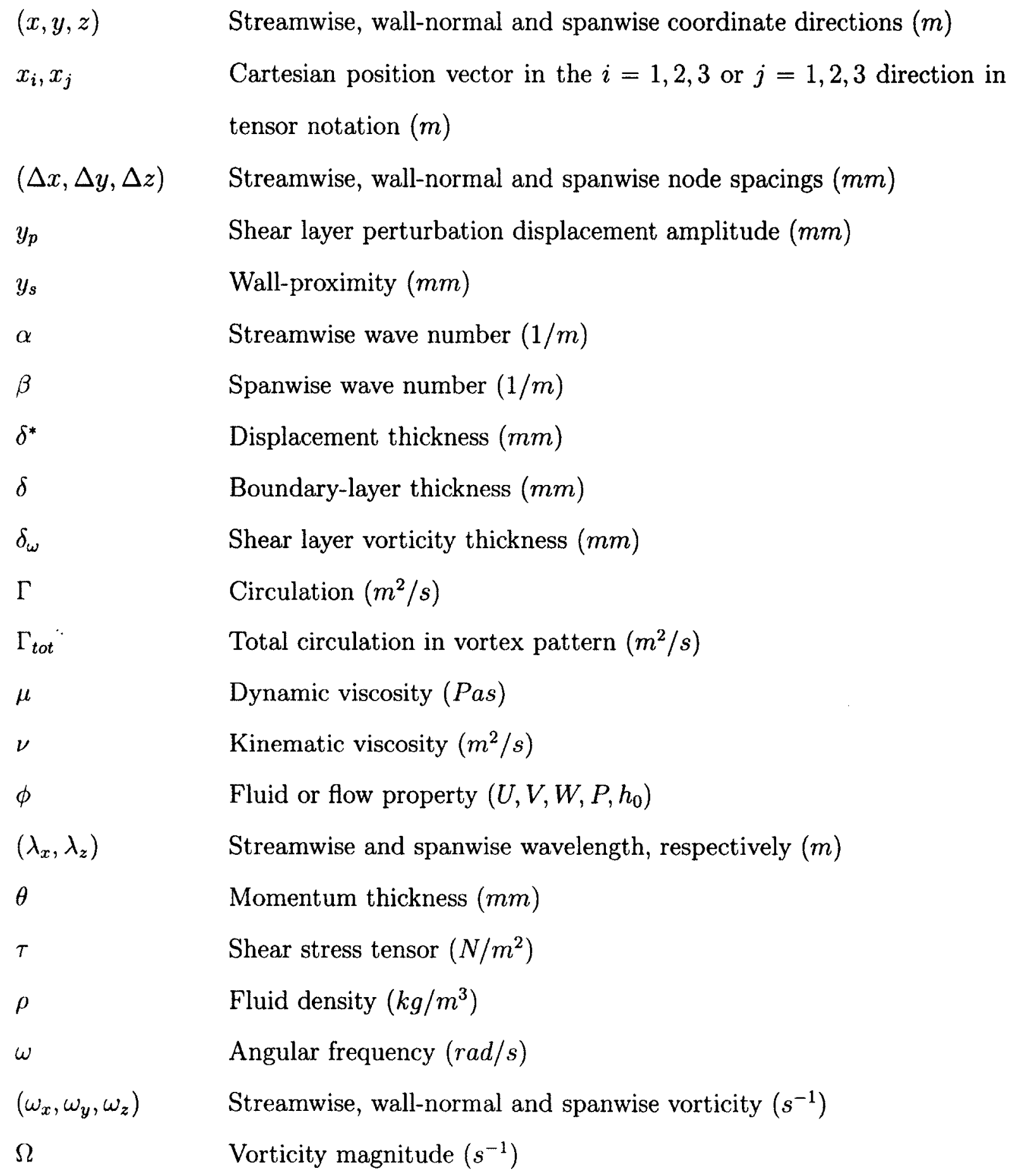

\section{Overbars}

Spanwise-averaged value of a parameter 


\section{Subscripts}

1

2

base

$c$

$f$

$i$

image

induced

in

ip

main

MA

$\max$

$n$

$p c$

$r$

ref

rms

$s$

s-tr
High-momentum stream

Low-momentum stream

Baseflow quantity

Parameter is evaluated at the thermodynamic critical point

Parameter is evaluated at streamwise location of fully-developed shear-

layer velocity profile

Imaginary component of a complex number

Image vortex

Induced quantity

Parameter is evaluated at the inflection point

Integration point

Main vortex

Most-amplified instability

Local maximum quantity

Natural frequency

Parameter is evaluated at the thermodynamic pseudocritical point at $24 \mathrm{MPa}$

Real component of a complex number

Parameter is evaluated at a reference value

Root mean square

Parameter is evaluated at the high-momentum stream at the splitterplate trailing edge

From the splitter plate trailing edge to site of transition onset 


\section{Superscripts}

1

*

$n+1$

\section{Acronyms}

BL

FS

DNS

Ho

K-H

LST

PSD

T-S
Derivatives with respective to wall-normal $(y)$ velocity

Normalized parameter

Quantity expressed in wall units based on friction velocity and fluid-

property values in the high-speed stream at the splitter-plate trailing

edge

Present iteration

Next iteration
Boundary layer

Free shear layer .

Direct numerical simulation

Holmboe

Kelvin-Helmholtz

Linear stability theory

Power spectral density

Tollmien-Schlichting 


\section{Chapter 1}

\section{Introduction}

\subsection{Motivation}

Free shear layers are created when two flows of differing velocities meet at an interface. These shear layers are observed in numerous engineering applications including fuel injection in combustors, chemical reaction layers, separated flows on airfoils, wakes behind wings and in several atmospheric and astronomical phenomena (Ho and Huerre, 1984). Free shear layers promote fluid mixing because of their inherent instability dictated by the presence of an inflectional velocity profile. Two applications of free shear layers that prompted this study are related to the aerodynamic optimization of turbine blades in gas turbine engines and the use of supercritical water for the cooling of next-generation nuclear reactor fuel bundles. These seemingly disconnected applications of shear layers are strongly affected by the same modes of instability. However, the details of flow development under the influence of instability modes differ due to variations in properties of the flow field including the geometry of the free shear layer, the hydrodynamic properties of the flow and the thermophysical properties of the fluid. The focus of this thesis is to comparatively study the effects of two such factors on the stability of free shear layers.

Manufacturers of low-pressure turbines for gas-turbine engines are moving toward 
higher aerodynamic blade loading to meet the demand for lighter engines (Curtis et al., 1997). Low-pressure turbines often operate at low Reynolds numbers where the laminar boundary layer on the blade suction-surface is prone to separation. The separated flow tends to quickly transition into turbulence and, due to the enhanced wall-normal mixing in the turbulent state, reattachment of the shear layer to the wall is promoted. Although the presence of such flow configurations, referred to as "separation bubbles" in the literature, reduces the aerodynamic efficiency of blades, reattachment of the separated flow is more favourable than a fully-stalled blade (Mayle, 1991; Walker, 1993). Figure 1.1a shows an example of such a separation bubble on an airfoil.

In recent years, the author's research group has been extensively involved in the measurement, numerical simulation and semi-analytical modelling of blade-surface boundary layers with separation bubbles (e.g. Roberts and Yaras, 2005 a,b; McAuliffe and Yaras, 2007 a,b; Brinkerhoff and Yaras, 2011). Despite such substantial research efforts by the author's research group and others, there still remain outstanding questions on the physics of separation-bubble flows. One of these outstanding questions is the large spread in non-dimensional frequency of the most amplified instability associated with the formation of a separation bubble. It is speculated that free shear layers near walls, such as the separated shear layer of a separation bubble, have instability characteristics that are modified by the proximity of the shear layer to the wall (e.g. Rist and Maucher, 2002). The present study aims to contribute to our understanding of the effects of wall proximity on the development of the primary instability mode in free shear layers by investigating a simpli

ed flow con

gratin as shown on $1.1 \mathrm{~b}$.

Another factor affecting the stability of free shear layers is the presence of large spatial gradients in thermophysical properties across the shear layer that occur when 


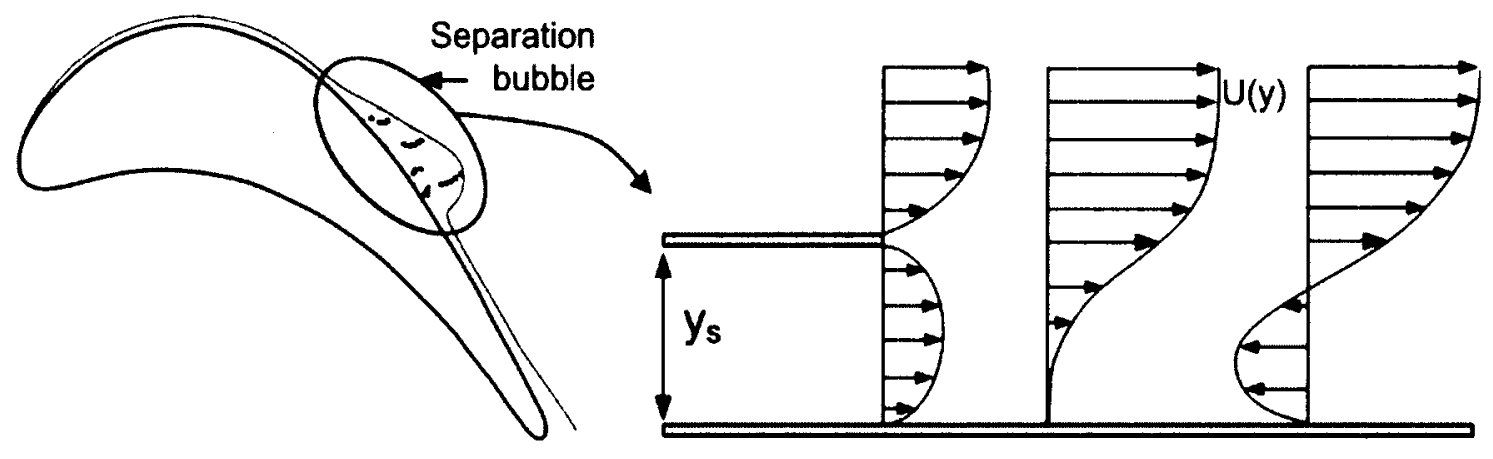

(a)

(b)

Figure 1.1: (a) Diagram of a laminar separation bubble on a turbine blade (adapted from Brinkerhoff, 2012). (b) Simplified flow geometry used to investigate the effect of wall proximity on the stability of a shear layer.

the working fluid is in a supercritical state. The Generation IV International Forum (GIF) is investigating the use of supercritical water as the working fluid for cooling of nuclear fuel bundles to take advantage of increased thermal efficiency at higher heat source temperatures without the complicating aspects of heat transfer involving multi-phase fluids (Pioro et al., 2003). However, there is evidence that under certain heat and mass flux conditions with the working fluid in supercritical thermodynamic state, the convection heat transfer rate is lower than the expected levels (Yamagata et al., 1971). A thorough understanding of the flow and heat transfer physics responsible for such heat-transfer variations is essential for the design of reliable nuclear-reactor cooling systems utilizing supercritical water. In collaboration with Atomic Energy Canada Ltd., the author's research group is undertaking a series of experimental and numerical studies to provide insight into this heat transfer problem. The research program is designed to develop this insight through the study of a series of idealized configurations in which the effects of geometric, thermal and flow parameters on the heat transfer process are studied systematically. The present study contributes to this effort through an investigation of the instability development in a laminar free 


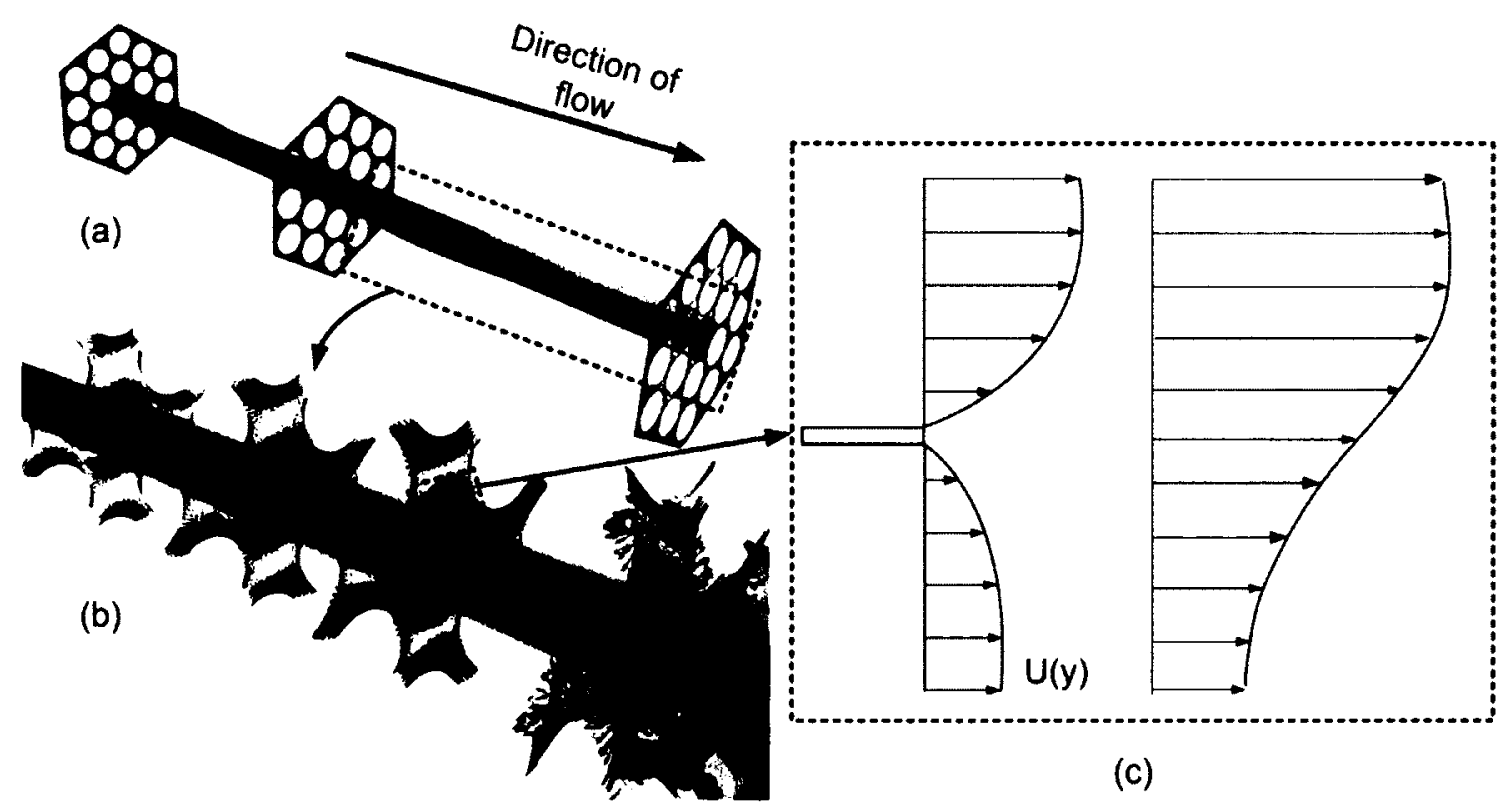

Figure 1.2: (a) Nuclear fuel rod in a fuel bundle (adapted from Hansen and Owen, 2008). (b) Shear layer development (shown in green/yellow) in flow through fuel bundle (adapted from Yan and Karoutas, 2011). (c) Simplified flow geometry used to investigate the effect of cross-stream property variations on shear layers composed of supercritical fluid.

shear layer when the working fluid is supercritical water. The temperature gradient imposed across such a shear layer produces thermophysical property gradients that are comparable to those that would be observed in the flow field within a nuclear fuelrod bundle cooled by supercritical water. Figure 1.2a illustrates an example of a fuel rod as a part of a nuclear fuel bundle that is representative of the fuel bundles in such supercritical water-cooled reactors. Shear layer configurations may develop on the fuel rod due to geometric features such as split spacers and wire-wrap spacers, as shown by the computed flow visualization by Jan and Karoutas (2012) in Figure 1.2b. Studying simplified shear layers, such as those shown in Figure 1.2c, in this context enables a fundamental investigation of the effects of supercritical thermodynamic state on the instability of a shear layer without the complicating effects of wall proximity, flow turbulence and other flow features typical of a base flow in a fuel-bundle geometry. 


\subsection{Objectives}

The first part of this thesis investigates the role of proximity to a wall in modifying the site of transition onset, the natural frequency of vortex shedding, and the development of the vortical structures in a laminar free shear-layer. The study is performed at two Reynolds numbers and velocity ratios to establish sensitivity of the flow development to these two parameters. The second part of this thesis aims to establish the dynamics of flow instabilities in a free shear layer with cross-stream thermophysical property gradients that are comparable to those that would develop in supercritical water cooling the fuel rods in a nuclear fuel bundle.

\subsection{Approach}

The study of free shear layer development in close proximity of a wall is based primarily on the time-dependent numerical solution of the Navier-Stokes equations in a two-dimensional domain using the ANSYS CFX commercial software package. Limiting the spatial domain allows for computational efficiency and thus a relatively large test matrix of Reynolds numbers, velocity ratios and wall proximities. At the same time, this choice constrains the study to the early part of free shear layer development, up to the streamwise location where flow instabilities would be expected to develop three-dimensional features. Despite this constraint, study of the shear layer during its two-dimensional early development phase is expected to provide the answers sought in this thesis. The Navier-Stokes computations are complemented by linear stability analyses and potential flow computations. These mathematically simpler analyses serve to guide the interpretation of the flow computed through the Navier-Stokes simulations.

For the study of the effects of thermophysical property gradients on laminar free 
shear layer development in the context of supercritical fluids, the direct numerical simulation (DNS) approach is taken. The fine spatial and temporal resolution of this approach allows for natural development of all flow instabilities and resultant turbulent flow structures without any assumptions regarding these flow phenomena. The ANSYS CFX software package is used for these computations. Suitability of this software for accurate DNS computations has been established by the author's research group through extensive validation efforts (e.g. Azih et al. 2011, 2012). 


\section{Chapter 2}

\section{Literature Survey}

This chapter reviews the published literature on shear layers in close proximity to walls and shear layers with cross-stream thermophysical property gradients. Section 2.1 gives an overview of the role of attached and separated shear layers in the aerodynamic optimization of gas turbine engines. Section 2.2 discusses the instability of attached laminar boundary layers, and Section 2.3 discusses the instability of shear layers, particularly planar free shear layers and separated shear layers, with emphasis on the effects of wall proximity and cross-stream property gradients on the stability of the shear layer. Finally, Section 2.4 reviews the literature on the hydrodynamic stability of fluids in a supercritical thermodynamic state to provide a context for part of the work presented in this thesis.

\subsection{Separation Bubbles on Axial Turbine Blades}

Modern trends in the design of low-pressure axial turbine blades for gas-turbine engines are moving towards increased aerodynamic loading in an attempt to lower the weight and cost of the engine (e.g. Hourmouziadis, 1989; Curtis et al., 1997). Increased aerodynamic loading implies stronger adverse pressure gradients on the suction surface of the blade, thereby increasing the likelihood that the attached laminar 
boundary layer may separate from the blade surface before transitioning to a turbulent state, increasing the drag and decreasing the efficiency of the blade (Mayle, 1991). Rapid amplification of disturbances in the separated shear layer promotes laminar-to-turbulent transition which in turn promotes reattachment due to the enhanced momentum exchange arising from turbulence (Mayle, 1991; Walker, 1993). This sequence of separation, transition, and turbulent reattachment forms a "separation bubble" when viewed in a time-averaged sense.

The anatomy of a separation bubble is shown in Figure 2.1. This diagram shows that the attached boundary layer (Region I) separates from the blade surface due to the adverse streamwise pressure gradient, forming a separated shear layer with an inflectional velocity profile (Region II) (Horton, 1968; Alam and Sandham, 2000). Separation of the shear layer creates a region of constant pressure in the streamwise (x) direction (Mayle, 1991; Walker, 1993). The region below the separated shear layer (Region III) was originally thought to be a zone of stagnant dead air, however, several studies (e.g. McAuliffe, 2007 a,b; Dovgal et al., 1994) have shown that there is may be reversed flow of significant magnitude within the separation bubble. Following the turbulent reattachment (Region IV) of the separated shear layer, the downstream flow is characterized by a region of pressure recovery (Mayle, 1991).

Due to the impact of the separation bubble on the blade performance, accurate prediction of the transition-onset location and the rate of transition is important for designers of gas turbine engines, and refined engineering models have been developed for the purpose of predicting these quantities for a range of Reynolds numbers, pressure gradients, and freestream conditions (e.g. Roberts and Yaras, 2005). Further improvement of such models requires a clear understanding of the instability mechanisms that contribute to transition in separated flows. Due to the presence of attached laminar flow upstream of separation, instability and transition in separated shear layers on gas-turbine blades likely resemble the instability characteristics of both 


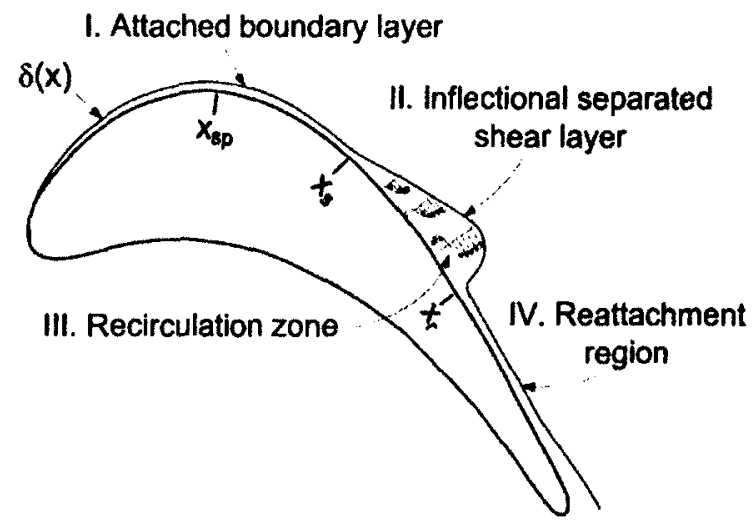

Figure 2.1: Schematic of a laminar separation bubble (Brinkerhoff, 2012).

attached and free shear layers (Alam and Sandham, 2000). Therefore, Sections 2.2 and 2.3 will respectively review the literature on the development of stability characteristics in attached boundary layers and separated shear layers leading to breakdown to turbulence. While the freestream flow in low-pressure turbines of gas-turbine engines is typically of high turbulence level, in keeping with the objective of studying flow instability in an isolated setting to pinpoint certain cause-and-effect relationships, the present study and thus the accompanying literature survey is limited to the conditions of low freestream turbulence.

\subsection{Attached Boundary Layer Stability}

Boundary layers are formed on a solid surface as the no-slip condition at the wall creates viscous stresses that alter the velocity profile of the flow in the vicinity of the wall (White, 1991). Breakdown of a laminar boundary layer into turbulence depends on environmental disturbance levels (Morkovin, 1969). Sections 2.2.1, 2.2.2 and 2.2.3 discuss the primary instability, secondary instability and breakdown mechanisms in "natural transition", that is transition in low disturbance environments. 


\subsubsection{Primary Instability Mechanism}

The primary instability mode is the first instability to appear in a boundary layer. To theoretically examine the onset of instability in a laminar boundary layer, Orr (1907b) and Sommerfeld (1908) applied a perturbation of the form given in Equation 2.1 to a laminar, parallel base-flow:

$$
\hat{v}=v(y) e^{i \alpha(x+c t)}
$$

Where $\hat{v}$ is the instantaneous wall-normal perturbation velocity, $v(y)$ is the perturbation mode shape, $c$ is the perturbation phase velocity, $\alpha$ is the streamwise wavenumber such that perturbation frequency is $\omega=\alpha c$. The incompressible NavierStokes equations, which describe the conservation of mass and momentum in fluid flow, are written in the perturbation form by decomposing the velocity into a steady and fluctuating component. By using the perturbation velocity expression given in Equation 2.1 for the fluctuating components of velocity, through a series of straight forward mathematical steps the Orr-Sommerfeld equation is obtained.

$$
(U-c)\left(v^{\prime \prime}-\alpha^{2} v\right)-U^{\prime \prime} v+\frac{i \nu}{\alpha}\left(v^{\prime \prime \prime \prime}-2 \alpha^{2} v^{\prime \prime}+\alpha^{4} v\right)=0
$$

Here $U$ is the baseflow streamwise velocity, $\nu$ is the kinematic viscosity of the fluid and primes denote differentiation in the wall-normal $(\mathrm{y})$ direction. The characteristics of this ordinary differential equation describe the stability of plane parallel flows. The Orr-Sommerfeld equation may be re-written using non-dimensional quantities thus elucidating the role of Reynolds number in the stability characteristics of a laminar parallel flow:

$$
\underbrace{\left(U^{*}-c^{*}\right)\left(v^{* \prime \prime}-\alpha_{\delta^{*}}^{2} v^{*}\right)-U^{* \prime \prime} v^{*}}_{\text {Inviscid Terms }}+\underbrace{\frac{i}{\alpha_{\delta^{*}} \operatorname{Re}_{\delta^{*}}}\left(v^{* \prime \prime \prime \prime}-2 \alpha_{\delta^{*}}^{2} v^{* \prime \prime}+\alpha_{\delta^{*}}^{4} v^{*}\right)}_{\text {Viscous Terms }}=0
$$


where,

$$
v^{*}=\frac{v}{U_{f}}, \quad U^{*}=\frac{U}{U_{f}}, \quad c^{*}=\frac{c}{U_{f}}, \quad \alpha_{\delta^{*}}=\alpha \delta^{*}, \quad R e_{\delta^{*}}=\frac{U_{f} \delta^{*}}{\nu}
$$

Here, $U_{f}$ is the freestream velocity and $\delta^{*}$ is the displacement thickness of the shear layer as determined by its velocity profile. Equation 2.3 reveals that the properties that affect the stability of the laminar shear layer include the shape of the baseflow velocity profile, the flow Reynolds number and the properties of the disturbance including its phase-speed and streamwise wave-number.

This linear stability theory can be used to find the critical Reynolds number corresponding to the streamwise location of the start of instability (Schlichting, 1968). The unstable perturbation waves that amplify in a laminar boundary layer are commonly referred to as Tollmien-Schlichting (T-S) waves. The first recognition of the instability of the zero-streamwise-pressure gradient (Blasius) boundary layer profile was made by Tollmien (1929) and the provision of an analytical solution was made by Schlichting (1979). Analysis of the Orr-Sommerfeld equation shows that viscosity may have a destabilizing effect within the critical layer where $U=c$, causing velocity profiles that are stable in the absence of viscosity to become unstable. A detailed review of the critical-layer instability is presented by Mack (1984a) and Maslow (1977), amongst others.

Equation 2.3 shows that the primary role of the flow Reynolds number is to affect the relative influence of the viscous and inviscid terms. For low Reynolds numbers, the viscous instability modes become exacerbated. For large Reynolds numbers, the role of the fourth-order expression embedded in the Orr-Sommerfeld equation becomes insignificant and these terms may be neglected altogether in the inviscid limit. The spatial stability characteristics of the Orr-Sommerfeld equation are effectively shown on "thumb" diagrams as shown in Figure 2.2 where boundaries of neutral stability are drawn on a graph with the abscissa as the Reynolds number and the streamwise 


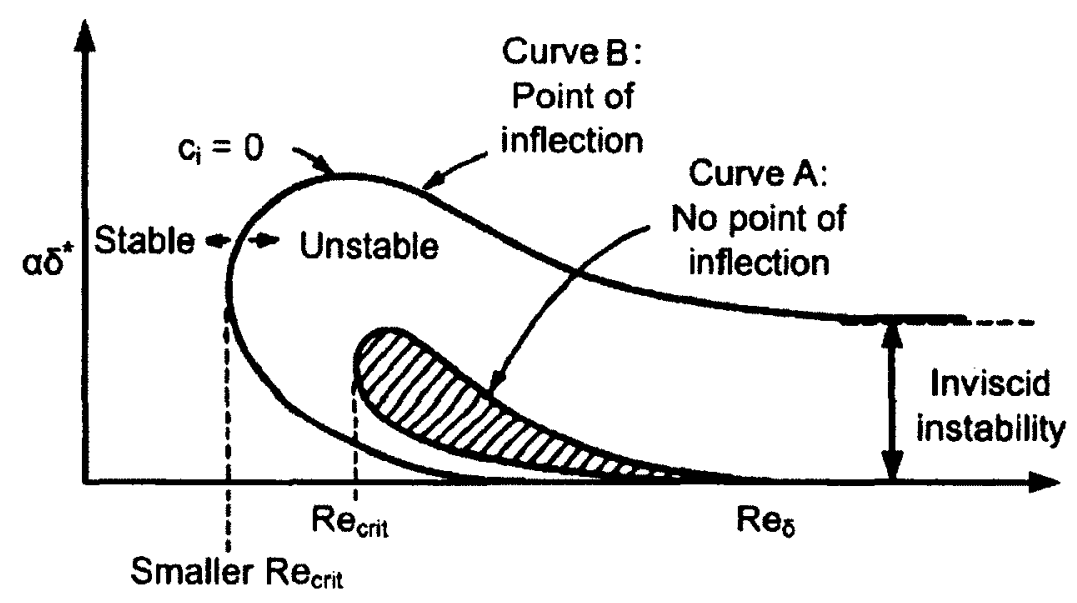

Figure 2.2: Schematic of stability "thumb plots" for velocity profiles with and without inflection points (White, 1991).

wave-number of the disturbance as the ordinate.

In addition to the Reynolds number and the wavenumber of the perturbation, the shape of the base-flow velocity profile is the third important factor affecting the stability of the flow field, and it is represented as contours of neutral stability superimposed on the thumb diagram. Figure 2.2 considers two typical neutral curves, one for a velocity profile, $U(y)$, that does not contain an inflection point (Curve A) and another that does contain an inflection point (Curve B). Curve $A$ shows that a velocity profile without an inflection point is unstable for a finite Reynolds number range starting at the critical Reynolds number. As the Reynolds number increases, the role of the viscous terms in Equation 2.3 reduces and the profile shows a reduction in the range of instability wavelengths until it becomes stable to all disturbances. Conversely, Curve B, which corresponds to a velocity profile with an inflection point, has a lower critical Reynolds number (Reed et al., 1996). At large Reynolds numbers the neutral stability curve does not close up, and the flow remains unstable over a certain disturbance wavelength even at infinitely large Reynolds numbers. At such high Reynolds numbers, an inviscid instability mechanism dominates. 


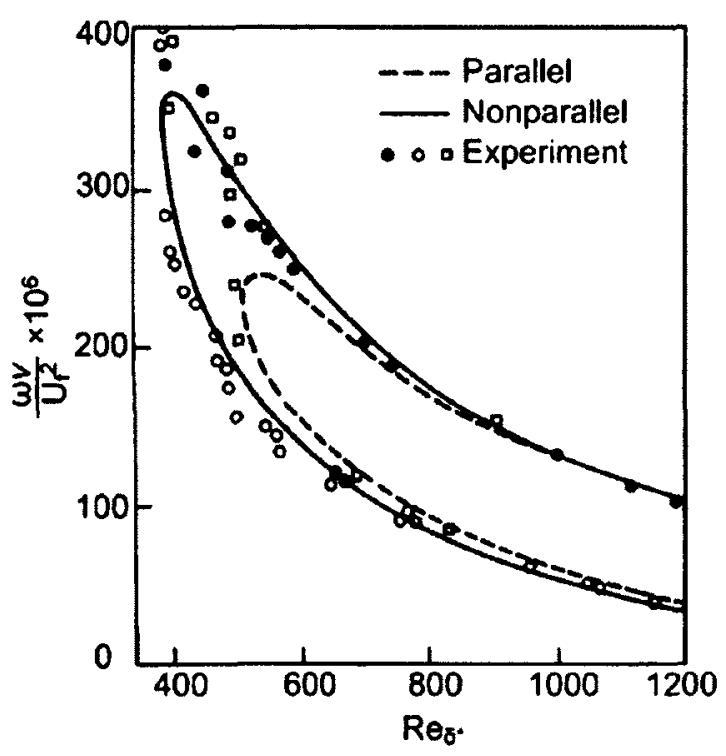

(a)

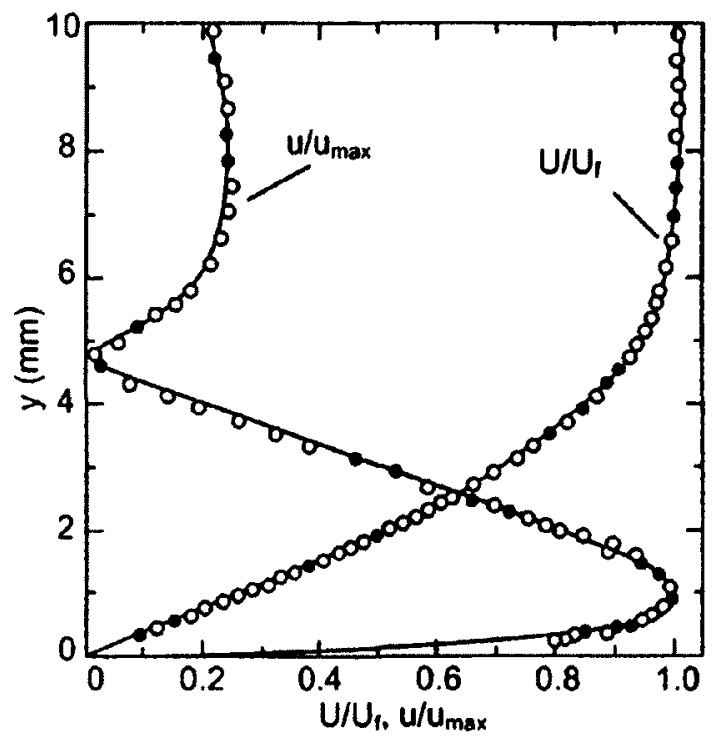

(b)

Figure 2.3: (a) Experimental measurements and theoretical prediction of T-S wave frequency (Schubauer and Skramstad, 1947). (b) Experimental (symbols) and theoretical (lines) mode shapes (Reed et al., 1996).

The Tollmien-Schlichting (T-S) instability was confirmed by the experimental studies of Schubauer and Skramstad (Schubauer and Skramstad, 1947). They not only showed that the T-S waves can be seen as fluctuations in the velocity components in oscilloscope time traces, but also demonstrated that the onset of instability corresponds to the eigenvalues and critical Reynolds number predicted by the linear stability theory (Schubauer and Skramstad, 1947). The T-S waves are characterized by a streamwise redistribution of vorticity around the critical layer where the viscous instability energy production primarily originates (Herbert, 1988). Their results, shown in Figure 2.3a, agree with the analytical solutions to the Orr-Sommerfeld equation provided by Tollmien and Schlichting (White, 1991).

Visualization of T-S instability often proves difficult. However, its presence is often captured in literature through plots of perturbation velocity profiles, which represent the eigenmodes of the disturbance, showing the amplification rate of the perturbation, 


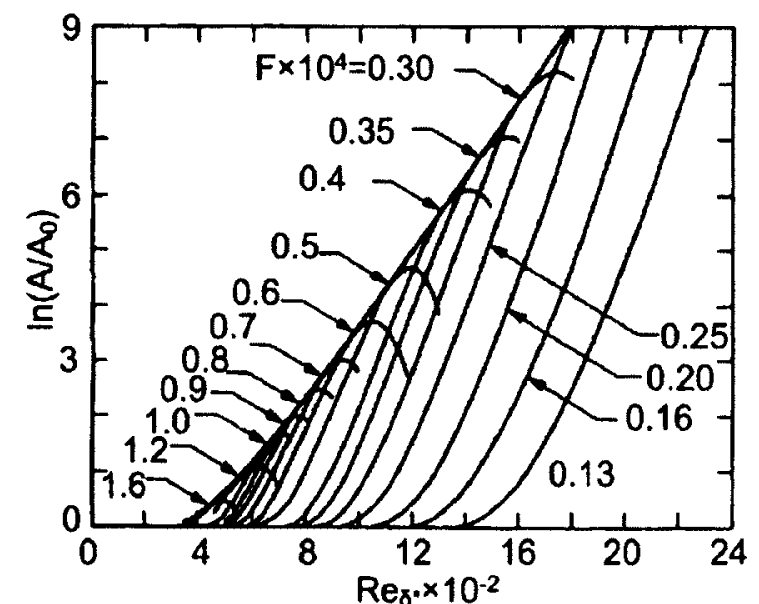

(a)

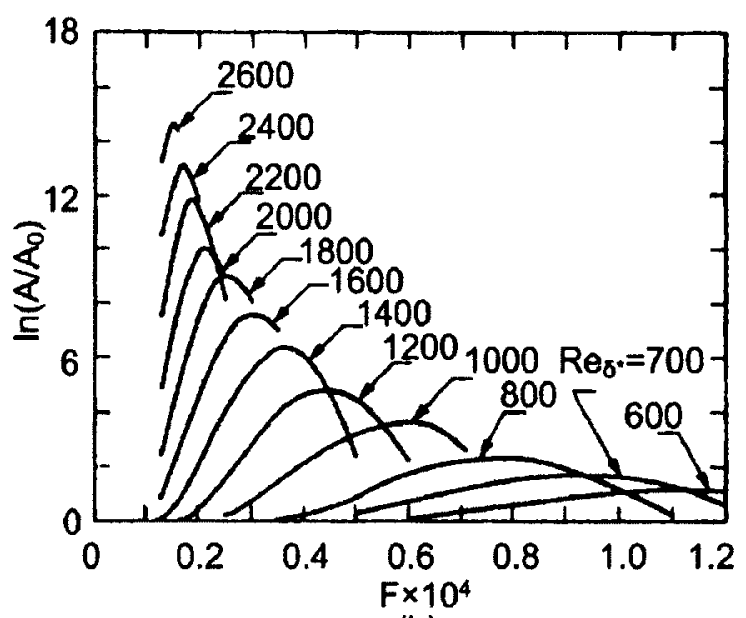

(b)

Figure 2.4: (a) Amplification rate and (b) frequency distribution of Tollmien-Schlichting waves for Blasius boundary layer as predicted by linear stability theory (adapted from Mack, 1984).

and power spectral density plots (Mack, 1984; Reynolds and Saric, 1986; Reed and Nayfeh, 1986). Analysis of the Orr-Sommerfeld equation by Reed and Nayfeh (1986) as well as the experimental study of Reynolds and Saric (1986) give convincing evidence that the primary mode of the T-S instability for a Blasius boundary layer has the form given in Figure 2.3b (Reed and Saric, 1996). The root-mean-square (rms) streamwise velocity fluctuation shows a double-humped trend where the perturbation amplitude rises from zero at the wall to a maximum at the critical layer, where the base-flow velocity equals the disturbance phase velocity, followed by an initial reduction, a secondary maximum and an ensuing reduction in fluctuation amplitude until the magnitude approaches the freestream value. This has been demonstrated to be a function of Reynolds number, with additional peaks being observed at higher Reynolds numbers (Nayfeh et al., 1988).

Mack (1984) presents the amplification rates and frequency-band distribution for the T-S waves for a Blasius boundary layer as shown in Figure 2.4, where $A$ represents the perturbation amplitude and $A_{0}$ is the initial amplitude. Here, $F=\omega \nu / U_{f}^{2}$ 
is a nondimensional frequency with $U_{f}$ being the freestream velocity and $\omega$ the angular frequency. Figure 2.4a shows the amplification rates as a function of Reynolds number for a range of instability frequencies. This diagram shows that below a critical Reynolds number, the growth rate of a perturbation is zero. Once the critical Reynolds number is reached, the T-S wave grows until it reaches a critical amplitude. Similarly, Figure 2.4b shows the frequency band of amplified waves for different Reynolds numbers. This plot shows that, as per the Orr-Sommerfield equation, low Reynolds numbers have shallow peaks that are distributed over a larger range of frequencies. However, higher Reynolds numbers are accompanied by a narrower band of highly amplified frequencies. Similar profiles are available for Falknar-Skan and other velocity profiles (Obremski et al., 1969; Wazzan et al., 1968). Walker et al. (1989) developed a correlation for the frequency of the most amplified T-S wave, denoted $f_{M A}$, based on the stability curves provided by Obremski et al. (1969) which can be expressed as:

$$
f_{M A}=\frac{3.2 U_{f}^{2}}{2 \pi \nu R e_{\delta^{*}}^{3 / 2}}
$$

where $f_{M A}$ is the frequency of the most-amplified perturbation, $R e_{\delta^{*}}$ is the flow Reynolds number based on the freestream velocity $U_{f}$ and the displacement thickness of the boundary layer.

\subsubsection{Secondary Instability Mechanism}

Following the primary T-S instability, a secondary instability develops in a laminar boundary layer as the T-S waves form waviness in the spanwise direction. This instability creates streaks (defined as spanwise variation in the streamwise velocity component) in the boundary layer that coincide with the presence of coherent vortical structures in the shape of the Greek letter $\Lambda$, shown in Figure 2.5 (Klebanoff and Tidstrom, 1972). Amongst others, Grek et al. (2000) assert that these $\Lambda$ structures are a 


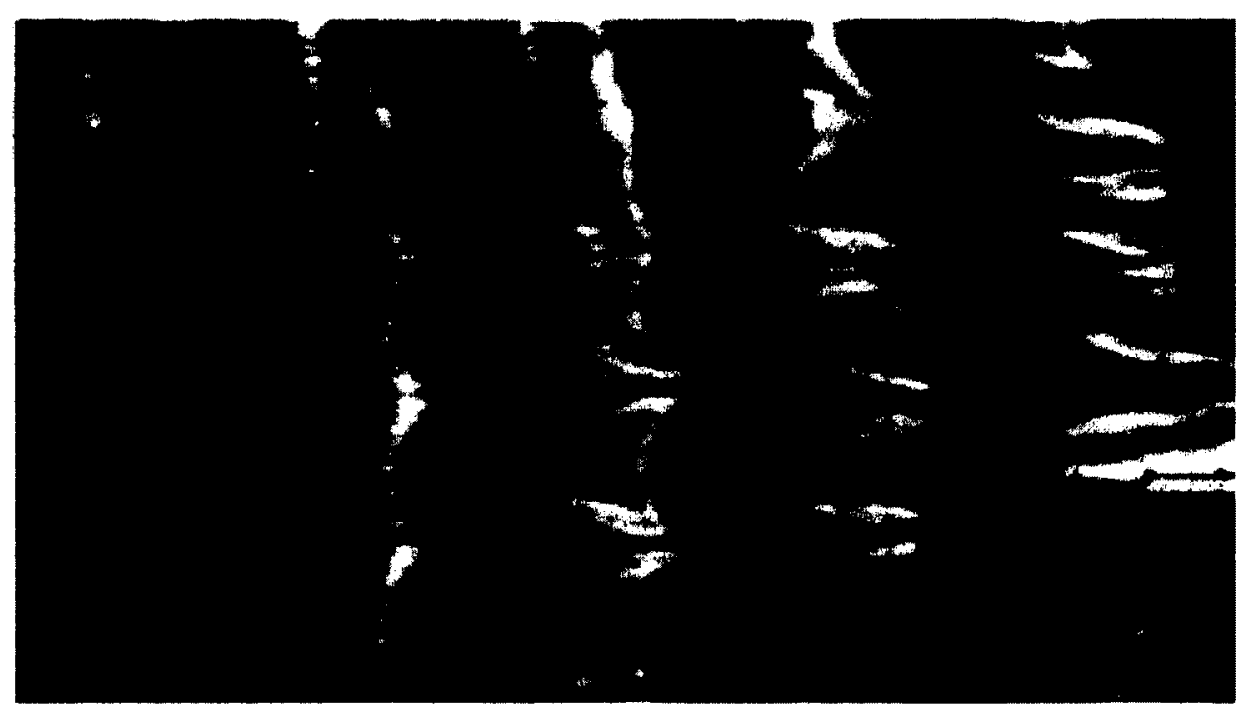

Figure 2.5: Aligned pattern of $\Lambda$-vortices (Herbert, 1988).

subset of a more general category of coherent structures known as "hairpin vortices", which are vortical structures commonly observed in fully turbulent boundary layers (Adrian et al., 2000; Smith et al., 1991). These $\Lambda$ vortices consists of a "head" and a "leg" segment that induce velocity in the surrounding boundary layer in the direction shown in Figure 2.6b (Smith et al., 1991). The self-induced wall-normal velocity on the head of the vortex lifts it to the high-momentum-stream portion of the boundary layer leading to the elongation of these vortices. Although there is often disagreement in the exact distinction between $\Lambda$ vortices and hairpin vortices, the elongated $\Lambda$ vortices are often referred to as "hairpin" vortices (Acarlar and Smith, 1987; Suponitsky et al., 2005). Smith et al. (1991) argue that these hairpin vortices grow laterally and form a spanwise array of streaks by the formation of secondary subsidiary vortices, as shown in Figure 2.6c. This model of a hairpin vortex explains many features of transitional and fully turbulent flow (Adrian et al., 2000). For example, the streaks observed by Klebanoff and Tidstrom (1972) in the streamwise velocity field may be explained as by the low-momentum fluid that is ejected upwards in the boundary 
(a)

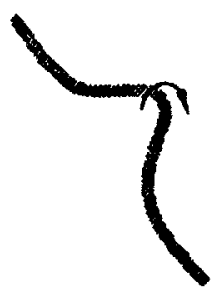

(b)

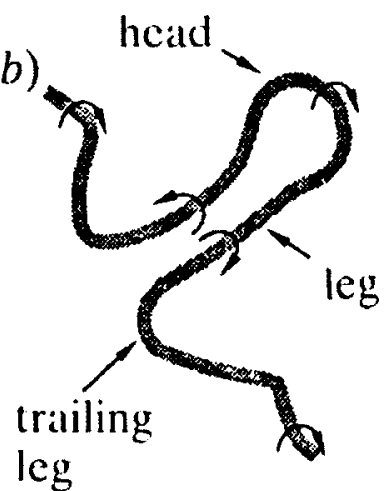

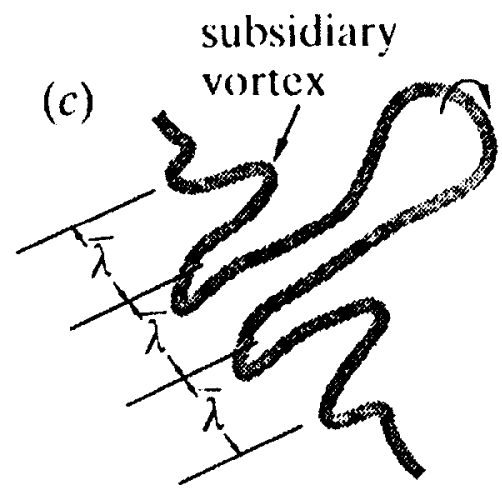

Figure 2.6: The development of a hairpin vortex (Smith et al., 1991).

layer between the legs of the hairpin vortices (Adrian et al., 2000).

More recently, an extension of linear stability theory, referred to as optimal perturbation theory, explains the formation of streamwise vortices by considering the spanwise perturbation that grows most-rapidly and robustly within parallel flows. Butler and Farrell (1993) demonstrated that the optimal perturbation theory predicts a spanwise wavelength in wall units of $\lambda_{z}^{+}=\lambda_{z} u_{\tau} / \nu=100$ for turbulent boundary layers, where $u_{\tau}$ is the friction velocity evaluated at the wall given by $u_{\tau}=\left[\left.\nu(d U / d y)\right|_{w}\right]^{1 / 2}$. Chernyshenko and Baig (2005) later proposed a model for spanwise instability development that predicts an optimal spanwise spacing as a function of normalized wall-normal position. They suggest that the physical mechanism of the spanwise instability development is through the development of wall-normal motion which advects high-momentum fluid towards the wall and low-momentum fluid away from the wall in alternating stripes. The spacing between consecutive streamwise vortices is governed by the balance of viscous diffusion and vanishing component of wall-normal velocity, the former strengthening as the streamwise vortices come closer together and the latter diminishing as the streamwise vortices move further apart (Chernyshenko and Baig, 2005). 


\subsubsection{Breakdown into Turbulence}

The formation of hairpin vortices commences the breakdown of an attached boundary layer through formation of turbulent spots. Smith et al. (1991) and Haidari and Smith (1994) show that from the primary hairpin vortex, secondary subsidiary vortices form around the kinks in the vorticity lines of the hairpin vortex, as shown in Figure 2.6. Singer and Joslin (1994) suggest that the rebounding of the hairpin vortices from the wall further contributes to spawning of vortices. As such, the spawning of subsequent generations of vortices from existing vortices drive the formation of turbulent spots (Smith et al., 1991; Singer and Joslin, 1994). Grek et al. (2000) demonstrated that hairpin vortices transform into turbulent spots by secondary high frequency disturbances produced at the legs of hairpin vortices. They describe transformation of these vortices into turbulent spots as accompanied by violent bursts.

Turbulent spots were first noticed by Emmons (1951) and since then numerous other researchers have observed these structures in transitioning flows. Figure 2.7a shows the top view of the streamwise growth and spreading of a turbulent spot using vorticity surfaces computed by Henningson et al. (1987) and Figure 2.7b shows the side view of a the turbulent spot. These turbulent spots have typically been observed to have an arrowhead shape pointing in the downstream direction (e.g. Wygnanski et al., 1976). Studies of turbulent spots by Wygnanski et al. (1976) and Cantwell et al. (1978) reveal an overhang region in the front of the turbulent spot that extends several boundary layer thicknesses above the spot centerline. Yaras (2007) provides a concise overview of the recent research on turbulent spot formation. Research by Schröder and Kompenhans (2004) and Yaras (2007) reveal a well-organized pattern of hairpin vortices and streaky structures within a turbulent spot. The growth of the turbulent spot through merging of neighbouring spots is responsible for the development of a fully-turbulent boundary layer. 


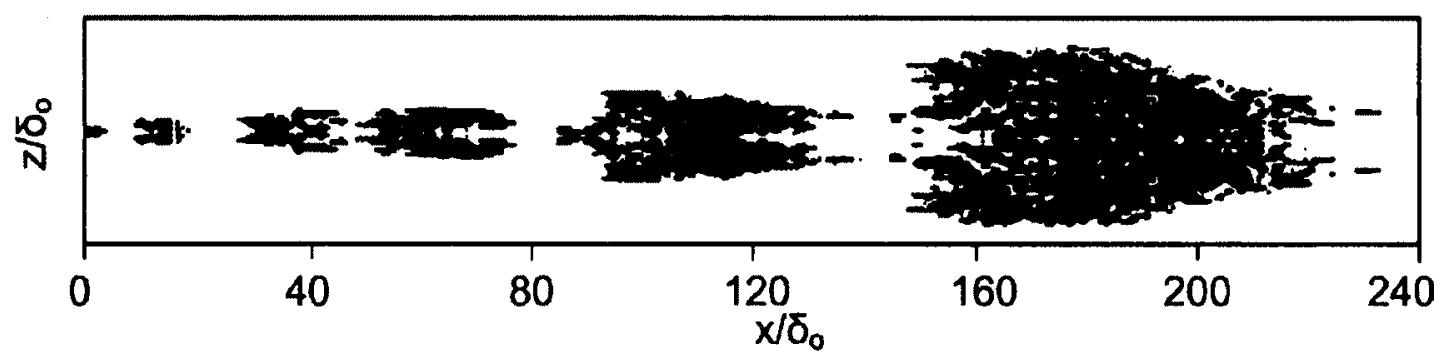

(a)

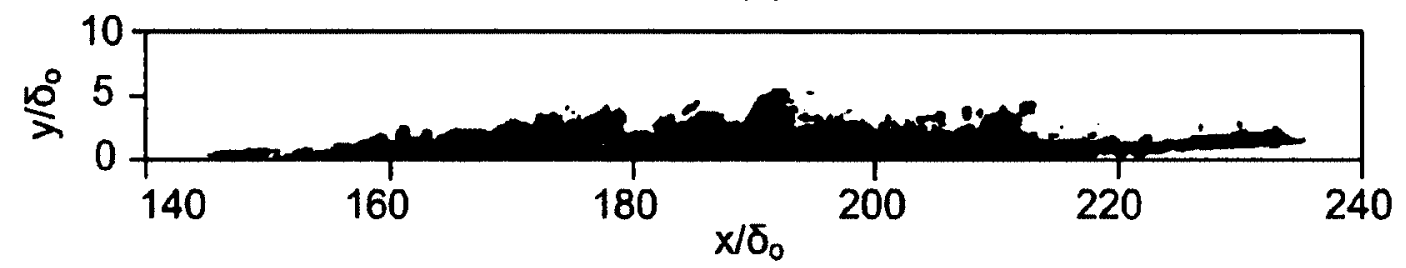

(b)

Figure 2.7: The structure of a turbulent spot (Henningson et al., 1987). Coordinate axes are normalized by initial boundary layer momentum thickness, $\delta_{\mathbf{o}}$.

\subsection{Free and Separated Shear Layer Stability}

In contrast to attached boundary layers, free and separated shear layers are formed either through the merging of two flows separated by a solid surface or by boundarylayer separation due to a geometric discontinuity or an adverse streamwise pressure gradient, as in the separation bubble shown in Figure 2.1. Free shear layers are typically studied far from solid surfaces, while separated shear layers typically occur in close proximity to a wall. Notwithstanding these differences, free shear layers and separated shear layers share numerous characteristics, as described in this section, and so they will hereafter be referred to collectively as simply free shear layers. The remaining sections of this chapter review the literature of the stability modes leading to laminar-to-turbulent transition in free shear layers, particularly addressing the effects of shear-layer proximity to a wall and cross-stream thermophysical property gradients. 


\subsubsection{Primary Instability Mechanism}

Similar to boundary layers, the early instability of free shear layers can be predicted using linear stability theory. However, the velocity profile in free shear layers has an inflection point causing the inviscid terms in Equation 2.3 to be significantly more pronounced than the viscous terms. Kelvin-Helmholtz (K-H) instability is the dominant inviscid instability mechanism for many flows with inflectional velocity profiles, including free shear layers and separated shear layers (Ho and Huerre, 1984; Dovgal et al., 1993). Therefore, in studies of the stability of free layers, the viscous terms are often neglected, producing the Rayleigh equation, shown in Equation 2.5.

$$
(U-c)\left(v^{\prime \prime}-\alpha^{2} v\right)-U^{\prime \prime} v=0
$$

Once again, $U$ is the baseflow velocity profile, $v(y)$ is the stream-normal velocity perturbation mode shape, $\alpha$ is the streamwise wavenumber, and the prime $\left({ }^{\prime}\right)$ denotes differentiation in the direction of maximum velocity gradient $(y)$. The form of Equation 2.5 suggests that the streamwise velocity profile and its derivatives are the primary factors that affect the inviscid stability of plane parallel flows.

The Rayleigh equation is a second-order differential equation that is less general than the Orr-Sommerfeld equation. Flows that are unstable through the analysis of the Rayleigh equation are refered to as "inviscid unstable" which Rayleigh (1880) showed requires an inflection point in the velocity profile. In addition, Fjortoft (1950) demonstrated that inviscid instability requires the velocity gradient $\left(U^{\prime}\right)$ to be a local maximum at the inflection point and there must be a location in the velocity-profile where $U^{\prime \prime}\left(U-U_{p i}\right)<0$, where $U_{p i}$ is the velocity at the inflection point. For example, the boundary layer profile shown in Figure 2.8a is inviscid stable while the profile shown in Figure 2.8b is inviscid unstable due to the presence of an inflection point and the satisfaction of the Fjortoft condition everywhere in the profile except at the 


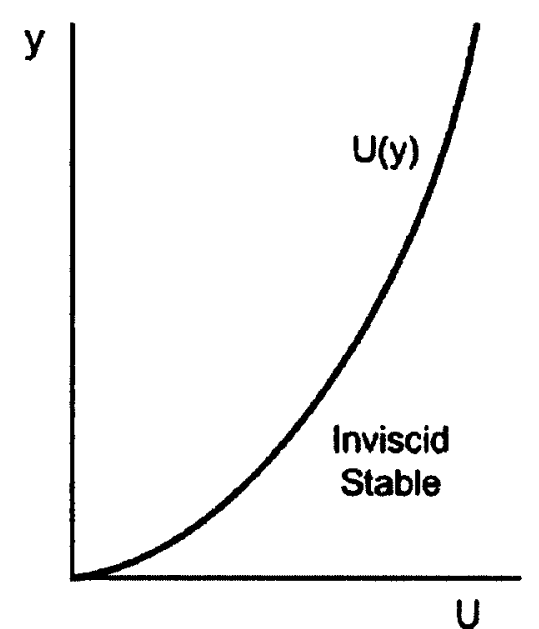

(a)

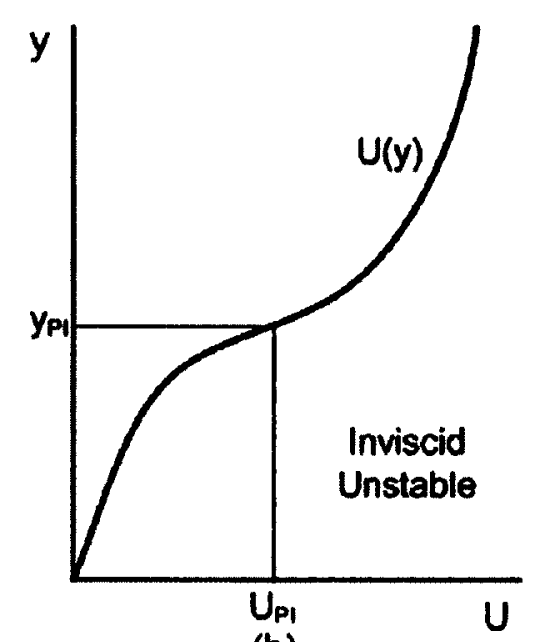

(b)

Figure 2.8: Inviscid stability characteristics of shear layers: $(a)$ an inviscidly stable velocity profile; (b) an inviscid unstable profile.

inflection point (White, 1991).

The eigenvalues of the Rayleigh equation determine the inviscid stability characteristics of a shear layer and provide insight on the initial development of instability in shear layers. Waves of amplified perturbation are often referred to as KelvinHelmholtz (K-H) waves. For example, the initial instability of free shear layer profiles and, occasionally, separated shear layer profiles are assessed through the Rayleigh equation (e.g. Ho and Heurre, 1984; Diwan and Ramesh, 2009). However, this equation is only valid for simple parallel flows where the effects of viscosity and crossstream property variation are not significant.

Following the linear regime, an inviscid unstable shear layer develops a streamwise waviness that manifests itself as stream-normal perturbations of the shear layer. Figure 2.9 models the shear layer as a vortex sheet with vorticity in the clockwise direction, where $y=y(x)$ denotes the stream-normal location of the vortex sheet as a function of the streamwise direction. As the shear layer deforms under the influence of the self-induced velocity field, vorticity is swept away from regions of positive shear-layer slope, where $\frac{\partial y}{\partial x}>0$, towards regions of negative slope, where $\frac{\partial y}{\partial x}<0$ 


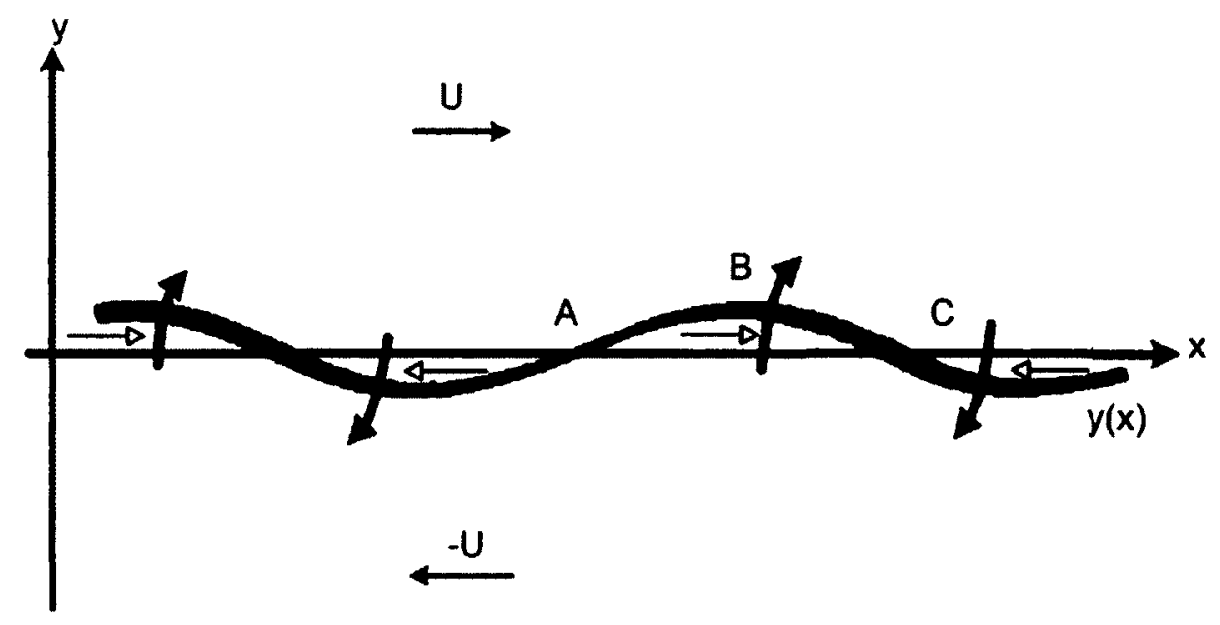

Figure 2.9: Roll-up into Kelvin-Helmholtz vortices (adapted from Drazin (1981)).

(e.g. Drazin, 1981; Bachelor, 1967). This self-induced motion gathers vorticity away from regions such as the one identified as " $\mathrm{A}$ " on the diagram and towards regions such as "C", resulting in periodic streamwise grouping of spanwise (z) vorticity into coherent vortical structures. The resulting vortical structures, often referred to as Kelvin-Helmholtz $(\mathrm{K}-\mathrm{H})$ vortices, are initially two-dimensional structures that contain most of the vorticity within the shear layer (e.g. Acton, 1976; Ho and Huang, 1983).

In separated shear layers, the simplistic model of vortex roll-up mentioned above is affected by the interaction of the shear layer with the wall, as will be elaborated in Section 2.3.4. Spalart and Strelets (2000) describes a mechanism wherein the undeformed shear layer develops instability through wavering (or flapping), which they describe as a state where the streamwise perturbation velocity amplitude $(u)$ at any streamwise location is proportional to the local streamwise velocity gradient in the stream-normal direction $\left(U^{\prime}\right)$ such that $u \propto U^{\prime}$. As the shear layer gradually escapes the confinement of the wall, they observed that the wavering behaviour increases leading to the amplification of unstable K-H waves. In agreement with Spalart and 
Strelets (2000), Na and Moin (1998) observed that the maximum shear stresses are located at the inflection point of a separated shear layer where $\left(U^{\prime}\right)$ is greatest. The streamwise wavering of the shear layer subsequently leads to the formation $\mathrm{K}-\mathrm{H}$ vortices. Bao and Dallman (2003) showed through flow visualization that these primary $\mathrm{K}-\mathrm{H}$ vortices are initially two-dimensional in a laminar separation bubble. Several studies show that at high Reynolds numbers transition may occur suddenly following the formation of the primary $\mathrm{K}-\mathrm{H}$ vortices, thus leaving very little streamwise distance for the formation of higher order instabilities (e.g. Spalart and Strelets, 2000; Tallan and Hourmouziadis, 2002). Despite the unsteady nature of the flow, Tallan and Hourmouziadis (2002) observed that in laminar separation bubbles the flow inside a primary K-H vortex may be laminar. Muti-Lin and Pauli (1996) observed that these primary $\mathrm{K}-\mathrm{H}$ vortices have a dominant role in the mixing of the shear layer such that re-attachment occurs almost immediately following production of these vortices. Bao and Dallman (2003) observed that the primary K-H vortices induce reversed flow which Doligalski et al. (1994) predicted would produce vorticity of the opposite sense at the wall. Several researchers (e.g. Dovgal et al. 1994; Rist and Maucher, 1996; McAuliffe and Yaras, 2007b; Diwan and Ramesh, 2009) observed that the boundary layer formed at the wall by the reversed flow is itself unstable to the T-S instability and this instability interacts with the K-H instability in the separated shear layer.

The instability frequency $(f)$, related to the streamwise wavelength of the K-H vortices, is functionally dependent on the mean cross-stream velocity $(\bar{U})$, and the shear-layer momentum thickness $\left(\theta_{f}\right)$, and is frequently expressed through the nondimensional Strouhal number $S t_{\theta f}=f \theta_{f} / \bar{U}$. In free shear layers, the Strouhal number of the most amplified instability is approximately $S t_{\theta f}=0.032$ based on experiments (Ho and Huang, 1983) and linear stability analysis of a hyperbolic tangent profile using the Rayleigh equations (Michalke, 1964). This nondimensional frequency changes little $(5 \%)$ over a range of velocity-difference ratios $0<R<1$ (e.g. Ho and Huerre, 
Table 2.1: Published Strouhal number ranges in studies of separation bubbles and free shear layers.

\begin{tabular}{ll}
\hline & $\mathrm{St}_{\theta \mathrm{s}}$ \\
\hline \hline McAulliffe and Yaras (2007) & $0.008-0.016$ \\
Muti Lin and Pauley (1996) & $0.005-0.008$ \\
Yang and Voke (2001) & $0.005-0.011$ \\
Talan and Hourmouziadis (2002) & $0.010-0.014$ \\
Ho and Heurre (1984) - free shear layer & 0.016 \\
\hline
\end{tabular}

1984). However, studies of separated shear layers often show a large spread in the Strouhal numbers of vortex shedding. In contrast to free shear layers, in separation bubble studies the Strouhal numbers are formed using the momentum thickness at separation $\left(\theta_{s}\right)$ and freestream velocity $\left(U_{f}\right)$, i.e. $S t_{\theta}=f \theta_{s} / U_{f}$. Table 2.1 summarizes the Strouhal number for several studies. Here, the most amplified Strouhal number of a free shear layer is expressed using the definition for a separated shear layer for ease of comparison with separation-bubble studies. It is evident from Table 2.1 that, while the scatter in the most amplified frequency for a separated shear layer is as high as $50 \%$, the Strouhal numbers values are mostly lower than that of a free shear layer. The reason for this large spread is not well understood. A few researchers suggest that the presence of the nearby wall may play an important role in the spread of Strouhal numbers (e.g. Rist and Maucher, 2002; Diwan and Ramesh, 2009), as will be elaborated in Section 2.3.4.

\subsubsection{Secondary Instability Mechanism}

Following the primary instability, secondary or higher instability modes may develop. The secondary instability mode manifests itself as pairing of the K-H vortices. Figure 
2.10 shows an example of the stages of pairing in a free shear layer. During pairing, one vortex that is perturbed farther toward the high-momentum stream overtakes a vortex immediately preceding it and they subsequently merge to form a larger vortex. The wavelength between the vortices double, yielding a frequency that is half the frequency of the dominant instability frequency (Wille, 1963). This pairing instability is identified as a subharmonic instability of a shear layer and it is an important mechanism by which a free shear layer grows (e.g. Sandham and Adams, 1993; Ho and Huerre, 1984; Moon and Weidman, 1988). Pairing is observed in numerous shear layers including free shear layers, and separated shear layers (Ho and Huerre, 1984; Dovgal et al., 1994; McAuliffe and Yaras, 2007b). Winant and Browand (1974) describe pairing as the instability of an array of finite amplitude vortical structures. Sandham and Adams (1993) provide a simplified model for vortex pairing that considers the kinematics of the vortex rotating around its axis and revolving around the leading vortex. They observed a correlation between the inclination of the vortex about its axis and the stages of vortex pairing. Winant and Browand (1974) describe the pairing process as being, at best, intermittent, and stochastic variations in the strength and spacing of the vortices lead to variability in the streamwise location of pairing.

Separated shear layers typically transition too soon to develop pairing instability (e.g. Spalart and Strelets, 2000; Tallan and Hourmouziadis, 2002). However, recent numerical studies of separation bubbles by McAuliffe and Yaras (2007b) suggest that, for separated shear layers, the pairing mechanism is observed for low Reynolds numbers $\left(R e_{\theta s} \approx 100\right)$ and suppressed at higher Reynolds numbers.

\subsubsection{Breakdown into Turbulence}

The process of transition into turbulence in a free shear layer begins with the developement of spanwise waviness in the velocity profile (e.g. Bernal, 1981; Lin and 

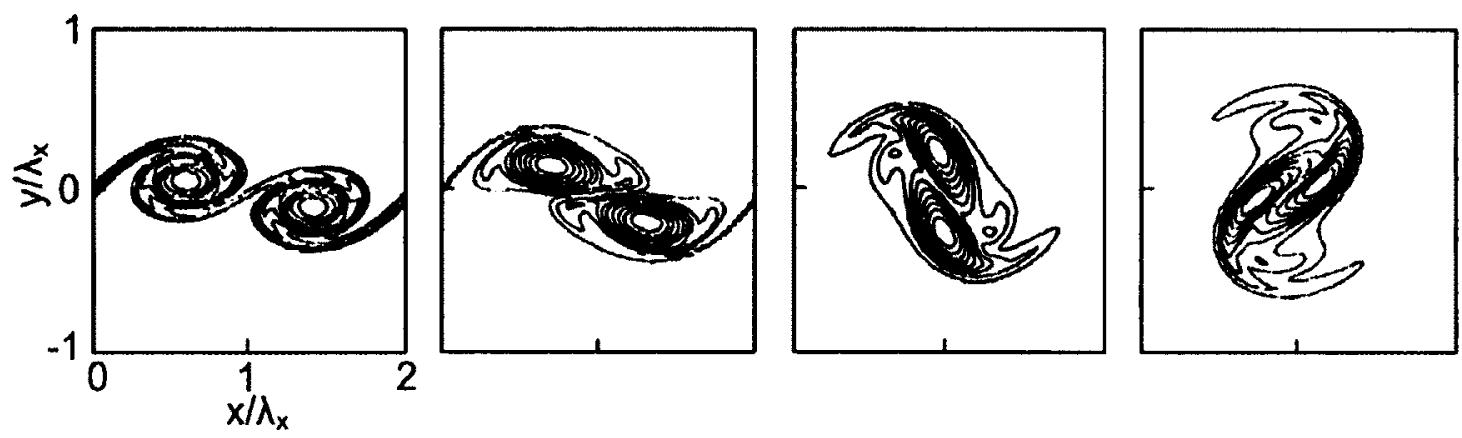

Figure 2.10: Stages of the pairing instability illustrated through spanwise vorticity isocontours, where $\lambda_{x}$ is the streamwise wavelength of the K-H vortices (Sandham, 1993).

Corcos, 1981). However, the development of this three-dimensionality is not well understood. Some researchers (e.g. Craik, 1971,1980) describe a mode similar to those observed in boundary layers, as described in Section 2.2.2, such that the shed vor-. tices self-induce $\Lambda$-shaped structures which evolve into hairpin-like vortical structures that generate high-shear regions where localized regions of turbulent flow are produced. Other researchers, such as Pierrehummbert and Widnell (1982), believe that the $\mathrm{K}-\mathrm{H}$ vortices are themselves unstable and they describe mechanisms by which these vortices deform and become three-dimensional. They propose two modes, the helical and translative modes, as mechanisms of $\mathrm{K}-\mathrm{H}$ vortex deformation. Claufield and Peltier (2000) and McAuliffe and Yaras (2007b) assert that the high shear braids between vortices act as sites of disturbance amplification that manifests as spanwise redistribution of streamwise vorticity. Earlier numerical work by Lin and Corcos (1981) demonstrates that such a flow development forms three-dimensional, counterrotating vortices (Figure 2.11) that cause the breakdown of the shear layer. Claufield and Peltier (2000) and Estevadeordal and Kleis (2002) describe the development of streamwise vortices that wrap around the K-H vortices. They noted the creation of streamwise vortices within braids between $\mathrm{K}-\mathrm{H}$ vortices act to groove out spanwise 


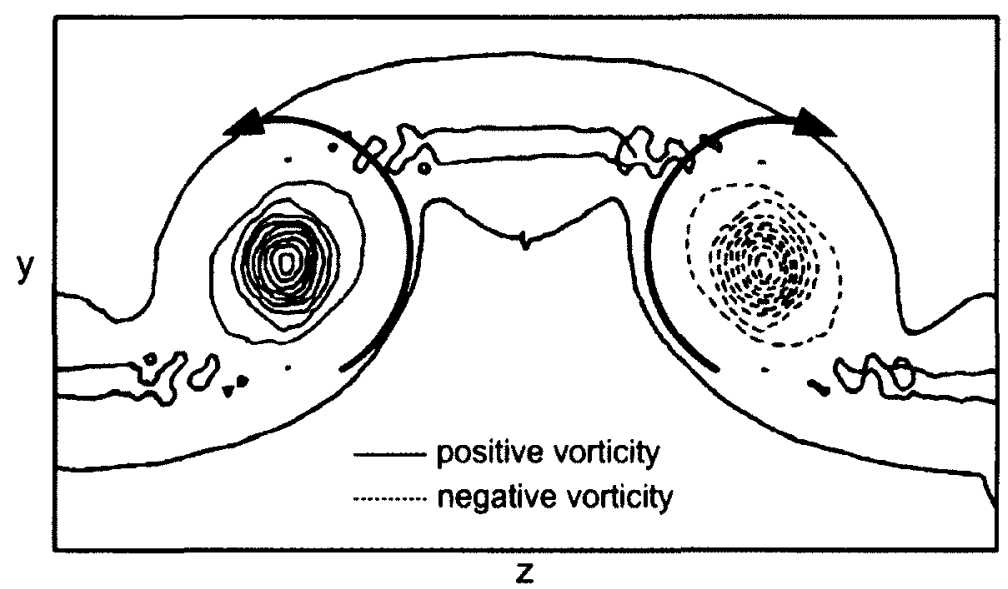

Figure 2.11: Development of streamwise vortices (shown using streamwise vorticity contours) in the stream-normal $(y-z)$ plane (adapted from Lin and Corcos,1981).

undulation on the $\mathrm{K}-\mathrm{H}$ vortices.

The spatial organization of these streamwise vortices is observed to be a function of Reynolds number. Williamson (1995) observed that the pattern of streamwise vortices for flows over cylinders depends on the Reynolds number based on the cylinder diameter $\left(R e_{D}\right)$, as shown in Figure 2.12. He observed "type A" instabilities consisting of a ribbed pattern of staggered streamwise vortices at $R e_{D}=200$ and "type B" instabilities of finer streamwise vortices of more consistent spacing that weave between the spanwise rollers at $R e_{D}=270$. These patterns are schematically shown in Figure 2.12. Claufield and Peltier (2000) and McAuliffe and Yaras (2007) noted that these streamwise vortices play a fundamental role in the three-dimensional breakdown of shear layers into turbulence.

Similarly, breakdown in separated shear layers is shown to be contingent on the development of spanwise deformation in the shear layer. Yang and Voke (2001) show that following the development of the primary $\mathrm{K}-\mathrm{H}$ vortices, three-dimensional streamwise vortices form at about $50 \%$ of the time-mean reattachment length from 


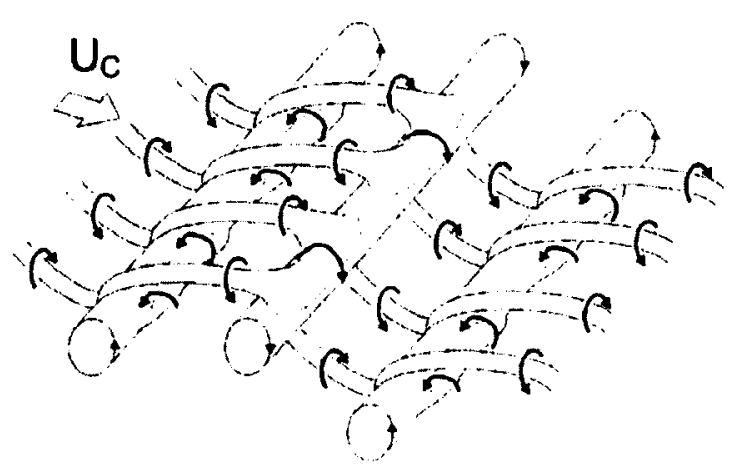

(a)

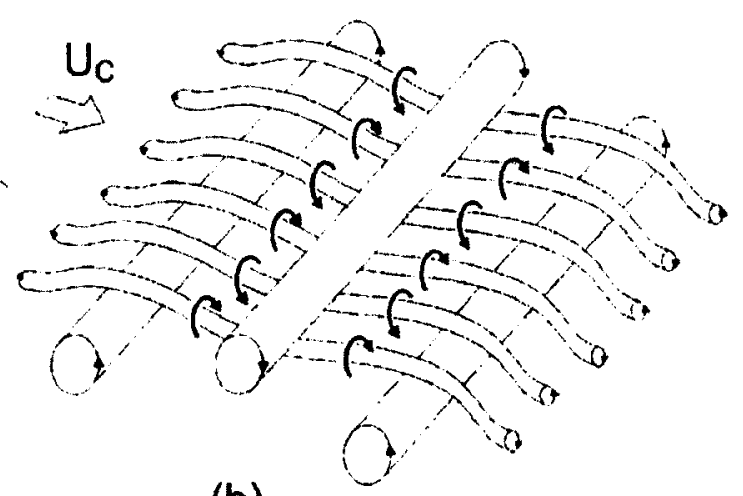

(b)

Figure 2.12: Streamwise vorticity patterns for (a) type A and (b) type B instabilities (Baily et al., 2002).

separation. Likewise, Alam and Sandham (2000) observed the formation of $\Lambda$ vortices during the transition of the shear layer into turbulence similar to those observed in boundary layers. McAuliffe and Yaras (2007b) observed that three-dimensional vortical structures are created at the braids between K-H vortices where the local high shear stress acts to amplify perturbations. They observed that complete three-dimensional breakdown occurs one to two $\mathrm{K}-\mathrm{H}$ wavelengths downstream of the formation of these three-dimensional structures. Most recently, Brinkerhoff and Yaras (2011) demonstrated that the spanwise deformation responsible for the threedimensional breakdown of the separated shear layer in fact originates from the upstream boundary layer. This suggests that transition in the separated shear layer is also affected by the T-S instability of the upstream boundary layer.

\subsubsection{Effect of Proximity to a Wall}

As mentioned earlier, the main difference between a separated shear layer and a free shear is the presence of a no-slip wall beneath the separated shear layer. Therefore, it is reasonable to suspect that the proximity of the shear layer to the no-slip wall 


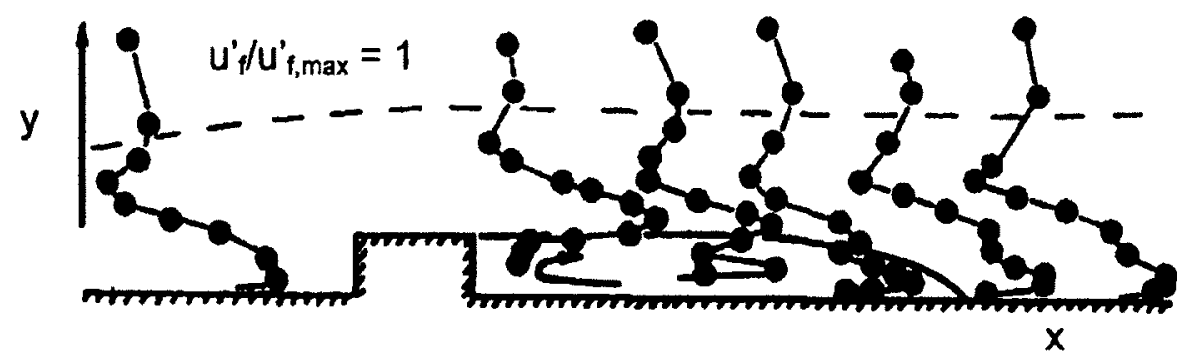

Figure 2.13: The development of the streamwise velocity fluctuation profile during the transition of a boundary layer velocity profile to a separated shear layer velocity profile (Dovgal et al., 1993).

plays a significant role in many of the differences in instability development observed in separated shear layers relative to free shear layers.

Studies by Taghavi and Wazzan (1974), Nayfeh, Ragab and Masad (1990), Das (1998) and Boiko et al. (2002) suggest that proximity to a wall suppresses the onset of instability. Dovgal et al. (1993) gives an excellent discussion of shear layers in close proximity to walls. During the process of flow separation triggered by a hump, they observed that the upstream streamwise velocity fluctuation profile transitioned into a new profile for a separated shear layer, as shown in Figure 2.13. The upstream streamwise velocity fluctuation profile is characteristic of the T-S instability, as discussed in Section 2.5, while the downstream streamwise velocity fluctuation profile is influenced by the instability of the upstream boundary layer and the K-H instability of the separated shear layer. Their experimental studies reveal three extremas in streamwise velocity fluctuation profile of the separated shear layer: one inside the bubble, one within the shear layer profile and one at the outer periphery of the viscous layer. 
Rist and Maucher (2002) performed a linear stability analysis for a modified hyperbolic tangent velocity profile, described by:

$$
U(y)=\tanh \left(a\left(y-y_{s}-\left(y-y_{s}\right)^{r}\right)\right)
$$

where the parameter $y_{s}$ represents the distance of the shear layer from the wall. By repeating the analysis with various values of $y_{s}$, they determined that proximity to a wall dampens the onset of the inflectional instability mode at near-wall proximities. This is seen in Figure 2.14, which shows the streamwise velocity fluctuation profile for two values of $y_{s}$; the profile in Figure 2.14a shows a far-wall configuration while the profile on Figure 2.14b shows a near-wall configuration. When the shear layer is located far from the wall (Figure 2.14a), the largest perturbation amplitude occurred near the center of the shear layer, suggesting that the K-H instability mode has a larger influence in the overall instability of the separated shear layer. When the shear layer is located close to the wall (Figure 2.14b), the velocity profile experienced its highest magnitude perturbation near the wall, suggesting a larger influence of the T-S modes. This suggests that the wall height adjusts the relative influence of the two instability modes on the overall instability of the separated shear layer. Furthermore, they observed that the most-unstable wavenumber reduces in close proximity to the wall.

Diwan and Ramesh (2009) performed a linear stability analysis of a family of piecewise-linear velocity profiles using the Rayleigh equation and observed that there is a reduction in the most-amplified frequency for profiles observed in shear layers in close proximity to the wall. To complement their linear stability analysis, Diwan and Ramesh (2009) also correlated the most amplified disturbance frequency of a separation bubble to the Reynolds number based on the inflection-point height from the 


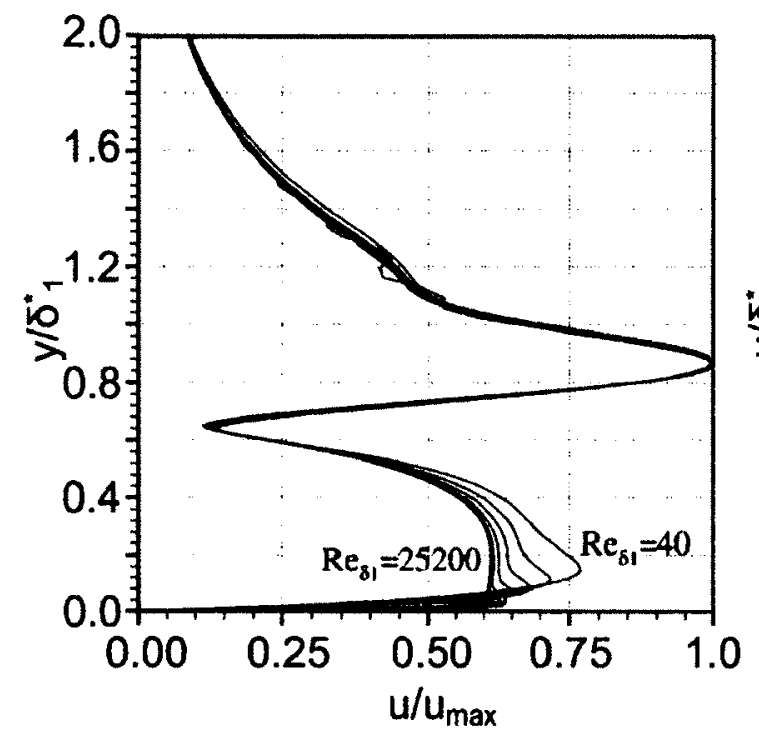

(a)

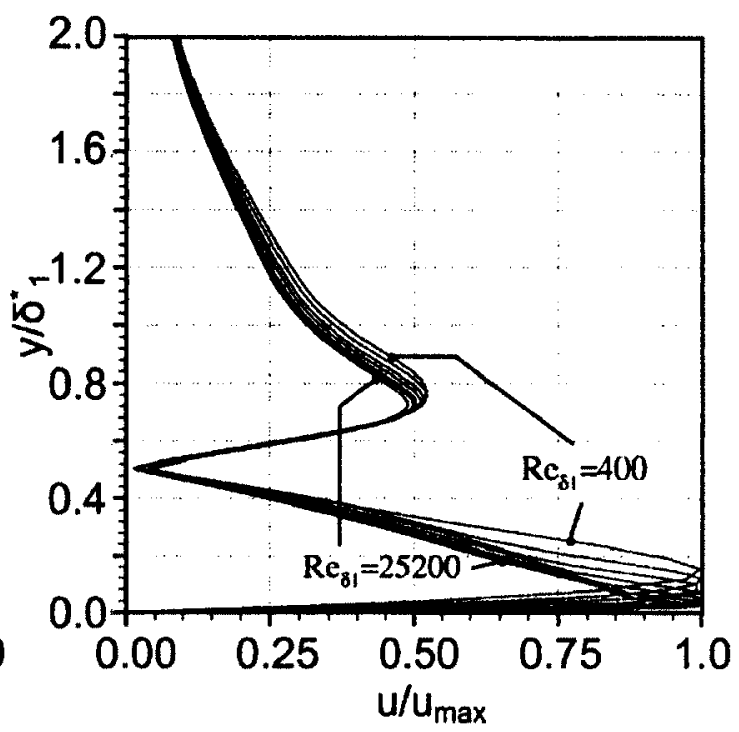

(b)

Figure 2.14: Streamwise perturbation velocity profile $(\mathrm{u})$ profiles for (a) $\mathrm{y}_{\mathrm{s}} / \delta_{1}^{*}=0.70(\mathrm{~b}) \mathrm{y}_{\mathrm{s}} / \delta_{1}^{*}=0.55$. Wall-normal height $(\mathrm{y})$ is normalized by shear layer displacement thickness $\left(\delta_{1}^{*}\right)$ (Rist and Maucher, 2002).

wall. They postulated that the relevant quantities that affect the most amplified frequency, $f_{M A}$, are the streamwise velocity at the inflection point; wall-normal position of the inflection point $\left(y_{i n}\right)$, viscosity, and the vorticity thickness $\delta_{\omega}$ defined as:

$$
\delta_{\omega}=\left(U_{1}-U_{2}\right) /(d U / d y)_{\max }
$$

Using the Buckingham Pi theorem they obtained a functional relationship between the Reynolds number based on inflection point height and a non-dimensional frequency:

$$
\frac{f_{M A}\left(y_{i n}^{2}+\delta_{\omega}^{2}\right)}{\nu} \sim \frac{U_{i n} y_{i n}}{\nu} \sqrt{\frac{y_{i n}}{\delta_{\omega}}}
$$

Diwan and Ramesh plotted the most amplified instability frequency with their modified Reynolds number parameter and observed qualitative agreement with their linear 


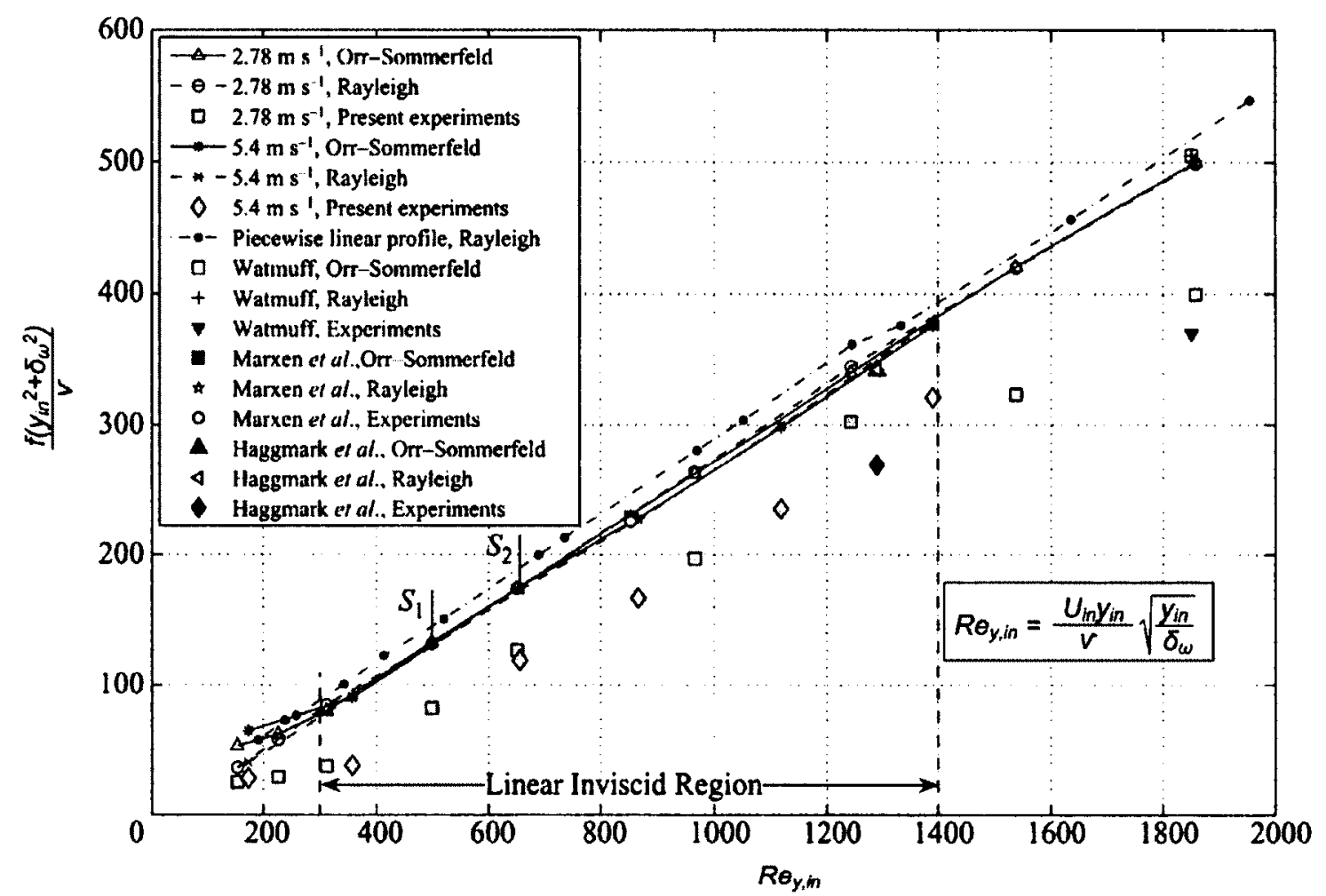

Figure 2.15: Scaling relation for the most amplified frequency in the separation bubble (Diwan and Ramesh, 2009).

stability analysis, as shown in Figure 2.15. However, their correlation generally overpredicted the frequencies compared to experimental results. Moreover, the validity of the inviscid analysis for a piecewise linear profile that does not truly have an inflection point is questionable and the conclusion that the effect can be explained using inviscid theory contradicts the work of Rist and Maucher (2002), who view the effect as fundamentally viscous. Therefore, further investigations are required to investigate the mechanism for the reduction in the dominant instability frequency with wall proximity.

$\mathrm{Ni}$ and Armaly (2002) studied the instability and heat transfer properties of shear layers over a backward facing step for differing heights for $R e_{\theta}=343$ at the location of the step. For all flows, a region of recirculation developed below the shear layer. 
However, as the step height is increased, the fraction of the flow entering a secondary recirculation zone increased. The wall shear stress following reattachment was observed to increase at larger step heights. Bailey et al. (2001) studied the influence of wall proximity on the instability of the shear layer formed in the wake of a square cylinder. They observed that for low separations of the square cylinder from the wall, the growth of the instabilities is suppressed. Furthermore, they observed that proximity to the wall produces more oblique vortex shedding and reduce the spanwise coherence of these vortices.

\subsubsection{Effect of Cross-Stream Property Gradients}

In addition to wall proximity, the presence of cross-stream property gradients can affect the stability of a shear layer. Although the emphasis of the present study is on separation bubbles in the context of aerodynamic optimization of gas turbine engines, interest in using supercritical fluids in power generation applications has led to increased research attention on shear layers with significant cross-stream thermodynamic property gradients. Thermally supercritical fluids are proposed to be used in turbine stages and nuclear reactor fuel bundles and, as will discussed in Section 2.4, these flows experience significant property gradients due to mild heating or cooling at solid boundaries of the flow path. To facilitate the discussion in Chapter 5, where shear layers composed of supercritical water are studied, the following section discusses literature pertaining to flows with cross-stream property gradients. Although the study of supercritical shear layers in Chapter 5 includes cross-stream variation in density, viscosity, thermal conductivity, and isobaric heat capacity, the present review is limited to the role of cross-stream variations in density and viscosity because these properties are generally accepted as playing the most significant role on the transition of the shear layers into turbulence (e.g. Shafer and Herwig, 1995).

Some of the earliest studies that investigated the stability of density-stratified 
shear layers in the presence of a gravitational field were performed by Taylor (1931) and Goldstein (1931). They applied linear stability theory on shear layers with crossstream density gradients and derived the Taylor-Goldstein equation:

$$
v^{\prime \prime}+\left(\frac{N^{2}}{(U-c)^{2}}-\frac{U^{\prime \prime}}{(U-c)}-\alpha^{2}\right) v=0
$$

where $N=\sqrt{(g / \rho)(-d \rho / d y)}$ is the buoyancy frequency, $g$ is the gravitational acceleration acting in the cross-stream direction, $U$ is the baseflow velocity profile, $v(y)$ is the stream-normal velocity perturbation mode shape, $\alpha$ is the streamwise wavenumber, $c$ is the perturbation phase velocity, and the prime $\left({ }^{\prime}\right)$ denotes differentiation in the direction of maximum velocity gradient (y) (Lawrence et al., 1991). Taylor (1931) studied the stability of several velocity profiles with differing levels of density stratification and observed that the range of stable waves and the mode shapes of instability are dependent on the density profile. Drazin and Reed (1981) provides a good review on the role of density stratification on the stability of shear layers. For flows where the lighter fluid is above the heavier fluid, the influence of a downward-directed gravitational force is to stabilize the shear layer.

To assess the level of flow stratification Lawrence et al. (1991) use the nondimensional Richardson number, defined as:

$$
J=g \frac{\Delta \rho}{\rho_{2}} \frac{\Delta U}{(d U / d y)_{\max }} \frac{1}{\Delta U^{2}}
$$

where, $\Delta \rho=\rho_{1}-\rho_{2}, \Delta U=U_{1}-U_{2}, \rho_{1}$ is the upper stream density, and $\rho_{2}$ is the lower stream density. They studied the Taylor-Goldstein equation for selected shear layer profiles and plotted a stability diagram for a range of flow Richardson numbers $(J)$, shown in Figure 2.16. They predicted that in shear flows with low values for the real component of phase speed of unstable perturbations $\left(c_{r}<0.25\right)$, Holmboe 


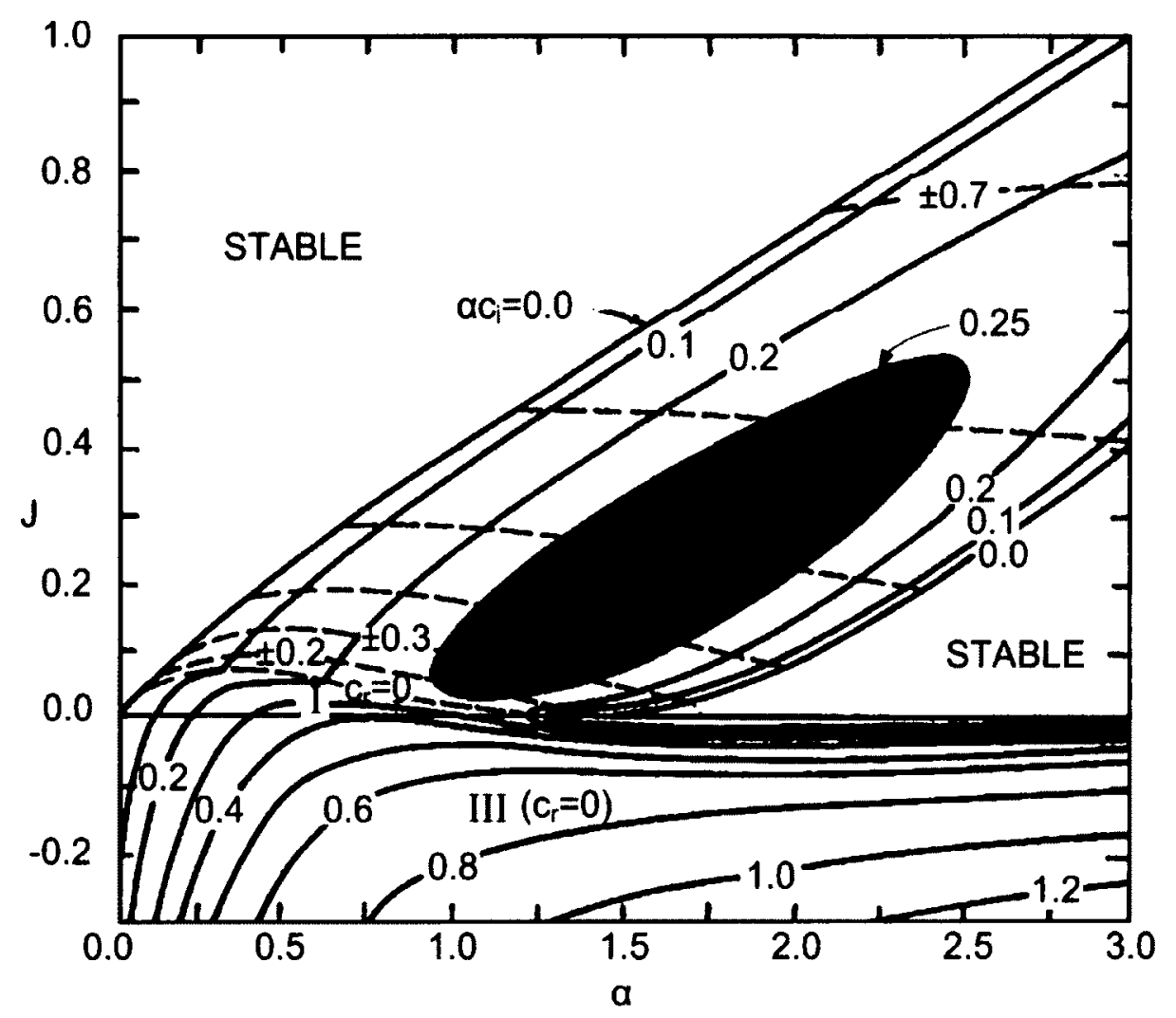

Figure 2.16: Stability plots for the Taylor Goldstein equation showing contours of unstable waves. Region bounded by $\alpha c_{i}=0.25$ (coloured in blue) are prone to the Holmboe instability (Lawrence et al., 1991).

instability can form and co-exist with the $\mathrm{K}-\mathrm{H}$ instability.

The Holmboe instability occurs in shear layers with increasing density in the direction of a gravitational force (Holmboe, 1960). Two trains of interfacial waves of equivalent strengths develop and travel in oppose directions above and below the mean density interface, as shown schematically in Figure 2.17a (Lawrence et al., 1991). For low levels of density stratification, often quantified by $J$, the shear layer rolls up into the well known K-H vortices. However, above a threshold value of $J$, given by regions enclosed in $c_{r}<0.25$ in Figure 2.16 , the shear layer ceases to rollup and instead Holmboe waves amply and eject long thin plumes above and below 
the shear layer (Smyth et al., 1988; Lawrence et al., 1991). The spanwise-oriented Holmboe vortices that subsequently develop are visualized by two counter-rotating vortex segments above and below the shear layer, such as in Figure $2.17 \mathrm{~b}$ (Carpenter et al., 2010; Alexakis, 2009). Alexakis (2009) numerically studied the linear and nonlinear evolution of stratified shear layers and observed the presence of $\mathrm{K}-\mathrm{H}$ and Holmboe vortices. Kelvin-Helmholtz vortices are observed to develop sooner than the Holmboe vortices and they have kinetic energies approaching ten times those of the Holmboe vortices (Alexakis, 2009). They describe the creation of Holmboe vortices as being preceded by formation of cusps that subsequently break-up into Holmboe vortices. Furthermore, Lawrence et al. (1991) performed experimental studies of flows with Richardson numbers between 0.05 and 0.3 and observed flow stabilization for higher Richardson numbers and formations of cusps that are possibly the footprint of Holmboe instability. Lee and Claufield (2001) numerically studied the instability of shear layers with cross-stream density gradients in a gravitational field. They introduced a distinction between two types of instabilities in shear layers with crossstream density gradients, namely Taylor instabilities and Holmboe instabilities. The latter occurs only for sharply-varying cross-stream density profiles while the former occurs for mild-gradient density profiles. In addition to $\mathrm{K}-\mathrm{H}$ vortices, they observed smaller pairs of counter-rotating vortices. Lee and Claufield referred to the smaller vortices as Taylor vortices. However, the distinction between Holmboe vortices and Taylor vortices remains ambiguous and these vortices may in fact be formed by the Holmboe instability described by other researchers (Holmboe, 1962; Smyth et al., 2006; Lawrence et al., 1991; Smyth and Peltier, 1991).

Claufield and Peletier (2000) performed numerical simulations of flows with and without cross-stream density gradients and observed that shear layers with density gradients show earlier development of three-dimensional streamwise vortices and more 


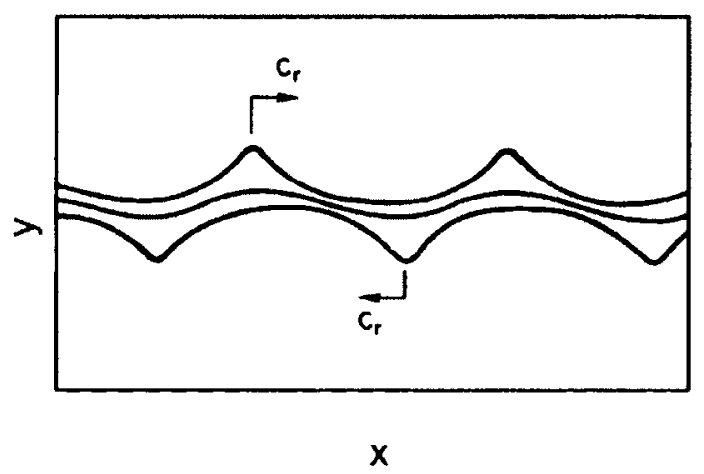

(a)

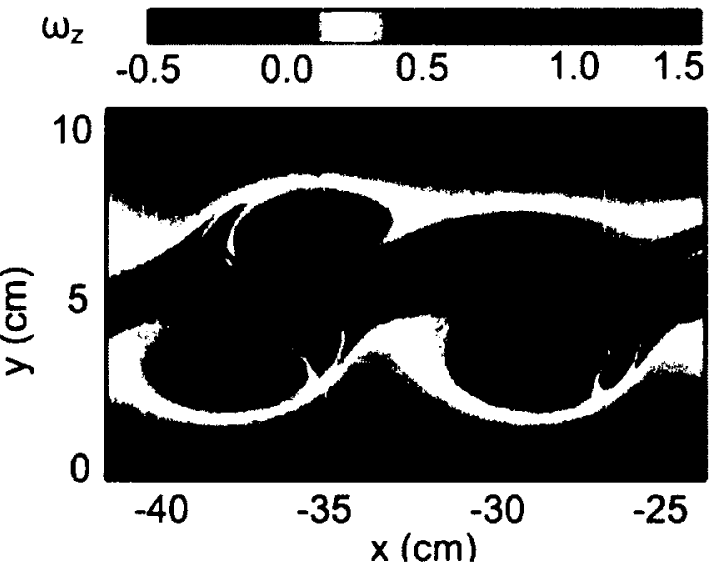

(b)

Figure 2.17: (a) Schematic showing the formation of Holmboe vortices (Lawrence et al., 1991). (b) Holmboe vortices shown through flood plots of spanwise vorticity where density variation is shown using contour lines (reproduced from Carpenter et al., 2010).

numerous streamwise vortices. Their numerical results were validated against a linear stability analysis, which accounted for cross-stream variation in density, that confirmed their observed trend of increased streak spacing. However, they do not provide an explanation for this increased number of streaks. Smyth (2005) studied the three-dimensional instability mechanisms of the $\mathrm{K}-\mathrm{H}$ and the Holmboe instabilities and concluded that streamwise vortices can form accompanying $\mathrm{K}-\mathrm{H}$ vortices and Holmboe vortices, as shown by the isocontours of streamwise velocity in Figure 2.18. They observed that streamwise vortices accompanying Holmboe vortices are less coherent, however, surprisingly persistent through the simulation times. They observed a correlation between the growth of these secondary vortices and the Richardson number, with growth rate decreasing with higher Richardson numbers. They provide a possible explanation for the instability based on a potential energy budget argument.

Wazzan and Keltner (1972) examined the role of viscosity variation of water due to wall heating on the spatial stability of Falkner-Skan boundary layer profiles by 


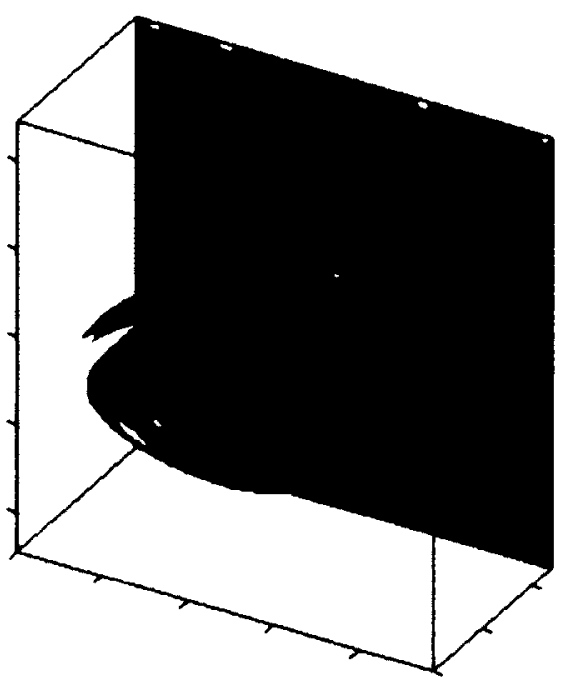

(a)

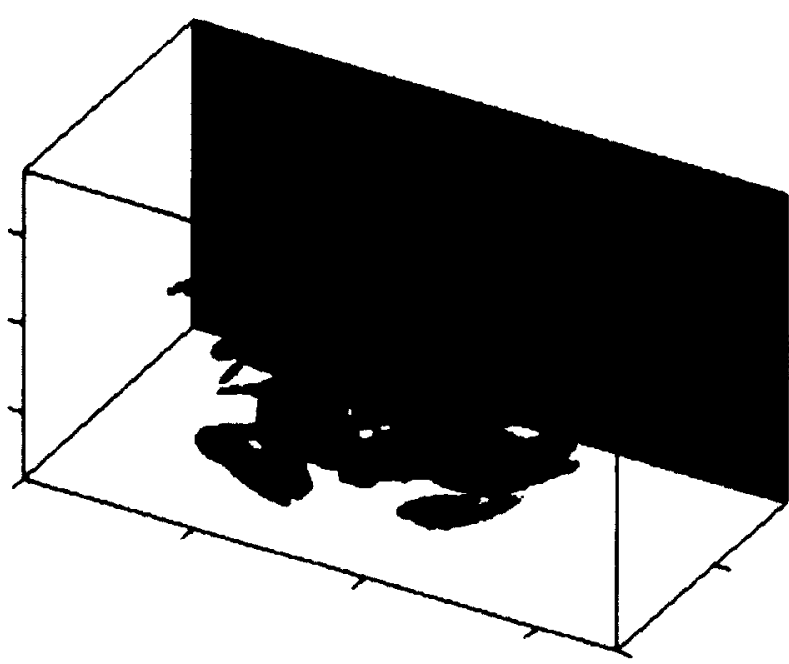

(b)

Figure 2.18: Streamwise vortices in a shear layer with cross-stream property gradients accompanying (a) K-H vortices (b) Holmboe vortices (red: positive-sense streamwise vorticity; blue: negative-sense streamwise vorticity)(Smyth, 2005).

studying the Orr-Sommerfeld equation modified for the effects of viscosity variation. They observed that viscosity profiles produced by wall heating stabilize the flow-field while cooling destabilizes the flow-field. These results are in agreement with those of Hauptman (1967) who developed correlations for the critical Reynolds number to account for the effects of wall heating on flow instability through modifications of fluid viscosity.

Shafer and Herwig (1995) studied the role of a non-dimensional densitytemperature gradient $\left(K_{\rho T}\right)$ and viscosity-temperature gradient $\left(K_{\mu T}\right)$ on the stability of heated and cooled flows. They observed that for fluids such as air, where $\left(K_{\rho T}\right)$ is negative and $\left(K_{\mu T}\right)$ is positive, both factors contribute to the stabilization of the flow-field during cooling and destabilization during heating. For fluids such as water, where $\left(K_{\rho T}\right)$ is positive and $\left(K_{\mu T}\right)$ is negative, the flow-field is stabilized by heating and destabilized by cooling. The decreased stability of air flow due to heating has 
been observed experimentally by Liepmann and Fila (1947).

Azih et al. (2012a) suggests that cross-stream viscosity gradients have significant influence on the stability of supercritical fluid boundary layers on heated walls. They argue that the variation in viscosity profile due to heating of the fluid reduces the eddy turn-over time in the boundary layer (Azih et al., 2012a). Following the optimal perturbation theory (Baig and Chernyshenko, 2004), this is accompanied by a reduction in the spanwise wavelength of steaky structures (Azih et al., 2012a). They observe that this effect produces more numerous streaks in the boundary layers thereby improving mixing.

\subsection{Thermally Supercritical Flows}

Heating a fluid above its critical pressure $\left(P_{c}\right)$ through its critical temperature $\left(T_{c}\right)$ causes rapid, single-phase, changes in its thermophysical properties (Tour, 1891; Pioro et al., 2003). These property variations are shown in Figure 2.19 for water at $24 \mathrm{MPa}$. In recent years, there has been considerable interest in using fluids near a thermodynamic supercritical state for power-generation cycles. For example, one concept for the Generation- 4 nuclear reactor uses supercritical water as the coolant to enhance thermal efficiency and simplify the cycle by avoiding two-phase heat transfer. Numerous studies indicate regimes of enchanced and deteriorated heat transfer in heated supercritical fluid flows (e.g. Yamagata, 1971; Hall and Jackson, 1969; Bae et al., 2005; Azih et al., 2011). However, the mechanisms responsible for these heat transfer variations are not fully understood. Azih et al. (2011) suggest that hydrodynamic stability and turbulence properties of the flows are fundamentally responsible for these variations in heat transfer. This section discusses literature on the hydrodynamic stability and heat transfer properties of supercritical fluids to provide a context for the discussion of the results of the present study on layers involving supercritical 


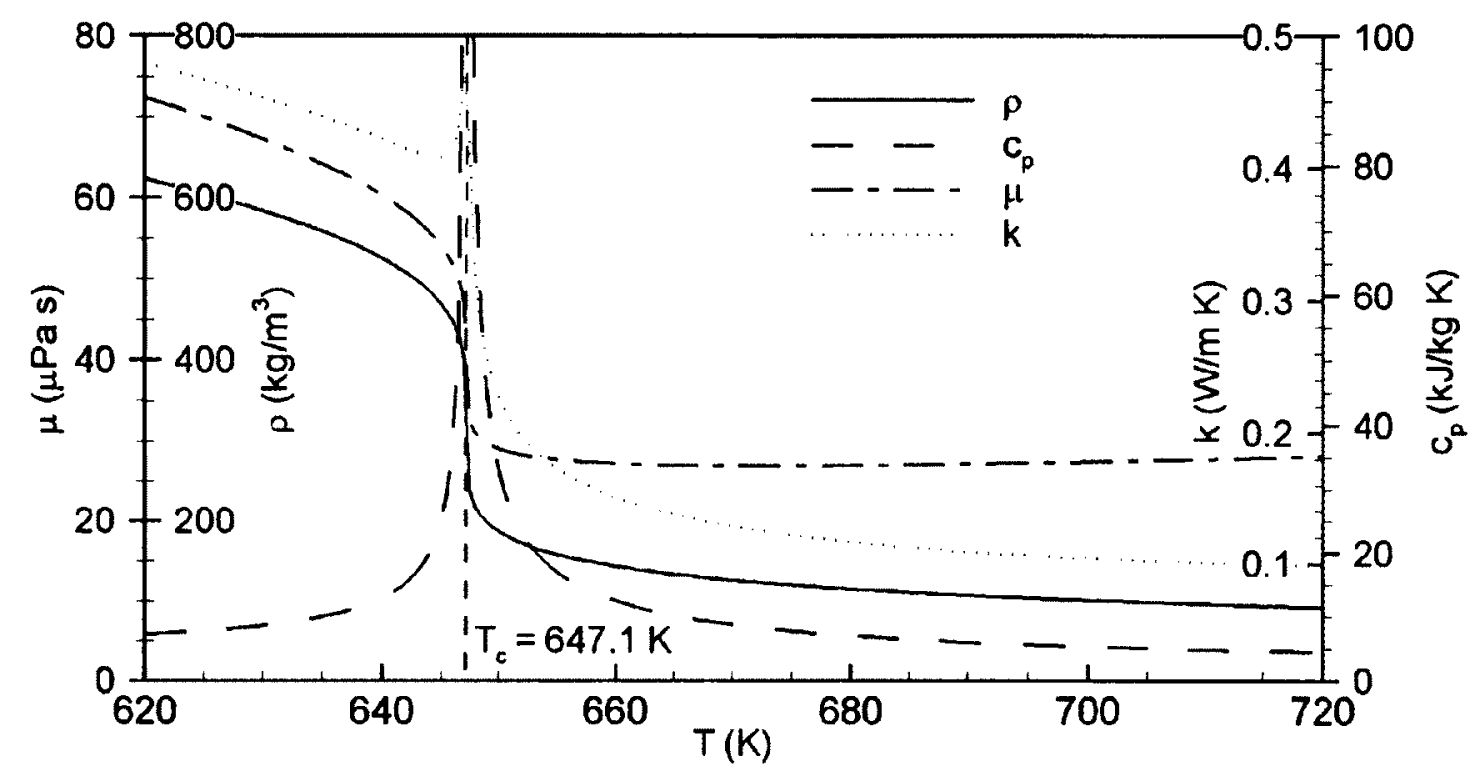

Figure 2.19: The variation of density $(\rho)$, dynamic viscosity $(\mu)$, thermal conductivity $(k)$ and isobaric specific heat capacity $\left(c_{p}\right)$ for water at the critical pressure at $\mathrm{P}_{\mathrm{c}}=22.1 \mathrm{MPa}(\mathrm{NIST}, 2011)$.

fluids provided in Chapter 5.

A number of researchers have studied supercritical-fluid heat transfer with numerical methods that use turbulence models such as mixing models (e.g. Bellmore and Reid, 1983; Howell and Lee, 1999), the $k-\epsilon$ eddy viscosity model (e.g. Koshizuka et al., 1995), and Reynolds stress models (e.g. He et al., 2004). Although each model may provide good agreement for their geometry of calibration, the predictive performance of each model varies significantly from one model to the next. Bae and Yoo (2005) suggested that improved turbulence models for supercritical flows require detailed data on the structure of flow turbulence through experiments or direct numerical simulations (DNS). In their DNS of vertical channel flows with supercritical carbon dioxide, they observed deteriorated heat transfer in areas of low turbulence activity. They observed that buoyancy contributes to the enhanced heat transfer for 
flows in the direction of the gravitational field primarily by enhancing turbulence activity.

Azih et al. (2012a,b) studied the role of property variation of supercritical fluids due to wall heating in a boundary layer in the absence of buoyancy forces using DNS. They observed a correlation in the enhancement of heat transfer in the boundary layer with reduced spanwise wavelength of the near wall streaks. They propose that the smaller wavelengths of these streaks lead to more numerous streaks leading to better wall-normal mixing and hence better heat transfer.

In some applications, supercritical fluid flows can develop a shear layer configuration. There are a few published studies that discuss the stability of these shear layers. Miller et al. (1999) performed a DNS of supercritical nitrogen-heptane in the absence of a gravitational field. They observed that during the formation of K-H vortices, opposite sense vorticity develop which they attribute to "density gradient effects". Later, Okong'o and Bellan (2003) performed an inviscid linear stability analysis of supercritical nitrogen-heptane mixing layers in the absence of a gravitational field. Their study shows that the most unstable wavelength for shear layers is strongly dependent on the density ratio $\left(\rho_{2} / \rho_{1}\right)$ between the high momentum and low momentum stream with higher density ratios having lower streamwise wavelengths $\left(\lambda_{x}\right)$ of the most amplified instability. Following their stability analysis, they simulated a shear layer with $\rho_{2} / \rho_{1}=12.88$ and perturbed it with a disturbance of streamwise wavelength $\lambda_{x} \approx 4 \delta_{\omega}$ and $\lambda_{x} \approx 8 \delta_{\omega}$ where $\delta_{\omega}$ is the vorticity thickness of the shear layer. They observed that the vortices produced by the higher and lower wavelength perturbation differed significantly. Although Okong'o and Bellan did not specifically mention this, the vortices observed in the smaller wavelength perturbation $\left(\lambda_{x} \approx 4 \delta_{\omega}\right)$ case resemble the Holmboe wave patterns studied by Alexakis (2009). 


\section{Chapter 3}

\section{Computation Method}

\subsection{Overview}

This chapter describes the details of the numerical method and the setup of the computational domains for the simulations performed in this study. Section 3.2 describes the numerical method adopted by the commercial software package ANSYS CFX in the solution of the Navier-Stokes equations. Section 3.3 describes the computational configuration used to study two-dimensional shear layers in close proximity to a wall and three-dimensional shear layers in supercritical fluids with cross-stream property gradients. Finally, Section 3.4 describes the steady and transient potential flow algorithms used to explain the trends in delayed transition and vorticity interaction in the shear-layer in close proximity to a wall as observed through the solution of the Navier-Stokes equations. 


\subsection{Computational Method}

\subsubsection{Governing Equations of Fluid Flow}

The continuity, momentum and energy equations, shown in tensor notation in Equations $3.1,3.2$ and 3.3 , respectively, are the transport equations that describe fluid motion. These equations are solved in ANSYS CFX in their conservative form with velocity components $\left(U_{i}\right)$, static pressure $(P)$ and total enthalpy $\left(h_{0}\right)$ as the primitive variables.

$$
\begin{gathered}
\frac{\partial \rho}{\partial t}+\frac{\partial}{\partial x_{i}}\left(\rho U_{i}\right)=0 \\
\frac{\partial}{\partial t}\left(\rho U_{i}\right)+\frac{\partial}{\partial x_{i}}\left(\rho U_{j} U_{i}\right)=-\frac{\partial P}{\partial x_{i}}+\frac{\partial}{\partial x_{i}}\left(\mu\left(\frac{\partial U_{i}}{\partial x_{j}}+\frac{\partial U_{j}}{\partial x_{i}}\right)\right) \\
\frac{\partial}{\partial t}\left(\rho h_{0}\right)-\frac{\partial P}{\partial t}+\frac{\partial}{\partial x_{i}}\left(\rho U_{i} h_{0}\right)=\frac{\partial}{\partial x_{j}}\left(k \frac{\partial T}{\partial x_{j}}\right)+\frac{\partial}{\partial x_{i}}\left(U_{i} \mu\left(\frac{\partial U_{i}}{\partial x_{j}}+\frac{\partial U_{j}}{\partial x_{i}}\right)\right)
\end{gathered}
$$

These equations are coupled with the equation of state, which relates the density to the pressure and temperature, and the constitutive equation, which relates the static enthalpy to pressure and temperature. The fluids used in this study include air as an ideal gas and supercritical water. In the instance of air as the working fluid, the ideal gas law is used as the equation of state and a linear relationship is used between the enthalpy $(h)$ and temperature $(T)$ :

$$
h-h_{r e f}=c_{p}\left(T-T_{r e f}\right)
$$

Here, $c_{p}$ is the specific isobaric heat capacity, $T_{r e f}$ and $h_{r e f}$ are the temperature and static enthalpy at a reference state and the total enthalpy is related to the static enthalpy through:

$$
h_{0}=h+0.5\left(U^{2}+V^{2}+W^{2}\right)
$$


Supercritical water shows significant changes in density $(\rho)$, viscosity $(\mu)$, thermal conductivity $(k)$ and specific isobaric heat capacity $\left(c_{p}\right)$ over a narrow range of temperature values. The International Association for the Properties of Water and Steam (IAPWS) databases provided in ANSYS CFX are used to generate look-up tables for these properties, with the relevant thermophysical properties tabulated as a function of temperature and pressure. The thermodynamic grid in this look-up table is sized such that the temperature and pressure ranges are approximately four times the ranges expected in the present simulations. During the solution of the NavierStokes equations, the ANSYS CFX solver linearly interpolates within this look-up table. Temperature and pressure increments of $0.2 \mathrm{~K}$ and $0.1 \mathrm{MPa}$, respectively, are established to be sufficient to provide accurate results based on linear interpolation between the entries in the look-up table. Details of the sizing and validation of this thermodynamic grid is presented in Appendix A.

\subsubsection{Discretization of the Partial Differential Equations}

To numerically solve the governing equations of fluid flow in ANSYS CFX, the spatial domain is discretized into finite hexahedral volumes. Figure 3.1 shows an example of a finite volume created through the median-dual vertex scheme utilized by ANSYS CFX. In the median-dual vertex scheme, finite-volume surfaces are created by connecting face centers $(\mathrm{F})$, edge midpoints $(\mathrm{M})$ and cell centroids $(\mathrm{C})$ of a sector element (Balzek, 2001). The centroids of a surface element, denoted by a red $x$ on the figure, are integration points $(i p)$ and these are used in the evaluation of surface integrals. The continuity, momentum and energy equations (Equations 3.1, 3.2 and 3.3) are integrated over each finite control volume resulting in the following expressions: 


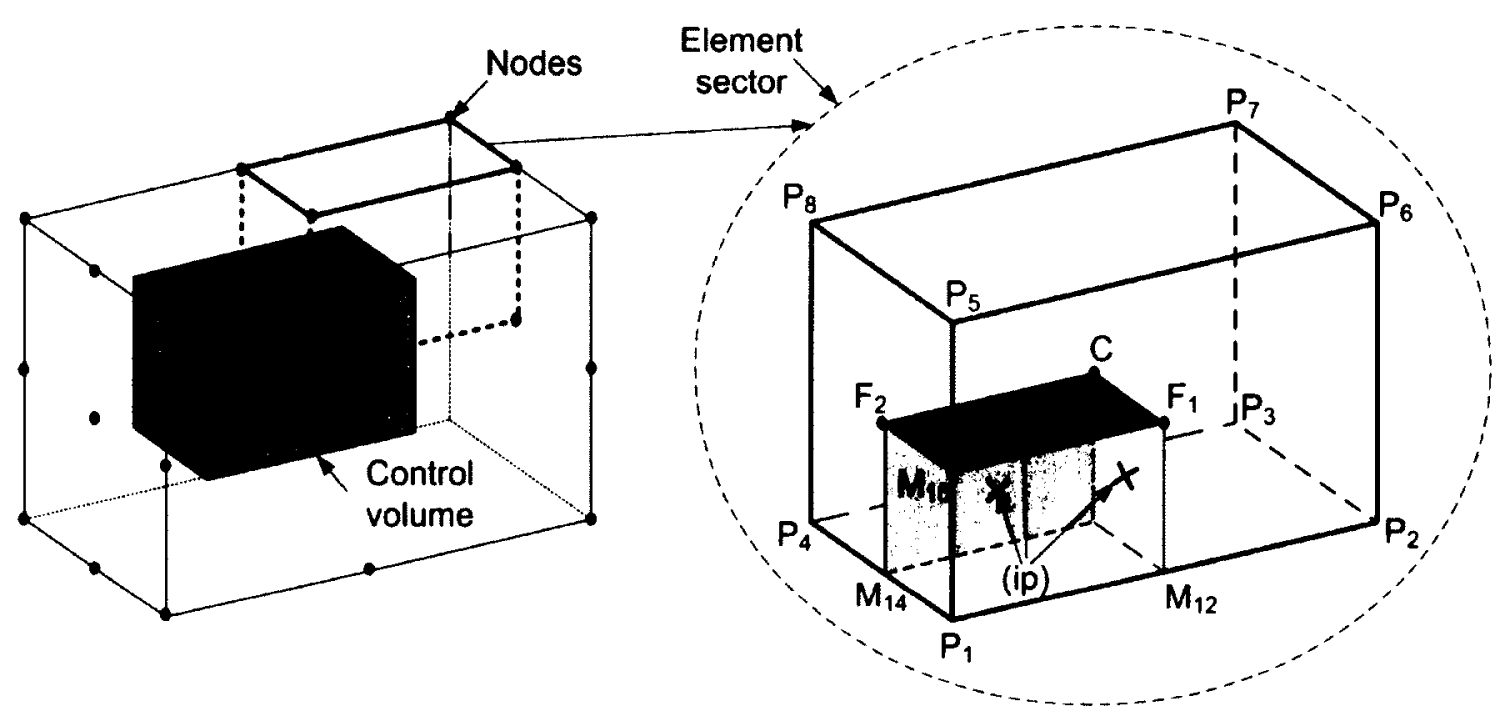

Figure 3.1: Setup of control volumes and elements in ANSYS CFX (adapted from Balzek, 2001).

$$
\begin{gathered}
\frac{\partial}{\partial t} \int_{Y} \rho d \mathscr{V}+\sum_{i p} \dot{m}_{i p}=0 \\
\frac{\partial}{\partial t} \int_{Y} \rho U_{i} d \mathscr{V}+\sum_{i p}\left(\dot{m}_{i p} U_{i}\right)_{i p}=-\sum_{i p}\left(P_{i p} \Delta n_{i}\right)_{i p}+\sum_{i p} \mu\left(\frac{\partial U_{i}}{\partial x_{j}}+\frac{\partial U_{j}}{\partial x_{i}}\right) \Delta n_{j} \\
\frac{\partial}{\partial t} \int_{y} \rho h_{0} d \mathscr{V}-\frac{\partial}{\partial t} \int_{y} P d \mathscr{V}+\sum_{i p}\left(\dot{m}_{i p} h_{0}\right)_{i p} \\
=\sum_{i p}\left(k \frac{\partial T}{\partial x_{i}} \Delta n_{i}\right)_{i p}+\sum_{i p} U_{i} \mu\left(\frac{\partial U_{i}}{\partial x_{j}}+\frac{\partial U_{j}}{\partial x_{i}}\right) \Delta n_{j}
\end{gathered}
$$

where $\mathscr{V}$ denotes the element volume, $\Delta t$ denotes a discrete timestep, and $\Delta n$ is the discrete outward-pointing normal unit vector. Volume integrals are evaluated over each three-dimensional sector surrounding a node and aggregated over all sector volumes pertaining to the control volume while surface integrals are evaluated at integration points $(i p)$. 
ANSYS CFX stores all flow data at the nodes. The variation in flow properties within each element is quantified using finite-element-based shape functions $\left(N_{i}\right)$. These shape functions are used as weighting functions applied on the quantities stored at the nodes of the element $\left(\phi_{i}\right)$ to obtain an approximation for the quantity at the integration points $\left(\phi_{i p}\right)$ located at the parametric local coordinates of the element $\left(r_{i p}, s_{i p}, t_{i p}\right)$.

$$
\phi_{i p}=\sum_{i=1}^{N_{\text {node }}} N_{i}\left(r_{i p}, s_{i p}, t_{i p}\right) \phi_{i}
$$

This discretization process simplifies to a second-order central-differencing scheme for hexahedral grids with orthogonal gridlines and a uniform node spacing.

The ANSYS CFX algorithm uses a collocated configuration where all properties are evaluated at the same point. In this approach, the mass flow term $\left(\dot{m}_{i p}=\rho U_{i} \Delta n_{j}\right)$ is coupled to the pressure through expressing the velocity at an integration point as the average of the surrounding nodal values adjusted by a redistribution of pressure gradients and previous timestep velocities. The details of this coupling algorithm are discussed in the ANSYS solver theory guide (ANSYS, 2007).

The viscous diffusion terms in Equation 3.7 and 3.8 are evaluated using the spatial derivatives of the shape functions:

$$
\left.\frac{\partial \phi_{i p}}{\partial x}\right|_{i p}=\left.\sum_{n} \frac{\partial N_{n}}{\partial x}\right|_{i p} \phi_{n}
$$

The temporal derivatives are is evaluated using a second order backwards Euler differencing scheme:

$$
\frac{\partial}{\partial t} \int_{Y^{\prime}} \rho U_{i} d \mathscr{V} \approx \mathscr{V} \frac{1}{\Delta t}\left(\frac{3}{2}\left(\rho U_{i}\right)-2\left(\rho U_{i}\right)^{n-1}+\frac{1}{2}\left(\rho U_{i}\right)^{n-2}\right)
$$

where $n-1$ and $n-2$ superscripts denote the first and second previous timesteps, respectively. 


\subsubsection{Solution Algorithm}

The linear system of equations obtained by evaluating Equations 3.6 and 3.7 over each integration point with linearization of the nonlinear terms in the resultant equations can be written in matrix form as shown in Equation 3.12.

$$
\sum_{n b_{i}} a_{i}^{n b} \phi_{i}^{n b}=b_{i}^{n b}
$$

where

$$
\begin{gathered}
a_{i}^{n b}=\left(\begin{array}{llll}
a_{u u} & a_{u v} & a_{u w} & a_{u p} \\
a_{v u} & a_{v v} & a_{v w} & a_{v p} \\
a_{w u} & a_{w v} & a_{w w} & a_{w p} \\
a_{p u} & a_{p v} & a_{p w} & a_{p p}
\end{array}\right)_{i} \\
\phi_{i}^{n b}=\left(\begin{array}{c}
U \\
V \\
W \\
P
\end{array}\right)_{i} \\
b_{i}^{n b}=\left(\begin{array}{c}
b_{U} \\
b_{V} \\
b_{W} \\
b_{P}
\end{array}\right)_{i}
\end{gathered}
$$

Here $i$ is a finite-volume index number, $n b$ indicates that the equation set up for node $i$ is dependent on the neighbouring nodes, and $a_{i}$ and $b_{i}$ are the equation coefficients and right-hand-side term, respectively. The energy conservation equation (Equation 3.8), which is a scalar equation, is solved by the ANSYS CFX solver following the coupled solution of the continuity and momentum equations (ANSYS, 2007).

The ANSYS CFX algorithm uses an iterative incomplete lower upper (ILU) sparse 
matrix inversion scheme to solve the system of equations in Equation 3.12 (ANSYS, 2009). The algorithm uses initial conditions to generate an approximate solution to Equation 3.12 which is then iterated through a series of inner loops which is embedded in a set of outer loops iterations between each timestep. The inner loop iteration solves the system of equations using an algebraic multi-grid algorithm consisting of restriction phases, where the equations are solved on progressively coarser grids to more-rapidly propagate large-wavelength errors through the computational domain, and then prolongation phases, where the solution is passed back up to the finer grids to reduce small-wavelength errors. The solver proceeds in a double $\mathrm{W}$ routine through the different levels of coarseness, as shown in Figure 3.2, where the integer in the circles represent the number of iterations performed at each grid level. The outer loop iterations are used to update the coefficients of Equation 3.12 until the error in the solution, which is referred to as the residual, falls below user-specified convergence criterion has been reached. The present study uses a criterion of maximum residual of $10^{-4}$, in the computational domain for any one of the governing equations which ensures that the root-mean-square (rms) residual is on the order of $10^{-6}$ which has

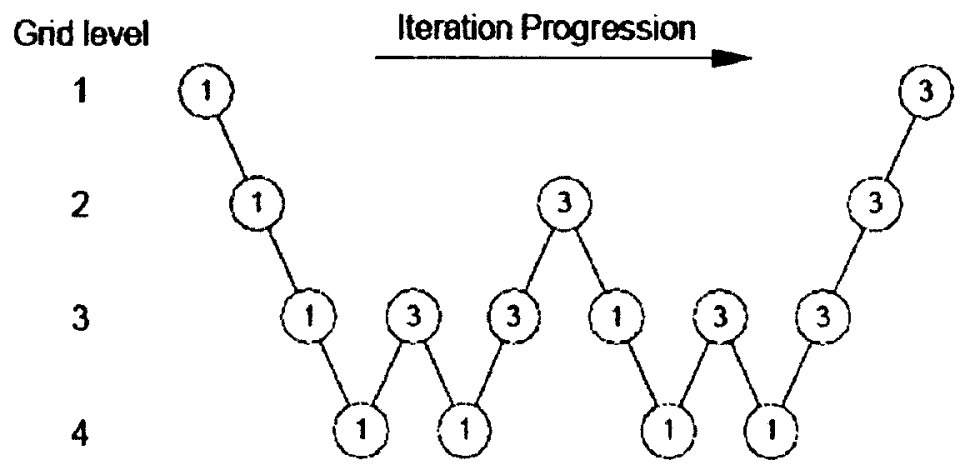

Figure 3.2: Schematic illustrating the W-cycle used as the inner iteration loop by the multigrid scheme in ANSYS CFX. Integers refer to the number of solutions performed at each grid level. Reproduced from McAuliffe (2007). 
been shown to be suitable for numerical simulation of transition and turbulence (e.g. McAuliffe and Yaras, 2007; Brinkerhoff and Yaras, 2011). Based on these criteria, simulations achieve convergence within 4 outer loop iterations.

\subsection{Computational Setup}

\subsubsection{Laminar Planar Shear Layer in Proximity to a Wall}

\subsubsection{Domain Geometry}

The two-dimensional computational domain, shown schematically in Figure 3.3, consists of a $300 \mathrm{~mm}$ long, no-slip, infinitesimally thin splitter plate located between domains 1 and 2 at a specified height above a flat, no-slip wall. Inflow boundaries consisting of spatially-uniform and time-invariant streamwise velocities $U_{1}$ and $U_{2}$ are specified at the inlets above and below the splitter plate, respectively, and a boundary layer develops on both surfaces of the plate. The length of the splitter plate is sized for each test case to satisfy the following criteria: (a) be sufficiently long to enable the development of viscous instability in the high-momentum-stream splitterplate boundary layer; and (b) ensure that the trailing-edge momentum thickness of the high-momentum-stream boundary layer remains constant for all test cases. The latter requirement allows the Reynolds number of the free shear layer to be varied independently of the momentum thickness of the shear layer at the splitter-plate trailing edge. In the lower Reynolds number cases, this requires that the plate length be reduced to $100 \mathrm{~mm}$. At the splitter plate trailing edge, the boundary-layer flows separated by the plate are merged to form a shear layer that is allowed to develop within a mixing region of length $L=600 \mathrm{~mm}$. The length of the mixing region is sized conservatively to capture the initial free-shear-layer development that is expected to be two-dimensional, namely the growth of perturbations in the shear layer through 


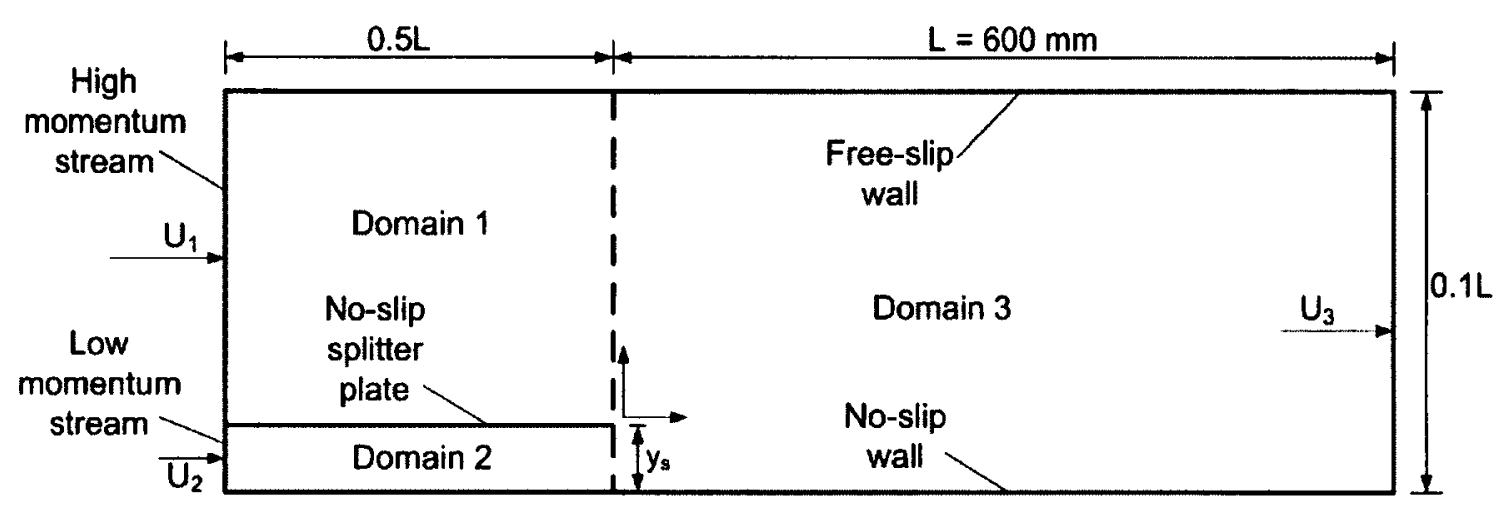

Figure 3.3: Schematic of the two-dimensional computational domain for the simulations on wall-proximity effects (not to scale).

the dominant Kelvin-Helmholtz instability mode, and roll-up of the shear layer into coherent spanwise vortices. A free-slip wall is specified along the upper wall of the computational domain, located $60 \mathrm{~mm}$ above the bottom, no-slip wall. The domain height is sized so that the vortices shed from the shear layer induce a velocity on the upper wall that is less than $1 \%$ of the inlet velocity specified for the high-momentum stream, thus minimizing the influence of the upper wall on the shear-layer development. The height of the splitter plate from the lower wall is varied systematically over the range $y / \theta_{s}=7.25-29.0$, where $\theta_{s}=0.41 \mathrm{~mm}$ is the momentum thickness of the high-momentum-stream boundary layer at the trailing edge of the splitter plate.

\subsubsection{Spatial Grid}

A structured grid of hexahedral elements is mapped to the computational domain, yielding a total node count of 460,000 to 580,000 depending on the proximity of the splitter plate to the lower wall and the flow Reynolds number. The node spacing in the streamwise and wall-normal directions are shown in Figure 3.4. Nodes are spaced in the streamwise direction to provide the finest spatial resolution near the trailing edge of the splitter plate and within the downstream mixing region. In this 


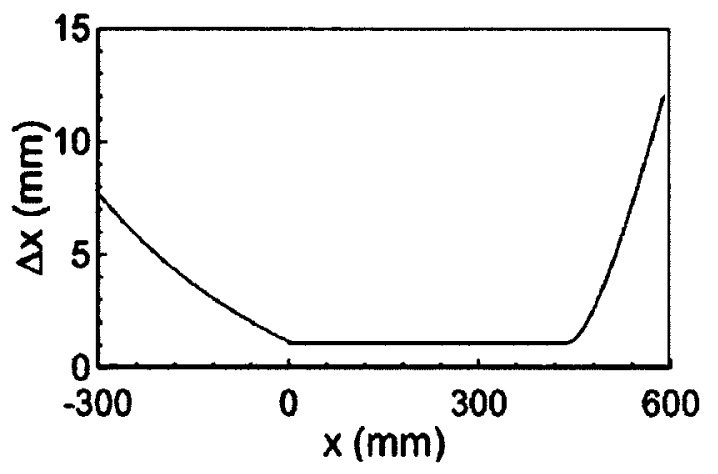

(a)

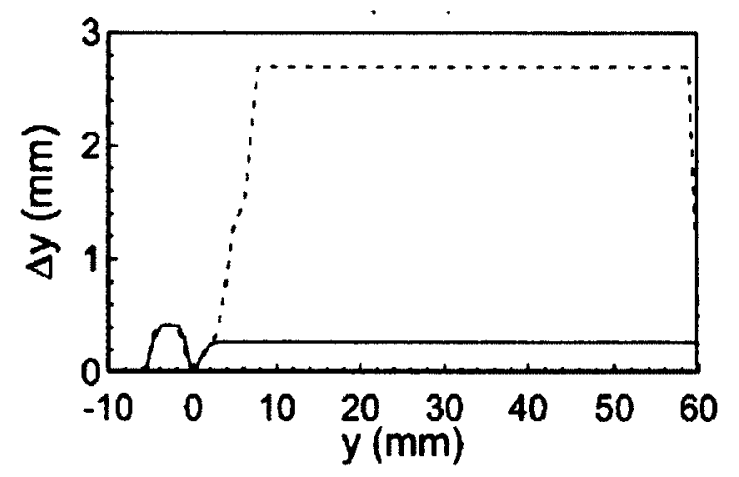

(b)

Figure 3.4: Node spacing in the (a) streamwise and (b) wall-normal directions (solid line: mixing region; dashed line: splitter plate region).

region, the streamwise node spacing is $\Delta x=1.06 \mathrm{~mm}$. Expressed in wall units based on the friction velocity in the high-momentum stream at the splitter-plate trailing edge, this spacing corresponds to $\Delta x^{+}=17$. Beyond $x=450 \mathrm{~mm}$, the streamwise node spacing is increased to place the outflow boundary sufficiently far away from the region of interest, as the outflow boundary treatment does not allow convective flow structures to exit the domain without being artificially distorted. This artificial effect on the flow-field by the outflow boundary is confirmed to be limited to within $0.05 \mathrm{~L}$ streamwise length upstream of the outflow boundary.

The node spacing in the wall-normal direction is given in Figure $3.4 \mathrm{~b}$. In the high-momentum stream, the wall-normal node spacing is $\Delta y=0.033 \mathrm{~mm}$ at the wall $\left(\Delta y^{+}=0.53\right.$ based on the friction velocity in the high-momentum stream at the splitter-plate trailing edge), which is gradually increased to a value of $1.06 \mathrm{~mm}$ $\left(\Delta y^{+}=17\right)$ at the boundary-layer edge and maintained to the top free-slip boundary of the computational domain. In the low-momentum stream, the wall-normal node spacing is $\Delta y=0.033 \mathrm{~mm}\left(\Delta y^{+}=0.53\right)$ at the no-slip wall at the bottom of the computational domain and at the splitter-plate surface, and gradually increases to $\Delta y=0.400 \mathrm{~mm}$ in the core region of the low-momentum stream. In the mixing 
Table 3.1: Node spacings used in DNS studies involving separation bubbles.

\begin{tabular}{lcccc}
\hline Study & $\Delta x^{+}$ & $\Delta y_{\text {wall }}^{+}$ & $\Delta y_{\max }^{+}$ & $\Delta z^{+}$ \\
\hline Present Study & 17 & 0.5 & 4.3 & - \\
McAuliffe and Yaras (2007) - Low Turbulence & 24 & 0.6 & - & 24 \\
McAuliffe and Yaras (2007) - High Turbulence & 24 & 0.5 & - & 24 \\
Na and Moin (1988) & 18 & 0.11 & 22.7 & 11 \\
Alam and Sandham (2000) & 14,20 & $<0.5$ & 0.9 & 6 \\
Michelassi et al. (2002) & 10 & 0.8 & - & 3 \\
Kalitzin et al. (2003) & 28 & - & 2.3 & 19 \\
\hline
\end{tabular}

region downstream of the splitter-plate trailing edge, a uniform y-direction spacing of $0.26 \mathrm{~mm}\left(\Delta y^{+}=4.3\right)$ is maintained throughout the mixing region, except for a more-finely resolved region at the wall-normal height corresponding to the height of the splitter plate. Table 3.1 compares the spatial resolution of several other simulations of separated laminar shear layers indicating favourable agreement with the present study. These grid-node spacings are known to provide satisfactory resolution as demonstrated by McAuliffe and Yaras (2007), Na and Moin (1998), Michelassi et al. (2002) and Kalitzin (2003), amongst others. To accommodate two-dimensional grids in ANSYS CFX, which operates by discretizing the domain into three-dimensional finite volumes, the computational grids use 5 nodes in the spanwise direction with a total spanwise length of $2.8 \mathrm{~mm}$. The spanwise length was verified to be sufficiently small so that there is no influence of any three-dimensional effects on the development of the two-dimensional flow field. The interfaces between the three domains of the computational domain are transparent to fluid flow and connected by a general grid interface (GGI) wherein the solver interpolates the solution between the upstream and downstream domains. Coincidence of the nodes at the interface between the mating domains is maintained to eliminate the artificial modification of flow gradients that are observed to otherwise occur during the interpolation. 


\subsubsection{Time-Step Size and Simulation Duration}

Based on previous studies by McAulliffe and Yaras (2007) a time-step size of $150 \mu s$ is used in all simulations, which corresponds to a Courant number of 1.6. Domain 1 and 3 are initialized to the high momentum velocity while domain 2 is initialized to the low momentum velocity. Using these initial conditions, the simulation is advanced in time to a statistically-steady state. Statistical convergence of the simulation is determined by assessing the standard deviation of the vortex-shedding frequency as the simulation progressed in time. A region of flow conservatively sized to enclose a primary Kelvin-Helmholtz $(\mathrm{K}-\mathrm{H})$ vortex is placed one K-H wavelength beyond the site of roll-up. Circulation within the enclosed region reaches a maximum when a vortex is completely enclosed by the region. A sample time trace, shown in Figure 3.5 , illustrates that about 20,000 timesteps are needed to reach a statistically-steady state. Following the attainment of statistically-steady state, the simulation is further advanced for approximately 2100 timesteps to obtain data for flow analysis. The simulations are partitioned and simulated in parallel on 4 Intel Core i5 processors using the message-passing interface (MPI) parallelization algorithm. The CPU time for each simulation is $170 h$.

\subsubsection{Test Matrix}

Table 3.2 shows the test matrix. The independently varied parameters are the momentum-thickness Reynolds number of the high-momentum stream at the splitterplate trailing edge $R e_{\theta s}$, the velocity-difference ratio, $R$, and the wall proximity, $y_{s}$. The velocity-difference ratio, defined as $R=2\left(U_{1}-U_{2}\right) /\left(U_{1}+U_{2}\right)$, is used to characterize the difference in velocity between the high- and low-momentum streams. The proximity of the splitter plate to the no-slip lower wall is normalized by the momentum thickness of the high-momentum stream boundary layer at the splitter-plate 


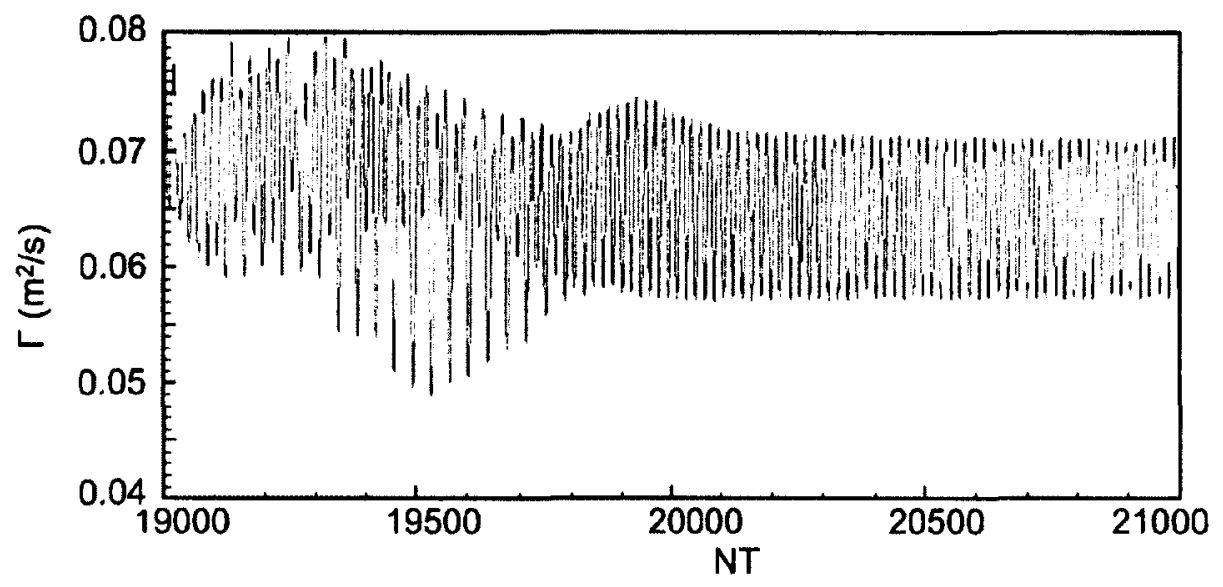

Figure 3.5: Temporal variation of circulation calculated within a region near the trailing edge of the splitter plate.

trailing edge.

The range of wall proximities, Reynolds numbers, and velocity difference ratios are chosen to capture the typical ranges observed in studies of separation bubbles and free shear layers in published literature (e.g. McAuliffe and Yaras, 2007; Rist and Augustin, 2006; Watmuff, 1999). The static pressure in the domain is dictated by the outflow plane static pressure which is set to a value of $101.3 \mathrm{kPa}$ that is kept constant at the outflow plane in an area-averaged sense. The density of the working fluid, which is air, is kept at a constant value of $1.22 \mathrm{~kg} / \mathrm{m}^{3}$. The simulations are thus performed in an incompressible state with only the continuity and momentum equations used to compute the flow field. 
Table 3.2: Flow and geometry parameters for the simulations of 2D laminar free-shear-layer development in wall proximity.

\begin{tabular}{llllll}
\hline Case & $R_{\theta_{s}}$ & $U_{1}(\mathrm{~m} / \mathrm{s})$ & $U_{2}(\mathrm{~m} / \mathrm{s})$ & $\mathrm{R}$ & $y_{s} / \theta_{s}$ \\
\hline \hline 1 & 320 & 11.8 & 0.366 & 1.88 & 7.25 \\
2 & 320 & 11.8 & 0.366 & 1.88 & 10.8 \\
3 & 320 & 11.8 & 0.366 & 1.88 & 14.5 \\
4 & 320 & 11.8 & 0.366 & 1.88 & 29.0 \\
5 & 108 & 3.94 & 0.122 & 1.88 & 7.25 \\
6 & 108 & 3.94 & 0.122 & 1.88 & 10.8 \\
7 & 108 & 3.94 & 0.122 & 1.88 & 14.5 \\
8 & 108 & 3.94 & 0.122 & 1.88 & 29.0 \\
9 & 320 & 11.8 & 3.33 & 1.22 & 7.25 \\
10 & 320 & 11.8 & 3.33 & 1.22 & 10.8 \\
11 & 320 & 11.8 & 3.33 & 1.22 & 14.5 \\
12 & 320 & 11.8 & 3.33 & 1.22 & 29.0 \\
\hline
\end{tabular}

\subsubsection{Laminar Planar Free-Shear Layer with Thermody- namic Property Gradients}

\subsubsection{Domain Geometry}

The three-dimensional domain consists of an entry region, splitter-plate region and free shear layer region, shown schematically in Figure 3.6. All dimensions are presented in terms of the splitter plate length $(L=300 \mathrm{~mm})$. An entry region with a free-slip surface between the low- and high-momentum streams is provided upstream of the splitter plate so that the inflow stream can gradually flow onto the splitter plate, without creating artificially-high shear that would otherwise be created if the computational domain inlet with plug velocity profiles were specified at the splitter-plate leading edge. Furthermore, the use of a free-slip surface between the two streams ensured absence of energy and momentum transfer between the two streams upstream of the splitter plate. The splitter-plate domain consists of an upper and a lower sub-domain each with streamwise, plate-normal and spanwise dimensions of $L$, 


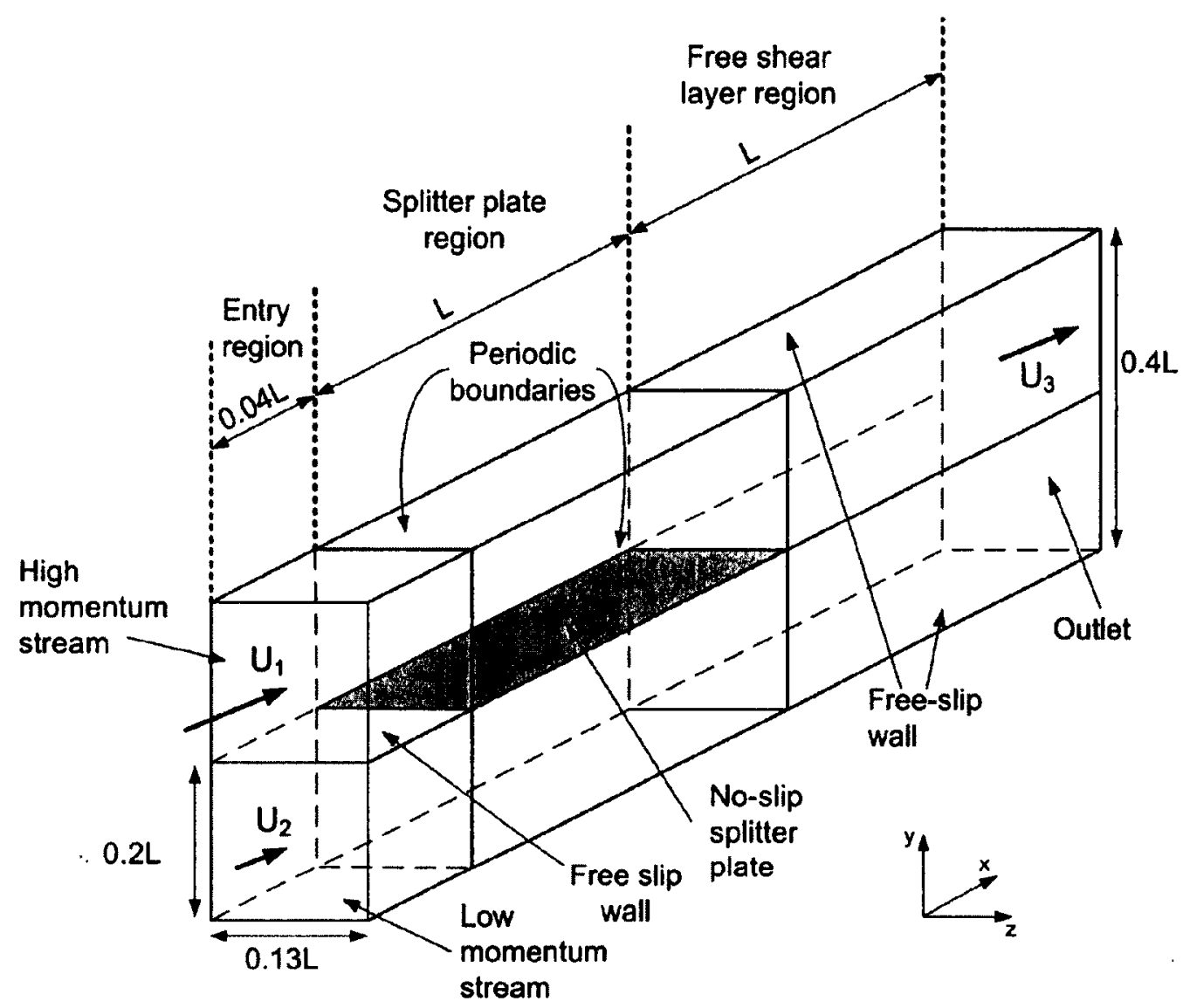

Figure 3.6: Schematic of the computational domain for the laminar free shear-layer simulations with spatial gradients in supercritical thermophysical properties (not to scale).

$0.2 L$ and $0.13 L$ respectively. A no-slip, infinitesimally-thin, splitter plate of sufficient length to facilitate flow Reynolds number beyond the critical Reynolds number on the high-speed-stream side is specified at the interface between the upper and lower domain of this region. This setup allows for the study of the interaction between the splitter-plate boundary layer instability and the free-shear-layer instability. The free-shear-layer region has dimensions of $L, 0.2 L$ and $0.13 L$ in the streamwise, spanwise and plate-normal directions, respectively. The streamwise length of this region is selected to provide sufficient length for the primary mode of instability to develop. 
The spanwise width of the domain is sized to allow for unrestricted development of spanwise nonuniformities in the form of evenly-spaced streamwise vortices with a wavelength of $\lambda_{z}^{+}=100$ as per the perturbation theory of Baig and Chernyshenkov (2004). The $0.65 \mathrm{~L}$ width of the region of fine spanwise resolution should allow for up to eight such vortices to develop in this resolved region. The domain height of $0.4 L$ places the upper and lower domain boundaries sufficiently far from the shear layer to allow for unrestricted deformation of the shear layer under the influence of the dominant instability mechanisms. The velocity perturbations at the upper and lower walls of the domain remained within $2 \%$ of the high-momentum-stream velocity $\left(U_{1}\right)$.

\subsubsection{Spatial Grid}

The streamwise, spanwise and plate-normal distributions of nodes are shown in Figure 3.7. A total of 16 million nodes are used in the computational domain distributed in a manner to produce a grid of hexahedral elements. In the splitter plate domain, the streamwise node spacing is relatively large $(\Delta x>7 \mathrm{~mm})$ upstream of the expected location of the critical Reynolds number $(x=350 \mathrm{~mm}$ in Figure $3.7 \mathrm{a})$ and is refined downstream of this position to $\Delta x=1.04 \mathrm{~mm}\left(\Delta x^{+}=22.8\right.$, expressed in wallunits based on the viscosity and friction velocity of the supercritical water flow in the high-speed stream at the splitter-plate trailing edge) such that the streamwise evolution of Tollmien-Schlichting instability in the splitter-plate boundary layer is adequately resolved. The region immediately downstream of the splitter-plate trailing edge (400 mm $<x<540 \mathrm{~mm}$ on Figure 3.7a) contains the most refined grid to capture the development of instability and transition in the free shear layer. The minimum values of $\Delta x, \Delta y$ and $\Delta z$ in this region correspond to normalized values of $\Delta x^{+}=11.4, \Delta y^{+}=0.53$, and $\Delta z^{+}=5$ based on the viscosity and friction velocity in the high-speed stream at the splitter-plate trailing edge. Toward the end of the freeshear-layer region, at a distance $500 \mathrm{~mm}$ from the outlet, the grid spacing is gradually 


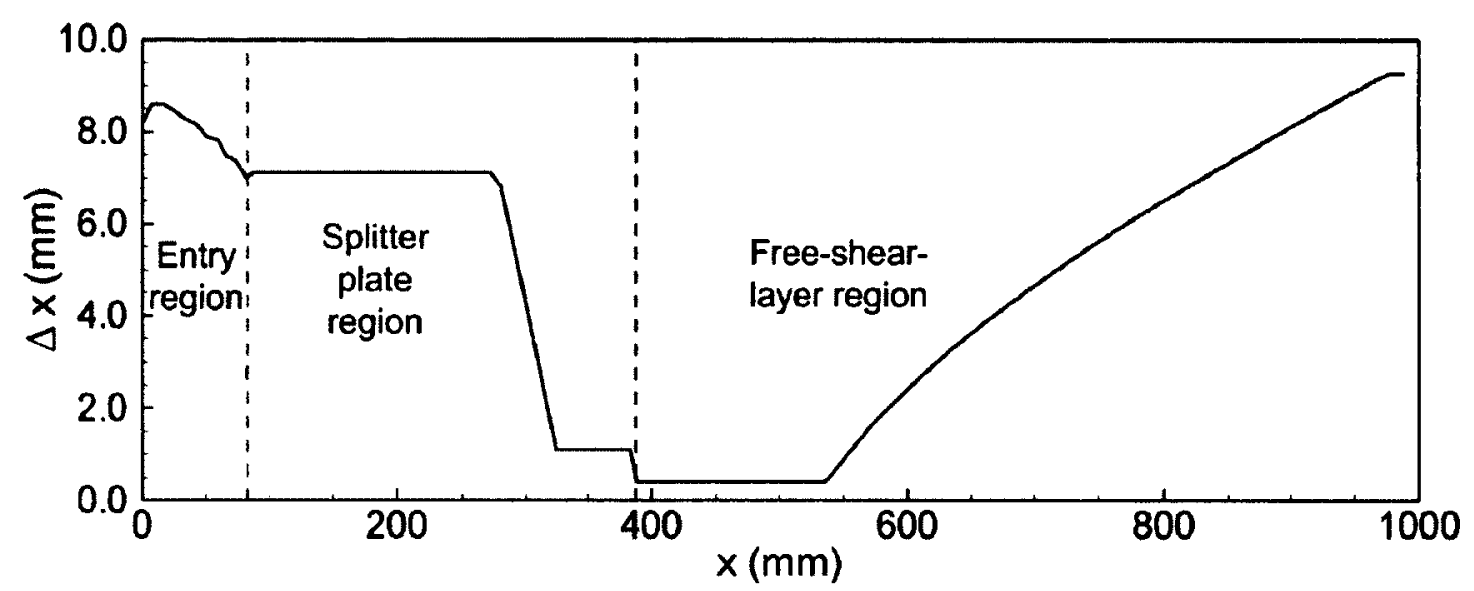

(a)

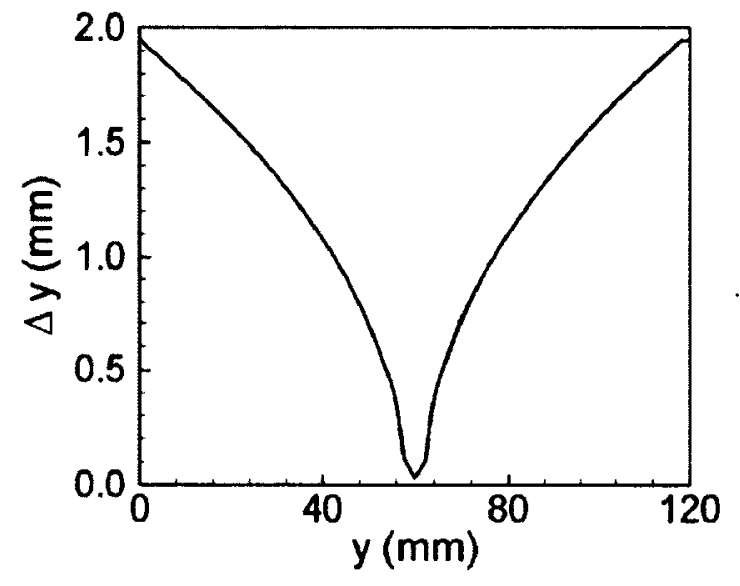

(b)

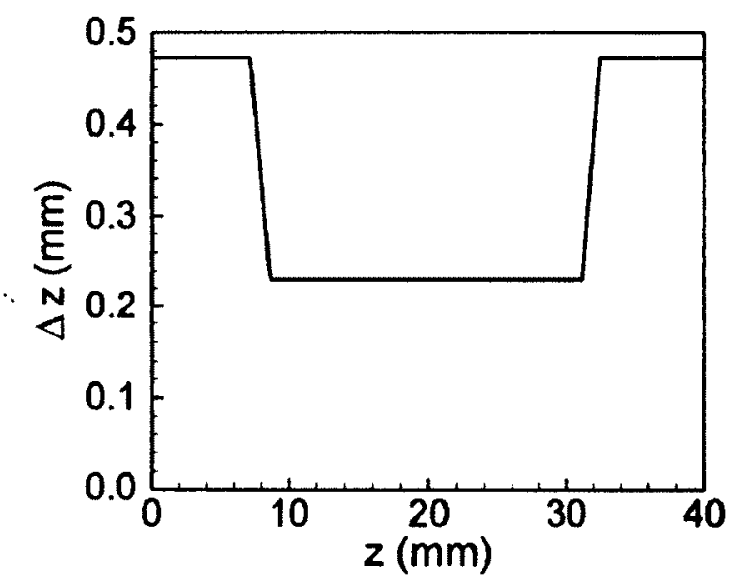

(c)

Figure 3.7: Node spacing in the (a) streamwise, (b) plate-normal (c) and spanwise directions.

coarsened to serve as a "buffer length" that minimizes the artificial influence the outlet has on the upstream flow.

\subsubsection{Time-Step Size and Simulation Duration}

The simulation of the free-shear layer development in air is performed with a timestep size of $150 \mu s$ which corresponds to a Courant number $(\Delta t U / \Delta x)$ value of 1.6. Several studies (e.g. Friedrich et al., 2001; Azih et al., 2012) suggest that in turbulent, 
wall-bounded flows of supercritical fluids, the timestep must be less than $0.2 \nu / u_{\tau}^{2}$ to ensure numerical stability. Therefore, a timestep size of $200 \mu s$, which is conservatively below this criterion $\left(0.2 \nu / u_{\tau}^{2}=260 \mu s\right)$, corresponding to a Courant number of 0.01 is adopted for the supercritical water simulations. The simulations are run to statistically-steady state wherein the variation in properties diminishes in a statistical sense. The free-shear-layer simulation with air is advanced for 3,000 timesteps until statistically-steady state is reached, after which 2,048 timesteps of data are collected for analysis. A total CPU time of $2160 h$ is required for the completion of this simulation. The free-shear-layer simulation with supercritical water is advanced for 10,000 timesteps until statistically-steady state is reached, after which 6,000 timesteps of data are collected for analysis. A total CPU time of $5760 h$ is required for the completion this simulation. These simulations are performed on 6 Intel Xeon L5140 processors with 4 cores each for a total of 24 cores. The computation domain is partitioned using the multilevel graph partitioning software (MeTiS).

\subsubsection{Test Matrix}

The test matrix is provided in Table 3.3. For the simulation with air as the working fluid, top-hat velocity profiles with $U_{1}=11.7 \mathrm{~m} / \mathrm{s}$ and $U_{2}=2.7 \mathrm{~m} / \mathrm{s}$ are specified at the inlet of the high- and low-momentum streams, respectively. The simulation with supercritical water as the working fluid is specified with inlet velocities of $U_{1}=0.083 \mathrm{~m} / \mathrm{s}$ and $U_{2}=0.0026 \mathrm{~m} / \mathrm{s}$ to keep dynamic similarity in Reynolds number and velocity difference ratio with the simulation using air. For the simulation with supercritical water, a cross-stream temperature gradient is created by specifying a high-momentum stream temperature of $T_{1}=630 \mathrm{~K}$ and a low-momentum stream temperature of $T_{2}=670 \mathrm{~K}$ with a no-slip splitter-plate surface temperature of $T_{w}=654 \mathrm{~K}$ corresponding to the pseudocritical temperature of water at $24 \mathrm{MPa}$. 
This temperature range corresponds to conditions expected for shear layers in supercritical water-cooled nuclear reactors (SCWR) while maintaining the highest property variations near the inflection point of the free-shear-layer velocity profile. The static pressure at the outflow boundary is set to $P=24 M P a$, which is kept constant in an area-averaged sense.

Table 3.3: Test matrix for the subcritical single-phase and supercritical laminar- free shear layer simulations.

\begin{tabular}{llllllllll}
\hline Case & Fluid & $T_{1}(K)$ & $T_{2}(K)$ & $T_{w}(K)$ & $P($ bar $)$ & $\operatorname{Re}_{\theta_{s}}$ & $U_{1}(\mathrm{~m} / \mathrm{s})$ & $U_{2}(\mathrm{~m} / \mathrm{s})$ & $R$ \\
\hline 1 & Air & 300 & 300 & 300 & 1.013 & 320 & 11.8 & 2.7 & 1.25 \\
2 & Water & 630 & 670 & 654 & 240 & 320 & 0.108 & 0.0262 & 1.25 \\
\hline
\end{tabular}

\subsection{Potential Flow Analysis Methods}

\subsubsection{Steady Potential Flow Analysis}

A fully-developed shear layer profile is often modeled as a hyperbolic-tangent velocity profile superimposed on a uniform base flow where the hyperbolic-tangent component is responsible for the vorticity in the shear-layer (Zayernouri and Metzger, 2011). Following this framework, an inviscid model is developed by superimposing point vortices on a uniform base flow to model the velocity field at selected temporal instances of the shear-layer roll-up process.

$$
V_{t o t}=V_{b}+V_{i}
$$

where,

$$
\boldsymbol{V}=(U, V)
$$




\section{$\Delta x$}

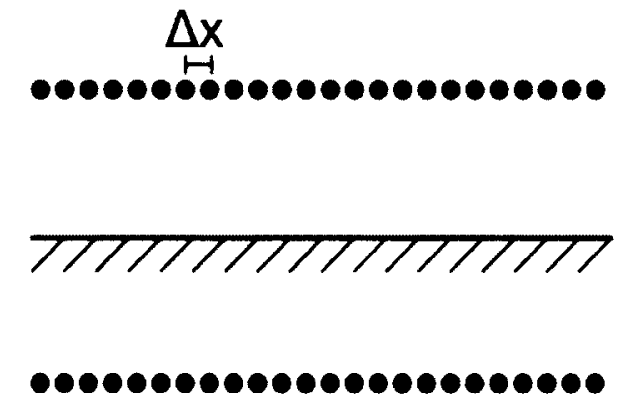

(a)

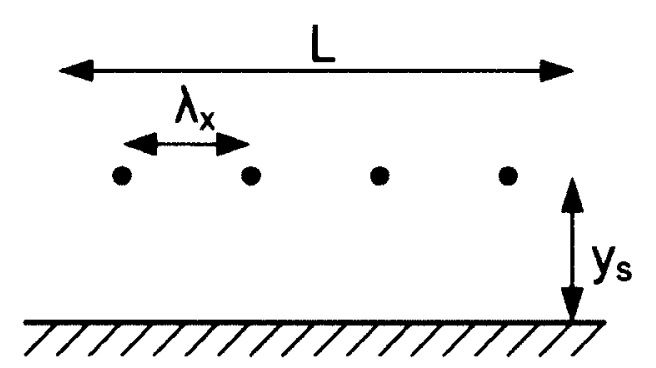

(c)

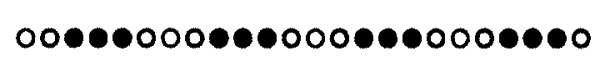

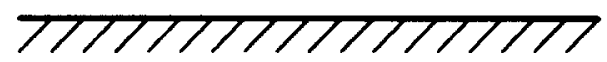

000000000000000000000000

(b)
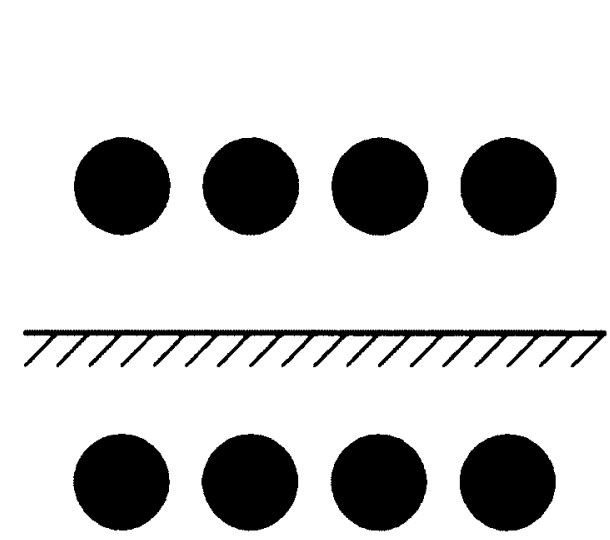

(d)

Figure 3.8: Vortex patterns for steady potential flow studies for (a) flat vortex sheet (b) streamwise-wavy vortex sheet (c) point vortices and (d) Rankine vortices (blue: negative vorticity; red: positive vorticity; line shows the position of the wall).

In this model of the shear-layer, $\boldsymbol{V}_{\boldsymbol{b}}$ is the velocity vector of the base flow, $\boldsymbol{V}_{\boldsymbol{i}}$ is the velocity vector induced by the point vortices and $\boldsymbol{V}_{\boldsymbol{t} \text { tot }}$ is the resultant velocity vector. The uniform base flow consists of streamwise velocity $U_{b}=6 \mathrm{~m} / \mathrm{s}$, which corresponds to the mean velocity near the vicinity of the shear layer for cases with $R e_{\theta s}=320$ and $R=1.88$ in the numerical study of the two-dimensional free shear layer as described in Sections 3.3.1.4.

As discussed in Chapter 2, shear-layer roll-up can be subdivided into the following stages: (a) an initially flat vortex sheet unperturbed by instabilities; (b) a streamwise wavy- wavy in the sense of streamwise period variations in the vorticity magnitude 
-vortex sheet created by the development of unstable K-H waves on the vortex sheet; (c) and a fully rolled-up discrete vortex. Each of these stages are modeled separately by superimposing a uniform flow with a series of point vortices spatially distributed to approximate one of the three stated stages of the shear layer development, as shown schematically in Figure 3.8. The flat shear layer is modeled by a finite number of point vortices distributed along a straight line as shown in Figure 3.8a. The streamwise wavy shear layer is modeled by a finite number of point vortices of varying vorticity magnitude, as shown in Figure $3.8 \mathrm{~b}$ where vorticity strength is represented by the degree of shading of the vortex element. Finally, the rolled-up shear layer state is modeled alternatively as a series of spatially-separated point vortices as shown in Figure 3.8c, and as a series of spatially separated Rankine vortices (vortices of finite core size with solid body rotation in the core) as shown in Figure 3.8d.

The vorticity distribution for each of these four cases are expressed as per Equations 3.18 to 3.20 :

Flat shear layer:

$$
\Gamma(x)=\frac{\Gamma_{t o t}}{L} \Delta x
$$

Streamwise-wavy shear layer:

$$
\Gamma(x)=2 \frac{\Gamma_{t o t}}{L}\left(\sin \left(\frac{x}{2 \pi \lambda_{x}}\right)+1\right) \Delta x
$$

Point-vortex and Rankine-vortex:

$$
\Gamma(x)= \begin{cases}\frac{\Gamma_{\text {tot }}}{L} \lambda_{x} & \text { if } x=n \lambda_{x} \\ 0 & \text { if } x \neq n \lambda_{x}\end{cases}
$$

where $\Gamma_{t o t}$ represents the total circulation in the vorticity distributions over the length of the shear layer considered, $\lambda_{x}$ represents streamwise wavelength of an unstable K-H 
wave, and $L$ is the length of the shear layer. These parameters are set to the values of $0.28 \mathrm{~m}^{2} / \mathrm{s}, 8 \mathrm{~mm}$ and $32 \mathrm{~mm}$, respectively, to ensure that for the point- and Rankine-vortex scenarios there are four vortices in the simulation domain, each with a circulation of $0.07 \mathrm{~m}^{2} / \mathrm{s}$. These conditions correspond to the conditions observed in the present Navier-Stokes simulations of the case with $R e_{\theta s}=320$ and $R=1.88$. The flat shear layer and streamwise-wavy shear layer each uses an array of point vortices with streamwise spacing, $\Delta x$, corresponding to a value of $0.32 \mathrm{~mm}$, which is determined based on a series of sensitivity studies to this spacing. The wall proximity $\left(y_{s}\right)$ is systematically varied from $3.5 \mathrm{~mm}-12.0 \mathrm{~mm}$, consistent with the corresponding Navier-Stokes simulations. The method-of-images is used to model the presence of the wall in an inviscid sense. Implementation of the method of images consists of placing an identical pattern of vortical elements with vorticity of the opposite sense of rotation an equal distance below the wall to be simulated.

The velocity induced by an element of the vortex-pattern for the vortex-sheet, streamwise-wavy sheet, and point-vortex patterns is given below:

$$
u_{\theta}=\frac{\Gamma}{2 \pi r}
$$

However, a Rankine-vortex behaves as a point-vortex away from the vortex core boundary and solid-body undergoing rotation within the boundary:

$$
u_{\theta}= \begin{cases}\frac{\Gamma r}{2 \pi r_{0}^{2}} & \text { if } r \leq r_{0} \\ \frac{\Gamma}{2 \pi r} & \text { if } r>r_{0}\end{cases}
$$

The vortex core radius of $r_{0}=2.5 \mathrm{~mm}$ corresponds to the radius of a rolled-up K-H vortex obtained through Navier-Stokes simulations with $R e_{\theta s}=320$ and $R=1.88$. 


\subsubsection{Transient Potential Flow Analysis}

The transient-potential-flow model consists of a perturbed vortex-sheet arrangement in close proximity to a wall. A vortex sheet is initialized as a horizontal line of point vortices $\left[\boldsymbol{r}_{\mathbf{1}}\left(0, y_{s}\right), \ldots \boldsymbol{r}_{\boldsymbol{i}}\left(x_{i}, y_{s}\right), \ldots \boldsymbol{r}_{n}\left(L, y_{s}\right)\right]$, as shown in Figure 3.9. A total of 200 vortex elements that are initially evenly spaced are used in the model based on a series of sensitivity studies. A vortex sheet length of $32 \mathrm{~mm}$ is used to provide sufficient room for four rolled-up vortices to develop. As discussed later, a sinusoidal perturbation of wavelength $\lambda_{x}=8 \mathrm{~mm}$ is applied onto the sheet with a total circulation of $\Gamma=0.07 \mathrm{~m}^{2} / \mathrm{s}$ enclosed in one wavelength of the vortex sheet. The wavelength and circulation correspond to the K-H vortices obtained for cases with $R e_{\theta s}=320$ and $R=1.88$ in the Navier-Stokes simulations. Periodic boundary-conditions are used to simulate the effect of an infinitely long vortex-sheet:

$$
x_{i}= \begin{cases}x_{i}+L & \text { if } x_{i}<0 \\ x_{i} & \text { if } 0<x_{i}<L \\ x_{i}-L & \text { if } x_{i}>L\end{cases}
$$

The method of images is applied to model an impermeable boundary at a distance $y_{s}$ from the vortex sheet as shown in Figure 3.9. The induced velocity on a given point vortex (i) on the vortex sheet by all other point vortices of the sheet and its image (j) is calculated using the Biot-Savart law:

$$
V_{i}=\sum_{j=1, i \neq j}^{n} \hat{r}_{i j} \times \hat{n} \frac{\Gamma}{2 \pi\left|r_{i j}\right|}
$$

Here $\boldsymbol{r}_{\boldsymbol{i} j}$ and $\boldsymbol{V}_{\boldsymbol{i}}$ are the relative position vector and velocity vectors and $\hat{\boldsymbol{i}}_{\boldsymbol{i}}$ and $\hat{\boldsymbol{n}}$ are unit vectors in the direction of the relative position vector and in a direction normal to the 2D plane of the simulation, respectively. 


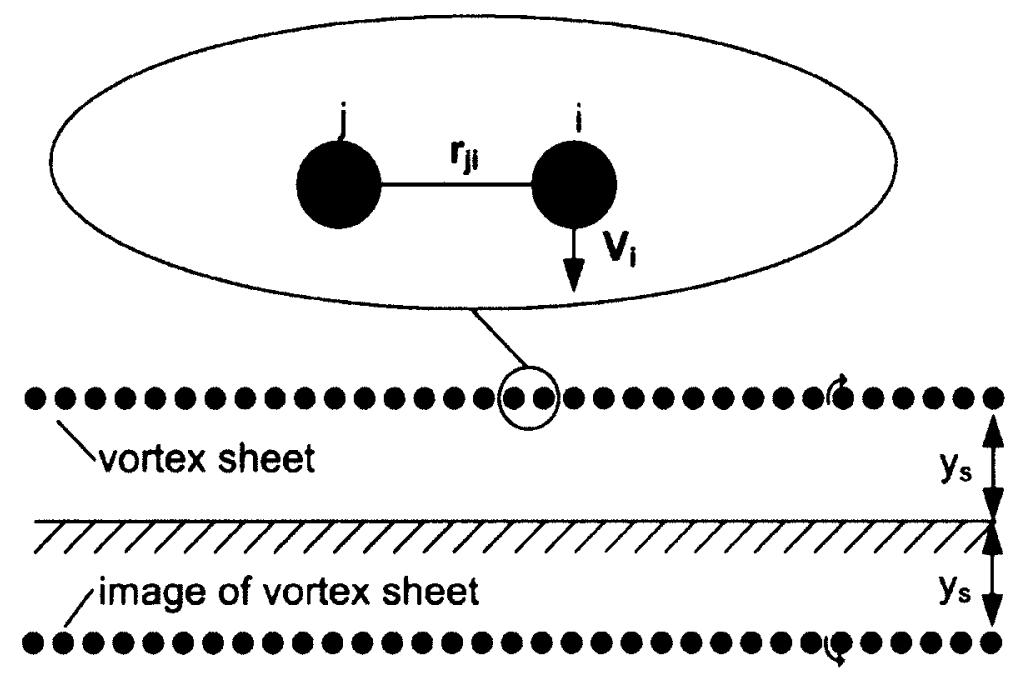

Figure 3.9: Schematic diagram of the initial setup of the transient potential flow vortex element model.

The induced velocity distribution calculated according to Equation 3.24 is superimposed onto a uniform base flow:

$$
V_{t o t}=V_{b}+V_{i}
$$

where $\boldsymbol{V}_{\boldsymbol{b}}$ is the velocity vector of the mean-flow and $\boldsymbol{V}_{\boldsymbol{t} \text { to }}$ is the resultant velocity vector. The uniform base flow consists of streamwise velocity $U_{b}=6 \mathrm{~m} / \mathrm{s}$ corresponding to the mean velocity near the vicinity of the shear layer for cases with $\operatorname{Re}_{\theta s}=320$ and $R=1.88$.

Time-varying solution of the deforming vortex sheet is determined by displacing the point vortices making up the vortex sheet and its image in small increments in time, with the amount and direction of displacement for each point vortex determined by the local velocity. As discussed in Chapter 2, the shear layer rolls-up at the frequency of the most-amplified perturbation. Therefore, the simulation is initialized by a small-sinusoidal perturbation of the form shown below to model $\mathrm{K}-\mathrm{H}$ waves that 
develop on the shear layer.

$$
\Delta y=y_{p} \sin \left(2 \pi x / \lambda_{x}\right)
$$

Here, $y_{p}=0.4 \mathrm{~mm}$ is the initial perturbation amplitude. The position of a vortexelement in the current timestep, indicated by superscript $n$, is based on the position at the previous timestep $n-1$ :

$$
r^{n}=r^{n-1}+V_{t o t} \Delta t
$$

where $r=(x, y)$ and the time step size is $\Delta t=300 \mu s$. The position of each point vortex at a given timestep is determined following the evaluation of the velocity at that timestep. This method of solving the position and velocity is essentially first-order since the present and preceding positions are used to evaluate the velocity. Consequently, the computational algorithm is expected to experience numerical diffusion. 


\section{Chapter 4}

\section{Effect of Wall Proximity on the Stability of Laminar Planar Free Shear Layers}

This chapter discusses the numerical results on the stability of a laminar planar shearlayer in close proximity to a wall in two-dimensional (2D) space. The chapter begins with a discussion on the stability characteristics of the splitter-plate boundary layer. Section 4.2 discusses the transient evolution of vortical structures, time-averaged and fluctuating values of the flow parameters, and the frequency of vortex-shedding in the free-shear layer developing downstream of the splitter-plate. Section 4.3 examines the effect of wall-proximity on the dominant frequency of vortex formation in the free-shear layer through a linear stability analysis. Section 4.4 and Section 4.5 interpret the free-shear-layer development obtained from the solution of the Navier-Stokes equations with the aid of transient and steady potential-flow models. 


\subsection{Instability Development in the Splitter-Plate Boundary Layer}

The discussion begins by analyzing the growth of perturbations in the splitter-plate boundary layers. The boundary layer on the low-momentum side of the splitter plate, which remains below the critical Reynolds number along the length of the splitterplate, does not involve any instabilities, and hence is not considered in this discussion. For brevity, the boundary layer of the high-momentum stream will hereafter be referred to as the splitter-plate boundary layer. A top-hat velocity profile is specified at the leading edge of the splitter-plate that evolves into a Blasius boundary layer profile as the flow develops along the length of the plate. The distributions of the displacement and momentum-thicknesses and of the shape factor are shown in Figure 4.1 for boundary-layer Reynolds numbers of $R e_{\theta s}=320$ and $R e_{\theta s}=108$, where $\theta_{s}$ denotes the momentum thickness of the high-momentum stream splitter-plate boundary layer at the trailing edge. A nearly uniform shape factor value of approximately 2.6 is maintained along the splitter-plate, which is consistent with the shape factor for the Blasius boundary layer. Imposing a top-hat velocity profile at the splitterplate leading edge may result in an unnatural development of the boundary layer in the immediate vicinity of the leading edge. However, as shown in Figure 4.1, the overall streamwise distributions of the computed displacement thickness, momentum thickness and shape factor compare well with the theoretical Blasius boundary layer. The deviation from the Blasius solution towards the trailing edge of the splitter plate is attributed to the upstream influence of the downstream instabilities that transmit into to the splitter-plate boundary layer through pressure fluctuations.

Linear stability theory predicts that beyond a critical Reynolds number, perturbations at unstable frequencies in the laminar boundary layer are amplified through the Tollmien-Schlichting (T-S) instability mode. Among others, experimental work 

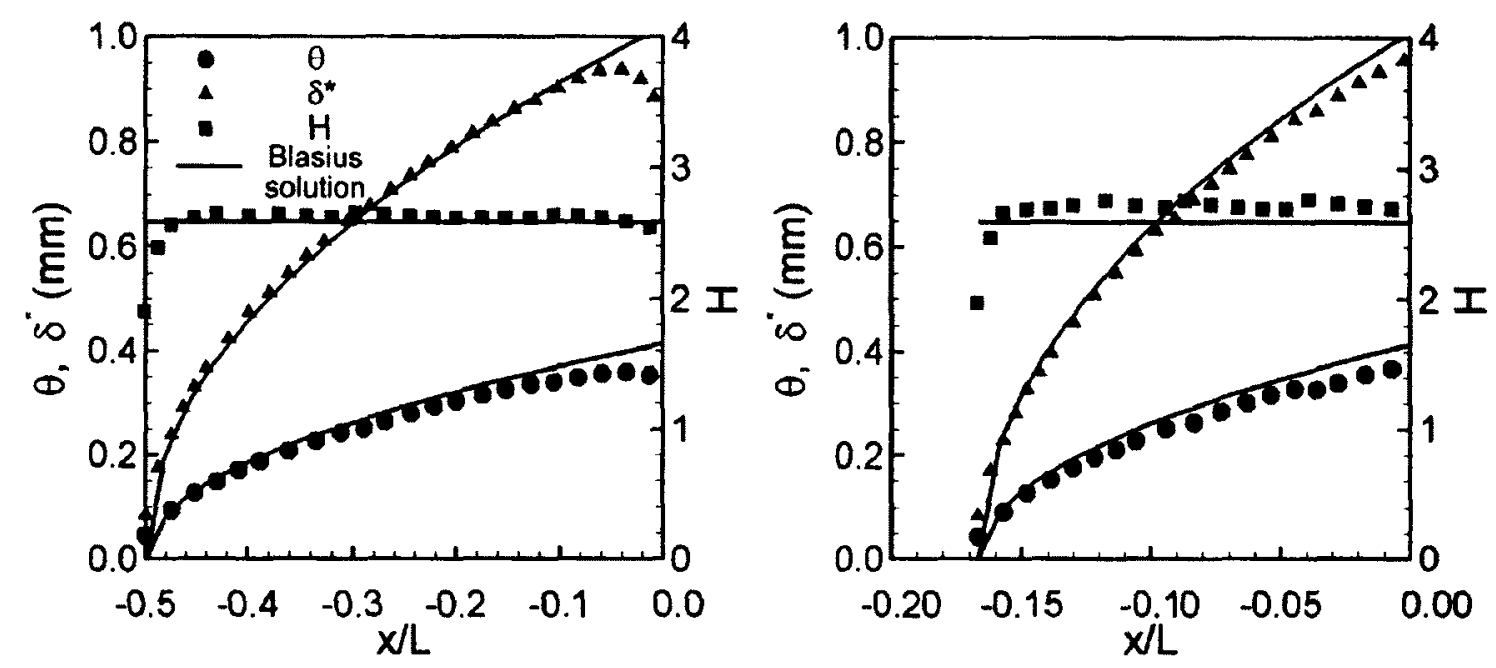

Figure 4.1: Displacement thickness, momentum thickness and shape factor growth in the splitter-plate boundary layer for (a) $\operatorname{Re}_{\theta \mathrm{s}}=320$ and (b) $\operatorname{Re}_{\theta \mathbf{s}}=108$.

by Reynolds and Saric (1986) and numerical work by Nayfeh and Reed (1986) suggest that T-S waves have a double-humped mode shape that manifests itself in the perturbation streamwise velocity profile, as discussed in Chapter 2. Figure 4.2 presents the mean velocity $(U)$ and perturbation velocity $(u)$ distributions across the thickness of the boundary layer at selected streamwise locations along the splitter-plate for $R e_{\theta s}=320$, where $U_{1}$ is the high-momentum freestream velocity and $u_{\max }$ is the local streamwise perturbation velocity maximum. Initially, the perturbation velocity distribution consists of numerical noise evidenced by the perturbation magnitude in the order of the floating-point precision of the calculations. However, as the T-S instability evolves with streamwise distance, a single-humped profile develops near the wall that is believed to be the manifestation of the critical layer. Following this, the mode shape develops into the standard double-humped profile observed by Reynolds and Saric (1986), Nayfeh and Reed (1986) and others.

In the present numerical simulations, the flow perturbations that are amplified in 


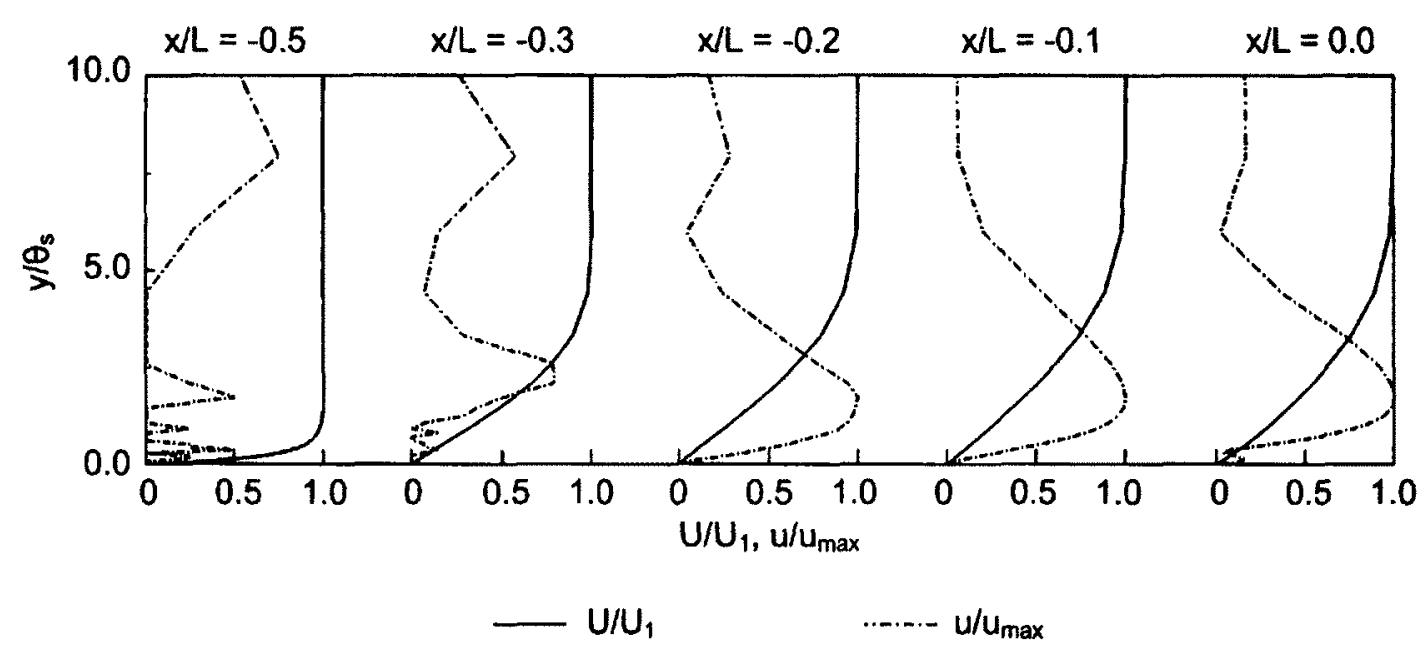

Figure 4.2: Development of the perturbation mode shapes in the splitter plate for $\mathbf{R e}_{\theta \mathbf{s}}=320$.

the unstable boundary layer are primarily associated with round-off errors, and consequently the initial perturbation levels are of the order of the floating-point precision of the calculations $\left(10^{-8}\right)$. Another source of perturbations is the downstream planar shear-layer, where pressure fluctuations due to the transient flow structures developing in the free-shear-layer travel as far as $20 \%$ of the splitter-plate length upstream of the trailing edge.

Figure 4.3 shows the amplification of perturbations within the splitter-plate boundary layer for two cases with the trailing-edge Reynolds number above the critical value $\left(\operatorname{Re}_{\theta s}=320\right)$ and one case with the Reynolds number below the critical value $\left(R e_{\theta s}=108\right)$. For $R e_{\theta s}=320$, low perturbation levels are observed in the boundary layer until $x / L=-0.24\left(R e_{\theta}=247\right)$, followed by exponential growth in perturbation amplitude from this location to the splitter-plate trailing edge. The computed streamwise location where the perturbations begin to amplify occurs farther downstream than the location of $x / L=-0.29\left(R e_{\theta}=200\right)$ predicted by linear stability theory (Schmid and Henningson, 2001). This shift may be due to the slight 


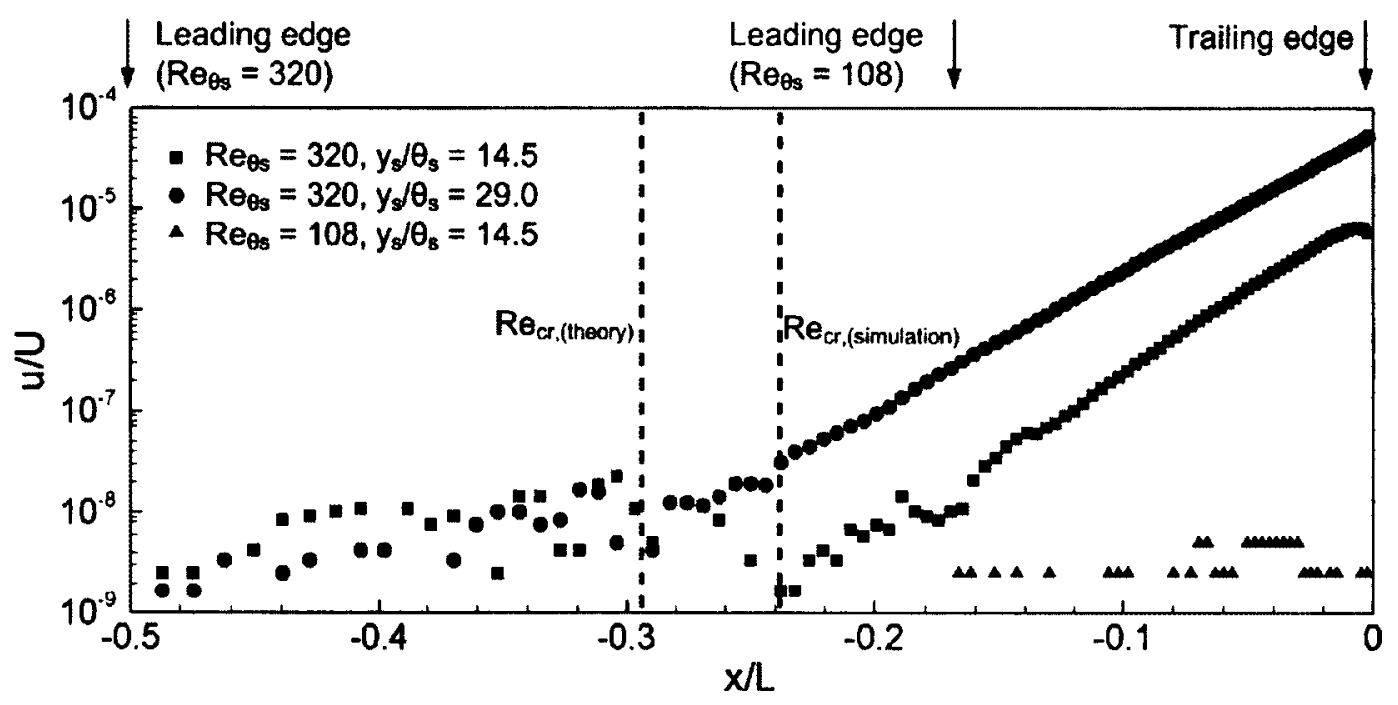

Figure 4.3: Streamwise growth of streamwise perturbation velocity magnitude in the splitter-plate boundary layer at $\mathrm{y} / \theta_{\mathrm{s}}=1$.

streamwise acceleration of the freestream caused by boundary-layer blockage. Despite the difference in the streamwise location where the onset of amplification occurs, the amplification rate of disturbances is similar in both $R e_{\theta s}=320$ cases. As expected, the case with $R e_{\theta s}=108$ shows essentially no amplification of perturbations by T-S instability along the length of the splitter plate.

The dominant frequency of the T-S waves is determined by analyzing the power spectra of the streamwise perturbation velocity at several streamwise locations in the splitter-plate boundary layer. The power spectra reveal amplification of several distinct frequencies, expressed as a Strouhal number $\left(S t_{\theta s}=f \theta_{s} / U_{1}\right)$. Most notably, peaks at $S t_{\theta s}=0.0064$ and 0.0125 are observed within the splitter-plate boundary layer at the trailing edge. The latter peak matches the frequency at which spanwise vortices are shed from the planar shear-layer downstream of the trailing edge, and thus is attributed to the upstream influence of these vortices, transmitted via the pressure field. The lower-frequency peak at $S t_{\theta s}=0.0064$ agrees favourably with the frequency of the most-amplified perturbation predicted by the correlation of Walker 
(1994):

$$
f_{M A}=\frac{3.2 U_{1}^{2}}{2 \pi \nu R e_{\delta s^{*}}^{3 / 2}}
$$

where $f_{M A}$ is the frequency of the most-amplified perturbation, $R e_{\delta s^{*}}$ is the flow Reynolds number based on $U_{1}$ and the displacement thickness of the boundary layer in the high-speed stream at the splitter-plate trailing edge, and $\nu$ is the kinematic viscosity. Equation 4.1 predicts a value of $S t_{\theta_{s}, M A}=f_{M A} U_{1} / \theta_{s}=0.0068$. The $S t_{\theta s}=0.0064$ peak in the perturbation-velocity spectra is observed in each of the high Reynolds number cases, and is not observed in the low Reynolds number cases that are below the value of the critical flow Reynolds number.

\subsection{Instability Development in the Planar Free Shear Layer}

\subsubsection{Effect of Wall Proximity on the Instantaneous Flow Field}

To demonstrate the typical interaction observed between the free shear layer and the lower wall, Figure 4.4 presents the instantaneous spanwise vorticity distribution corresponding to the case with $R e_{\theta s}=320, R=1.88$ and $y_{s} / \theta_{s}=14.5$ in successive stages of one period $(\mathscr{T})$ of vortex-shedding. The figure shows the development of streamwise waviness of the shear layer caused by streamwise grouping of spanwise vorticity as driven by the Kelvin-Helmholtz $(\mathrm{K}-\mathrm{H})$ instability discussed in Chapter 2 . The vectors in Figure 4.4 show that during the development of the K-H instability, the wall-normal component of velocity increases until it reaches a threshold value, beyond which the shear-layer rolls-up into a discrete vortex, hereafter referred to as a $\mathrm{K}-\mathrm{H}$ vortex, that is shed downstream. The development of the wall-normal velocity 


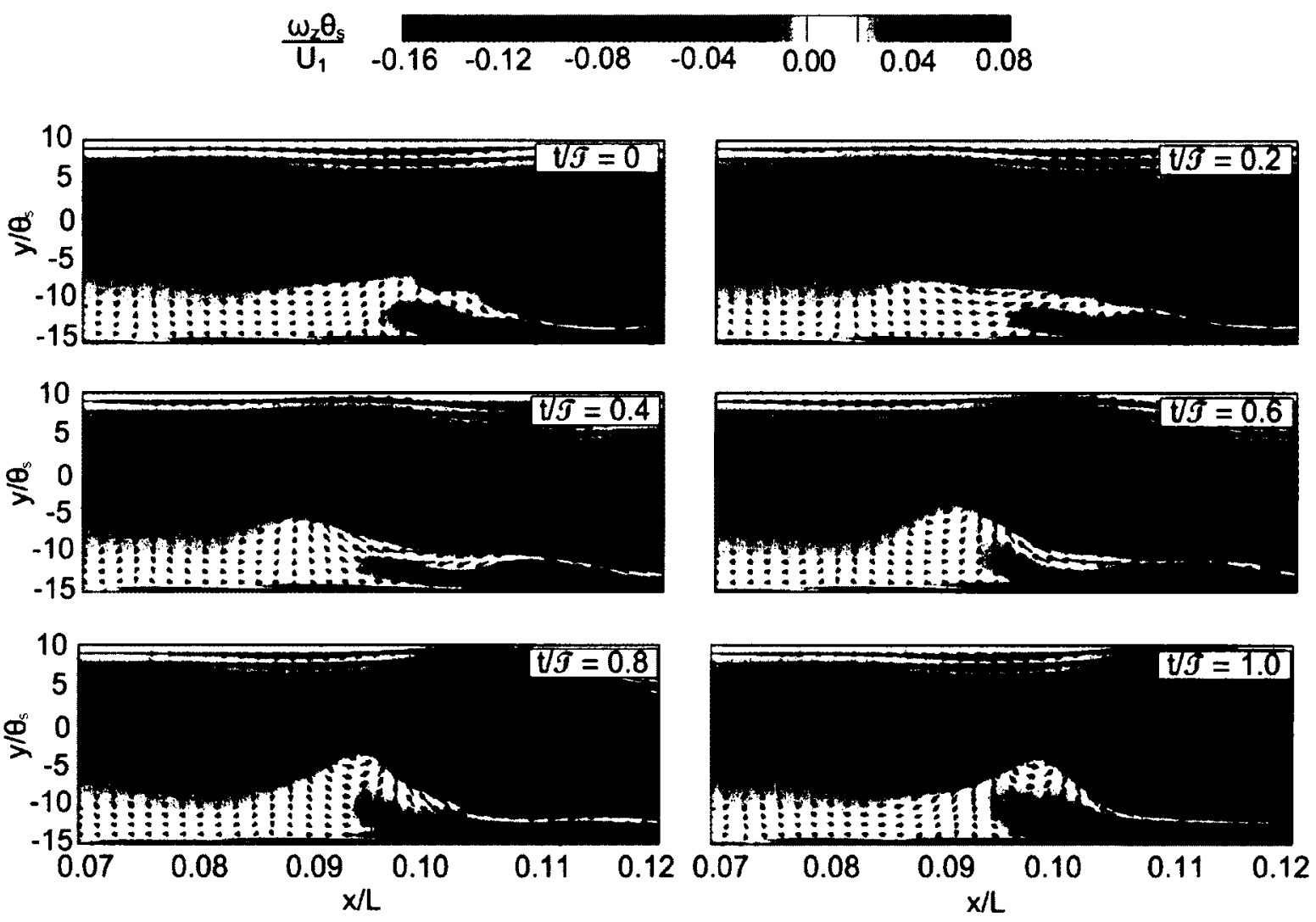

Figure 4.4: Instantaneous distribution of spanwise vorticity component for one cycle of vortex-shedding for $\operatorname{Re}_{\theta \mathrm{s}}=320, R=1.88, y_{\mathrm{s}} / \theta_{\mathrm{s}}=14.5$.

component $(v)$ is essential for the formation of $\mathrm{K}-\mathrm{H}$ vortices in the free shear layer, and an analysis of this component of velocity provides insight into the interaction mechanism between the shear layer and the lower wall. It is observed that the creation of a discrete vortex is accompanied by an increased level of induced reversed flow along the lower wall, creating positive signed vorticity along the lower wall that is of the opposite sign as the splitter-plate free-shear layer vorticity. These patches of induced vorticity at the wall are periodically ejected from the wall into the regions between the shed vortices, referred to as the "braid" region, and convect downstream with the velocity of the discrete vortices.

While the initial development of the planar free shear layer due to $\mathrm{K}-\mathrm{H}$ instability 


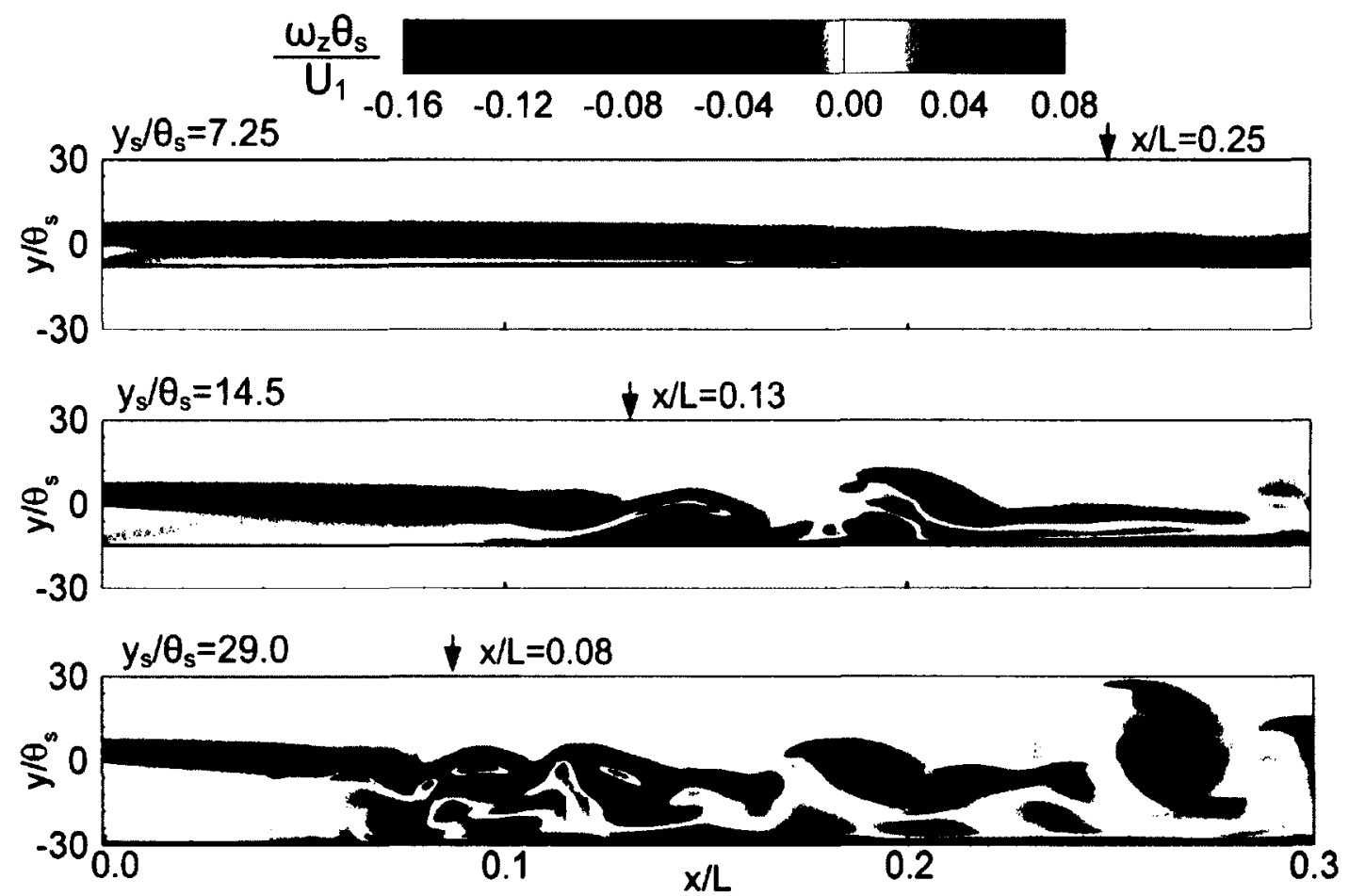

Figure 4.5: Instantaneous distribution of spanwise vorticity component for $\mathrm{Re}_{\theta_{\mathrm{s}}}=\mathbf{3 2 0}$ and $\mathrm{R}=\mathbf{1 . 8 8}$.

is expected to remain two-dimensional, three-dimensional flow features will eventually develop partly as a result of an interaction between the $\mathrm{K}-\mathrm{H}$ vortices and streamwise vortices that originate in the splitter-plate boundary layer (Brinkerhoff and Yaras, 2011). Following this interaction, breakdown to three-dimensional small-scale turbulence occurs in localized regions of high rates of shear (McAuliffe and Yaras, 2007). This three-dimensional breakdown cannot be reproduced by the present simulations in 2D space. Therefore, based on these previous studies, the streamwise extent of the present two-dimensional simulations that can reliably be used for studying the shear layer development are identified by arrows in Figures 4.5 to 4.7 .

The cases with $R e_{\theta s}=320$ and $R=1.88$ shown in Figure 4.5 have the greatest levels of instability manifested by the earliest onset of shear-layer roll-up, the most 

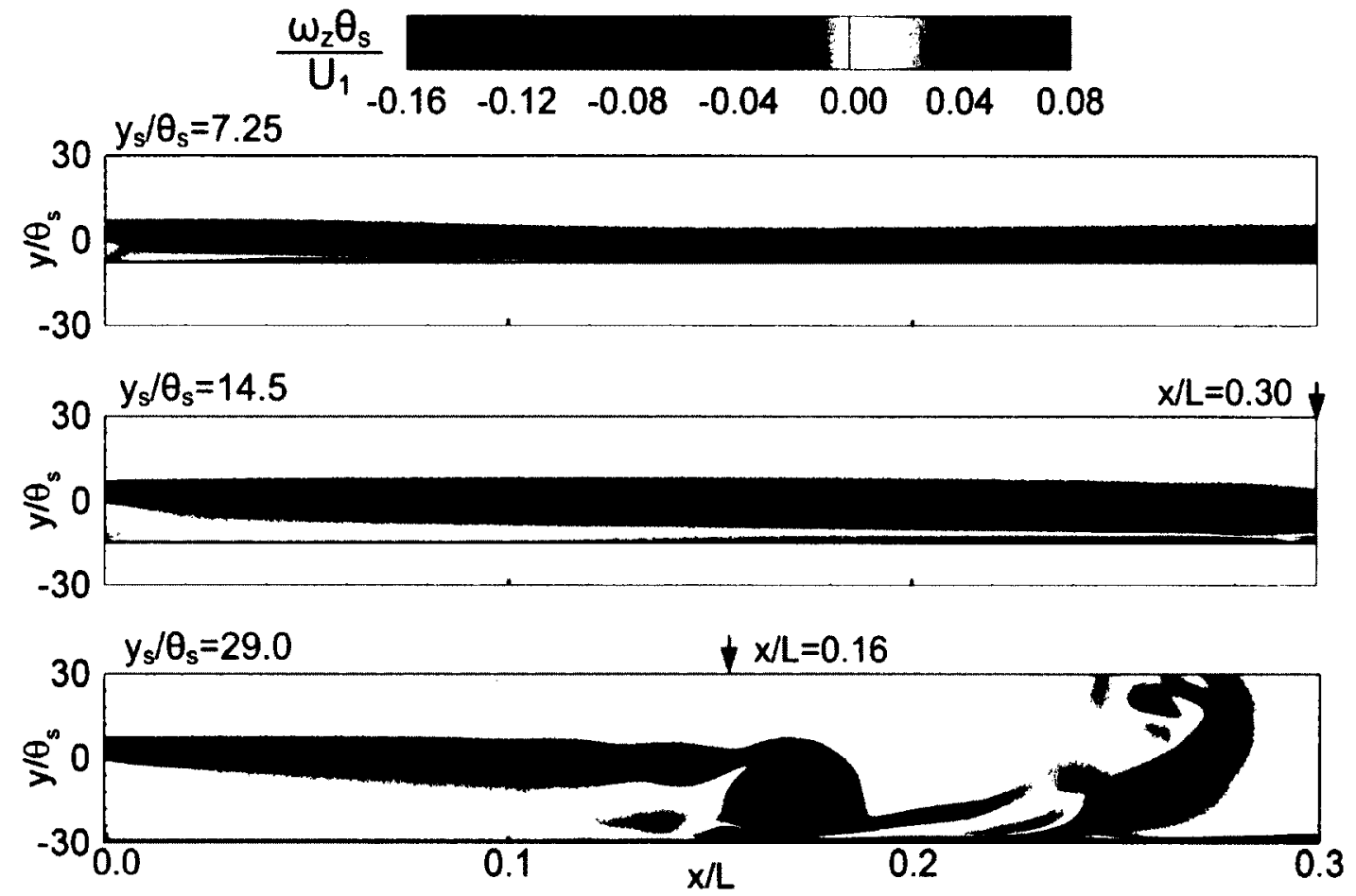

Figure 4.6: Instantaneous distribution of spanwise vorticity component for $\operatorname{Re}_{\theta s}=108$ and $\mathbf{R}=1.88$.

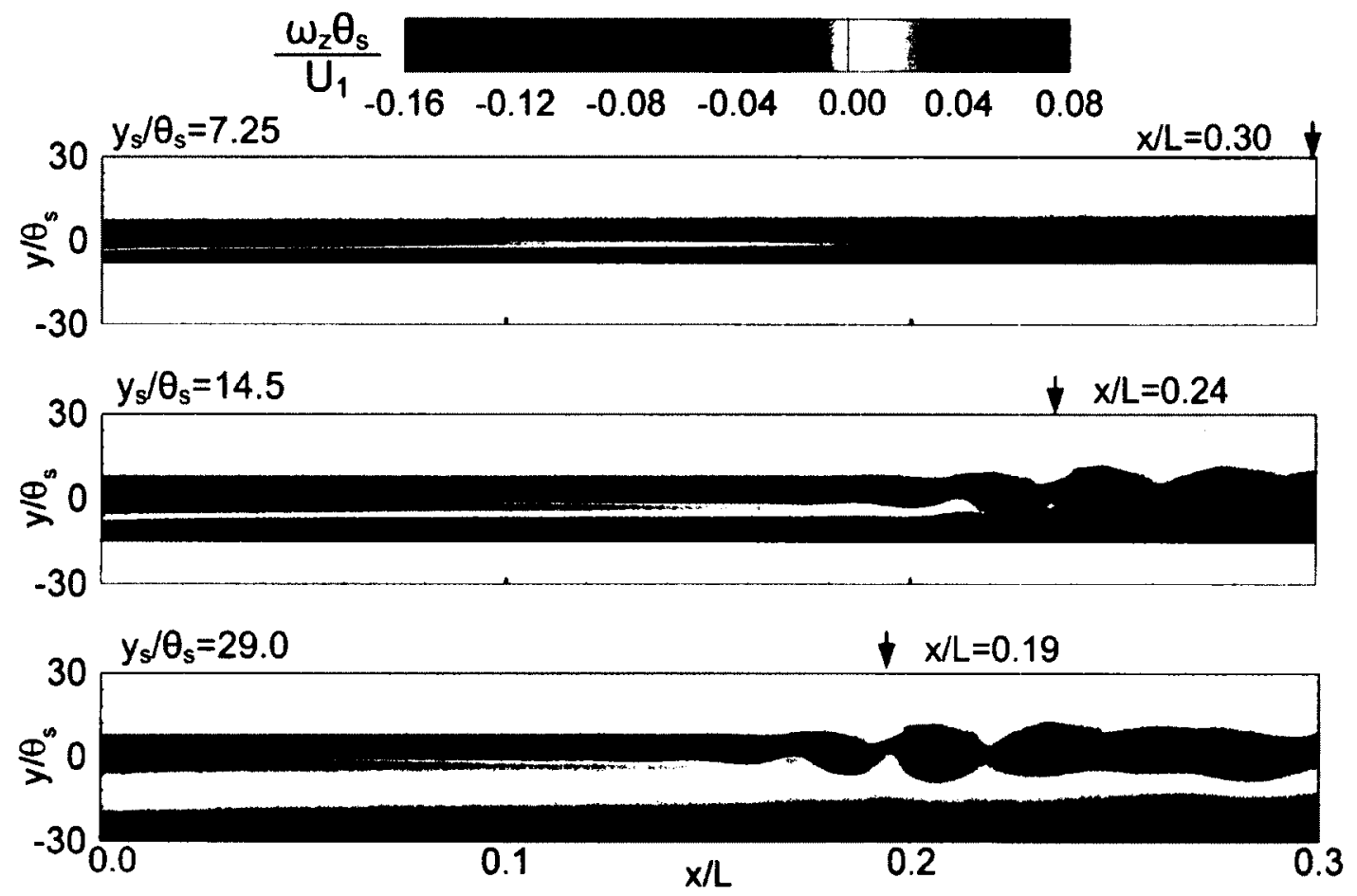

Figure 4.7: Instantaneous distribution of spanwise vorticity component for $\boldsymbol{R e}_{\theta \mathbf{s}}=320$ and $\mathbf{R}=\mathbf{1 . 2 2}$. 
complex vortex-wall interaction, and the most rapid breakdown compared to the other cases with lower $R e_{\theta s}$ and/or $R$ values presented in Figures 4.6 and 4.7. This result is consistent with the expectation of increased instability at higher flow Reynolds numbers and higher velocity difference ratios. Comparison of the cases in Figure 4.6 $\left(R e_{\theta s}=108\right.$ and $\left.R=1.88\right)$ with those in Figure $4.5\left(R e_{\theta s}=320\right.$ and $\left.R=1.88\right)$ shows increased sensitivity to wall proximity with reduced flow Reynolds number. This can be observed by the significant delay in the streamwise location of vortex roll-up between $y_{s} / \theta_{s}=14.5$ and $y_{s} / \theta_{s}=29.0$ in cases with $R e_{\theta_{s}}=108$ and $R=1.88$ compared to cases with $R e_{\theta s}=320$ and $R=1.88$. In the closest proximity case in Figure 4.6, no deformation of the shear-layer is observed in the vorticity contours. The cases with lower velocity ratio $\left(R e_{\theta s}=320\right.$ and $\left.R=1.22\right)$ shown in Figure 4.7 display a delayed streamwise location of shear-layer deformation compared to the cases with $R e_{\theta s}=320$ and $R=1.88$. This trend can be explained by recognizing that the lower velocity difference between the high-momentum and low-momentum streams leads to weaker $\mathrm{K}-\mathrm{H}$ instability due to the lower magnitude of velocity gradient at the inflection point, as is observed in several studies. For example, Michalke (1964) used inviscid linear stability analysis of a free shear layer to demonstrate that reduced velocity gradients due to a lower $R$ value result in a lower amplification rate of perturbations in the free shear layer.

For each combination of $R e_{\theta s}$ and $R$, the complexity of the interaction of the shed vortices with the wall and the rate at which the shear-layer reaches the state of break-down into turbulence increases with increased distance from the wall (i.e. higher $y_{s} / \theta_{s}$ values). In Figure 4.5, proximity to the wall is observed to delay the initial roll-up location in agreement with several published experimental and numerical studies of shear layers (Taghavi and Wazzan, 1974; Nayfeh et al., 1990; Das, 1998). As the shear layer is moved away from the wall, there is an increased spatial distribution of positive-sense vorticity convecting into the shear layer. Moreover, the 
closest proximity cases show significantly delayed roll-up, and roll-up is preceded by large segments of streamwise waviness that are connected by thick braids of vorticity. These trends may be the result of the vortices that form through roll-up of the free shear layer experiencing variations in their structure as distance to the wall is varied, and will be further analyzed in Sections 4.4 and 4.5 .

Figure 4.8 shows the streamwise distribution of the stream-normal perturbation velocity component. The streamwise growth rate of these perturbations is observed to be initially exponential in all cases. This rate of growth continues until the normalized perturbation velocity component $\left(v / U_{1}\right)$ reaches a value on the order of $10^{-3}$ to $10^{-2}$, which approximately coincides with streamwise location where the deformed shear layer completes the roll-up process forming discrete spanwise vortices. Despite the scatter in the data, the trend lines indicate an increase in the initial amplification

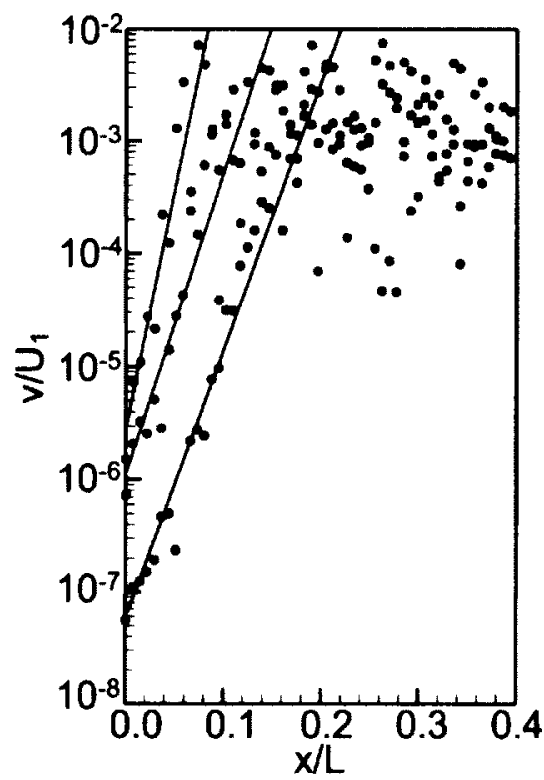

(a)

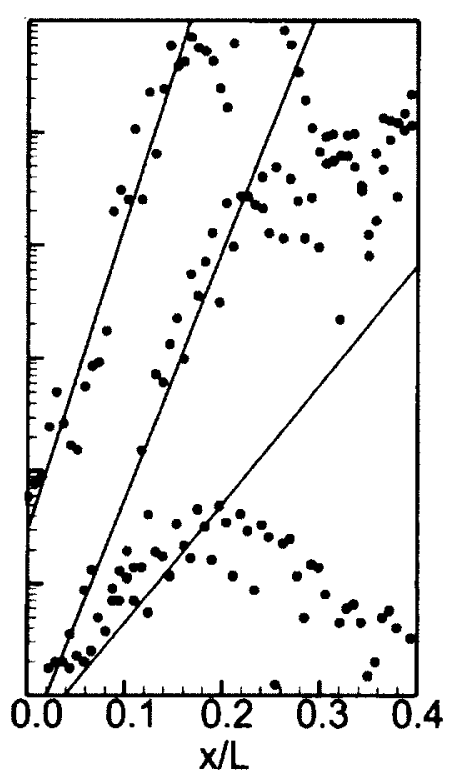

(b)

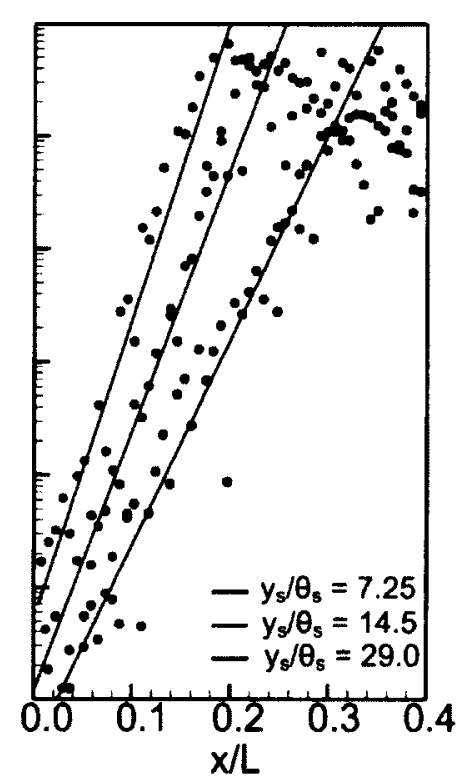

(c)

Figure 4.8: RMS of the wall-normal component of perturbation velocity (v) at $y / \theta_{s}=0$ for (a) $R_{\theta s}=320$ and $R=1.88$, (b) $R_{\theta s}=108$ and $R=1.88$, and (c) $\operatorname{Re}_{\theta \mathrm{s}}=320$ and $\mathrm{R}=1.22$. 
rate of the perturbations as the shear layer is moved away from the wall. This effect is most evident for the lower Reynolds number case, suggesting that the sensitivity of the shear layer development to wall proximity increases with reduced flow Reynolds number. Comparing Figure $4.8 \mathrm{a}$ and $4.8 \mathrm{c}$ shows that, for a given wall-proximity and Reynolds number, the amplification rate is higher in cases with a larger velocity ratio $\mathrm{R}$, which is in agreement with the observations of Michalke (1964).

\subsubsection{Effect of Wall Proximity on the Time-Averaged Flow Field}

Figures 4.9 to 4.11 show flood plots of the time-averaged streamwise velocity. The location of shear-layer attachment to the wall, defined as the most-upstream location on the wall where the time-averaged wall shear stress is positive (indicated in 4.9 to 4.11 by arrows where applicable), is observed to be a function of wall-proximity. Table 4.1 gives the streamwise location of flow attachment for cases with $R=1.88$, denoted $x_{r}$. The trends suggest that increased distance from the wall leads to more rapid deformation of the inviscid-unstable free shear layer and consequently an earlier site of flow attachment onto the wall. Low velocity-difference-ratio cases $(R=1.22)$ consistently have positive wall shear stresses, as the primary $\mathrm{K}-\mathrm{H}$ vortices never produce reverse flow at the wall for these cases and, consequently, a point of attachment can not be defined. The location of flow-attachment is consistently within one wavelength

Table 4.1: Streamwise location of flow attachment onto the wall for $R=1.88$.

\begin{tabular}{c|ccc}
\hline & $R e_{\theta s}=108$ & $R e_{\theta s}=320$ \\
$y_{s} / \theta_{s}$ & & $x_{r} / L$ & \\
\hline 7.25 & N/A & 0.23 \\
14.5 & 0.33 & 0.15 \\
29.0 & 0.20 & 0.10 \\
\hline
\end{tabular}




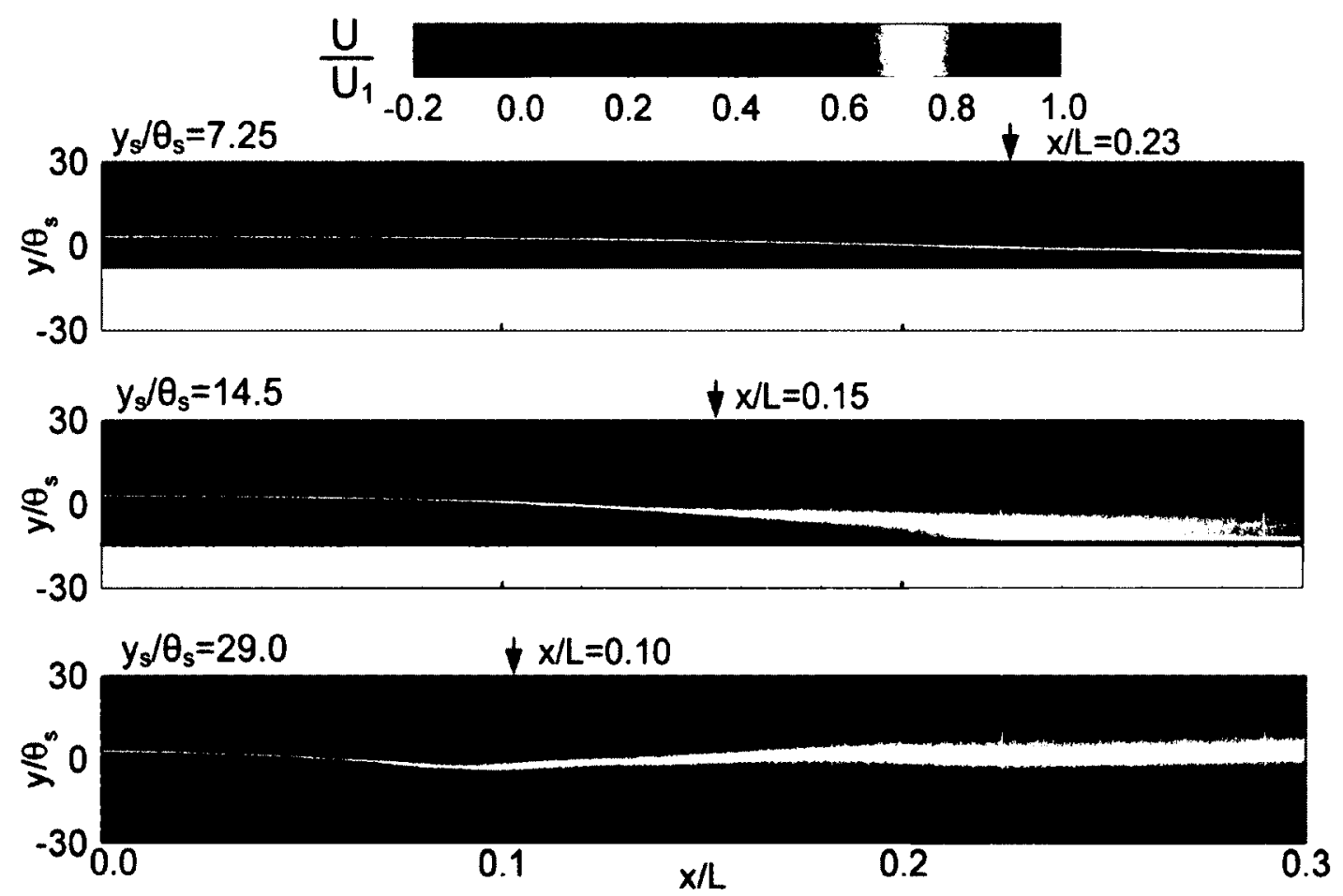

Figure 4.9: Time-averaged streamwise velocity for $R_{\theta \mathrm{s}}=320$ and $R=1.88$.

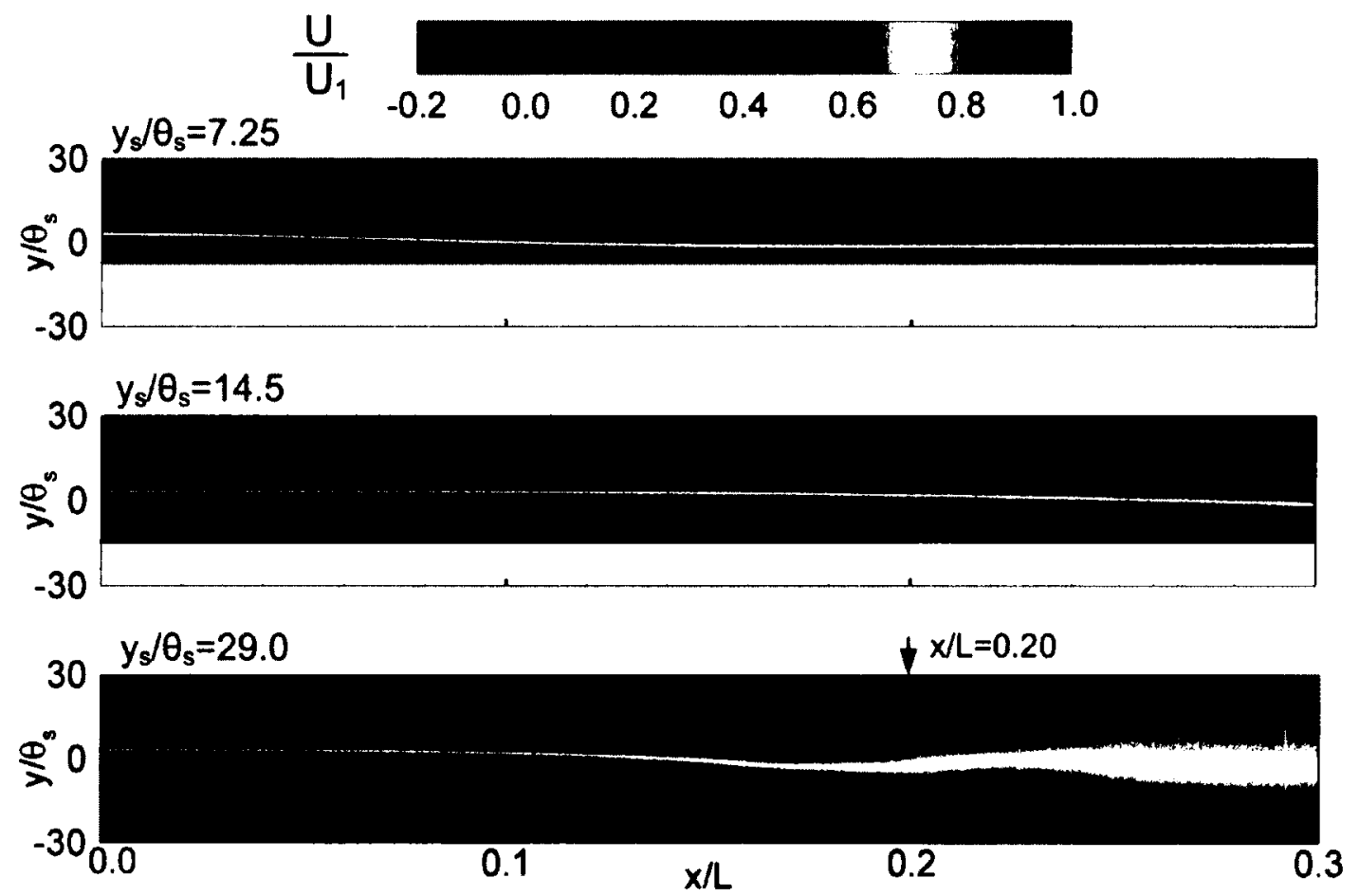

Figure 4.10: Time-averaged streamwise velocity for $\operatorname{Re}_{\theta_{\mathrm{s}}}=108$ and $\mathrm{R}=1.88$. 


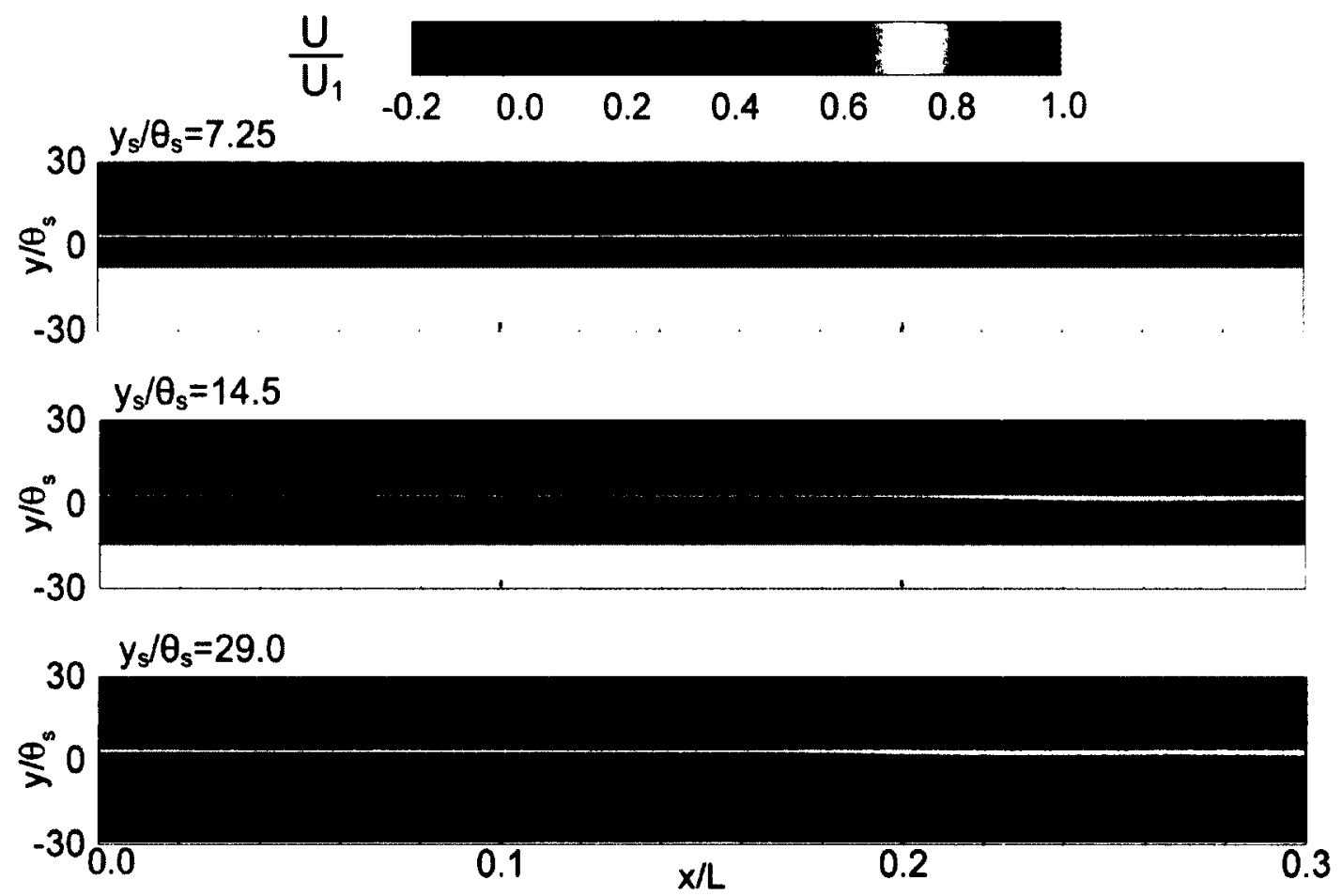

Figure 4.11: Time-averaged streamwise velocity for $\operatorname{Re}_{\theta \mathrm{s}}=320$ and $R=1.22$.

downstream of the location where the first $\mathrm{K}-\mathrm{H}$ vortex forms, suggesting that these vortices have a dominant role in the mixing and subsequent attachment of the shear layer to the wall. It is further noted that attachment onto the wall occurs only a very short distance downstream of the locations where three-dimensional instabilities are expected to form within the shear layer, as shown by the arrows in Figures $4.9-4.11$. Considering that a finite distance is required following the onset of three-dimensional instabilities for the resulting flow structures to have sufficient amplitudes to affect the shear-layer mixing, it is unlikely that attachment to the wall is significantly affected by these flow structures. Instead, the results suggest that the flow attachment process, in a time-averaged sense, is driven by the large-scale rollers produced by $\mathrm{K}-\mathrm{H}$ instability rather than the mixing associated with the three-dimensional breakdown 
of the shear layer.

\subsubsection{Effect of Wall Proximity on Transition Onset}

The flow configuration for the present study was chosen to complement published studies of laminar separation bubbles on airfoils (Mayle, 1991; Hatman and Wang, 1999; Roberts and Yaras, 2005; McAuliffe and Yaras, 2007). The trailing-edge flow field for the cases with $R=1.88$ correspond most closely to the time-averaged flow field just downstream of separation in typical laminar separation bubbles on airfoils. For example, McAuliffe and Yaras (2007), Rist and Augustin (2006) and Watmuff (1999) studied separation bubbles with $R \approx 1.8$. It is therefore of interest to compare the transition onset locations suggested by the present results to those predicted by semi-empirical correlations calibrated against airfoil separation-bubble data. Specifically, the transition onset locations given in the form of a local Reynolds number, $R e_{s-t r}$, for the present flows with $R=1.88$ are compared in Figure 4.12 against existing transition models for separation bubbles proposed by Mayle (1991), Hatman and Wang (1999), Roberts and Yaras (2005), and Praisner and Clark (2007). The comparison is based on the assumption that transition onset in the present simulated flows is driven by the development of spanwise-oriented vortices resulting from the $\mathrm{K}-\mathrm{H}$ instability, wherein breakdown to small-scale turbulence occurs within vortex braids, as discussed by McAuliffe and Yaras (2007). The results for $y_{s} / \theta_{s}=14.5$ and $y_{s} / \theta_{s}=29.0$ show the best agreement with the transition models. When considering accuracy and consistency of this accuracy of prediction, the models of Roberts and Yaras (2005) and Hatman and Wang (1999) appear to provide the most favourable predictions. Both models show good agreement at higher Reynolds numbers $\left(\operatorname{Re}_{\theta s}=320\right)$ for shear layers with $y_{s} / \theta_{s}=14.5$ and $y_{s} / \theta_{s}=29.0$ as demonstrated by a deviation from the simulation results of less than $15 \%$ in each case. At the low Reynolds number $\left(\operatorname{Re}_{\theta s}=108\right)$, the Roberts and Yaras model better 


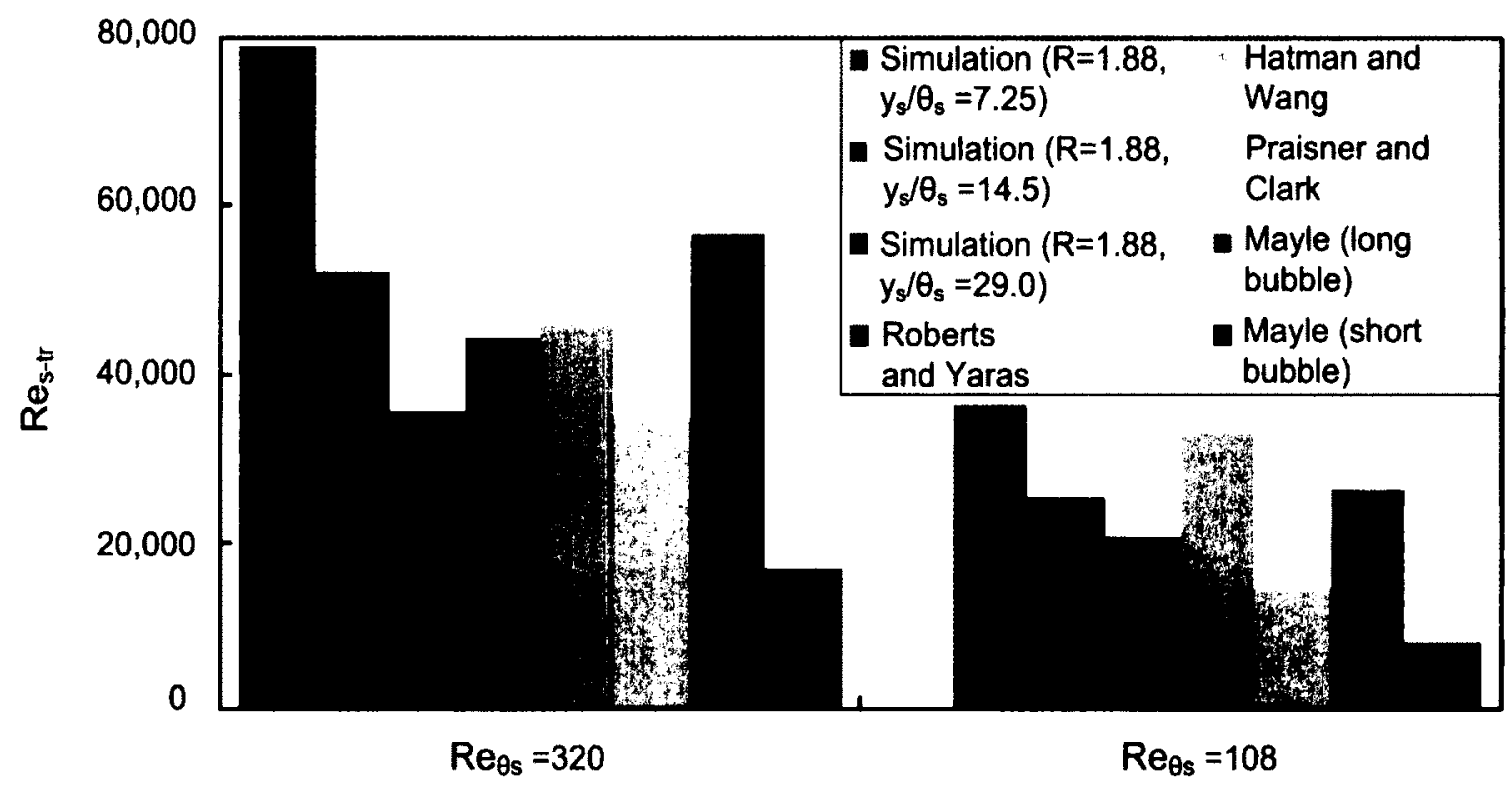

Figure 4.12: Computed $(R=1.88)$ and predicted values of transition onset Reynolds number.

predicts transition onset for $y_{s} / \theta_{s}=29.0$ while the Hatman and Wang model better predicts transition onset for $y_{s} / \theta_{s}=14.5$, with each model deviating from the corresponding simulation result by less than $15 \%$. Praisner and Clark's model generally under-predicts the location of transition onset, which is consistent with results noted by McAuliffe and Yaras (2007). Mayle's transition model for short separation bubbles greatly underestimates the location for transition onset. This tendency of the model has also been noted by Walker (1993). In contrast, the long-bubble model of Mayle is observed to provide better agreement with the simulation data corresponding to $y_{s} / \theta_{s}=7.25$. This result is not surprising given that long separation bubbles tend to be notably shallower than their short counterparts (Gostelow and Thomas, 2006). One instance of a long laminar separation bubble was studied by Gostelow and Thomas (2006), who observed that their long bubble was considerably thinner than short bubbles and therefore barely influenced the surrounding pressure field. The 
remaining transition models considered here do not fare well against the $y_{s} / \theta_{s}=7.25$ case. This is expected since these models were calibrated using experimental data for short separation bubbles which are more commonly observed on typical airfoils.

\subsubsection{Effect of Wall Proximity on the Frequency of Vortex Shedding}

The influence of wall-proximity on the stability of the planar shear-layer is further assessed by analyzing the frequency at which spanwise vortices are formed through the primary instability of the planar free shear-layer, obtained by monitoring the temporal variation of circulation inside a finite control volume within the flow-field. The finite control volume, shown in Figure 4.13, is located so that shed spanwise vortices pass through the volume as they are convected downstream. The volume dimensions are one wavelength of the primary $\mathrm{K}-\mathrm{H}$ mode in the streamwise direction and $20 \theta_{s}$ in the wall-normal direction. These dimensions are conservatively chosen for one discrete spanwise vortex to be fully enclosed within the volume. As a discrete vortex enters the volume, the circulation within the volume increases, reaching a maximum when the discrete vortex is completely inside the volume. Monitoring the circulation variation within the volume in time produces a transient signal with

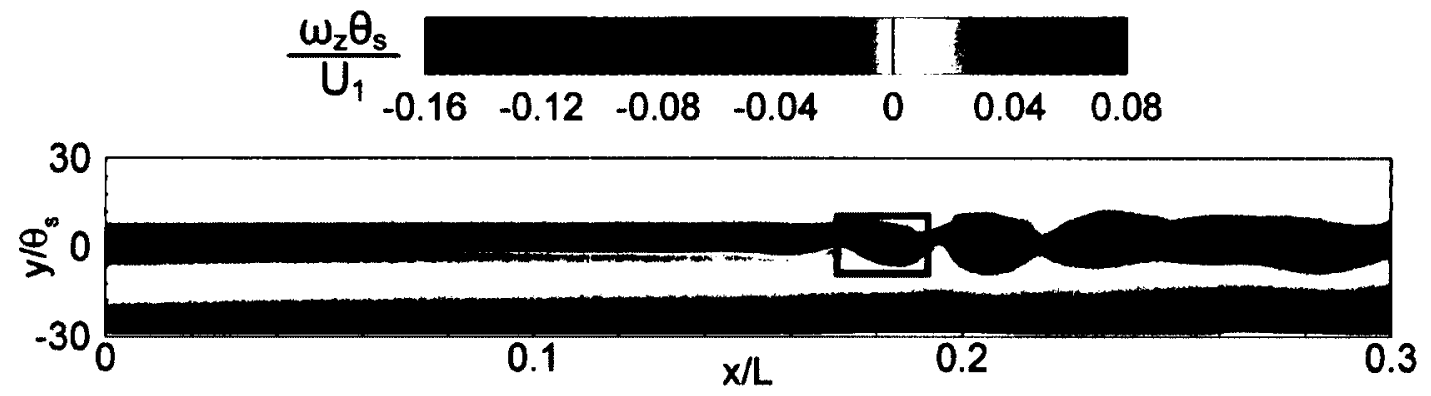

Figure 4.13: Sample illustration of the control volume used for monitoring the vortex-shedding frequency. 


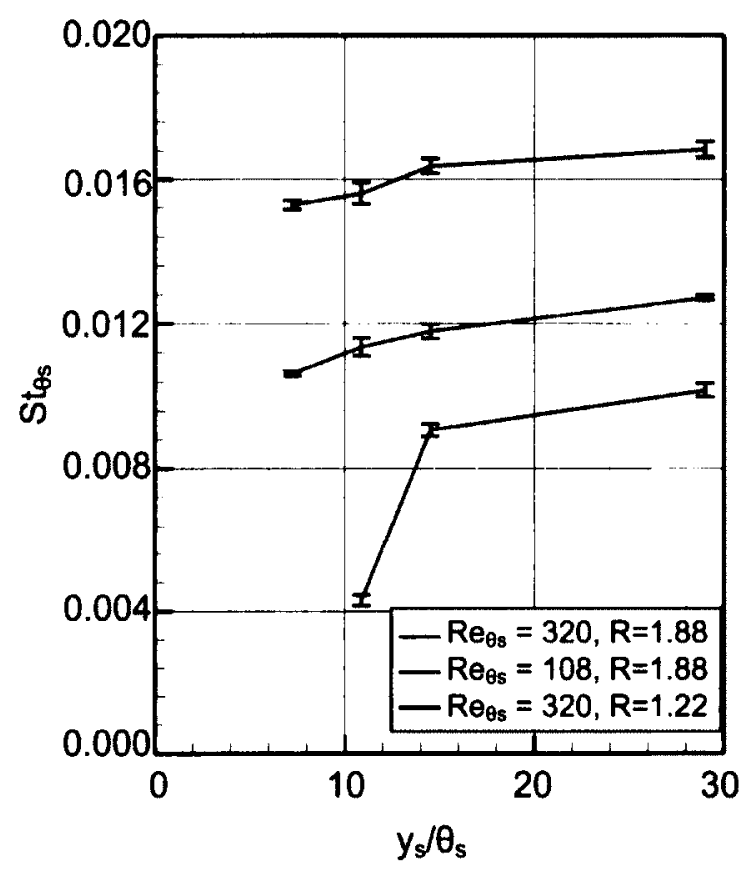

Figure 4.14: Variation of vortex shedding frequency with wall proximity.

periodic peaks corresponding to the passage of vortices. Analysis of this signal yields the mean and standard deviation of the frequency at which vortices are shed from the planar shear-layer. This method was adopted over spectral analysis of point measurements because the use of a finite flow region rather than a single point in the construction of the signal reduces the signal noise.

The computed shedding frequencies are shown in Figure 4.14. The shedding frequency is normalized by $\theta_{s}$ and $U_{1}$ to form the Strouhal number. The uncertainty of the vortex shedding frequency, shown by an error bar in Figure 4.14, was quantified by analysing the spread in vortex shedding frequencies over 100 shedding cycles, as detailed in Appendix B. The range of computed Strouhal numbers agree well with the ranges observed in published studies of separation bubbles and free-shear-layers, listed in Table 4.2. The low Strouhal number range observed by Muti Lin and Pauley (2006) occurs because of the low value of the momentum-thickness Reynolds number, 
comparable to the low Reynolds number cases in the current study in which a very similar Strouhal number range is obtained. In cases with a low velocity-difference ratio, the mean velocity in the mixing region downstream of the splitter-plate is larger than in the high velocity-difference cases. As a result, the discrete vortices shed from the shear-layer in the low velocity-difference-ratio cases are convected through the finite sampling control volume at a higher frequency. As the Strouhal number in Figure 4.14 is normalized by $U_{1}$ rather than the local mean convection velocity, this produces the larger Strouhal number values observed in Figure 4.14 for the low velocity-difference-ratio cases. The trends in Figure 4.14 clearly show a correlation between the mean shedding frequency and the proximity of the shear layer to the wall. This sensitivity is noted to increase with closer proximity of the shear layer to the wall. This trend is consistent with the findings of Diwan and Ramesh (2009) who, through linear stability analysis of a piecewise-discontinuous profile, observed that the shedding frequency becomes insensitive to wall proximity above a threshold

Table 4.2: Published Strouhal number ranges in studies of separation bubbles and free-shear-layers.

\begin{tabular}{lll}
\hline Study & Geometry & St $_{\theta_{\text {s }}}$ \\
\hline \hline McAulliffe and Yaras (2007) & separation bubble & $0.008-0.016$ \\
Muti Lin and Pauley (1996) & separation bubble & $0.005-0.008$ \\
Yang and Voke (2001) & separation bubble & $0.005-0.011$ \\
Talan and Hourmouziadis (2002) & separation bubble & $0.010-0.014$ \\
Ho and Heurre (1984) & free-shear-layer & 0.016 \\
\hline
\end{tabular}

proximity of approximately $20 \theta_{s}$. 


\subsection{Linear Stability Analysis}

Dovgal et al. (1994), Rist and Maucher (2002) and McAuliffe and Yaras (2007) suggest that the stability of shear layers in close proximity to walls is modified by T-S instability at the wall. Figure 4.15 plots the time-averaged and perturbation velocity profiles, one with $\left(y_{s} / \theta_{s}=7.25\right)$ close proximity to the wall and the second with a far proximity $\left(y_{s} / \theta_{s}=29\right)$, at a streamwise location that is one primary K-H instability wavelength upstream of the location where the separated shear-layer rolls-up into discrete vortices. The perturbation mode shapes show that the perturbation amplitude reaches a maximum near the wall for the closest wall-proximity case and near the planar shear-layer for the farthest wall-proximity. This suggests that perturbations in the close wall-proximity case grow primarily in the reversed-flow boundary layer that develops on the wall below the inflection point of the shear-layer and are amplified primarily by a viscous T-S instability mode. Conversely, perturbations in the farthest wall-proximity case grow primarily in the separated shear-layer and are mostly amplified by an inviscid K-H instability mode in the shear-layer. The wall-proximity thus influences the relative dominance of the viscous and inviscid instability modes in amplifying perturbations in the flow-field. A similar trend was observed by Rist and Maucher (2002) in a linear stability analysis of a hyperbolic-tangent velocity profile in close proximity to a wall. They observed that in instances with the inflection point of the mean velocity profile far from the wall, the perturbation velocity profile resembles that of a free-shear-layer. However, when the inflection point is moved near the wall, the instability changes to a viscous mode and the perturbation velocity profile resembles a T-S wave. As the viscous mode in their study is unstable to a lower frequency perturbation than the inviscid mode, the dominance of the viscous mode near the wall produces lower vortex-shedding frequencies.

To establish the extent by which viscous influence on the velocity profile affects 

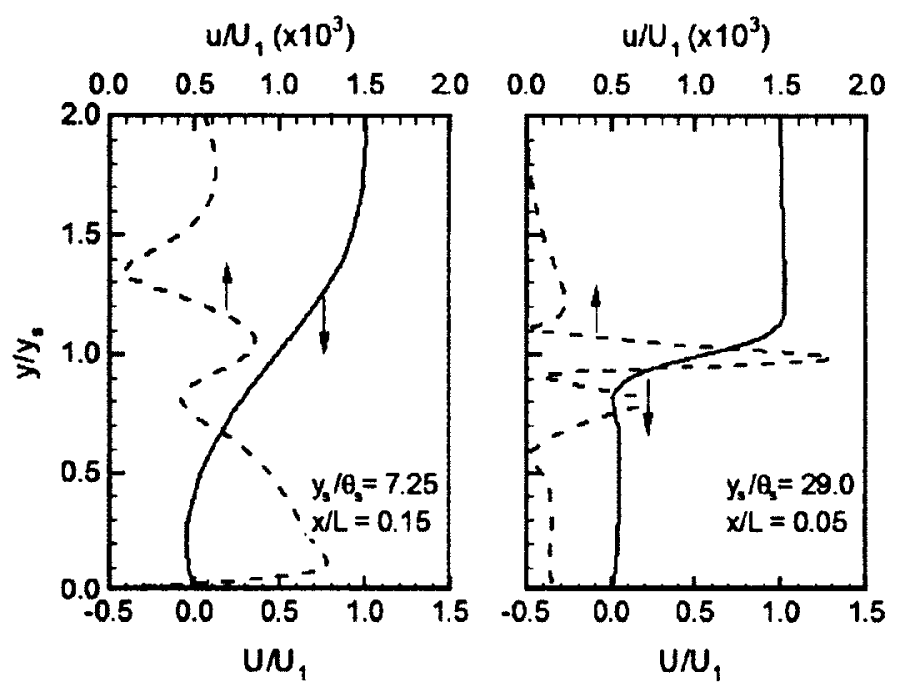

Figure 4.15: Time-mean and perturbation velocity profiles for $\operatorname{Re}_{\theta \mathrm{s}}=\mathbf{3 2 0}$, $R=\mathbf{1 . 8 8}$ at two wall proximities.

the frequency of the most amplified inviscid instability, a linear stability analysis is performed on the time-averaged velocity profile one wavelength upstream of rollup. This position is advantageous because one wavelength upstream of roll-up, the wall-normal component of velocity is small enough that the assumption of plane parallel flow is reasonably satisfied. The equations governing the temporal growth of perturbations are solved numerically following linear stability theory, which assumes a perturbation in the form of a two-dimensional wave with a real wavenumber and complex phase velocity. For a perturbation to grow in time, the imaginary component of the complex phase velocity must be positive. A hybrid spectral collocation method based on Chebychev polynomials, described by Schmid and Henningson (2001), is used to calculate the eigenvalues of the Orr-Sommerfeld and Squire equations for the near-wall portion of the computed time-averaged velocity profiles as shown in Figure 4.15. The code for this analysis was developed by Joshua Brinkerhoff, PhD candidate in the author's research group at Carleton University. By solving for the phase velocity and amplification rate over a frequency range of 10 to $1000 \mathrm{~Hz}$, the perturbation 
Table 4.3: Comparison of Strouhal numbers of the most-amplified perturbation at the streamwise locations in Figure 4.15, obtained from linear stability theory (LST) and direct numerical simulation (DNS).

\begin{tabular}{cll}
\hline$y_{s} / \theta_{s}$ & $S t_{\theta_{s}, L S T}$ & $S t_{\theta_{s}, D N S}$ \\
\hline \hline 7.25 & 0.0066 & 0.0106 \\
29.0 & 0.0118 & 0.0127 \\
\hline
\end{tabular}

frequency yielding the highest amplification rate of T-S instability mode is predicted. Table 4.3 compares the frequencies of most amplified perturbation (expressed as a Strouhal number $\left.S t_{\theta_{s}, L S T}\right)$ predicted from linear stability theory with the vortexshedding frequencies obtained from the simulations. Although the linear stability analysis underpredicts the most amplified Strouhal number, it correctly captures the trend in the Strouhal number with wall proximity. Furthermore, the trends in wallnormal variation of the predicted perturbation velocity agrees favourably with the simulation results shown in Figure 4.15 in both the mode shape and the wall-normal location of the maximum perturbation velocity. As the shear-layer is brought closer to the wall, the T-S instability mode is noted to have an increasingly larger influence on the shedding frequency of the vortices.

The dominance of the viscous instability mode for close wall proximities suggest that the reduction in frequency is fundamentally a viscous phenomenon where the frequency shifts from a maximum corresponding to the free-shear-layer instability value to a minimum corresponding to the attached boundary layer instability. In effect, the wall-proximity acts to dampen the natural frequency of vortex shedding through the influence of viscosity. 


\subsection{Transient Potential Flow Analysis}

A transient potential flow model is used to assess the role of inviscid mechanisms in delaying free-shear-layer roll up in close proximity of a wall. A detailed explanation of the transient potential flow algorithm is provided in Section 3.3.2. Figure 4.16 shows the instability development in a vortex sheet perturbed by a disturbance of the form $y=y_{p} \sin \left(2 \pi x / \lambda_{x}\right)$ with $\lambda_{x}=8 \mathrm{~mm}$ for a duration of $0.4 \mathscr{T}$, where $\mathscr{T}$ is one period between sequential vortex roll-ups. These shear-layers have undefined momentum thicknesses, consequently, wall proximities are normalized by the initial wall-normal perturbation displacement amplitude $\left(y_{p}=0.4 \mathrm{~mm}\right)$.

The study considers three cases: (a) a free-shear-layer unaffected by a wall $\left(y_{s} / y_{p}=\infty\right)$; (b) a shear-layer in far proximity to a wall $\left(y_{s} / y_{p}=1000\right)$; (c) a shearlayer in close proximity to a wall $\left(y_{s} / y_{p}=250\right)$. Over the duration of the simulation, the vorticity sheet representing the free shear-layer rotates around the meanline of the shear layer with mildly reduced amplitudes owing to the dissipative nature of the computational scheme. Similarly, the vorticity sheet with $\left(y_{s} / y_{p}=1000\right)$ rotates one full cycle with mild distortions in the vortex sheet compared to (a). The distortions suggest that the wall slightly influences the development of vortex roll-up. However, for $y_{s} / y_{p}=250$ the presence of the wall inhibits the development of roll-up and the shear layer can not rotate around the meanline. A close examination of a point (P) on the shear layer in Figure 4.16 reveals that the mechanism of the suppressed roll-up is inhibition of the wall-normal motion. The lower segment of the free-shear-layer vortex pattern is able to complete a rotation because the induced wall-normal velocity at the tip of the vortex pattern is sufficient to traverse the midline. However, the wall-normal component in the lower segment of the $y_{s} / y_{p}=250$ case does not have sufficient induced velocity to traverse the midline of the vortex pattern. 


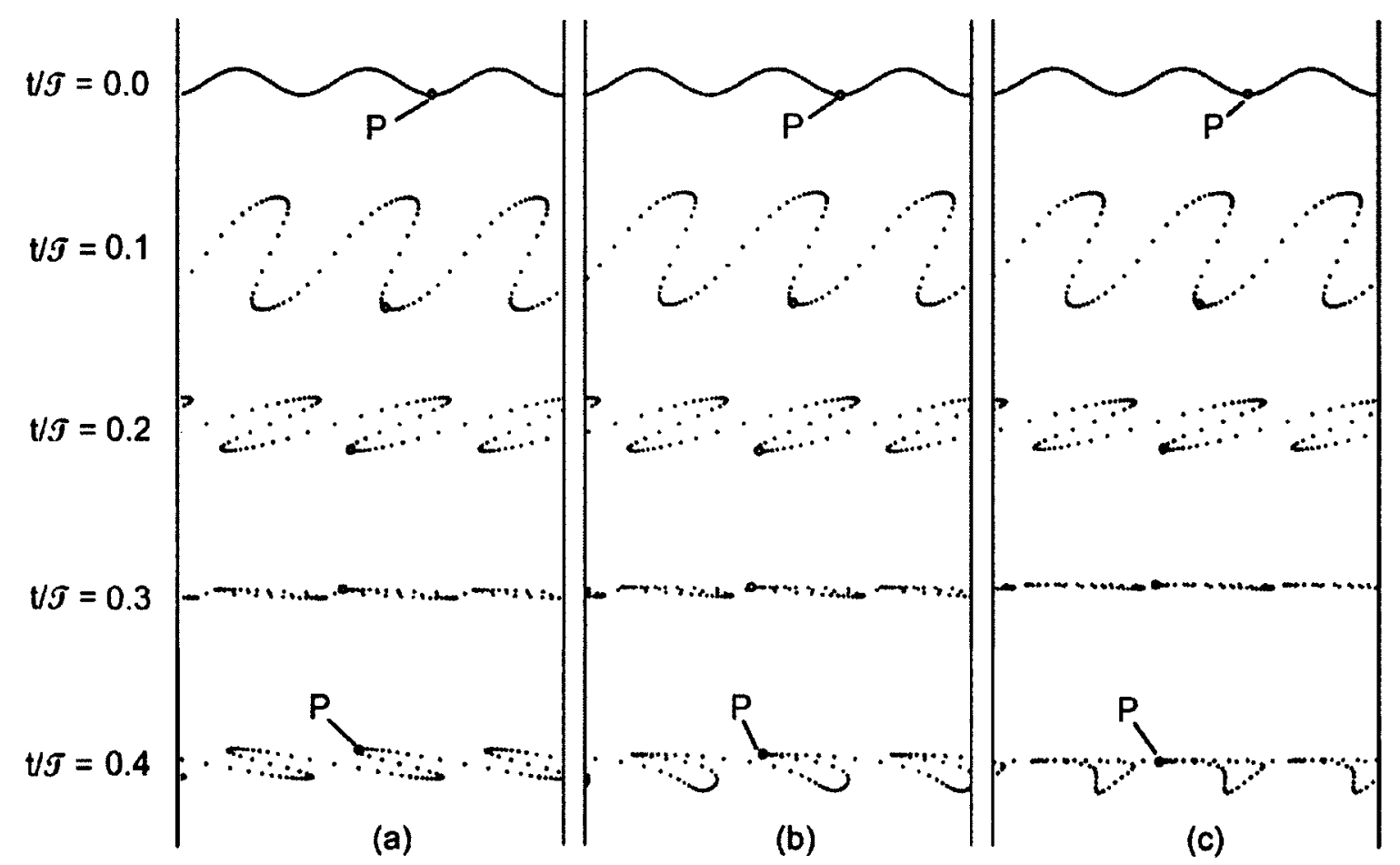

Figure 4.16: Shear layer development predicted through a transient potential-flow analysis for (a) free-shear-layer, (b) far wall-proximity $\left(y_{s} / y_{p}=1000\right)$, and (c) close wall-proximity $\left(y_{s} / y_{p}=250\right)$ where $\mathscr{T}$ is one period of vortex shedding.

\subsection{Steady Potential Flow Analysis}

Features of the shear-layer development in Section 4.2 may be explained with the aid of potential flow theory by modelling instantaneous snapshots of the unsteady velocity/vorticity field as quasy-steady states of flow. The stages of vortex roll-up consist of an initially flat vortex sheet, followed by the development of a streamwisewavy vortex-sheet pattern (again, not in shape but in vorticity magnitude) induced by unstable Kelvin-Helmholtz (K-H) waves, and finally roll-up into K-H vortices. Each stage in the vortex roll-up is modeled using potential flow elements, as discussed in Section 3.2.1, and the velocity profile below these vorticity distributions is shown in Figure 4.17. The figure shows that the reversed flow at the wall is higher in the stage where the vortex rolls up, modeled by a Rankine vortex and a point vortex in 


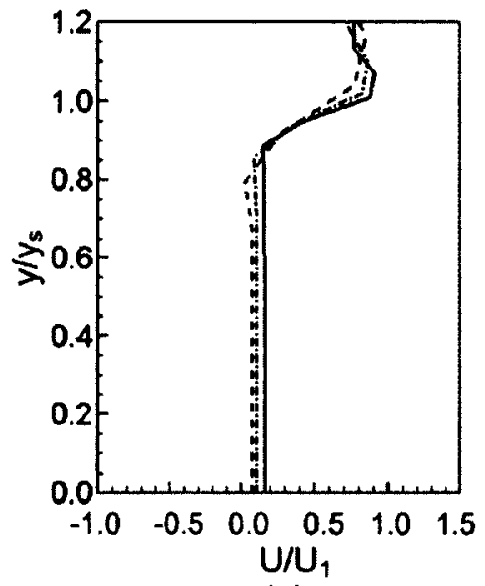

(a)

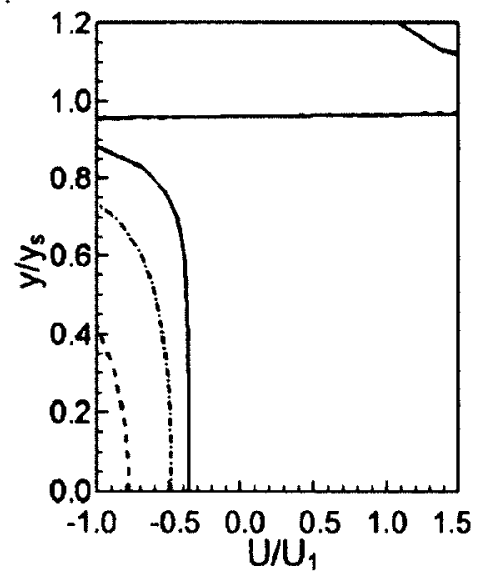

(c)

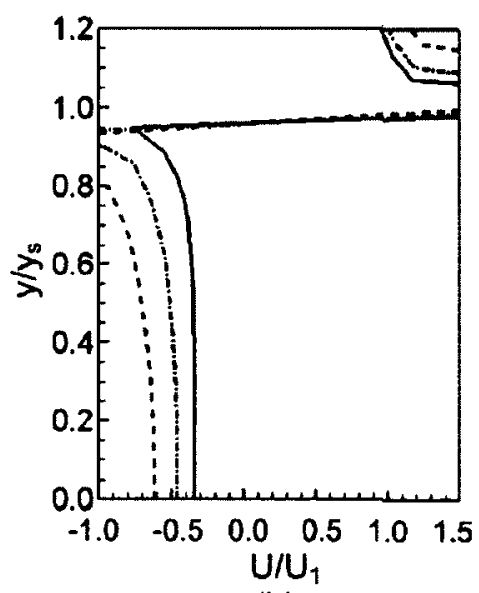

(b)

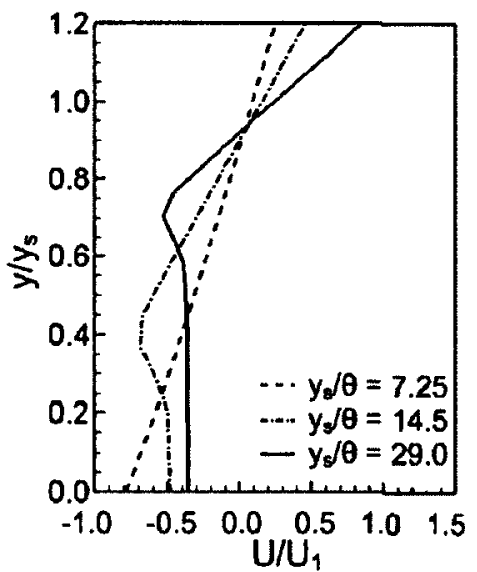

(d)

Figure 4.17: Wall-normal distribution of streamwise velocity under locations of maximum circulation for (a) flat vortex sheet, (b) streamwise-wavy vorticity pattern, (c) point vortex, and (d) Rankine vortex. 
Figure 4.17, despite keeping the total circulation in each vorticity distribution the same. This may be explained by the higher local concentration of circulation in a rolled-up vortex compared to a vortex sheet. Consequently, the earlier occurrence of positive sense vorticity in the larger wall-proximity cases in Figure 4.9 -4.11 is due to the earlier formation of vortices in these cases. Furthermore, the presence of higher wall-normal velocity in the larger wall-proximity cases facilitate more effective ejection of positive-sense vorticity away from the wall. A closer analysis of Figure 4.17 also shows that as the vortex patterns are brought closer to the wall, the induced reverse flow at the wall increases. The higher local reverse flow directly above the wall in the close proximity cases contributes to stronger Tollmien-Schlichting (T-S) instability at the wall. 


\section{Chapter 5}

\section{Effect of Supercritical Thermodynamic}

\section{State on Free Shear Layer Development}

This chapter discusses the stability of a planar shear layer in a supercritical fluid with a cross-stream temperature gradient using a numerical study in three-dimensional (3D) space. The effect of the supercritical state on the stability of the shear layer is isolated through comparison with a companion study of a planar shear layer of a subcritical single-phase fluid with constant fluid properties. The chapter begins with an overview of the subcritical and supercritical free-shear-layer simulations compared in this study. Section 5.2 discusses the primary and secondary instability modes observed in the subcritical free-shear-layer simulation, while Section 5.3 discusses the effect of the cross-stream variation in fluid properties within the supercritical fluid on these instability modes. 


\subsection{Summary of Flow Configurations}

Table 5.1 summarizes the pertinent flow characteristics of the simulated planar free shear layers utilizing working fluids at subcritical single-phase and supercritical thermodynamic states. For brevity, hereafter these planar shear layers will be referred to as the subcritical and supercritical shear layers. The subcritical shear-layer simulation uses air at $101.3 \mathrm{kPa}$ and $300 \mathrm{~K}$ as the working fluid, and the supercritical shear layer simulation uses supercritical water at $24 \mathrm{MPa}$. As in Chapter 4, the planar shear layers are created through the mixing of two parallel streams with different velocities separated by a splitter plate of infinitesimal thickness. Matching the Reynolds numbers of the two shear layers $\left(R e_{\theta s}=320\right)$, which is based on the velocity and momentum thickness of the high-speed stream at the trailing-edge of the splitter plate, and the velocity-difference ratio between the two parallel streams $(R=1.22)$

Table 5.1: Flow conditions of the subcritical and supercritical free shear layer simulations

\begin{tabular}{lcc}
\hline Parameter & Subcritical & Supercritical \\
\hline \hline$R e_{\theta s}$ & 320 & 320 \\
$R e_{\theta f}$ & 1000 & 950 \\
$R$ & 1.22 & 1.22 \\
$P r$ & 0.71 & 2.14 \\
$T_{m} / T_{p c}$ & N/A & 1 \\
$U_{1}(\mathrm{~m} / \mathrm{s})$ & 11.8 & 0.108 \\
$U_{2}(\mathrm{~m} / \mathrm{s})$ & 2.7 & 0.026 \\
$\theta_{s}(\mathrm{~mm})$ & 0.41 & 0.35 \\
$\theta_{f}(\mathrm{~mm})$ & 1.20 & 0.92 \\
$\delta_{\omega}(\mathrm{mm})$ & 7.0 & 4.5 \\
\hline
\end{tabular}




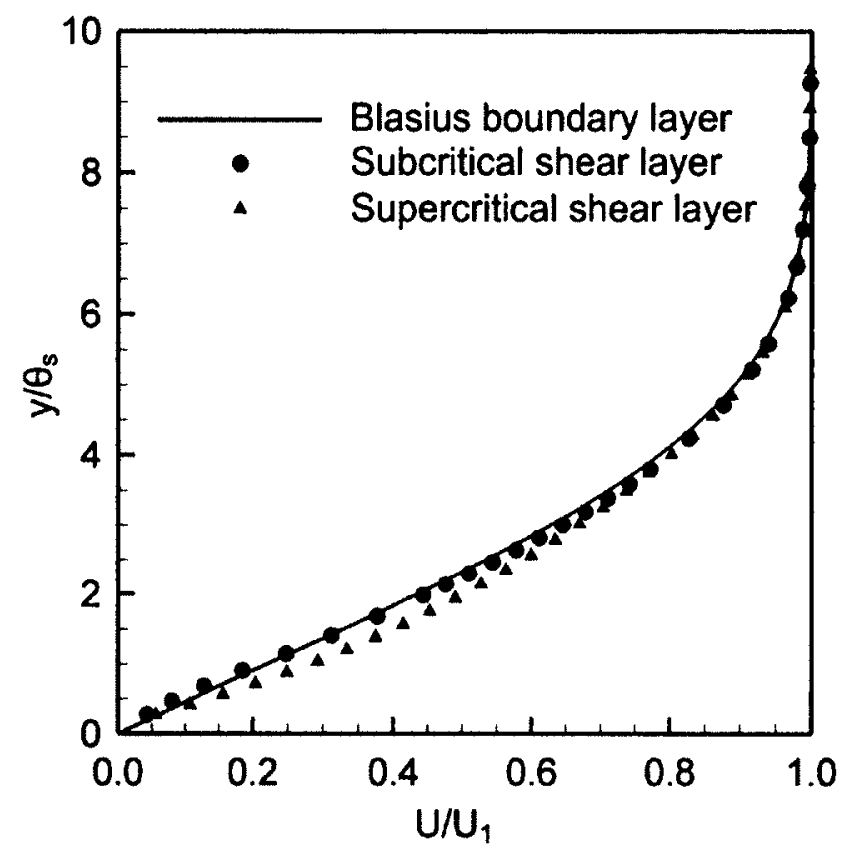

Figure 5.1: Splitter-plate high-speed-stream boundary layer velocity profile at the trailing edge

ensures that differences in the evolution of the flow field between the two cases can be attributed to the cross-stream property variation prevailing in the flow field of the supercritical shear layer. In the supercritical shear layer simulation, the thermodynamic properties used to calculate the Reynolds number $\left(R e_{\theta s}\right)$ and Prandtl number $(\operatorname{Pr})$ were determined at the algebraic mean of the high- and low-momentum streams. As shown in Figure 5.1, the high-speed-stream boundary layer at the splitter-plate trailing edge agrees well with the Blasius profile for the subcritical case, whereas the supercritical case shows slight deviation from the Blasius profile. Studies of free shear layers traditionally use the shear-layer momentum thickness $\left(\theta_{f}\right)$ as defined below as the appropriate length scale of the flow (e.g. Oster and Wygnanski, 1982; Ho and 

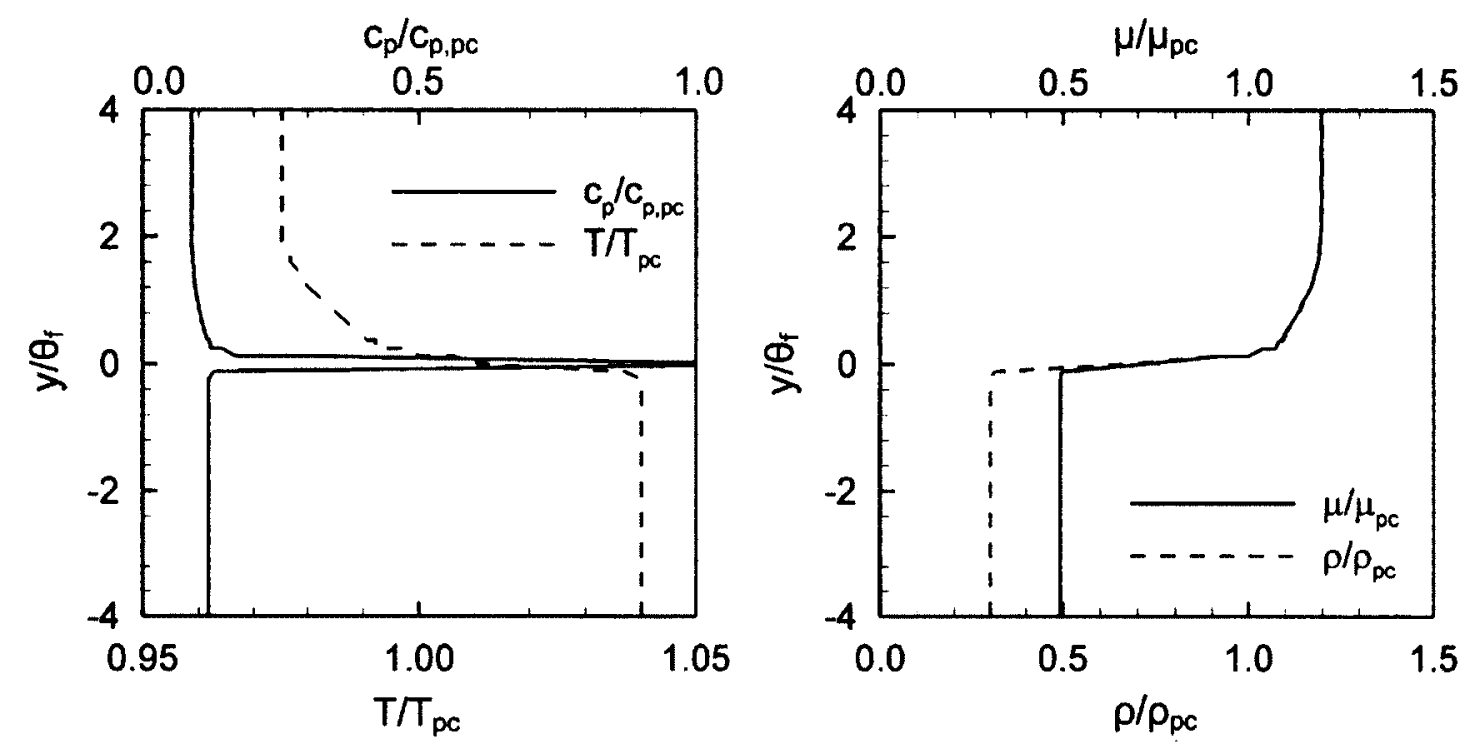

Figure 5.2: Cross-stream variation in temperature $(T)$, isobaric heat capacity $\left(\mathbf{c}_{\mathbf{p}}\right)$, density $(\rho)$ and viscosity $(\mu)$ in the supercritical shear layer.

Huang, 1983).

$$
\theta_{f}=\int_{-0.05 L}^{0.05 L} \frac{\rho(y) U(y)-\rho_{1} U_{1}}{\rho_{2} U_{2}-\rho_{1} U_{1}}\left(1-\frac{U(y)-U_{1}}{U_{2}-U_{1}}\right) d y
$$

Therefore, $\theta_{f}$ is used as the reference length scale in the results presented hereafter. Both the free-shear-layer momentum thickness, $\theta_{f}$, and vorticity thickness, $\delta_{\omega}=\left(U_{2}-U_{1}\right) /\left(d U /\left.d y\right|_{\max }\right)$, listed in Table 5.1 are calculated prior to the onset of roll-up where the shear layer velocity profile can be considered steady, which corresponds to approximately $x / L=0.05$ in the present simulations.

The variation across the supercritical shear layer, of density, viscosity, conductivity, and isobaric heat capacity at the trailing edge of the splitter plate are shown in Figure 5.2. The fluid temperature is noted to vary from below the pseudocritical temperature $T_{p c}$ in the low-momentum stream to above $T_{p c}$ in the high-momentum stream, assuming a value of $T_{p c}$ at the datum line $(y=0)$. Accordingly, the fluid 
properties experience their highest spatial gradients in close vicinity of the datum line which places these large-gradients within the free shear layer developing immediately downstream of the splitter-plate trailing edge. This configuration promotes development of the free-shear layer in the region of high thermophysical property gradients, which is expected to maximize any effects such gradients may have on the streamwise development of the shear layer.

\subsection{Instability Development in the Subcritical Shear Layer}

In the subcritical shear-layer simulation, the high- and low-momentum streams meet at the splitter-plate trailing-edge and the upstream boundary layer profiles gradually develop into a free-shear-layer profile as described by Ho and Huerre (1984). The streamwise growth of the shear-layer momentum thickness computed from the time-averaged velocity field is shown in Figure 5.3 and is compared with the experimental observations of Ho and Huang (1983) and Oster and Wygnanski (1982). The streamwise coordinate is normalized by the streamwise wavelength of the K-H vortices $\left(\lambda_{x}=0.05 L ; L=600 \mathrm{~mm}\right)$ to allow comparison with results available in the published literature. From $x / \lambda_{x}=0.0$ to 0.5 , the computed distribution has an initial streamwise gradient of momentum thickness $(d \theta / d x \approx 0.024)$ that compares favourably with the value of 0.027 predicted by the empirical correlation of Brown and Roshko (1974). Beyond the shear-layer roll-up location of $x=1.0 \lambda_{x}$ the growth rate slows down.

The computed streamwise variation of shear-layer momentum thickness beyond about $1.0 \lambda_{x}$, where the initial shear-layer roll-up occurs, is noted to compare very favourably with the experimental data of Wygnanski (1980) acquired at a comparable 


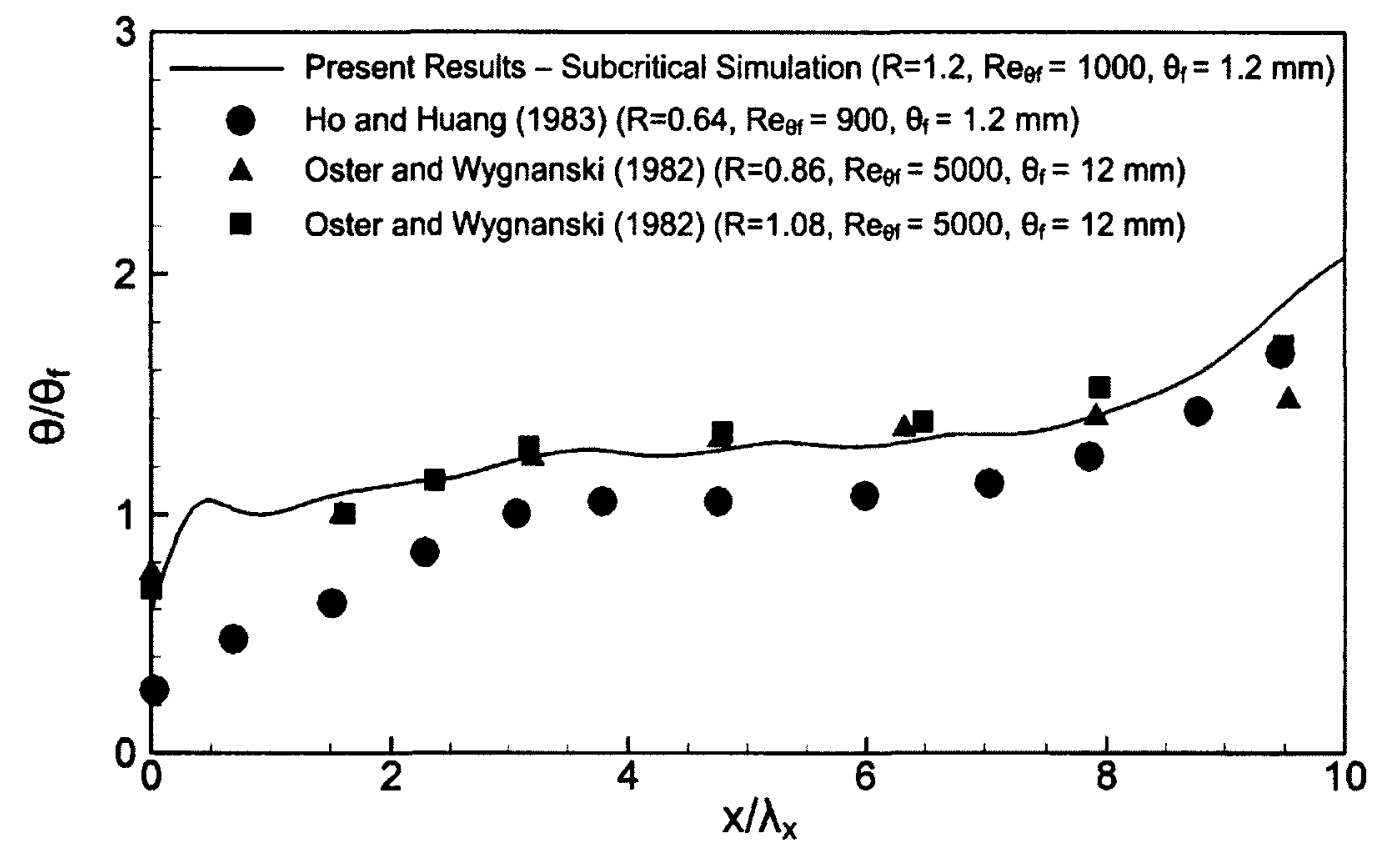

Figure 5.3: Streamwise variation of free-shear-layer momentum thickness.

velocity ratio. This serves to validate the present computations. At approximately 8.0 $\lambda_{x}$ from the trailing-edge, the primary K-H vortices formed through shear-layer roll-up begin to pair, as shown through iso-contours of spanwise vorticity in Figure 5.4. This pairing yields an increase in the effective diffusion of the time-averaged shear layer, which manifests itself as an increased streamwise rate-of-change of momentum thickness beyond $8.0 \lambda_{x}$ from the splitter-plate trailing edge, as illustrated in Figure 5.3.

\subsubsection{Primary Instability}

As is evident in Figure 5.4, the streamwise waviness caused by the inviscid (K-H) instability mode results in the roll-up of the shear layer within a streamwise distance of one wavelength of the dominant frequency of this instability. The frequency of this time-periodic roll-up process was determined in two ways: by monitoring the passage 


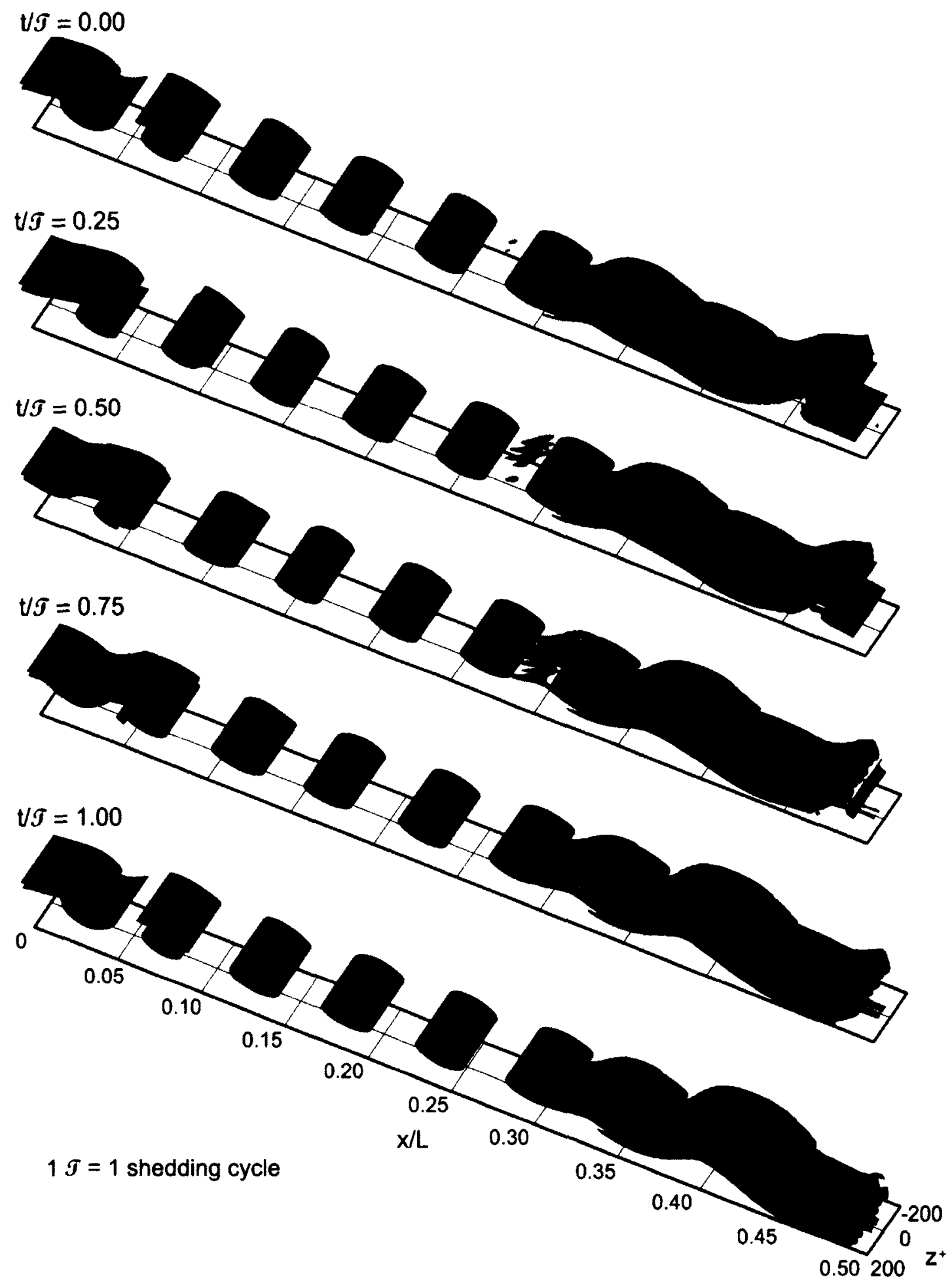

Figure 5.4: Instantaneous isocontours of spanwise and streamwise vorticity for one vortex-shedding cycle $(\mathscr{T})$ of the subcritical shear layer (green: spanwise vorticity $\left(\omega_{\mathrm{z}}=400 \mathrm{~s}^{-1}\right)$; red and blue: streamwise vorticity of counter-rotating vorticity $\left.\left(\omega_{\mathbf{x}}= \pm 40 \mathrm{~s}^{-1}\right)\right)$. 


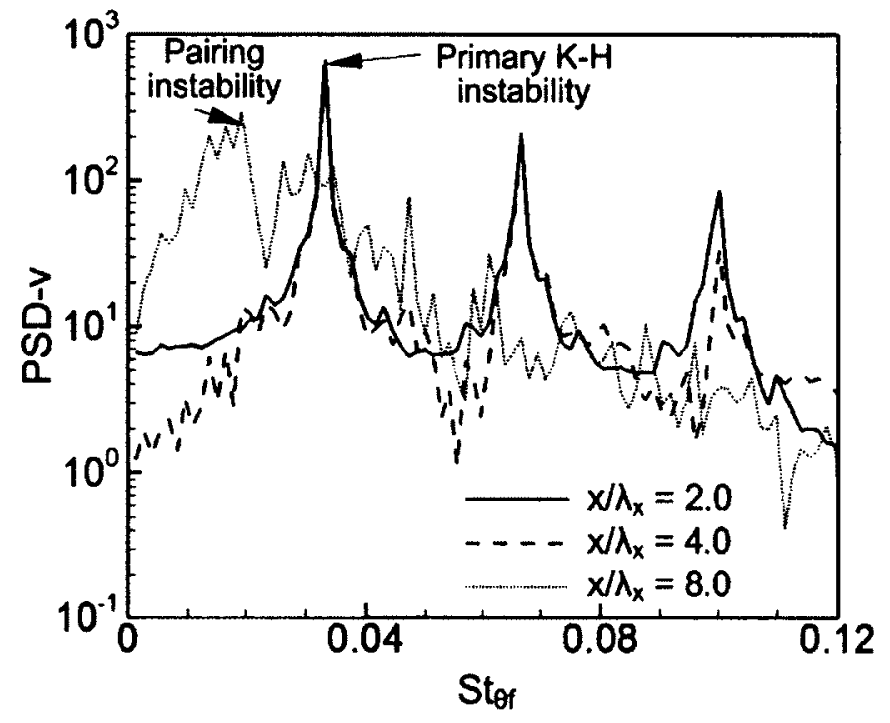

Figure 5.5: Power spectral density of $y$-component velocity fluctuation in the subcritical shear layer at $\mathrm{y} / \theta_{\mathrm{f}}=0$.

of vortices in a region of flow as described in Section 4.2 and by performing a fast Fourier transform of the $y$-component of perturbation velocity. Both methods produce a vortex-shedding Strouhal number of $S t_{\theta f}=0.032 \pm .0 .001$, which is in excellent agreement with the linear stability analysis results of Michalke (1964) for hyperbolic tangent velocity profiles. Figure 5.5 shows the power spectra of the $y$-component of perturbation velocity at three streamwise locations in the shear layer-one near the site of roll-up $\left(x / \lambda_{x}=2.0\right)$, one further downstream $\left(x / \lambda_{x}=4.0\right)$ and one near the site of pairing $\left(x / \lambda_{x}=8.0\right)$. The power spectra at $x / \lambda_{x}=2.0$ and $x / \lambda_{x}=4.0$ reveal additional peaks corresponding to higher harmonics of the K-H instability. These peaks are observed at integer multiples of the Strouhal number of vortex shedding and have also been observed in other studies of shear layers (e.g. Volino and Bohl, 2004).

As the primary K-H vortices are convected downstream, they begin to stretch and elongate in the streamwise direction. This streamwise elongation is readily visible at 


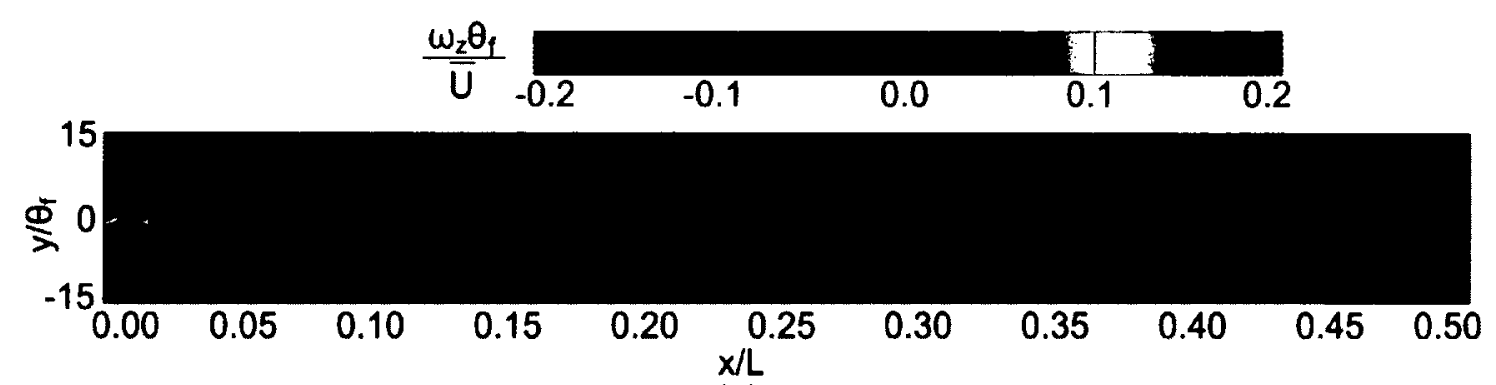

(a)

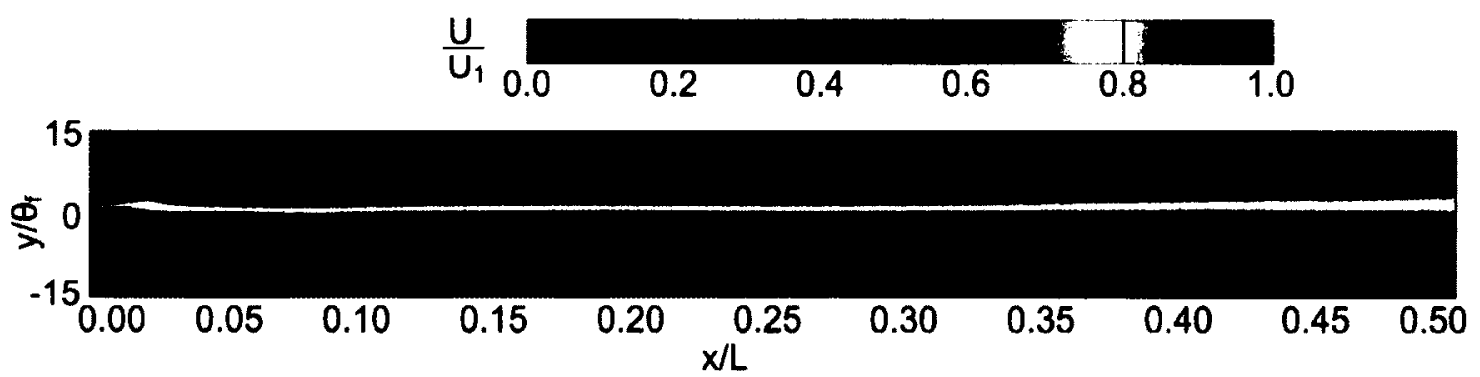

(b)

Figure 5.6: Subcritical shear layer flood plots at $\mathrm{z}^{+}=0$ of (a) instantaneous spanwise vorticity distribution and (b) time-averaged streamwise velocity distribution.

$x / L=0.32$ in Figure 5.6a, which plots the instantaneous spanwise vorticity distribution in the shear layer. The portion of the vortex exposed to the high-momentum stream above convects faster than the portion exposed to the low-momentum stream below. Consequently, the vortices experience streamwise strain that leads to their stretched appearance.

The production of the unsteady K-H vortices contributes to a thicker shear layer in a time-averaged sense, and consequently an enhanced rate of mixing between the high-momentum and low-momentum streams, as is evident in the time-averaged velocity distribution plotted in Figure 5.6b. This plot qualitatively shows that the streamwise locations beyond the initial roll-up have larger regions where the timeaveraged velocity corresponds to the mean convective velocity--shown with green colour - and thereby indicate the effectiveness of the $\mathrm{K}-\mathrm{H}$ vortices in mixing the two fluid streams. 


\subsubsection{Secondary Instability}

The wavering of the shear layer during periodic shedding of the spanwise vortices creates incongruences in the $y$-coordinate of the vortices. Consequently, an upstream vortex that is perturbed further toward the high-momentum stream convects faster than its downstream partner and it eventually overtakes it, initiating a vortex-pairing process. The power spectra of the $y$-component of perturbation velocity at the site of vortex pairing $(x / L=0.4)$, shown in Figure 5.5, reveal an additional peak at half the frequency of the dominant K-H instability frequency. This peak at $S t_{\theta f}=0.016$ corresponds to the passing frequency of the paired vortices. Pairing occurs approximately six wavelengths downstream of the site of roll-up, consistent with observations in several experimental studies at similar conditions (e.g. Ho and Huang, 1983). Figure 5.3 shows broader regions at the mean streamwise velocity over the streamwise section where vortex pairing occurs. Furthermore, Figure $5.6 \mathrm{~b}$ reveals that regions at vortex pairing have larger regions at the mean streamwise velocity. These trends suggest that pairing contributes to enhanced mixing between the high- and low-momentum streams.

Prior to the onset of the pairing instability, three-dimensional vortical structures begin to appear among the primary K-H vortices. These instabilities are observed to originate in the high-shear braids between vortices (Figure 5.4), consistent with several recent studies, including those of McAuliffe and Yaras (2007) and Brinkerhoff and Yaras (2011) that focus on separated shear layers. In Figure 5.4, the spanwise axis is presented in wall units $z^{+}=z u_{\tau} / \nu$, where $u_{\tau}$ is the friction velocity evaluated at the wall at the splitter plate trailing edge on the high-speed-stream side, and is expressed as $u_{\tau}=\left[\left.\nu(d U / d y)\right|_{w}\right]^{1 / 2}$. The choice for this unconventional scaling factor for the free shear layer is based on the assumption that the perturbations that amplify to produce the streamwise vortices originate from the upstream boundary layer as 


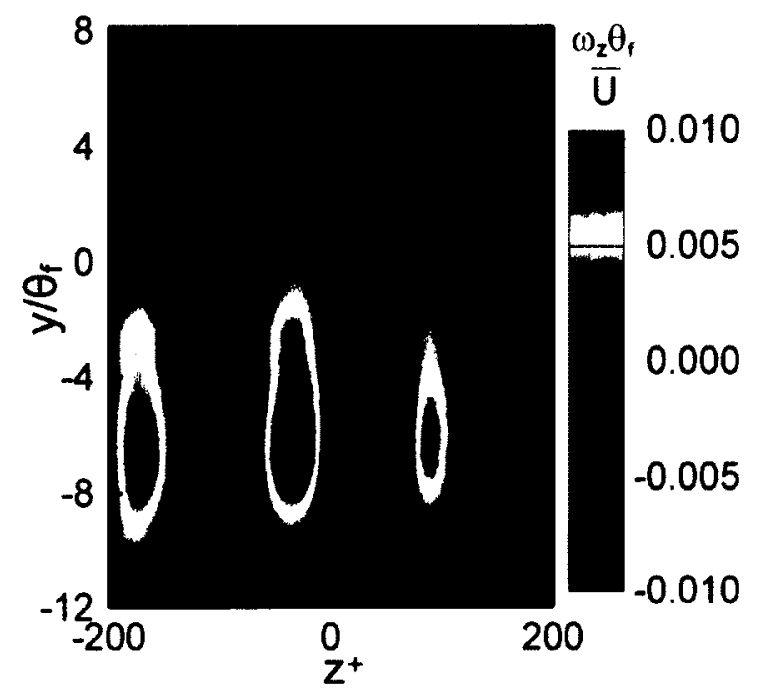

Figure 5.7: Subcritical free shear layer time-averaged streamwise vorticity flood plots in the $x=0.35 \mathrm{~L}$ plane.

noted by Brinkerhoff and Yaras (2011). The vorticity magnitudes of the streamwise vortices in Figure 5.4 are an order of magnitude smaller than that of the spanwise vortices, and, consequently, the streamwise vortices do not interact notably with the higher-vorticity-magnitude $\mathrm{K}-\mathrm{H}$ vortices. However, there are observable grooves on the spanwise K-H vortices with wavelengths corresponding to the streamwise vortices owing to the induced velocity produced by the streamwise vortices. As noted in Figure 5.7, the spanwise spacing of the streamwise vortices is $\lambda_{z}^{+}=120$.

Figure 5.8 shows the power spectra of the spanwise variation of streamwise component of perturbation velocity at several streamwise locations upstream and downstream of the splitter-plate trailing edge. The power spectra reveal that the peak corresponding to the dominant wavelength of spanwise instability in the free shear layer starts to develop in the boundary layer upstream of the trailing edge. This agrees with the results of Brinkerhoff and Yaras (2011), who suggest that the perturbations that amplify to produce three-dimensional instability in a separated shear layer originate from the viscous (T-S) instability of the upstream boundary layer. 


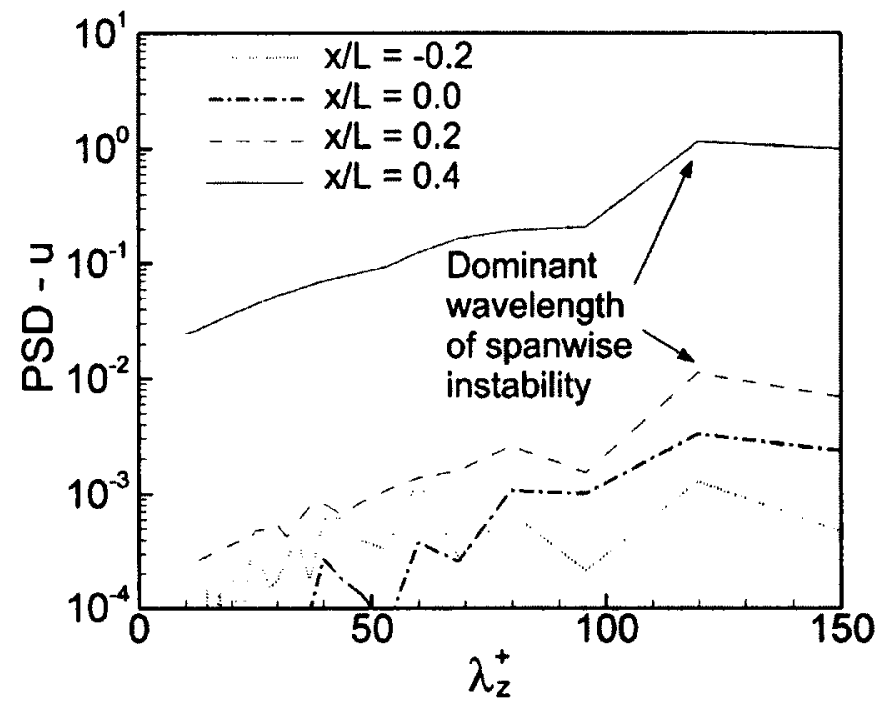

Figure 5.8: Spanwise wavenumber spectra for the subcritical shear layer at $\mathbf{y} / \theta_{\mathbf{f}}=0.3$.

\subsection{Instability Development in the Supercritical Shear Layer}

Following the subcritical shear layer simulation, a free-shear-layer simulation with heat transfer into the supercritical working fluid was performed to assess the impact of cross-stream property variation on instability development in the shear layer. Due to time constraints for performing this simulation, the time span of the statisticallysteady computed data was not sufficient to obtain reliable statistical information of the flow development. Therefore, the focus of this chapter is the transient evolution of the flow-field. The results show that the presence of cross-stream property gradients across the free shear layer alters the nature of the instability development in the shear layer. Most notably, the supercritical shear layer development includes finer spanwise vortical structures than the subcritical shear layer, with earlier, stronger and less organized development of streamwise vortices leading to earlier breakdown of the 
supercritical shear layer into small-scale turbulence. Each of these developments are discussed in turn in the following sections.

\subsubsection{Primary Instability}

The development of the supercritical free shear layer is shown through isocontours of streamwise and spanwise vorticity in Figure 5.9. The presence of spanwise rollers driven by the $\mathrm{K}-\mathrm{H}$ instability mechanism is evident. Unlike the subcritical shear layer, additional spanwise vortical structures of smaller scale are also observed. These smaller vortices are reminiscent of the flow structures that develop through the Holmboe instability mechanism (Holmboe, 1962) under the influence of gravitational force in density-stratified shear layers. This instability mechanism has been extensively studied in the context of geophysical flows, and is observed when the level of stratification is sufficiently strong as indicated by the value of the Richardson number (e.g. Lawrence et al., 1991; Lee and Claufield, 2001; Smyth, 2005; Alexakis, 2009; Carpenter et. al., 2010). The experiments of Lawrence et al. (1991) showed the co-existence of $\mathrm{K}-\mathrm{H}$ and Holmboe instability mechanisms even at low values of the Richardson number (e.g. $J \approx 0.05$ ).

Gravitational acceleration is not present in the current supercritical shear-layer simulation. However, deformation of the planar free shear layer by K-H instability results in local centripetal accelerations that appear to create conditions that are analogous to those that result in formation of Holmboe instability through the effects of gravitational forces in density-stratified shear layers. As was discussed earlier, K-H instability results in a planar shear layer assuming a streamwise-wavy pattern at the dominant wavelength of instability with accompanying streamwise curvature. The accompanying streamwise grouping of spanwise vorticity in the shear layer results in further localized deformation of the shear layer, prompting an even higher local 

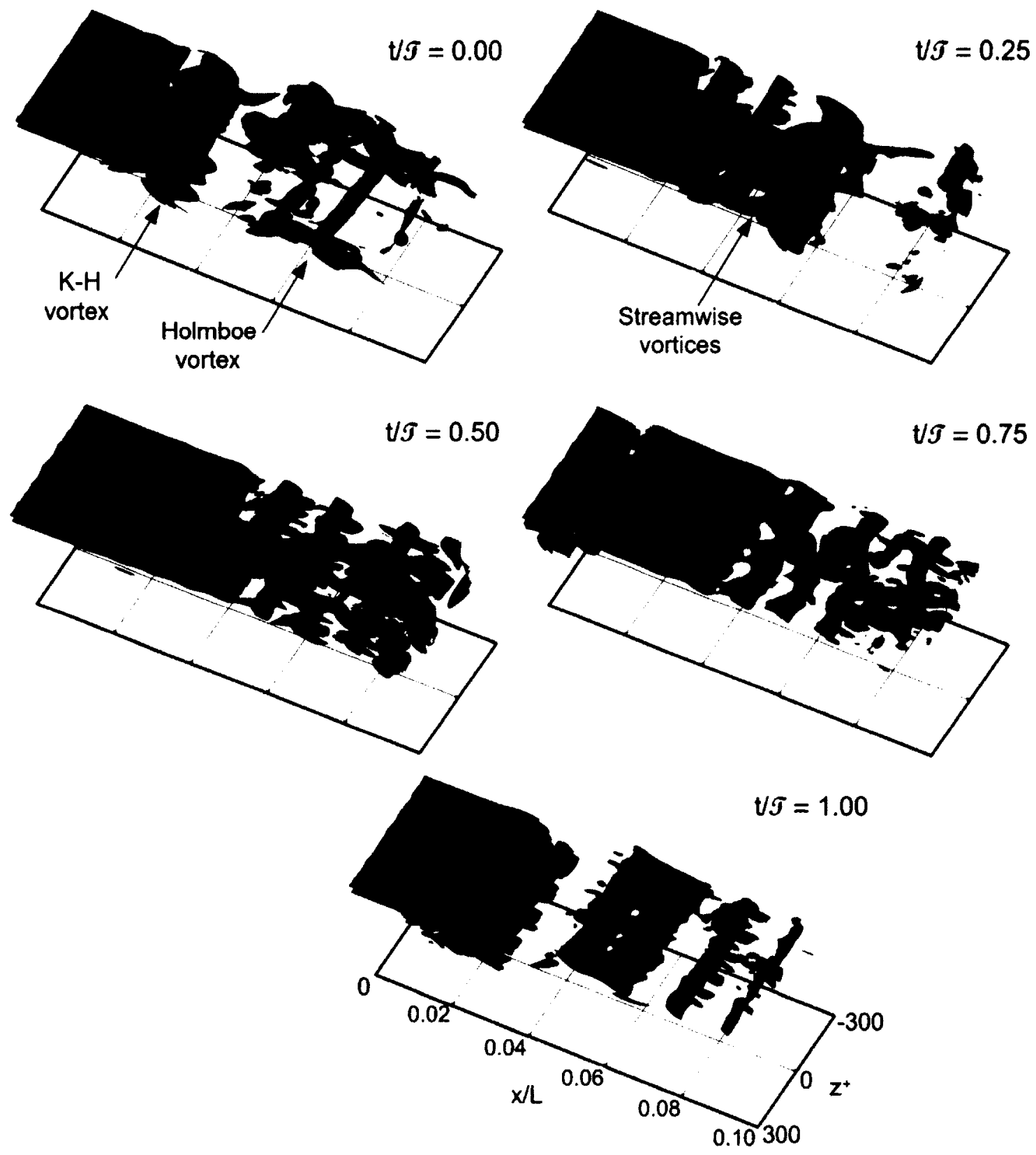

Figure 5.9: Instantaneous isocontours of spanwise and streamwise vorticity for one K-H vortex-shedding cycle for the supercritical shear layer (green: spanwise vorticity $\left(\omega_{\mathbf{z}}=20 \mathrm{~s}^{-1}\right)$; red and blue: streamwise vorticity $\left.\left(\omega_{\mathbf{x}}= \pm 20 \mathbf{s}^{-1}\right)\right)$. 
streamwise curvature that initiates the shear-layer roll-up process. These deformations of the shear layer are clearly illustrated in Figure 5.10. With the development of such streamwise curvatures, centripetal accelerations are established in the shearlayer flow field. These centripetal accelerations reach values as high as $1.0 \mathrm{~m} / \mathrm{s}^{2}$ corresponding to streamwise curvatures of about $15 \mathrm{~mm}$, resulting in local Richardson number values as high as 0.16 . As expected, the vortical structures driven by the Holmboe instability mechanism, hereafter referred to as "Holmboe vortices", form in regions of the free shear layer corresponding to this peak Richardson number value. These regions are the locations where the shear layer rolls up into coherent spanwise vortices driven by the $\mathrm{K}-\mathrm{H}$ instability mechanism. This is clearly illustrated in Figure 5.11 , where a Holmboe vortex is observed to form approximately half way through the $\mathrm{K}-\mathrm{H}$ vortex shedding cycle. A necessary condition for the growth of the Holmboe instability is that the ratio of the shear-layer thickness to the thickness over which the density varies be greater than 2.0 (Alexakis, 2005, 2007). In the present simulation, at the locations of Holmboe instability growth, this thickness ratio was observed to have a value of 5.0. Based on the streamwise spacing of the spanwise vortices observed in the flow field, with the observation in Figure 5.11 being one example, the wavenumber for the Holmboe vortices is determined to be $\alpha \delta_{\omega}=1.2$ while the $\mathrm{K}-\mathrm{H}$ vortices have a dominant wavenumber value of $\alpha \delta_{\omega}=0.8$. These wavenumber values are based on the vorticity thickness, $\delta_{\omega}$, for compatibility with the stability diagrams of Lawrence et al. (1991) that are based on shear layer height. Lawrence et al.'s stability plot for shear layers with cross-stream density gradients was presented in Chapter 2 (Figure 2.17). In that plot, wavenumbers of unstable Holmboe waves are bounded by isocontours of $\alpha c_{i}=0.25$, placing the shear layer conditions of the present case in the unstable region.

As noted in Figure 5.11, the Holmboe instability mechanism produces a spanwise vortical structure of the same sense of rotation as the larger spanwise vortex resulting 


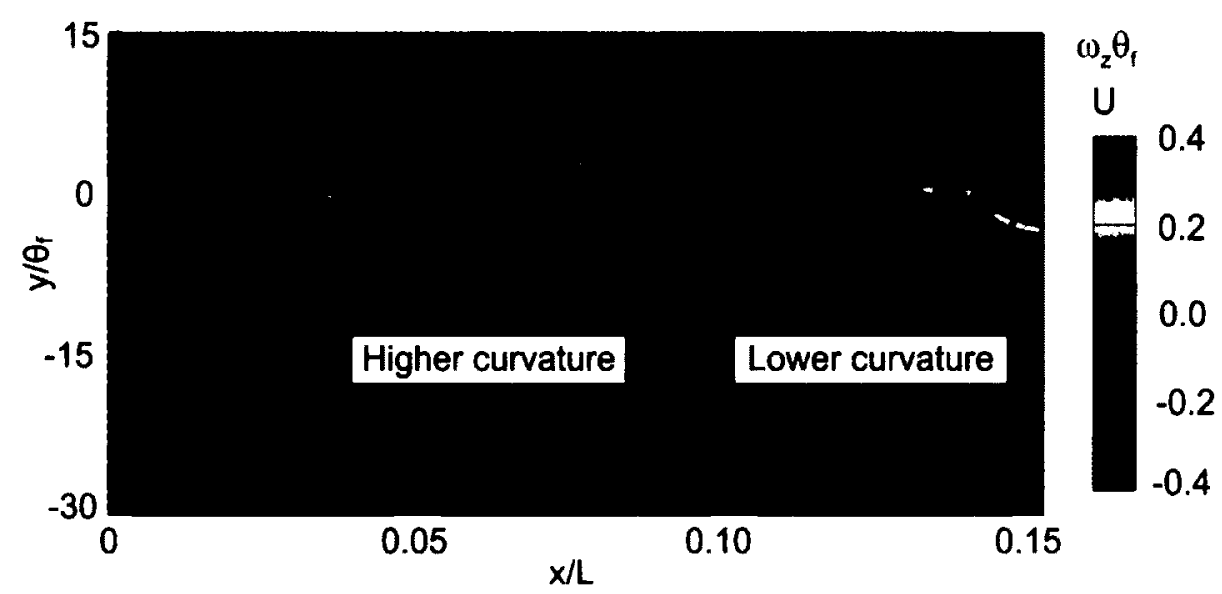

Figure 5.10: Development of streamwise curvature in the free shear layer by K-H instability.

from the K-H instability mechanism. Simultaneously, a region of opposite sign of vorticity is noted to develop between these two vortices, which is clearly evident at $t / \mathscr{T}=0.6$. Such flow dynamics is consistent with the development of the Holmboe instability waves (e.g. Carpenter et al., 2010). The flow structure associated with the region of positive (counter-clockwise) vorticity is observed to split into two, with one part convecting around the $\mathrm{K}-\mathrm{H}$ vortex, and the other convecting away from the $\mathrm{K}-\mathrm{H}$ vortex together with its counterpart of opposite vorticity under the influence of the high-momentum stream prevailing above the shear layer.

While both the K-H and Holmboe mechanisms affect the development of the flow, the mixing effect of the $\mathrm{K}-\mathrm{H}$ mechanism is expected to dominate due to the larger scale of the vortical flow structures produced by this physical mechanism. The flow pattern observed in Figure 5.9 supports this. 

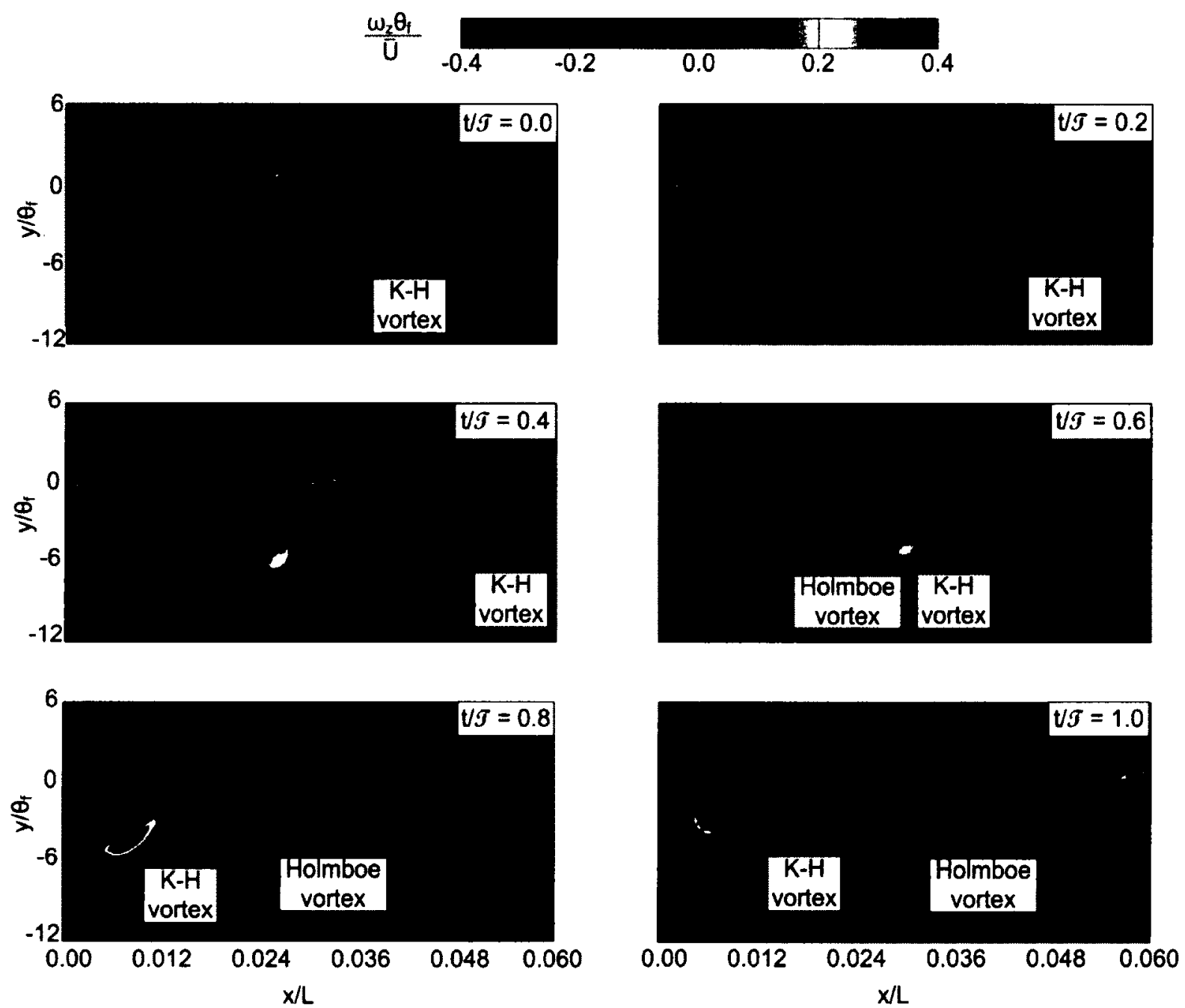

Figure 5.11: An example of the formation of $\mathrm{K}-\mathrm{H}$ and Holmboe vortices in one cycle of $\mathrm{K}-\mathrm{H}$ vortex-shedding. 


\subsubsection{Secondary Instability}

As the K-H vortices convect downstream, streamwise vortices form in the braid regions between these vortices. These streamwise vortices are noted to be more irregular and of significantly greater strength than those observed in the subcritical shear layer case. In fact, the vorticity magnitude of the spanwise and streamwise vortices are comparable as illustrated through the relative magnitudes of the vorticity isocontours in Figure 5.9. The increased number of streamwise vortices contribute to more efficient three-dimensional mixing of the shear layer. These streamwise vortices groove out undulations in the $\mathrm{K}-\mathrm{H}$ vortices, thereby commencing the process of three-dimensional, small-scale breakdown of the shear layer. The average spanwise spacing of the streamwise vortices is noted to be $\lambda_{z}^{+}=100$, which is consistent with the spacing of the streaky structures in the streamwise velocity field upstream and downstream of the splitter-plate trailing edge (Figure 5.12). The differences observed in the streak spacing is quite likely due to the mechanisms recently discovered by Azih

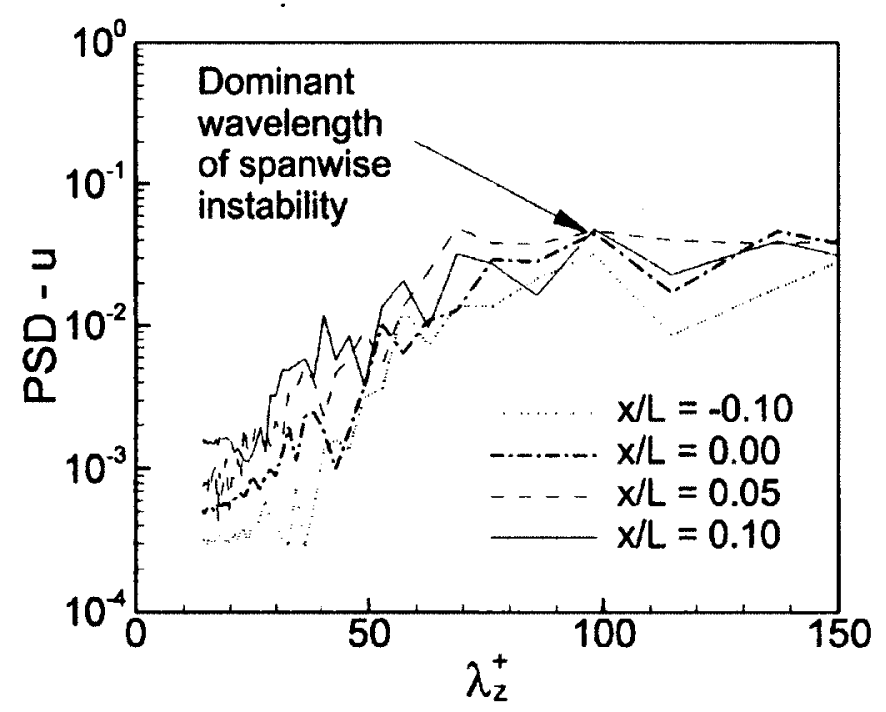

Figure 5.12: Spanwise wavenumber spectra for the supercritical shear layer at $\mathbf{y} / \theta_{\mathrm{f}}=0.3$. 
et al. $(2012 \mathrm{a}, \mathrm{b})$ in the study of heated turbulent boundary layer and channel flows in a supercritical fluid. Azih et al. discovered that large spatial gradients in viscosity and density across a shear layer can have both stabilizing and destabilizing effects on the coherent flow structures of turbulence, depending on the specific thermodynamic state and flow conditions. The present results illustrate that the mechanisms described by Azih et al. for wall-bounded, heated shear layers in supercritical fluids appear to be equally effective in free-shear layers with comparable property gradients across the shear layer. 


\section{Chapter 6}

\section{Conclusions}

This study investigates the mechanism by which proximity to a nearby wall affects the stability characteristics of a free shear layer. To accomplish this task, a twodimensional study using shear layers in close proximity to a no-slip wall is performed using a test matrix at Reynolds numbers, velocity-difference ratios and wall proximities representative of conditions in separation bubbles on airfoils. Boundary layers are developed above and below an upstream, no-slip splitter plate with a wall-normal location $\left(y_{s}\right)$ from a no-slip wall, and are merged at the trailing edge of the splitter plate to develop a separated shear layer velocity profile. This velocity profile is unstable to inviscid instabilities due to the inflection point contained in the shear layer velocity profile and, therefore, developed instability through the formation of two-dimensional Kelvin-Helmholtz (K-H) vortices. Proximity of the shear layer to the no-slip wall has a notable influence on the stability of the shear layer as a closer proximity leads to a delayed onset of vortex roll-up, delayed flow attachment onto the wall, and lower Strouhal numbers of vortex shedding. The range of Strouhal numbers observed in this study agree favourably with the range observed in previous studies of separation bubbles and free shear layers, therefore, provide a possible explanation for the large spread in Strouhal numbers found in published literature. It is observed that at lower Reynolds numbers, the shear layers have increased sensitivity to the nearby wall. A 
linear stability analysis of the flow field using the Orr-Sommerfeld equation verifies this increasing trend in the frequency of vortex shedding for increased distance from the wall. These results suggest that proximity of the shear-layer to the wall influences the relative dominance of the viscous T-S instability and inviscid K-H instability modes. Through the use of potential flow models, it is shown that a mechanism of delayed transition onset due to wall proximity is primarily through the suppression of the wall-normal motion.

Furthermore, this study investigates the effect of cross-stream property variation in supercritical water on the transition and turbulence properties of free shear layers. A direct numerical simulation (DNS) of a water shear layer with cross-stream temperature gradient is performed and compared with a DNS of a subcritical single-phase shear layer with matching Reynolds number and velocity-difference ratios. The supercritical shear layers is observed to develop an additional mechanism of instability owing to the cross-stream property gradients. Most notably, sites of streamlined curvature are observed to periodically stabilize the event of shear-layer roll-up because of the stable density field created by the radial forces acting on a fluid element as it follows a curved path. This effect is also shown to create Holmboe vortices that are weaker spanwise counter-rotating vortical-structure pairs that coexist with the $\mathrm{K}-\mathrm{H}$ vortices. The secondary three-dimensional instability of the shear layer is also modified by the supercritical state as demonstrated by the smaller spanwise-wavelength streamwise vortices compared to the subcritical case. The smaller wavelength and greater strength of these structures lead to more numerous streamwise vortices that contribute to better shear layer mixing. The behaviour of the secondary instability observed in this study suggests that instability mechanisms in supercritical fluid boundary layers observed by Azih et al. (2012) are equally applicable to free shear layer flows. 


\section{List of References}

Acarlar, M. S. and Smith, C. R. (1987). A study of hairpin vortices in a laminar boundary layer. Part 2. Hairpin vortices generated by fluid injection. Journal of Fluid Mechanics, 175:43-83.

Ackerman, J. W. (1970). Pseudoboiling heat transfer to supercritical pressurewater in smooth and ribbed tubes. Journal Heat Transfer, 92:490.

Adrian, R. J., Meihnart, C. D., and Tomkins, C. D. (2000). Vortex organization in the outer region of the turbulent boundary layer. Journal of Fluid Mechanics, $422: 1-54$

Alam, M. and Sandham, N. D. (2000). Direct numerical simulation of short laminar separation bubbles with turbulent reattachment. Journal of Fluid Mechanics, 410:1 -28 .

Alexakis, A. (2005). On Holmboes instability for smooth shear and density profiles,. Physics of Fluids, 17(084103).

Alexakis, A. (2007). Marginally unstable Holmboe modes. Physics of Fluids, 19(054105).

Alexakis, A. (2011). Stratified shear flow instabilities at large Richardson numbers. Physics of Fluids, 21(054108).

Amsden, A. A. and Harlow, F. H. (1964). Slip-instability. Physics Fluids, 7:327 34.

Anderson, J. (1995). Computational Fluid Dynamics: The Basics with Applications. McGraw-Hill, Oxford.

Azih, C., Brinkerhoff, J. R., and Yaras, M. I. (2012a). Direct numerical simulation of convective heat transfer in a zero-pressure- gradient boundary layer with supercritical water. Journal of Thermal Science, 21. 
Azih, C. and Yaras, M. I. (2012b). Direct numerical simulations of forced convection heat transfer in channels with supercritical water. The 3rd China-Canada Joint Workshop on Supercritical-Water-Cooled Reactors, CCSC-2012.

Bae, J. H. and Yoo, J. Y. (2005). Direct numerical simulation of turbulent supercritical flows with heat transfer. Physics of Fluids, 17:1 - 24.

Baig, M. F. and Chernyshenko, S. I. (2004). Regeneration mechanism of streaks in near-wall quasi-2D turbulence. European Journal of Mechanics - B/Fluids, 23:727 $-736$.

Bailey, S. C. C., Martinuzzi, R. J., and Kopp, G. A. (2002). The effects of wall proximity on vortex shedding from a square cylinder: Three-dimensional effects. Physics of Fluids, 14(12):4160 - 4177.

Bao, F. and Dallmann, U. C. (2004). Some physical aspects of separation bubble on a rounded backward-facing step. Aerospace Science and Technology, 8(2):83 91.

Batchelor, G. K. (1967). An Introduction to Fluid Dynamics. Cambridge University Press, New York.

Bayly, B. J., Orszag, S. A., and Herbert, T. (1988). Instability mechanisms in shear-flow transition. Annual Review of Fluid Mechanics, 20:359 - 391.

Bechert, D. W. and Michel, U. (1975). The control of a thin free shear layer with and without a semi-infinite plate by a pulsating flow field. Acustica, 33(5):287 307.

Bellmore, C. P. and Reid, R. L. (1983). Numerical prediction of water temperatures for near-critical para-hydrogen in turbulent upflow inside vertical tubes. Journal Heat Mass Transfer, 105.

Bernal, L. P. (1981). The coherent structure of turbulent mixing layers. I. Similarity of the primary vortex structure. II.Secondary streamwise vortex structure. PhD thesis, California Institute of Technology, Pasadena, USA.

Blazek, J. (2001). Computational Fluid Dynamics Principles and Applications. Elsevier, Oxford.

Boiko, A. V., Grek, G. R., Dovgal, A. V., and Kozlov, V. V. (2002). The origin of turbulence in near-wall flows. Springer, A2(6):937 - 948. 
Brinkerhoff, J. R. and Yaras, M. I. (2011). Interaction of viscous and inviscid instability modes in separation-bubble transition. Physics of Fluids, 23(12).

Butler, K. M. and Farrell, B. F. (1993). Optimal perturbations and streak spacing in wall-bounded turbulent shear flow. Physics of Fluids, 5:774- 777 .

Chernyshenko, S. I. and Baig, M. F. (2005). The mechanism of streak formation in near-wall turbulence. Journal of Fluid Mechanics, 544:99 - 131.

Claufield, C. P. and Peltier, W. R. (2000). The anatomy of the mixing transition in homogeneous and stratified free shear layers. Journal of Fluid Mechanics, 413:1 -47 .

Craik, A. D. D. (1971). Non-linear resonant instability in boundary layers. Journal of Fluid Mechanics, 50:93 - 413.

Craik, A. D. D. (1980). Nonlinear evolution and breakdown in unstable boundary layers. Journal of Fluid Mechanics, 99:247 - 265.

Crouch, J. D. (1991). Localized receptivity of boundary layers. Physics Fluids, A4:1408 - 14 .

Curtis, E. M., Hodson, H. P., Banieghbal, M. R., Denton, J. D., Howell, R. J., and Harvey, N. W. (1997). Development of blade profiles for low-pressure turbine applications journal of turbomachinery. Journal of Turbomachinery, 119:531 538 .

Das, D. (1998). Evolution and instability of unsteady boundary-layers with reverse flow. PhD thesis Department of Mechanical Engineering, Indian Institute of Science, Bangalore, India, A2(6):937 - 948.

Diwan, S. S. and Ramesh, O. N. (2009). On the origin of the inflectional instability of a laminar separation bubble. Journal of Fluid Mechanics, 629:263 - 298.

Doligalski, T. L., Smith, C. R., and Walker, J. D. A. (1994). Vortex interactions with walls. Annual Review of Fluid Mechanics, 26(2):573 - 616.

Dovgal, A. V., Kozlov, V. V., and Michalke, A. (1994). Laminar boundary layer separation: Instability and associated phenomena. Progress in Aerospace Sciences, $30: 61-94$.

Drazin, P. G. and Reid, W. H. (1981). Hydrodynamic Stability. Cambridge Press, Cambridge. 
Emelyanov, V. M., Gorbunov, A. A., Lednev, A. K., and Nikitin, S. A. The special features of heat transfer in a supercritical fluid: Mathematical and physical modeling results. Institute for Problems of Mechanics, Russian Academy of Sciences, pr. Vernadskogo 101, Moscows, 117526 Russia.

Emmons, H. W. (1951). The laminar-turbulent transition in a boundary layer-Part 1. Journal of Aeronautical Sciences, 18:490 - 498.

Erdogan, K., Pablo, D., and Cor, P. (1993). Supercritical fluids: Fundamentals and application. NATO Science Series.

Estevadeordal, J. and Kleis, S. J. (1999). High resolution measurements of twodimensional instabilities and turbulence transition in plane mixing layers. Experiments in Fluids, 27:378 - 390.

Fjortoft, R. (1949). Application of integral theorems in deriving criteria of stability for laminar flows and for the baroclinic circular vortex. Geofysiske Publikasjoner, $17: 1-52$.

Fraser, C. J., Milne, J. S., and Gardiner, I. D. (1988). A combined visual and hot-wire anemometer investigation of boundary layer transition. Proc. Inst. Mech. Eng., 202:195 - 203.

Freymuth, P. (1966). On transition in a separated laminar boundary layer. Journal Fluid Mechanics, 25:683 - 704.

Goldstein, S. (1931). On the stability of superposed streams of fluids of different densities. Philosophical Transactions of the Royal Society, 132:524 - 548.

Gostelow, J. P. and Thomas, R. L. (1992). Interactions between propagating wakes and flow instabilities in the presence of a laminar separation bubble. ASME Paper No. 2006-GT-91193.

Granville, P. S. (1953). The calculation of the viscous drag of bodies of revolution. David Taylor Model Basin Report, page 849.

Grek, G. R., Kozlov, V. V., Katasonov, M. M., and Chernoral, M. M. (2000). Experimental study of a lambda-structure development and its transformation into the turbulent spot. Current Science, 79:781 - 790.

Haidari, H. A. and Smith, C. R. (1994). The generation and regeneration of single hairpin vortices. Journal Fluid Mechanics, 277:135 - 162. 
Hall, W. B. (1971). Heat transfer near the critical points. Advances in Heat Transfer, Academic Press, New York., 6.

Hall, W. B. and Jackson, J. D. (1969). Laminarization of a turbulent pipe flow by buoyancy forces. ASME Paper No. 69-HT-55, ASME 11th National Heat Transfer Conference, Minneapolis, MN ASME, New York,.

Hammond, D. A. and Redekopp, L. G. (1998). Local and global instability properties of separation bubbles. European Journal of Mechanics - B/Fluids, 17(2):145 -164 .

Hansen, G. and Owen, S. (2008). Mesh generation technology for nuclear reactor simulation; barriers and opportunities. Nuclear Engineering and Design, 238:2590 -2605 .

Hatman, H. and Wang, T. (1999). A prediction model for separated-flow transition. Journal of Turbomachinery, 121(3):594 - 602.

Hauptmann, E. G. (1967). The infuence of temperature dependent viscosity on laminar boundary-layer stability. International Journal Heat Mass Transfer, 1:1049 1052 .

He, S., Kim, W. S., Jiang, P. X., and Jackson, J. D. (2004). Simulation of mixed convection heat transfer to carbon dioxide at supercritical pressure. Journal Fluid Mechanics, 218.

Heinrich, R. A. and Kerschen, E. J. (1989). Leading-edge boundary-layer receptivity to free-stream disturbance structures. AIAA, 69:596.

Herbert, T. (1988). Secondary instability of boundary layers. Annual Review of Fluid Mechanics, 20:487 - 526.

Herwig, H. and Schäfer, P. (1992). Influence of variable properties on the stability of two-dimensional boundary layers. Journal Fluid Mechanics, 243:1 - 14.

Ho, C. M. and Huang, L. S. (1982). Subharmonics and vortex merging in mixing layers. Journal of Fluid Mechanics, 119:443 - 73.

Ho, C. M. and Huerre, P. (1984). Perturbed free shear layers. Annual Review of Fluid Mechanics, 16:365 - 424.

Holmboe, J. (1962). On the behaviour of symmetric waves in stratified shear layers. Geofysiske Publikasjoner, 24(67). 
Hourmouziadis, J. (1989). Aerodynamic design of low pressure turbines . Blading Design for Axial Turbomachines, AGARD Lecture Series, 167.

Howell, J. R. and Lee, S. H. (1999). Convective heat transfer in the entrance region of a vertical tube for water near the thermodynamic critical point. International Journal Heat Mass Transfer, 42.

Jackson, J. D. and Hall, W. B. (1979). Turbulent forced convection in channels and bundles. Kaka, S., Spalding, D.B. (Eds.) Hemisphere Publishing Corp. New York, USA, 2:563 612.

Joong, B., Jung, Y., and Haecheon, C. (1970). Direct numerical simulation of turbulent supercritical flows with heat transfer. Physics of Fluids, 17.

Kalitzin, G. (2003). DNS of fully turbulent flow in a LPT passage. International Journal of Heat and Fluid Flow, 24:636 - 644 .

Klebanoff, P. S. and Tidstrom, K. D. (1959). Evolution of amplified waves leading to transition in a boundary layer with zero pressure gradient. National Advisory Committee for Aeronautics Report No. D195.

Klebanoff, P. S. and Tidstrom, K. D. (1972). Mechanism by which a twodimensional roughness element induces boundary layer transition. Physics of Fluids, $15: 1173-1788$.

Knapp, C. F. and Roache, P. J. (1968). A combined visual and hot-wire anemometer investigation of boundary layer transition. A.I.A.A., 6:29-36.

Konrad, J. H. (1976). An experimental investigation of mixing in two-dimensional turbulent shear flows with applications to diffusion-limited chemical reactions. $\mathrm{PhD}$ thesis, California Institute Technology, USA.

Koshizuka, S., Takano, N., Oka, Y., and Jackson, J. D. (1995). Numerical analysis of deterioration phenomena in heat transfer to supercritical water. International Journal Heat Mass Transfer, 38.

Kundu, P. K., Cohen, I. M., and Hu, H. H. (2004). Fluid Mechanics (3rd edition). Academic Press, New York.

Lawrence, G. A., Browand, F. K., and Redekopp, L. G. (1991). The stability of a sheared density interface. Physics of Fluids, A3-(12):2360 - 2370. 
Lee, V. and Claufield, C. P. (2001). Nonlinear evolution of a layered stratified shear flow. Dynamics of Atmospheres and Oceans, 34:103 - 124.

Leon, S. B. and Askan, N. (2010). Improving the understanding of thermalhydraulics and heat transfer for super critical water cooled reactors. International Atomic Energy Agency Proceedings of ICAPP 10.

Liepmann, H. W. and Fila, G. H. (1947). Investigations of effects of surface temperature and single roughness elements on boundary-layer transition. NACA, 890 .

Lin, S. J. and Corcos, G. M. (1983). The mixing layer: Deterministic models of a turbulentflow. part iii:the effect of plane strain on the dynamics of streamwise vortices. Journal Fluid Mechanics, 141:139 - 178.

Mack, L. M. (1984a). Boundary layer linear stability theory. Jet Propulsion Lab., Document ID: 19840025688, Pasadena, Calif.

Mack, L. M. (1984b). Transition prediction and linear stability theory. AGARD Report No. 709 .

Maslowe, S. A. (1977a). Weakly nonlinear stability of a viscous free shear layer. Journal of Fluid Mechanics, 79:689 - 702.

Mayle, R. E. (1991). The role of laminar-turbulent transition in gas turbine engines. Journal of Turbomachinery, 113:509 - 536 .

McAuliffe, B. R. and Yaras, M. I. (2005). Separation-bubble-transition measurements on a low-Re airfoil using particle image velocimetry. ASME Paper No. GT2005-68663.

McAuliffe, B. R. and Yaras, M. I. (2007a). Numerical study of turbulent spot development in a separated shear layer. ASME Paper No. 2007-GT-27064.

McAuliffe, B. R. and Yaras, M. I. (2007b). Transition mechanisms in separation bubbles under low and elevated freestream turbulence. ASME Paper No. GT200\%27605 .

Michalke, A. (1972). The instability of free shear layers. Progress in Aerospace Sciences, 12:213- 39 .

Michelassi, V., Wissink, J., and Rodi, W. (2002). Analysis of DNS and LES of flow in a low pressure turbine cascade with incoming wakes and comparison with experiments. Flow, Turbulence and Combustion, 69:295 - 330 . 
Miller, R. S., Kenneth, G. H., and Bellan, J. D. (2001). Direct numerical simulations of supercritical fluid mixing layers applied to heptane-nitrogen. Journal Fluid Mechanics, 436.

Moon, H. T. and Weidman, P. D. (1988). Local vortex pairing in a free shear layer. Physics Fluids, 31.

Morkovin, M. V. (1969). On the Many Faces of Transition, pages 1-31. Viscous Drag Reduction. Plenum.

Morkovin, M. V. (1993). Bypass-transition research: Issues and philosophy. In Ashpis, D. E., Gatski, T. B., and Hirsh, R., editors, Instabilities and Turbulence in Engineering Flows, pages 3 - 30. Kluwer Academic Publishers, Boston.

Muti-Lin, J. C. and Pauley, L. L. (1996). Low-Reynolds- number separation on an airfoil. AIAA Journal, 34:1570 - 1577.

Na, Y. and Moin, P. (1998). Direct numerical simulation of a separated turbulent boundary layer. Journal of Fluid Mechanics, 374:379 - 405.

Nayfeh, A. H., Ragab, A. A., and Al-Maaitah, A. A. (1988). Effect of bulges on the stability of boundary layers. AIAA Journal, 31(2):796.

Nayfeh, A. H., Ragab, S. A., and Masad, J. A. (1990). Effect of a bulge on the subharmonic instability of boundary layers. Physics Fluids, A2(6):937 - 948.

Ni, J. H. and Armaly, B. F. (2002). Reattachment of three-dimensional separated flow adjacent to backward-facing step. Proceedings of the AIAA/ASME Joint Thermophysics and Heat Transfer Conference, AIAA Paper No. 3013, St. Louis, MO, June 24 28, 30:61 - 94.

Obremski, H. J., Morkovin, M. V., and Landahl, M. (1969). A portfolio of stability characteristics of incompressible boundary layers. NATO AGARD, (134):128.

Okongo, N. and Bellan, J. (2002). Direct numerical simulation of a transitional supercritical binary mixing layer: heptane and nitrogen. J Fluid Mech, 464:1 - 34 .

Orr, W. M. (1907). The stability or instability of the steady motions of a liquid. Proceedings of the Royal Irish Academy, 27:9-138.

Oster, D. and Wygnanski, I. (1982). The forced mixing layer between parallel streams. Journal Fluid Mechanics, 123:123:91 - 130. 
Ozgen, S. A. (2004). Effect of heat transfer on stability and transition characteristics of boundary-layers. International Journal of Heat and Mass Transfer, 47:4697 -4712 .

Pierrehumbert, R. T. and Widnall, S. E. (1982). The two- and three-dimensional instabilities of a spatiaily periodic shear layer. Journal Fluid Mechanics, 114:59 82.

Pioro, I. L., Khartabil, H. F., and Duffey, R. B. (2004). Heat transfer to supercritical fluids flowing in channels - empirical correlations(survey). Nuclear Engineering and Design, 230:69 - 91 .

Praisner, T. J. and Clark, J. P. (2007). Predicting transition in turbomachinerypart 1: A review and new model development. Journal of Turbomachinery, 129:1 -13 .

Prandtl, L. (1905). Early developments of modern aerodynamics. Internationalen Mathematiker-Kongresses in Heidelberg, page 77.

Rayleigh, L. (1880). On the stability or instability of certain fluid motions. Philosophical Transactions of the Royal Society, 11:57 - 70.

Reed, H., Saric, W. S., and Arnal, D. (1996). Linear stability theory applied to boundary layers. Annual Review of Fluid Mechanics, 28:389 - 428.

Reed, H. L. and H., N. A. (1986). Numerical-perturbation technique for stability of flat-plate boundary layers with suction. AIAA Journal, 24:208-214.

Reshotko, E. (2001). Transient growth: A factor in bypass transition. Physics Fluids, 13:1067 - 75 .

Reynolds, G. A. and Saric, W. S. (1986). Transitional flow on axial turbomachine blading. AIAA Journal, 27(2):595-602.

Rist, U. and Augustin, K. (2006). Control of laminar separation bubbles using instability waves. AIAA Journal, 44:2217-2223.

Rist, U. and Maucher, U. (2002). Investigations of time- growing instabilities in laminar separation bubbles. European Journal of Mechanics, B/Fluids, 21:495 509.

Roberts, S. K. and Yaras, M. I. (2005a). Modeling transition in separated and attached boundary layers. Journal of Turbomachinery, 127:402 - 411. 
Roberts, S. K. and Yaras, M. I. (2005b). Modeling transition in separated and attached boundary layers. ASME Journal of Turbomachinery, 127:402 - 411 .

Roelof, F. (2005). CFD Analysis of Heat Transfer to Supercritical Water Flowing Vertically Upward in a Tube.

Sandham, N. D. and Adams, N. A. (1993). Numerical-simulation of boundarylayer-transition at mach 2. Applied Scientific Research, 51:371 - 375.

Saric, W. S., Reed, H. L., and Kerschen, E. J. (2002). Boundary-layer receptivity to freestream disturbances. Annual Review of Fluid Mechanics, 34:291 - 319.

Schlichting, H. (1968). Boundary Layer Theory. McGraw-Hill, New York.

Schmid, P. J. and Henningson, D. S. (2001). Stability and Transition in Shear Flows. Springer-Verlag, Berlin.

Schroder, A. and Kompenhans, J. (2004). Investigation of a turbulent spot using multi-plane stereo particle image velocimetry. Experiments in Fluids, 36:82-90.

Schubauer, G. B. and Skramstad, H. K. (1947). Laminar boundary-layer oscillations and transition on a fiat plate. Journal of research of the National Bureau of Standards, 38:251.

Singer, B. A. and Joslin, R. D. (1994). Metamorphosis of a hairpin vortex into a young turbulent spot. Physics of Fluids, 6(6):3724 - 3736 .

Smith, C. R., Walker, J. D. A., Haidari, A. H., and Sobrun, U. (1991). On the dynamics of near-wall turbulence. Philosophical Transactions of the Royal Society, 336:131 - 175 .

Smyth, W. D. (2006). Secondary circulations in Holmboe waves. Physics of Fluids, 18(6)(064104).

Smyth, W. D. and Peltier, W. R. (1989). The transition between Kelvin-Helmholtz and Holmboe instability: An investigation of the over-reflection hypothesis. $D y$ namics of Atmospheres and Oceans, 46.

Sommerfeld, A. (1908). Ein beitrag zur hydrodynamische erklärung der turbulenten flüssigkeitsbewegungen. Proceedings of the 4th International Congress of Mathematicians, 3:116 - 124 .

Spalart, P. R. and Strelets, M. K. (2000). Mechanism of transition and heat transfer in a separation bubble. Journal of Fluid Mechanics, 403:329 - 349. 
Spang, B. (2011). Thermodynamic and transport properties of water and steam.

Suponitsky, V., Cohen, J., and Bar-Yoseph, P. Z. (2005). The generation of streaks and hairpin vortices from a localized vortex disturbance embedded in unbounded uniform shear flow. Journal of Fluid Mechanics, 535(-1):65 - 100.

Taghavi, H. and Wazzan, A. R. (1974a). Spatial stability of some falkner-skan profiles with reversed flow. Physics of Fluid, 17(12):2181 - 2183.

Taghavi, H. and Wazzan, A. R. (1974b). Spatial stability of some falknerskan profiles with reversed flow. Physics Fluids, 17(12):2181 - 2183.

Talan, M. and Hourmouziadis, J. (2002). Characteristic regimes of transitional separation bubbles in unsteady flow. Flow, Turbulence and Combustion, 69:207 227.

Tani, I. (1977). History of boundary layer theory. Ann. Rev. of Fluid Mech., 9:87 -111 .

Taylor, G. I. (1931). Effect of variation in density on the stability of superposed streams of fluid. Philosophical Transactions of the Royal Society, 132:499-523.

Tollmien, W. (1908). Ein beitrag zur hydrodynamische erklärung der turbulenten flüssigkeitsbewegungen. Proceedings of the 4th International Congress of Mathematicians, 3:116124.

Tour, C. C. (1822). Effect of a bulge on the subharmonic instability of boundary layers. Ann. Chem. Phys, 2:127.

Volino, R. J. and Bohl, D. G. (2004). Separated flow transition mechanism and prediction with high and low freestream turbulence under low pressure turbine conditions. ASME Paper No. 2004-GT-53360.

Voltchanov, H. and Yaras, M. I. (2012). Effect of forcing free shear layer using spanwise waviness.

Wagner, W. and Prub, A. (2002). The iapws formulation 1995 for the thermodynamic properties of ordinary water substance for general and scientific use. Journal Physical Chemistry, 31:387 - 535.

Walker, G. J. (1993). The role of laminar-turbulent transition in gas turbine engines: A discussion. Journal of Turbomachinery, 115(2):207 - 216. 
Walker, G. J. and Gostelow, J. P. (1989). Effects of adverse pressure gradients on the nature and length of boundary layer transition. Journal of Turbomachinery, $89: 274$.

Watmuff, J. H. (1997). Interactions between klebanoff modes and ts waves in a blasius boundary layer. AIAA Paper no. 97-0558.

Wazzan, A. R., Taghavi, H., and Pafford, D. (1986). Spatial viscous instability of the incompressible falkner-skan similarity profile at separation. Physics of Fluids, 29(7):2039 - 2041.

White, F. M. (1991). Viscous Fluid Flow. McGraw-Hill, New York.

Wille, R. (1963). Beitrige zur Phinomenologie der Freistrahlen. Z. Fluwiss, 33:11 -222 .

Williamson, C. H. K. (1992). The natural and forced formation of spot-like vortex dislocations in the transition of a wake. Journal of Fluid Mechanics, 243:393.

Winant, C. D. and Browand, F. K. (1974). Vortex pairing: The mechanism of turbulent mixing-layer growth at moderate Reynolds number. Journal of Fluid Mechanics, 63:237 - 255 .

Wygnanski, I., Haritonidis, J. H., and Zilberman, H. (1982). On the spreading of a turbulent spot in the absence of a pressure gradient. Journal Fluid Mechanics, 123:69- 90 .

Wygnanski, I., Sokolov, M., and Friedman, D. (1976). On a turbulent 'spot' in a laminar boundary layer. Journal of Fluid Mechanics, 78:785 - 819 .

Yamagata, K., Nishikawa, K., and Hasegawa, S. (1972). Forced convective heat transfer to supercritical water flowing in tubes. International Journal Heat Mass Transfer, 15 (12):2575 2593.

Yan, J. and Karoutas, Z. (2011). Boost in computing power stoking a nuclear revival. Visited: September, 2012. <<http://ascr-discovery.science.doe.gov/ genealogy/exa_nucl_energy1.shtml > .

Yang, Z. and Voke, P. R. (2001). Large-eddy simulation of boundary-layer separation and transition at a change of surface curvature. Journal of Fluid Mechanics, 439:305 - 333 . 
Yaras, M. I. (2007). An experimental study of artificially-generated turbulent spots under strong favorable pressure gradients and freestream turbulence. Journal of Fluids Engineering, 129:563 - 572. 


\section{Appendix A}

\section{Supercritical Water Thermodynamic}

\section{Table Validation}

Due to the large gradients in fluid properties near the critical point, the creation of an adequate look-up table for the thermophysical properties of supercritical water presents a challenge because of the trade-off between reasonable computing times and the accuracy of the simulated results. Therefore, this Appendix provides supplementary information on the creation of a look-up table that is able to accurately resolve the variation in fluid properties of supercritical water near the critical point. Specifically, Section A.2 introduces the NIST and IAPWS databases, two prominent databases for the properties of water, from which the water properties were obtained. Section A.3 and A.4 examines the formulation of water properties following the IAPWS standard and compares the NIST formulation with the IAPWS-97 formulation. Section A.5 describes the procedure used to select an appropriate temperature and pressure increment for the look-up table used in this study. Finally, Section A.6 validates the look-up table using a thermodynamic grid convergence study. 


\section{A.1 NIST and IAPWS Water Property Databases}

The International Association for the Properties of Water and Steam (IAPWS) represents an international organization consisting of scientists from over 25 countries whose mandate is to provide internationally-accepted formulations of the thermodynamic properties of water and steam within an extensive range. In 1995, IAPWS published IAPWS Formulation 1995 for the Thermodynamic Properties of Ordinary Water Substance for Scientific and General Use "IAPWS-95" (IAPWS, 2009), which constitutes the most-accurate formulation of water properties available for scientific use. For this reason, the National Institute of Standards and Technology (NIST) adopts this standard and provides data available in the form of a table indexed by temperature and pressure (NIST, 2011). However, the 1995 IAPWS formulation requires considerable computational effort to solve and significantly increased the computational time required for industrial and engineering applications. Consequently, in 1997, IAPWS released IAPWS Industrial Formulation 1997 for the Thermodynamic Properties of Water and Steam, "IAPWS-97" (IAPWS, 2007) which provide formulations that may be numerically solved with speeds that surpass the IAPWS-95 formulation and satisfy the special need for consistency in the steam power industry (IAPWS, 2009). This study evaluates water properties under the IAPWS-97 standard using the Visual Basic code developed by Spang (2002). Since the NIST database that is composed of the IAPWS-95 standard and the IAPWS-97 standard are both commonly used to obtain water data, it is important to verify the agreement between the two databases within the thermodynamic property range of the current study to adequately select a database. Furthermore ANSYS CFX implements the IAPWS -97 formulations and the validity of this formulation for the relevant thermodynamic and transport properties involved in heat transfer and transition should be assessed to justify the selection of the industrial formulation as opposed implementing the 
IAPWS-95 properties as a user-defined function within the ANSYS CFX solver.

\section{A.2 IAPWS Water Property Formulations}

IAPWS-97 formulates water properties by partitioning the property space into five thermodynamic regions as shown schematically in Figure A.1. Within each region, a "basic equation" for free energy is supplied that is validated by flow calorimetry measurements by Wagner (2008). Regions 1,2 and 5 are characterized by a basic equation in terms of Gibbs free energy. The region above the critical point, region 3 , is characterized by a basic equation in terms of Helmholtz free energy. All relevant thermodynamic properties including density $(\rho)$, specific enthalpy $(h)$, specific entropy $(s)$, and isobaric heat capacity $\left(c_{p}\right)$, can be calculated from these relations. Region 4, the two phase region, is governed by a saturation pressure equation. The region of interest in the present study is the supercritical region which mainly consists of region 3. The basic equation for this region is given in Equation A.1.

$$
\frac{f_{3}(\rho, T)}{R T}=\phi(\delta, \tau)=n_{1} \ln \delta+\sum_{i=2}^{40} n_{i} \delta^{I_{i}} \tau^{J_{i}}
$$

Here the $\delta$ and $\tau$ represent non-dimensional density $\left(\rho / \rho_{c}\right)$ and temperature $\left(T / T_{c}\right)$ respectively. The non-dimensional free energy is represented by $\phi$. Constants $n_{i}, I_{i}$, and $J_{i}$ are the polynomial coefficients, density exponents and temperature exponents respectively for the IAPWS-97 correlation for region 3, shown in Table A.1. The above expression provides a relation for the Helmoltz free energy function for energy function region $3\left(f_{3}(\rho, T)\right)$ that may be used with the thermodynamic identities given in Table A.2 to find pressure, specific enthalpy, isobaric heat capacity and specific entropy. 


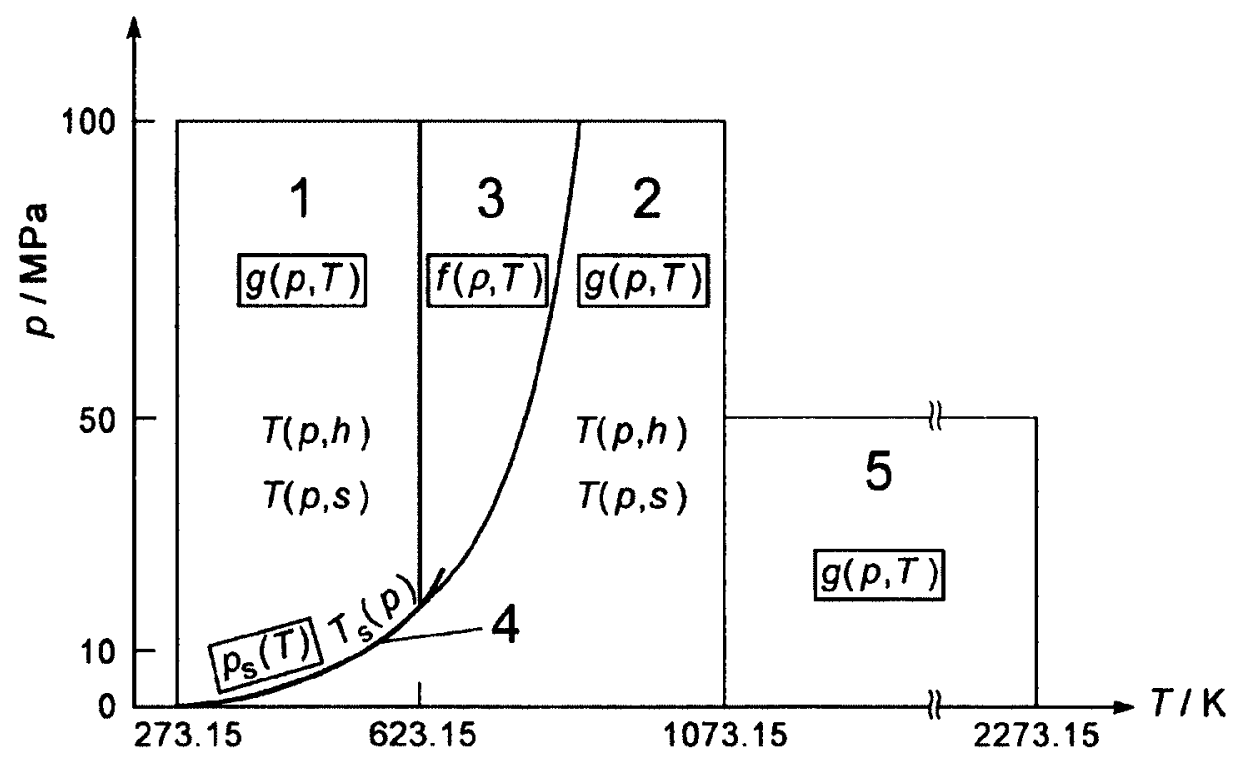

Figure A.1: Comparison of different fluids near the critical point(IAPWS, 2007)

\section{Heat Transfer and Transport Properties}

The thermal conductivity and viscosity are described in the IAPWS-95 and IAPWS97 releases, respectively. A simplified model for the correlation used to find dynamic viscosity is shown in Equation A.2, where the net viscosity is determined by the contributions due to terms $\bar{\mu}_{0}, \mu_{1}, \mu_{2}$. The base viscosity, corresponding to the dilute gas limit is represented by the first term $\bar{\mu}_{0}$. The second term $\left(\mu_{1}\right)$ represents the contributions due to the finite density of the fluid. Finally, $\mu_{2}$ represents the enhanced viscosity due to the non-linear behaviour near the critical point. The details of the formulations are described in Release on the IAPWS Formulation 2008 for the Viscosity of Ordinary Water Substance(IAPWS 1998a).

$$
\bar{\mu}=\overline{\mu_{0}}(T) \times \mu_{1}(T, \rho) \times \mu_{2}(T, \rho)
$$

Correlations for thermal conductivity takes a similar form to viscosity and is shown 
Table A.1: Coefficients and exponents of the basic equation $\mathbf{f}_{\mathbf{3}}(\rho, \mathbf{T})$ in its dimensionless form (IAPWS, 2007)

\begin{tabular}{llllllll}
\hline$i$ & $I_{i}$ & $J_{i}$ & $n_{i}$ & $i$ & $I_{i}$ & $J_{i}$ & $n_{i}$ \\
\hline 1 & - & - & $0.10658070028513 \times 10^{1}$ & 21 & 3 & 4 & $0.20189915023570 \times 10^{1}$ \\
2 & 0 & 0 & $0.15732845290239 \times 10^{2}$ & 22 & 3 & 16 & $0.82147637173963 \times 10^{-2}$ \\
3 & 0 & 1 & $0.20944396974307 \times 10^{2}$ & 23 & 3 & 26 & 0.47596035734923 \\
4 & 0 & 2 & $0.76867707878716 \times 10^{1}$ & 24 & 4 & 0 & $0.43984074473500 \times 10^{-1}$ \\
5 & 0 & 7 & $0.26185947787954 \times 10^{1}$ & 25 & 4 & 2 & 0.44476435428739 \\
6 & 0 & 10 & $0.28080781148620 \times 10^{1}$ & 26 & 4 & 4 & 0.90572070719733 \\
7 & 0 & 12 & $0.12053369696517 \times 10^{1}$ & 27 & 4 & 26 & 0.70522450087967 \\
8 & 0 & 23 & $0.84566812812502 \times 10^{-2}$ & 28 & 5 & 1 & 0.10770512626332 \\
9 & 1 & 2 & $0.12654315477714 \times 10^{1}$ & 29 & 5 & 3 & 0.32913623258954 \\
10 & 1 & 6 & $0.11524407806681 \times 10^{1}$ & 30 & 5 & 26 & 0.50871062041158 \\
11 & 1 & 15 & 0.88521043984318 & 31 & 6 & 0 & $0.22175400873096 \times 10^{-1}$ \\
12 & 1 & 17 & 0.64207765181607 & 32 & 6 & 2 & $0.94260751665092 \times 10^{-1}$ \\
13 & 2 & 0 & 0.38493460186671 & 33 & 6 & 26 & 0.16436278447961 \\
14 & 2 & 2 & 0.85214708824206 & 34 & 7 & 2 & $0.13503372241348 \times 10^{-1}$ \\
15 & 2 & 6 & $0.48972281541877 \times 10^{1}$ & 35 & 8 & 26 & $0.14834345352472 \times 10^{-1}$ \\
16 & 2 & 7 & $0.30502617256965 \times 10^{1}$ & 36 & 9 & 2 & $0.57922953628084 \times 10^{-3}$ \\
17 & 2 & 22 & $0.39420536879154 \times 10^{-1}$ & 37 & 9 & 26 & $0.32308904703711 \times 10^{-2}$ \\
18 & 2 & 26 & 0.12558408424308 & 38 & 10 & 0 & $0.80964802996215 \times 10^{-4}$ \\
19 & 3 & 0 & 0.27999329698710 & 39 & 10 & 1 & $0.16557679795037 \times 10^{-3}$ \\
20 & 3 & 2 & $0.13899799569460 \times 10^{1}$ & 40 & 11 & 26 & $0.44923899061815 \times 10^{-4}$ \\
\hline
\end{tabular}

Table A.2: Relations to obtain fluid properties Helmholtz Free Energy (IAPWS, 2007)

Pressure

$$
p=\rho^{2}(\partial f / \partial \rho)_{T} \quad \frac{p(\delta, \tau)}{\rho R T}=\delta \phi_{\delta}
$$

Specific Enthalpy

$$
h=f-T(\partial f / \partial T)_{\rho}+\rho(\partial f / \partial \rho)_{T} \quad \frac{h(\delta, \tau)}{R T}=\tau \phi_{\tau}+\delta \phi_{\delta}
$$

Specific Isobaric Heat Capacity

$$
c_{p}=(\partial h / \partial T)_{p}
$$$$
\frac{c_{p}(\delta, \tau)}{R}=-\tau^{2} \phi_{\tau \tau}+\frac{\left(\delta \phi_{\delta}-\delta \tau \phi_{\delta \tau}\right)^{2}}{2 \delta \phi_{\phi}+\delta^{2} \phi_{\delta \delta}}
$$

Specific Entropy

$$
s=-(\partial f / \partial T)_{\rho} \quad \frac{s(\delta, \tau)}{\rho R}=\tau \phi_{\tau}+\phi
$$


in Equation A.3. Here again, $\overline{\lambda_{0}}$ represents the conductivity at the the ideal gas limit, $\lambda_{1}$ represents the from the finite density, and $\lambda_{2}$ represents the enhanced conductivity due to the behaviour near the critical point. Details of the correlation can be obtained in Revised Release on the IAPWS Formulation 1985 for the Thermal Conductivity of Ordinary Water Substance (IAPWS 1998b).

$$
\bar{\lambda}=\overline{\lambda_{0}}(T) \times \lambda_{1}(T, \rho) \times \lambda_{2}(T, \rho)
$$

\section{A.3 Comparison of Thermodynamic Properties}

Thermodynamic and transport properties for the IAPWS-95 scientific formulation are obtained from the NIST database. A look-up table for the fluid properties within a pressure range of 20 and $30 \mathrm{MPa}$ and temperature range of $635 \mathrm{~K}$ to $750 \mathrm{~K}$, which are typical temperature and pressure ranges proposed for supercritical water reactors and correspond to the conditions of the current study, is compiled with temperature and pressure increments of $0.2 \mathrm{~K}$ and $0.1 \mathrm{MPa}$ respectively. The selection of these temperature and pressure increment sizes is discussed in Section A.4. An Excel Visual Basic program that calculates the properties of water following the IAPWS-97 industrial formulations are provided by Spang (2002). The contents of the program code are manually verified to be consistent with the IAPWS-97 standard. The IAPWS-97 data was projected onto an identical grid as the IAPWS-95 data. Data from the two formulations were plotted against each other to verify the agreement. The density, dynamic viscosity, thermal conductivity, and isobaric heat capacity $\left(c_{p}\right)$ are analyzed because the Navier-Stokes equations are dependant on these properties. Contour plots of these properties in terms of enthalpy and pressure are provided alongside traditional plots in terms of temperature and pressure because ANSYS CFX adopts the enthalpy formulation of the energy equation. These plots are shown in Figures A.2 


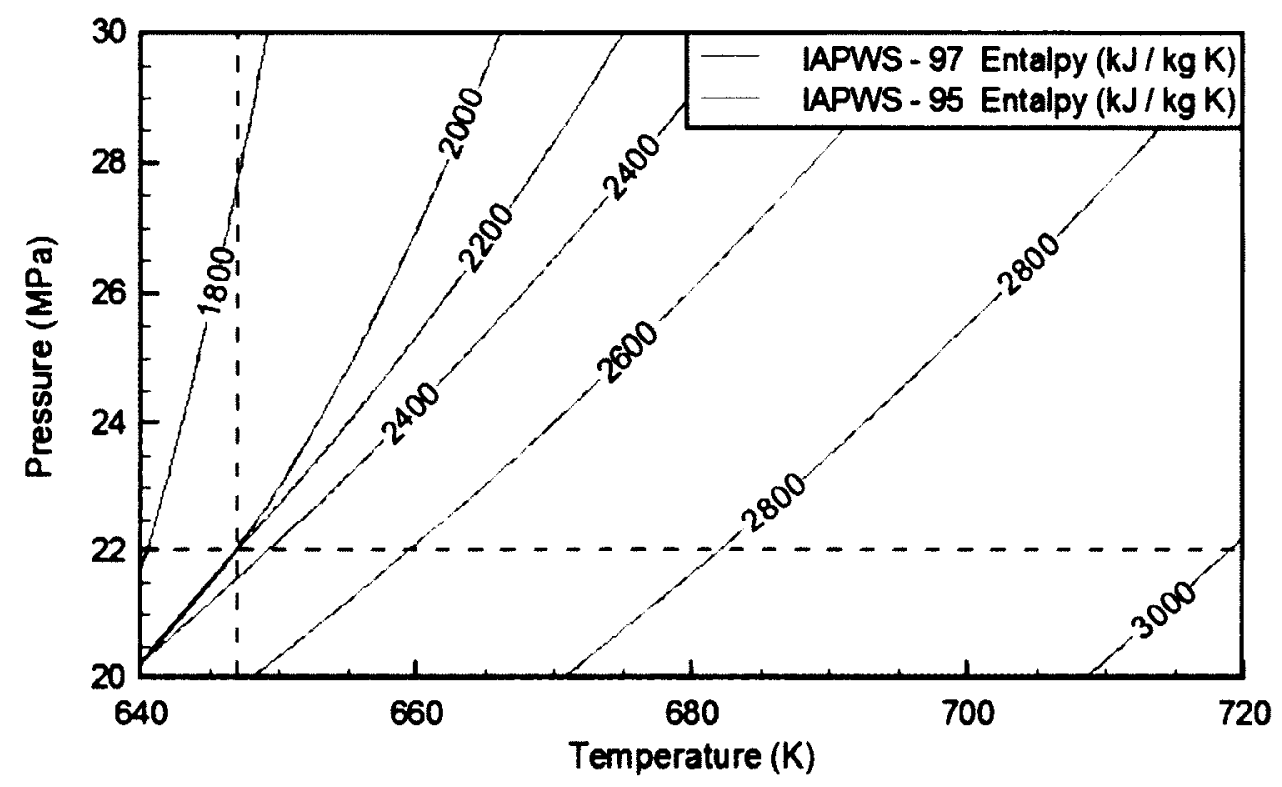

Figure A.2: Comparison of enthalpy vs pressure and temperature using the IAPWS-95 and IAPWS-97 formulations

to A.10. For each plot, the region surrounding the vapour dome is blanked. Dashed green lines indicate the critical pressure and critical temperature or enthalpy (the critical enthalpy is the enthalpy at the critical pressure and temperature).

Figures A.3 and A.4 compares supercritical water densities predicted by the IAPWS-95 and IAPWS-97. Except for the minor discrepancy slightly above the vapour dome, the densities produced by the IAPWS-95 and IAPWS-97 formulations are in good agreement, confirming that the industrial formulation is adequate for this property.

Specific isobaric heat capacity $\left(c_{p}\right)$ shows the most significant gradients near the critical point. Far from the critical point, $c_{p}$ remains below $10 \mathrm{~kJ} / \mathrm{kg} \mathrm{K}$. However, approaching the critical point, $c_{p}$ rises rapidly until it reaches a singularity at the critical point hence it has the steepest gradients amongst all thermodynamic properties. Although, there is reasonable agreement between IAPWS-95 data and IAPWS-97 data, 


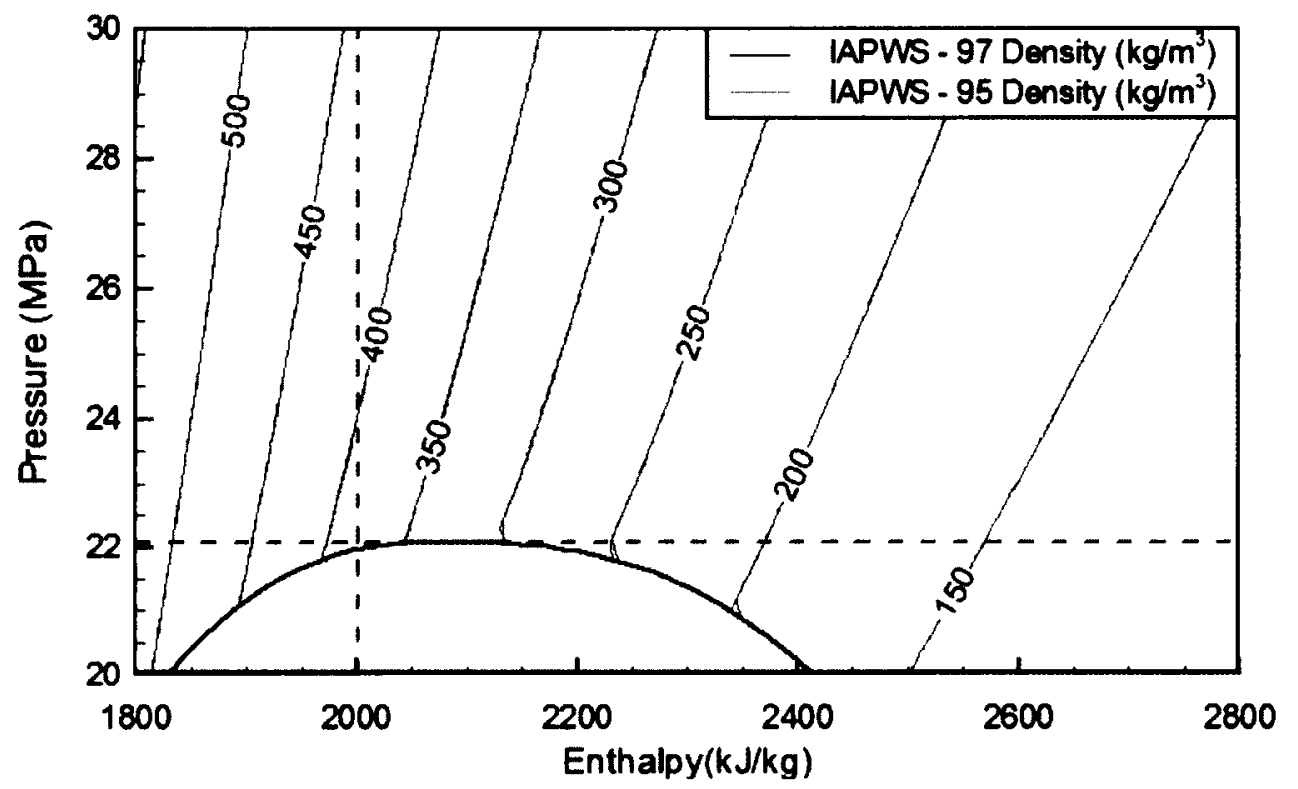

Figure A.3: Comparison of density vs pressure and enthalpy for NIST using the IAPWS-95 and IAPWS-97 formulations

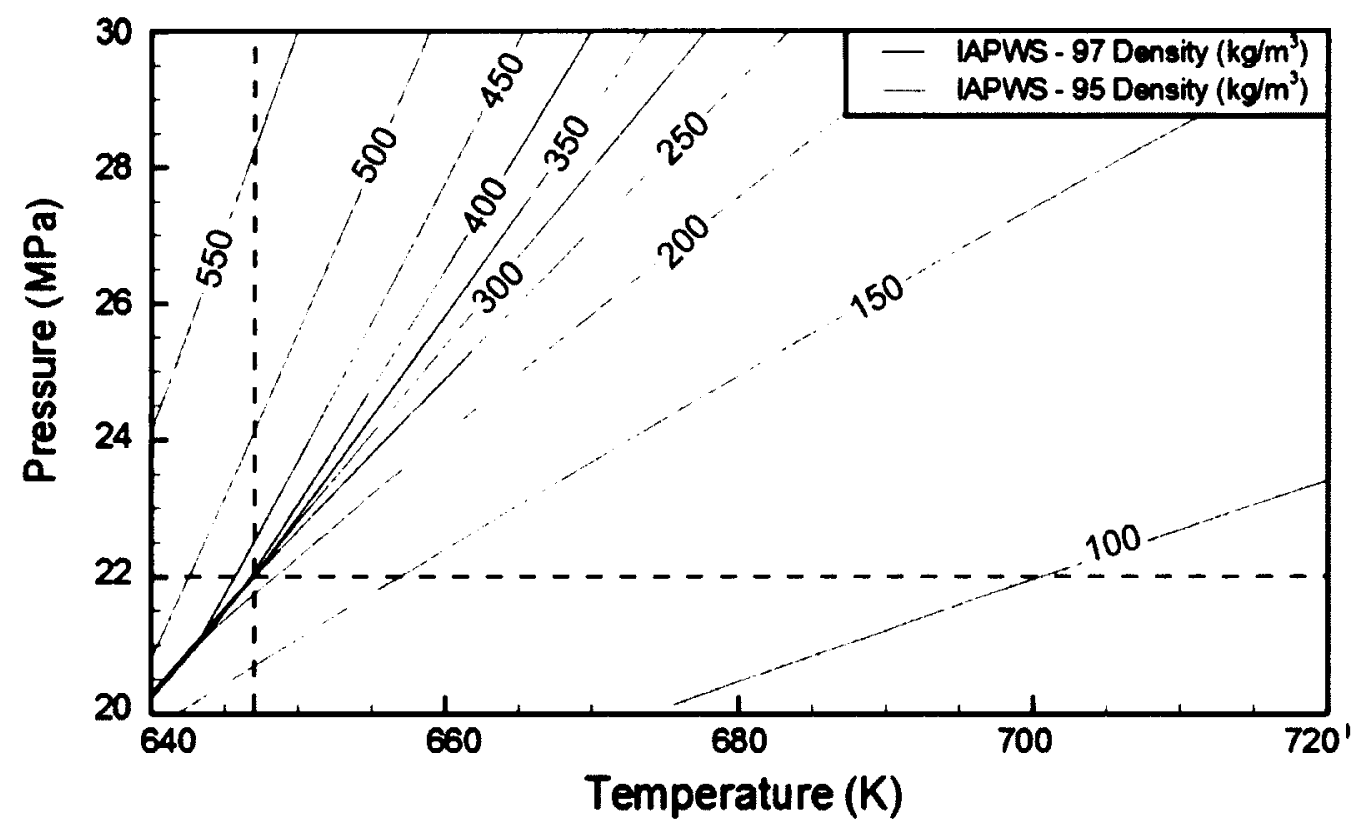

Figure A.4: Comparison of density vs pressure and temperature using the IAPWS-95 and IAPWS-97 formulations 


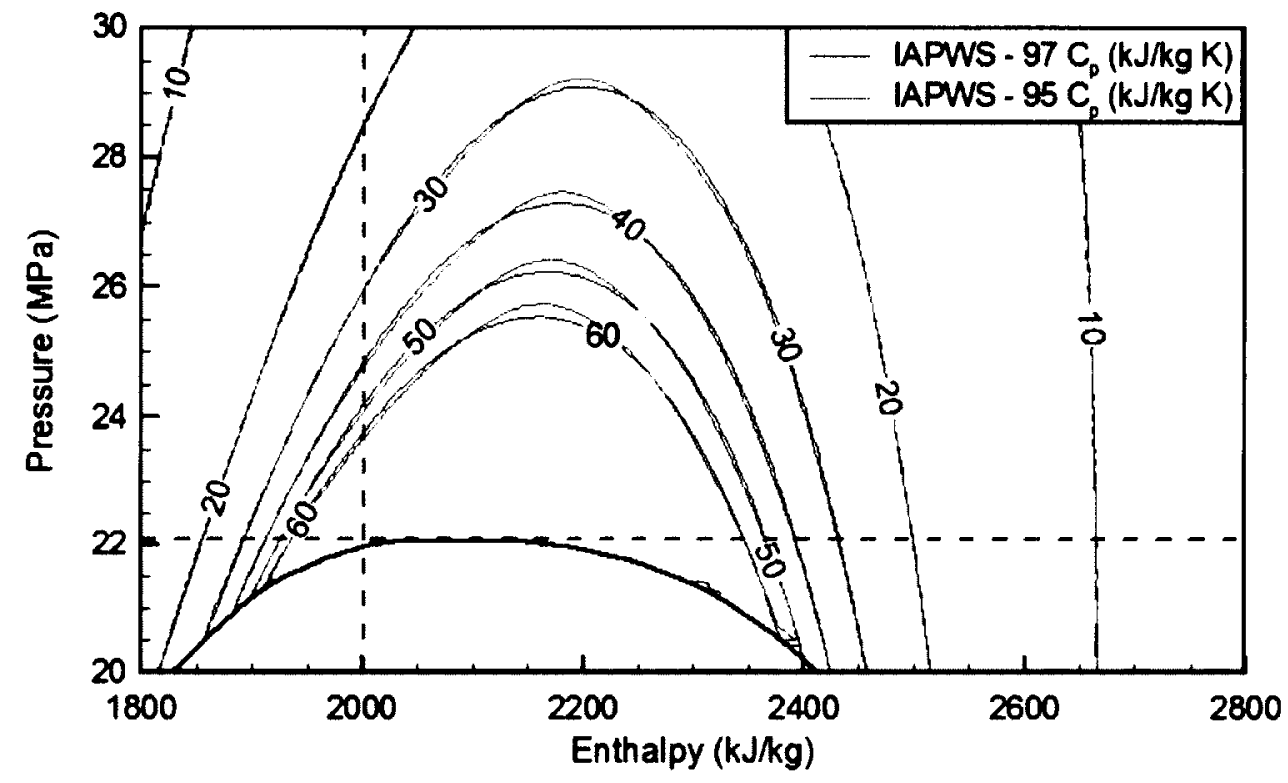

Figure A.5: Comparison of specific isobaric heat capacity vs pressure and enthalpy using the IAPWS-95 and IAPWS-97 formulations

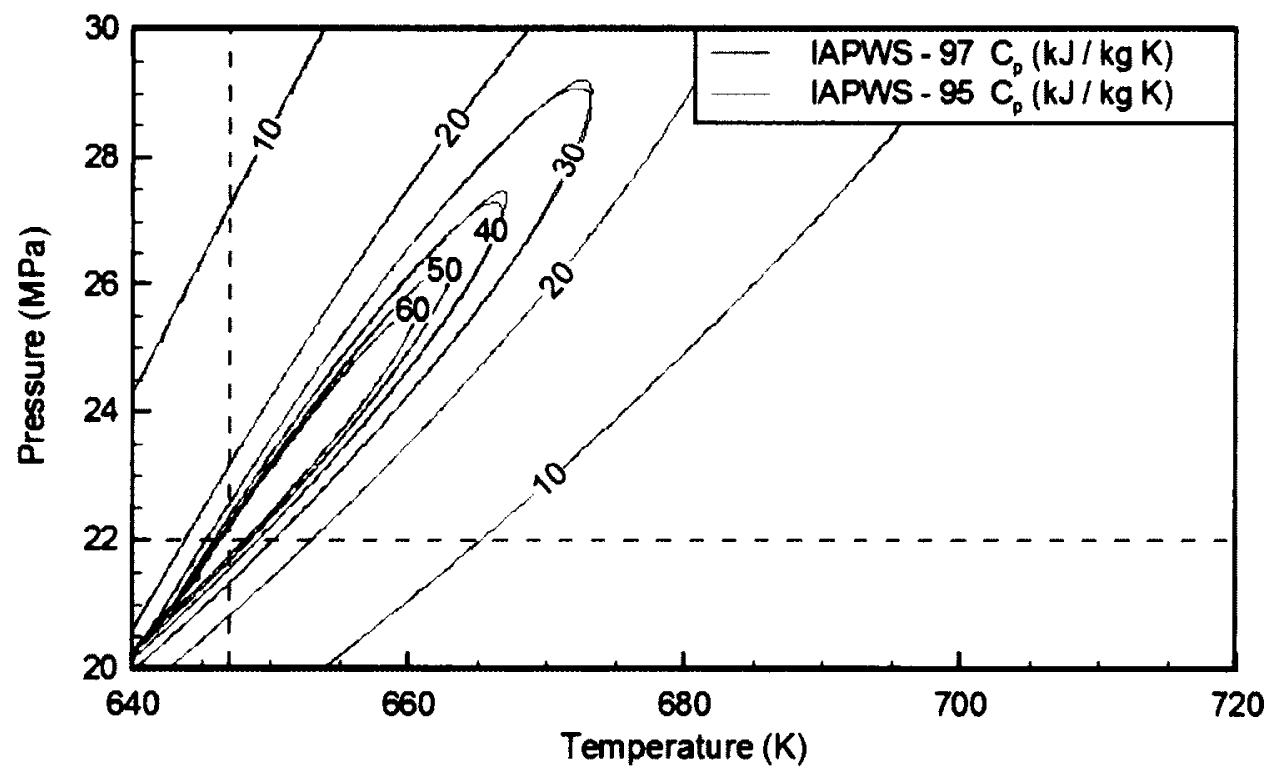

Figure A.6: Comparison of specific isobaric heat capacity vs pressure and temperature using the IAPWS-95 and IAPWS-97 formulations 
below $24 \mathrm{MPa}$, the discrepancy between the two formulations becomes significant $(\approx 2 \%$ relative difference ratio). The humps on the contours of the specific isobaric heat capacity, which correspond to the location of maximum $c_{p}$ for an isobar, are the locations of the thermodynamic pseudo-critical point. The discrepancy between the IAPWS-95 and IAPWS-97 formulation are the greatest at this point.

Figures A.7 and A.8 compares the dynamic viscosity using the IAPWS-95 and IAPWS-97 formulations. There is generally a good agreement between the two formulations with evident discrepancies near the vapour dome where the industrial formulation under predicts the viscosity. By approximately $23 \mathrm{MPa}$, the discrepancies between the two formulations are minimal $(<1 \%)$ suggesting that either formulation can be used to provide accurate predictions of dynamic viscosity.

Figure A.9 and A.10 compares the thermal conductivity between IAPWS-95 and IAPWS-97. As opposed to the previous properties, disparity between the thermal conductivity predictions is not solely localized near the pseudo-critical point and the vapour dome but can be seen throughout the property space. IAPWS-97 generally under predicts thermal conductivity with few exceptions. However it is clear from these plots that the total magnitude of the deviation between the two formulations are low $(<1 \%)$, especially when compared with the deviation of the correlations with experimental data Wagner and Prub (2001), discussed in section A.4.

In general, there appears to be a good agreement between the two sources suggesting that the choice of the database will not significantly affect the results. Therefore, the IAPWS-97 library which is implemented in ANSYS CFX appears adequate to define the properties of supercritical water for direct numerical simulation. 


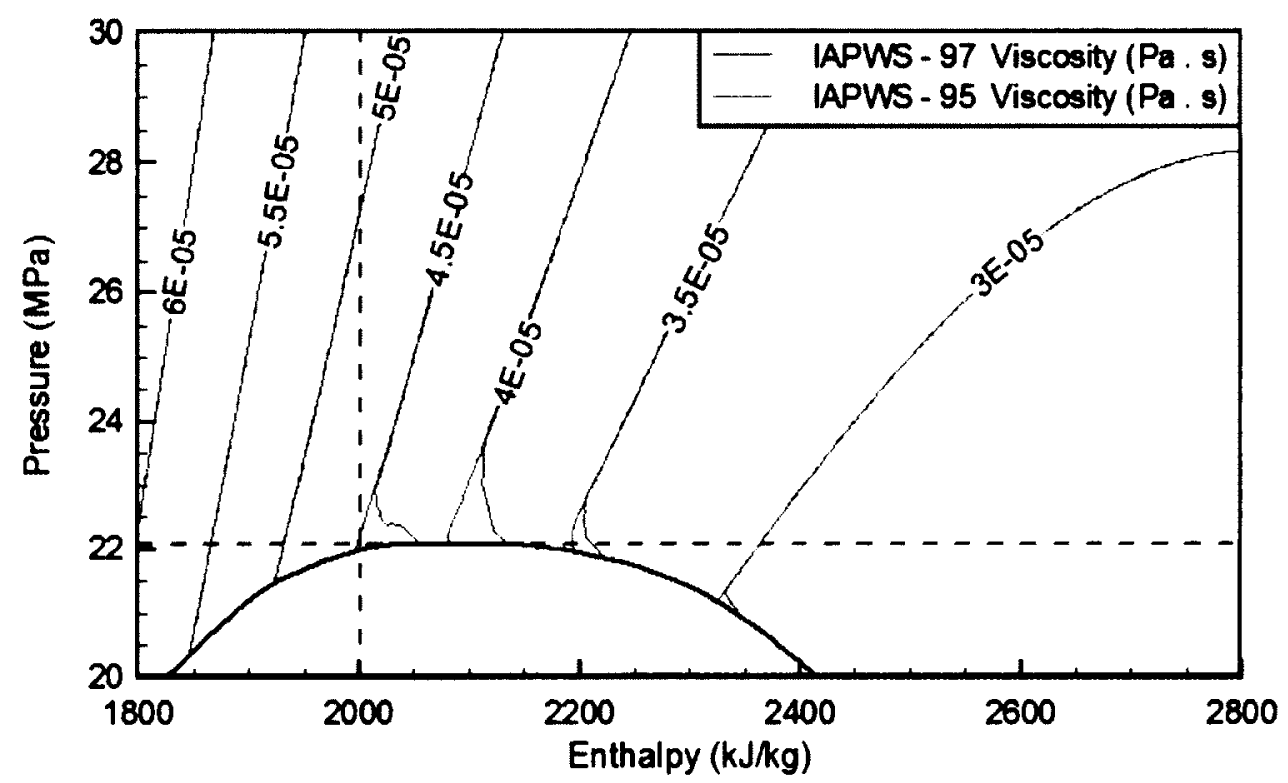

Figure A.7: Comparison of viscosity vs pressure and enthalpy using the IAPWS-95 and IAPWS-97 formulations

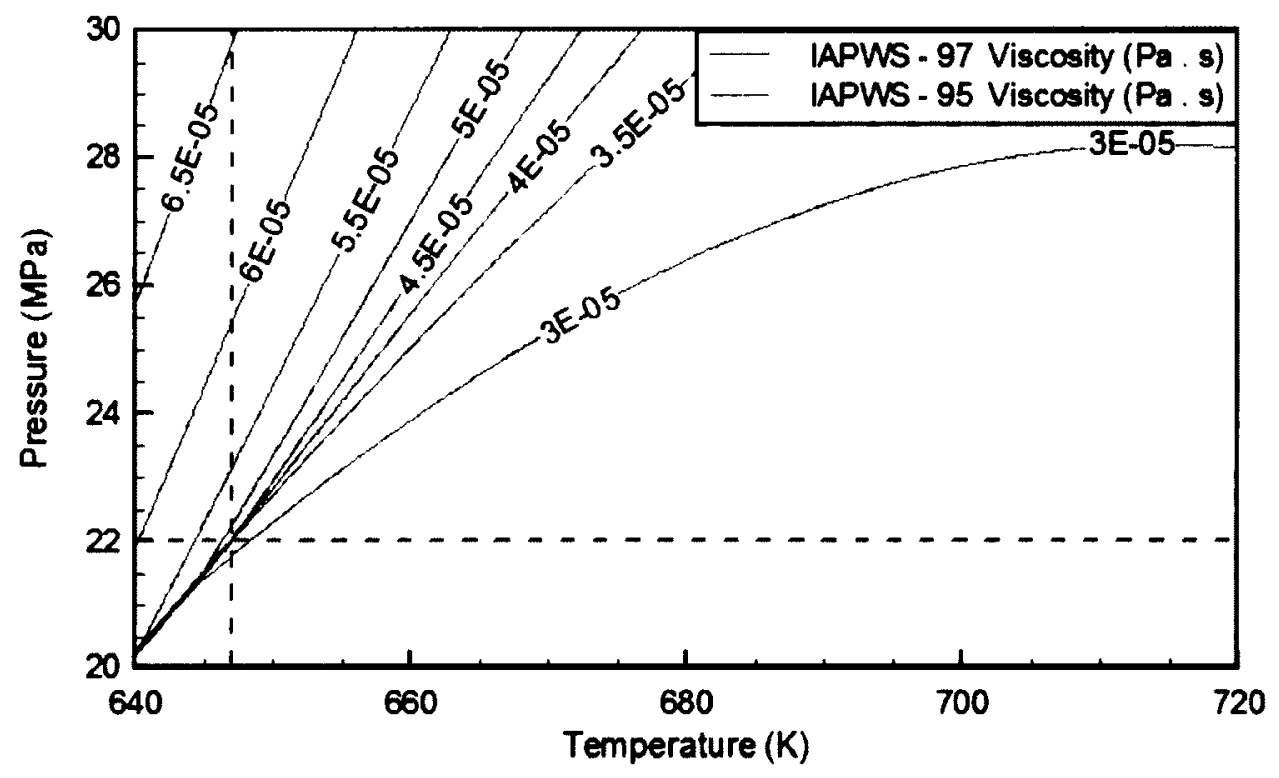

Figure A.8: Comparison of viscosity vs pressure and temperature using the IAPWS-95 and IAPWS-97 formulations 


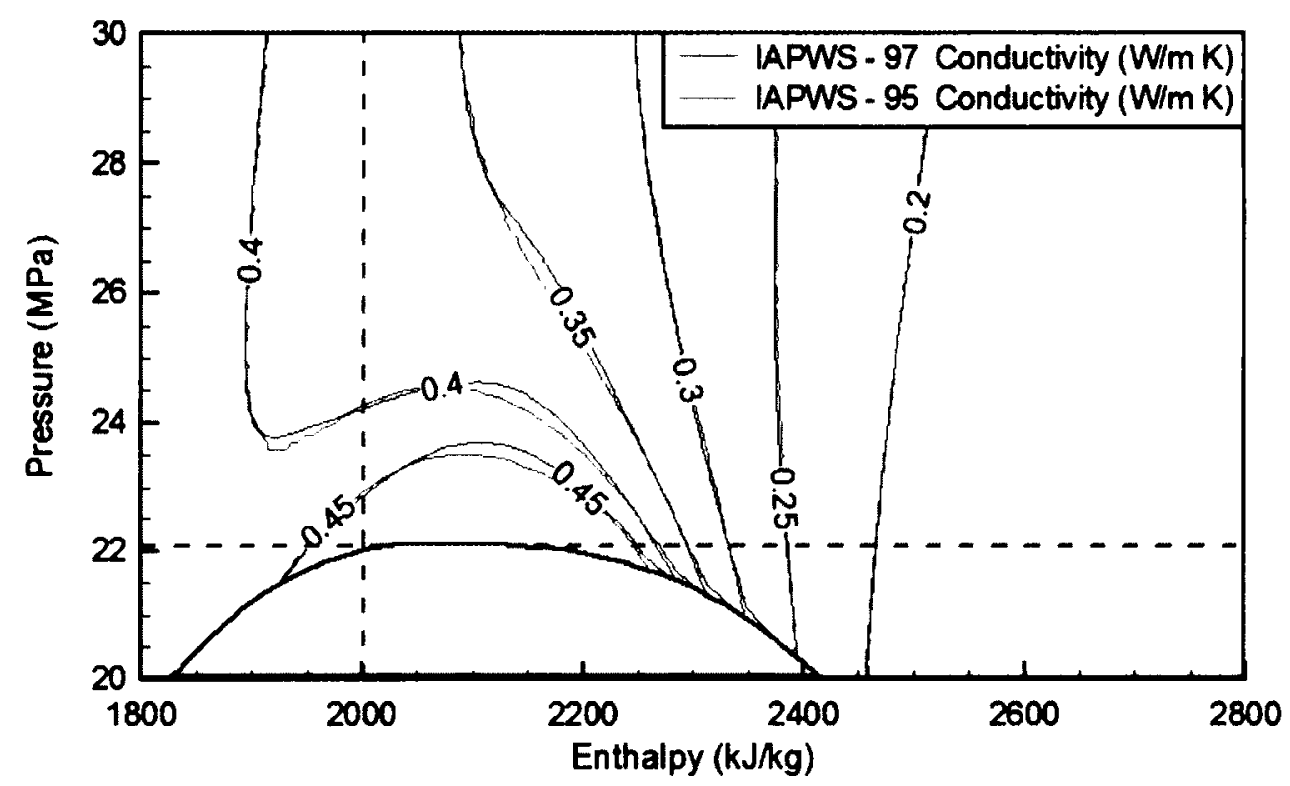

Figure A.9: Comparison of thermal conductivity contours vs pressure and enthalpy using the IAPWS-95 and IAPWS-97 formulations

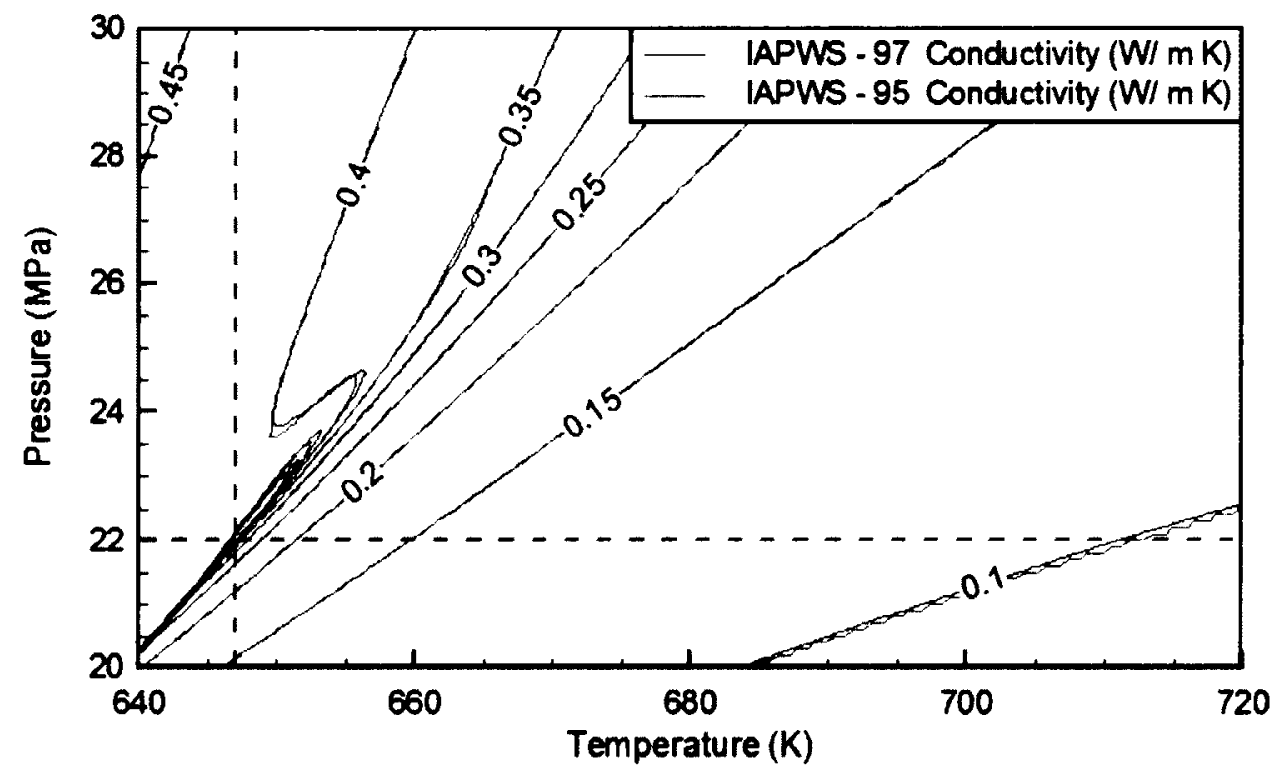

Figure A.10: Comparison of thermal conductivity contours vs pressure and temperature using the IAPWS-95 and IAPWS-97 formulations 


\section{A.4 Sizing of the Thermodynamic Grid}

Of the properties studied, $c_{p}$ has the largest gradients and thus the precision of the thermodynamic properties obtained from the look-up table is limited by how well $c_{p}$ is resolved. Owing to the appearance of the isobaric specific heat in the Prandl number $\left(P r=c_{p} \mu / k\right)$ in the enthalpy formulation of the energy equation, high specific heats contribute to the energy equation by reducing the impact of heat flux in the form of diffusion and increasing the relative importance of convection. More importantly, $c_{p}$ is the gradient of enthalpy with temperature and thus resolving this parameter is tantamount to sizing the grid with respect to the curvature of enthalpy with respect to temperature. In many schemes, the curvature is equally as important as the gradient for interpolation. Both the enthalpy and isobaric heat capacity are the relevant quantities in the energy equation. At the critical point, the gradient of enthalpy and the gradient of specific isobaric heat capacity with respect to temperature are undefined. However, slightly away from the critical point, the relative change of enthalpy with respect to temperature is milder than the relative change of specific isobaric heat capacity with temperature and the relative change of enthalpy with respect to pressure is milder still. Thus sizing the thermodynamic grid based on enthalpy is more lenient therefore a more conservative approach will be taken by using the isobaric heat capacity for grid resolution studies.

From a numerical standpoint it is important not to have an over-refined grid for the thermodynamic properties as it will result in increased time required for interpolation with minor improvements in accuracy, however, too course a grid will result in faster interpolation but decreased accuracy. Thus a criterion must be developed to ensure that a suitable grid is selected. The experimental studies published from Wagner and Prub (2002) suggest that a scatter of approximately $8 \%$ in $c_{p}$ near the pseudo critical temperature at $24 \mathrm{MPa}$, the design point of interest, as seen in Figure A.11. Any 


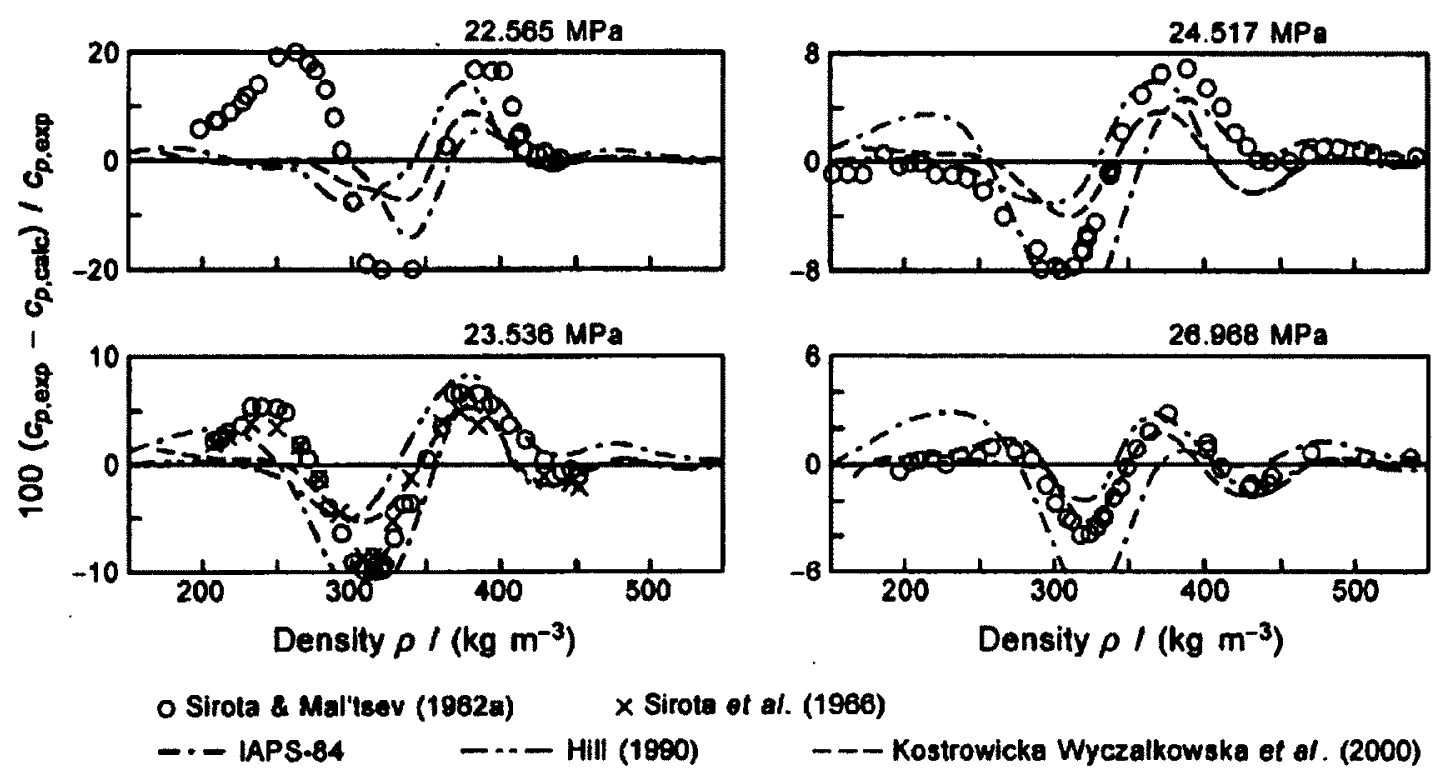

Figure A.11: Uncertainty of IAPWS-95 formulation compared to experimental data (Wagner and Prub, 2002)

grid that predict $c_{p}$ for a given temperature and pressure that is $8 \%$ different from the correlations is not consistent with experiment. Therefore, the criteria that an $8 \%$ change in $c_{p}$ must be captured is required to ensure precision better than experiments and thus this criteria constitutes a necessary condition. In practise, the interpolation schemes used by CFX and programs using the thermodynamic grid will ensure an approximation far better than $8 \%$ of the true data since a continuously varying $c_{p}$ will be obtained between points on the thermodynamic grid.

For a given thermodynamic process where there is a small change in temperature and pressure, the associated change in $c_{p}$ is:

$$
\Delta c_{p}=\frac{\partial c_{p}}{\partial T} \Delta T+\frac{\partial c_{p}}{\partial P} \Delta P
$$


For a given isobaric process, the above relation can be expressed as :

$$
\Delta c_{p}=\frac{\partial c_{p}}{\partial T} \Delta T
$$

For a given isothermal process, the above relation can be expressed as:

$$
\Delta c_{p}=\frac{\partial c_{p}}{\partial P} \Delta P
$$

The discrepancy of $\pm 8 \%$ in $c_{p}$ can be expressed as the mathematical criteria that $\Delta c_{p} / c_{p}=0.08$. Taking this and rearranging equation A.5 and A.6, it is possible to find adequate temperature and pressure spacing results in the following equations:

$$
\begin{gathered}
\Delta T=\frac{0.08}{\frac{1}{c_{p}} \frac{\partial c_{p}}{\partial T}} \\
\Delta P=\frac{0.08}{\frac{1}{c_{p}} \frac{\partial c_{p}}{\partial P}}
\end{gathered}
$$

Figure A.12 shows the variation of the $c_{p}$ and the non-dimensional $c_{p}$ gradient with respect to temperature taken at $24 \mathrm{MPa}$. The design point is set to $24 \mathrm{MPa}$ because exactly at the critical point the gradients in $c_{p}$ are too large to practically resolve any thermodynamic grid. Figure A.12 shows that the maximum non-dimensional gradient occur at $0.7 \mathrm{~K}$ from the pseudocritical point with a magnitude of 0.6 . Figure A.13 shows the variation of $c_{p}$ and the non-dimensional $c_{p}$ gradient with respect to pressure taken at the critical temperature $(647 \mathrm{~K})$. The gradient in $c_{p}$ is undefined at the critical pressure of $22.1 \mathrm{MPa}$, however, reaches a reasonable value of 0.3 at the design pressure of $24 M P a$. This data in conjunction with Eqn.A.7 and A.8 may be used to predict temperature and pressure increments of $0.1 \mathrm{~K}$ and $0.2 \mathrm{MPa}$ respectively. The data obtained through this analysis is recorded on Table A.3. 


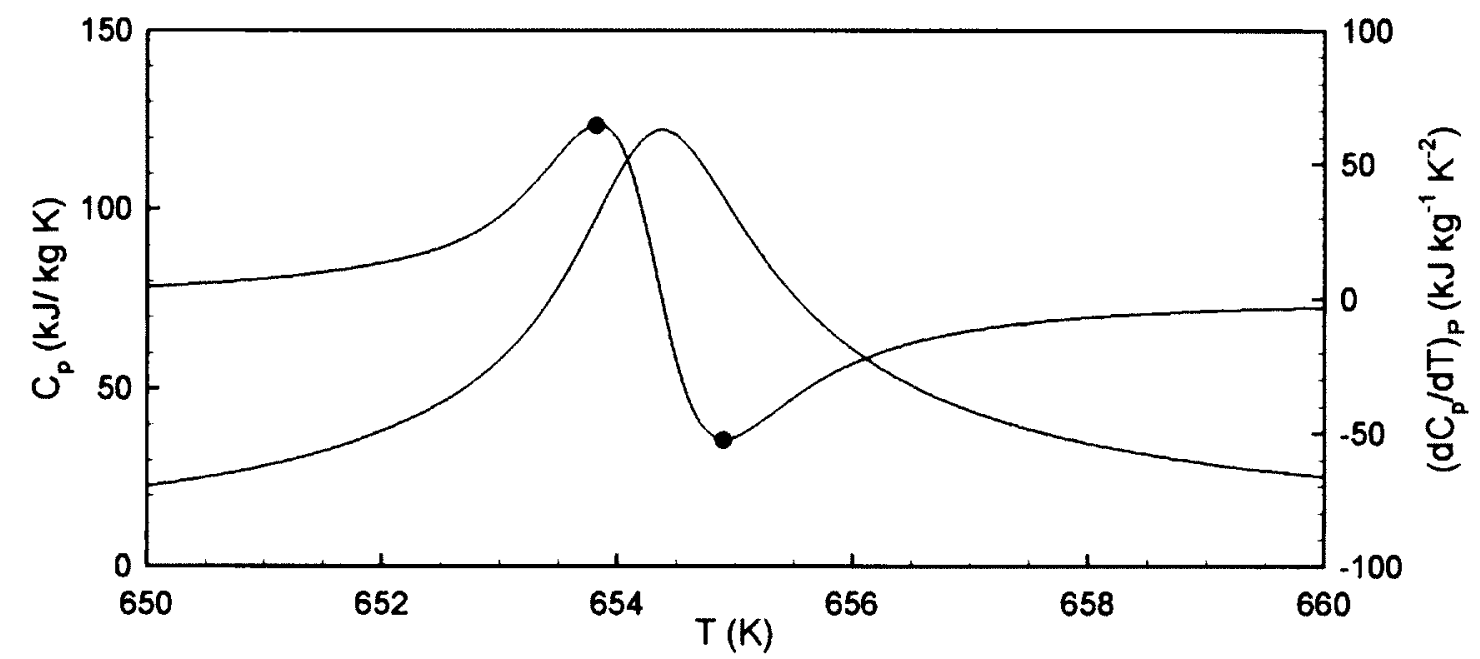

Figure A.12: Variation of $c_{p}$ against temperature at $P=24 \mathrm{MPa}$. Red $c_{p}$. Green: Non dimensional $c_{p}$ variation $\frac{\partial \mathbf{C}_{p}}{\partial T}$

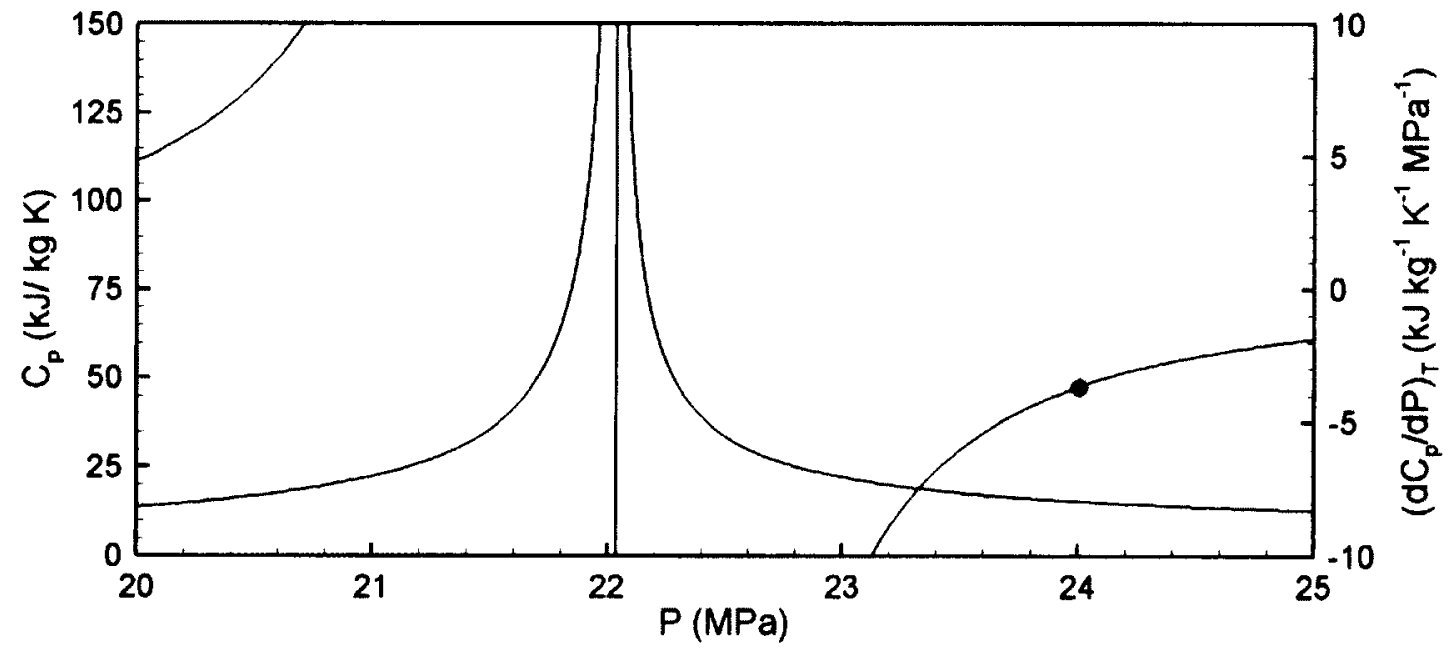

Figure A.13: Variation of $c_{p}$ against pressure at $T=647 \mathrm{~K}$. Red $c_{p}$. Green Non dimensional $c_{p}$ variation $\frac{\partial \mathbf{C}_{p}}{\partial P}$ 
Table A.3: Recommended dimensions of thermodynamic grid

\begin{tabular}{ll}
\hline Parameter & Value \\
\hline Minimum Temperature & $600 \mathrm{~K}$ \\
Maximum Temperature & $720 \mathrm{~K}$ \\
Minimum Pressure & $20 \mathrm{MPa}$ \\
Maximum Pressure & $30 \mathrm{MPa}$ \\
Temperature Increment & $0.2 \mathrm{~K}$ \\
Pressure Increment & $0.1 \mathrm{MPa}$ \\
\hline
\end{tabular}

\section{A.5 Thermodynamic Grid Convergence Analysis}

The ssensitivity to the pressure and temperature increments used in the fluid property look-up table was assessed through a series of zero-pressure-gradient boundary layer simulations in which the look-up table increments were incrementally refined until insensitivity to the increment size was obtained. A computational domain of extents of a $300 \mathrm{~mm} \times 60 \mathrm{~mm} \times 40 \mathrm{~mm}$ in the streamwise, wall-normal and spanwise directions respectively was created. Nodes were clustered around the boundary layer and a noslip isothermal lower surface was specified. A top hat profile of $U=0.1 \mathrm{~m} / \mathrm{s}$ with a spatially-uniform temperature of $670 \mathrm{~K}$ is specified at the inlet. The no-slip wall is maintained at the critical temperature of $647 \mathrm{~K}$ so that the temperature difference between the wall and the bulk fluid provides the conditions for heat transfer. The temperature range between $647 \mathrm{~K}$ and $670 \mathrm{~K}$ is chosen because temperature interval contains the most significant property gradients.

IAPWS-95 water tables obtained from the NIST thermophysical properties website (NIST, 2011) were formatted into real gas property (RGP) data files that are an input to CFX to specify fluid property. These two property look up tables covered a temperature range of $600 \mathrm{~K}$ to $720 \mathrm{~K}$ and a pressure range of $20 \mathrm{MPa}$ to $30 \mathrm{MPa}$. The resolution of the coarsest grid consists of pressure increments of $1 \mathrm{MPa}$ and temperature increments of $2 \mathrm{~K}$. The moderate sized property tables consisted 
of temperature increments of $0.2 \mathrm{~K}$ and pressure increments of $0.1 \mathrm{MPa}$. The finest grids were provided through the use CFX's built in IAPWS-97 databases and its temperature resolution is $0.1 \mathrm{~K}$ and pressure resolution is $0.01 \mathrm{MPa}$.

The numerical setup consisting of the course, moderate and fine simulations were solved using Ansys CFX using the steady state assumption. The property variation in the wall normal direction near the trailing edge of the domain is provided in Figure A.14. The error values for the coarse and moderate sized grids were the percent difference when compared with the finest grids using the IAPWS-97 tables provided by CFX. This is specified below where $\phi$ represents a property such as density.

$$
\% \text { Error }=\frac{\phi-\phi_{I A P W S 97, C F X}}{\phi_{I A P W S 97, C F X}} \times 100 \%
$$

Figure A.14 shows the property variations using the three thermodynamic grids for density, viscosity, temperature, and streamwise velocity. The coarsest thermodynamic grids show a significantly impaired ability to predict the heat transfer over the viscous regions of interest. This discrepancy is most pronounced at $0.4 \mathrm{~mm}$ where the fluid is at the pseudocritical temperature for the $24 \mathrm{MPa}$ isobar. The discrepancy in density and viscosity approach $7 \%$ for density and dynamic viscosity. However, the moderately resolved thermodynamic grids show very low discrepancies to a maximum of approximately $1 \%$ for density. The curves obtained using the moderately resolved grids are almost indistinguishable from the fine case suggesting that the moderate thermodynamic grid is adequate for capturing the property variation through heat transfer in a boundary layer. As a result, these studies confirm the suitability of the thermodynamic table specifications provided in Table A.3. 


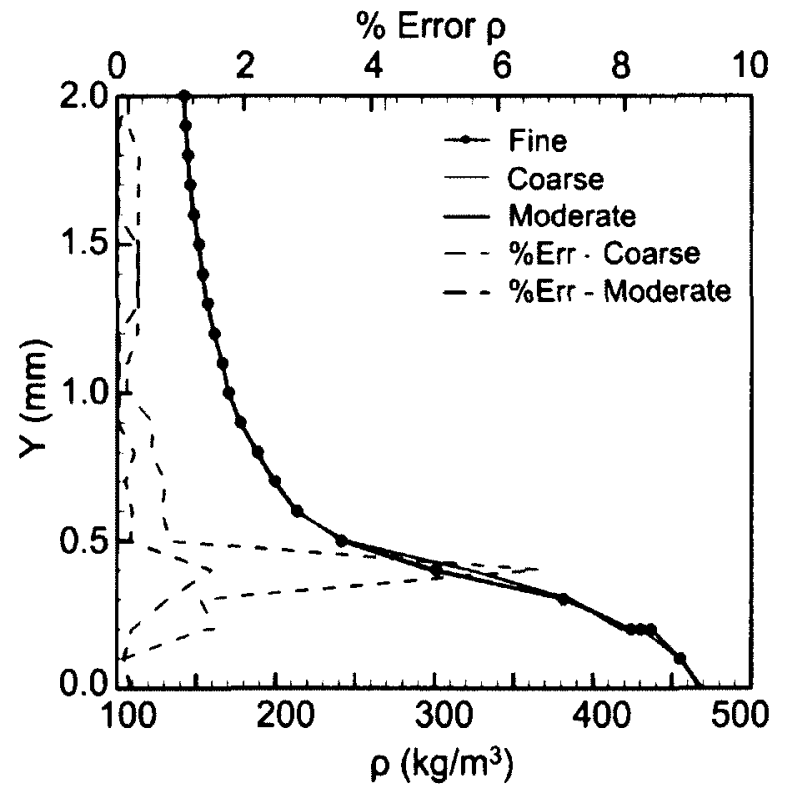

(a)

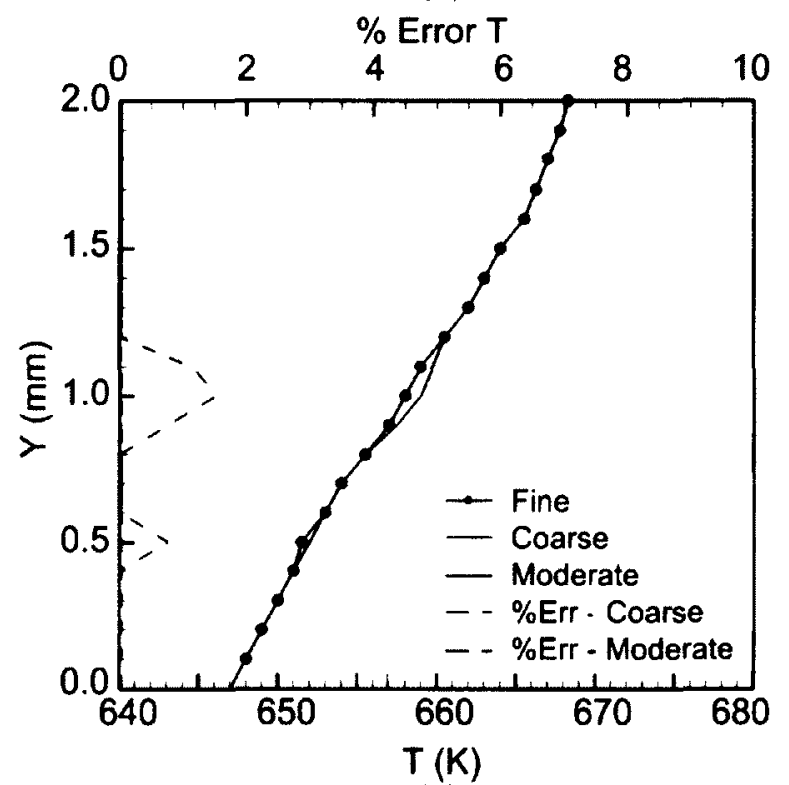

(c)

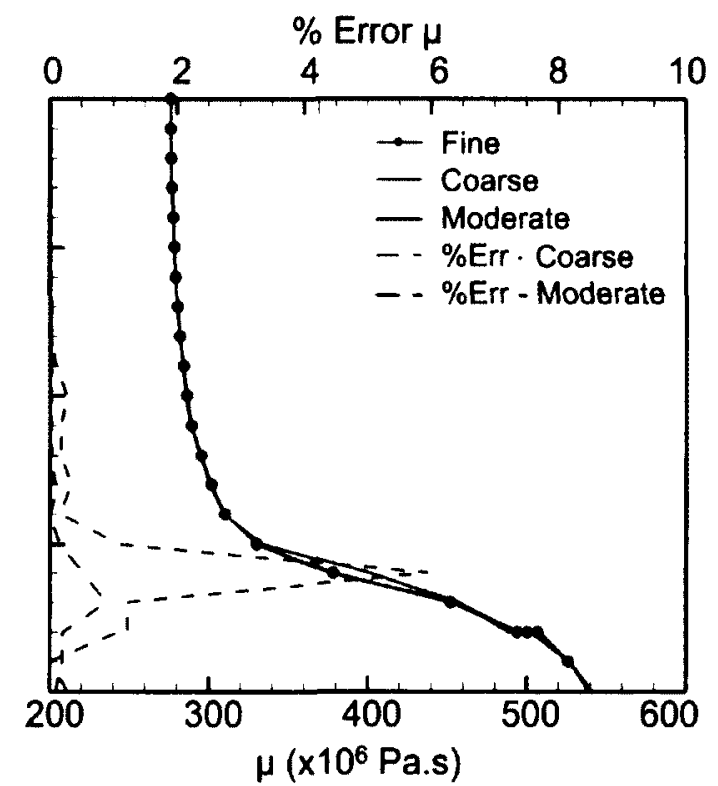

(b)

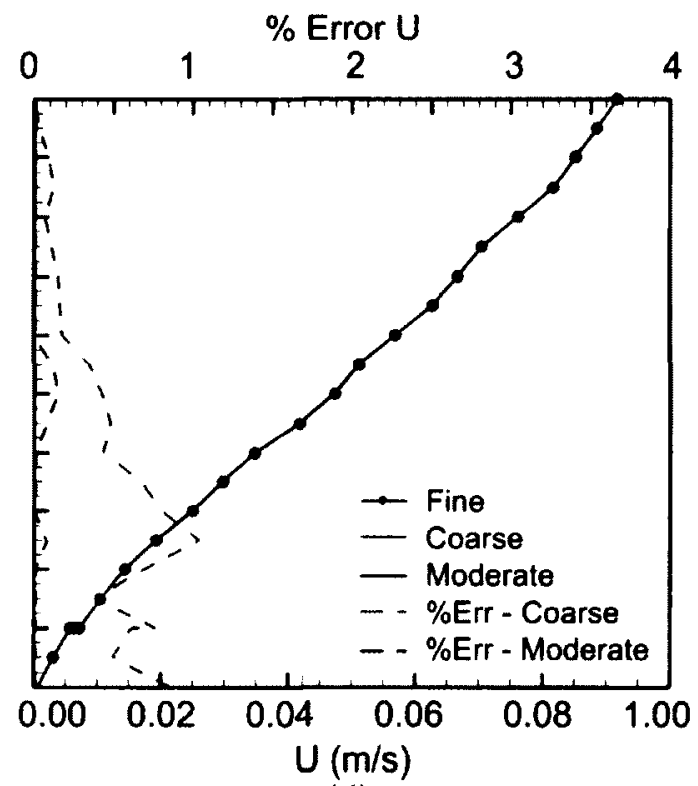

(d)

Figure A.14: Wall-normal variation in (a) density, (b) dynamic viscosity, (c) temperature, and (d) velocity magnitude at $x / L=0.9$ and $z / L=0.5$ for simulations with fine, medium, and coarse fluid-property increment sizes. Dashed lines show relative error for each distribution. 


\section{Appendix B}

\section{Data Analysis Procedures}

\section{B.1 Overview}

This appendix describes: the methodology used to analyze the time-varying field data obtained from the solution of the Navier-Stokes equations; linear stability analysis used to assess the stability of the shear-layer profiles; and the potential flow models that assist the interpretation of the computational data produced through the solution of the Navier-Stokes equations. Specifically, the chapter begins with a discussion on the similarity parameters used to assess the dynamic-similarity of the test cases in Section B.2 followed by a description of the statistical tools used to interpret the fluctuations in flow parameters in Section B.3. Sections B.4, B.5 and B.6 discuss the approaches used to calculate various integral, differential and spectral quantities pertaining to the flow field. Section B.7 provides a description of the linear stability theory used to find the eigenvalues and eigenmodes of the separated shear-layer profile. Sections 3.4.1 and 3.4.2 describe the steady and transient potential flow codes used to explain the trends in delayed transition and vorticity interaction in the shear-layer in close proximity to a wall as observed through the solution of the Navier-Stokes equations. Finally, Section B.8 discusses the grid convergence study for the shear layer simulations in Chapter 4. 


\section{B.2 Selection of Similarity Parameters}

It is believed that the time-evolution of the flow-field of a shear layer in close proximity to a wall including the frequency of the most- amplified instability $\left(f_{M A}\right)$ and the location of transition-onset $\left(x_{s-t r}\right)$, are dependant on the high-momentum-stream velocity $\left(U_{1}\right)$, the low-momentum-stream velocity $\left(U_{2}\right)$, the dynamic-viscosity $(\mu)$, the density $(\rho)$, the momentum-thickness at the high momentum stream trailing-edge $\left(\theta_{s}\right)$, and the proximity of the free shear-layer from the wall $\left(y_{s}\right)$ (Ho and Huerre, 1984; Diwan and Ramesh, 2009):

$$
x_{s-t r}, f_{M A}=f\left(U_{1}, U_{2}, y_{s}, \theta_{s}, \rho, \mu\right)
$$

Application of Buckingham's Pi theorem to this functional expression yields the following equation involving non-dimensional parameters:

$$
R e_{x_{s-t r}}, S t_{\theta, M A}=f\left(R e_{\theta}, R, Y_{s}\right)
$$

Where $R e_{x_{s-t r}}=U_{1} x_{s-t r} / \nu$ is the Reynolds number of transition onset, $S t_{\theta, M A}=$ $f_{M A} \theta / U_{1}$ is the most amplified Strouhal number, $R e_{\theta}=U_{1} \theta / \nu$ is the Reynolds number at the high momentum stream trailing-edge, $R=\left(U_{1}-U_{2}\right) /\left(0.5\left(U_{1}+U_{2}\right)\right)$ is the velocity difference ratio, and $Y_{s}=y_{s} / \theta$ is the non-dimensional wall proximity.

Similarly the dynamic-similarity of the supercritical-water cases are additionally dependent on the mean dynamic-viscosity $\bar{\mu}=0.5\left(\mu_{1}+\mu_{2}\right)$, the mean-density $\bar{\rho}=0.5\left(\rho_{1}+\rho_{2}\right)$, the mean isobaric heat capacity $\overline{c_{p}}=0.5\left(c_{p, 1}+c_{p, 2}\right)$, the mean temperature $\bar{T}=0.5\left(T_{1}+T_{2}\right)$, the mean conductivity $\bar{k}=0.5\left(k_{1}+k_{2}\right)$, and the pseudocritical temperature for the isobar $\left(T_{p c}\right)$ (Azih, 2012). As discussed in Chapter 2 and Appendix A, the pseudocritical point is temperature of maximum isobaric heat 
capacity for a given isobar. This relationship is expressed in Equation B.3.

$$
x_{s-t r}, f_{M A}=f\left(U_{1}, U_{2}, \theta, \bar{\rho}, \bar{\mu}, \bar{k}, \overline{c_{p}}, \bar{T}, T_{p c}\right)
$$

Through the application of Buckingham's Pi theorem, the corresponding functional relationship involving nondimensional parameters can be derived as:

$$
R e_{x_{s-t r}}, S t_{\theta, M A}=f\left(R e_{\theta}, R, \operatorname{Pr}, \bar{T}^{*}\right)
$$

Where $\operatorname{Pr}=\overline{c_{p}} \bar{\mu} / \bar{k}$ is Prandtl number at the shear layer meanline and $\bar{T}^{*}=\bar{T} / T_{p c}$ is a nondimensional temperature at the shear layer meanline above the pseudo-critical temperature.

\section{B.3 Calculation of Flow Statistical Quantities}

This section describes the statistical procedures used to study the transient data. The time-averaged velocity-field is calculated using the statistical mean of the pressure and velocity-components $(\mathrm{U}, \mathrm{V}, \mathrm{W}, \mathrm{P})$ based on 1024 discrete samples:

$$
\bar{U}=\frac{\sum_{i=1}^{1024} U_{i}}{1024}
$$

A total of 1024 timesteps are used in the averaging process reflecting the total number of timesteps collected for analysis. With 1024 timesteps, approximately 100 cycles of vortex shedding were observed with approximately 10 timesteps between shedding cycles. This temporal resolution of the flow-field was deemed sufficient based on repeated trials to accurately capture the highest frequency events in the simulations while the total of 100 shedding cycles was sufficient to get good estimates of the mean and standard-error of vortex shedding. 
The time-averaged interval of vortex-shedding is determined based on approximately $n=100$ instances of vortex-shedding-cycles: B.7.

$$
\begin{gathered}
\overline{\mathscr{T}}=\frac{\sum_{i=1}^{100} \mathscr{T}_{i}}{100} \\
\bar{f}=\frac{1}{\overline{\mathscr{T}}}
\end{gathered}
$$

Root-mean-square (rms) averaging is used for the fluctuating component of flow/fluid parameters:

$$
u_{r m s}=\sqrt{\frac{\sum_{i=1}^{100}\left(U_{i}-\vec{U}\right)^{2}}{100}}
$$

The standard-deviation of the vortex-shedding period $\left(s_{\mathscr{T}}\right)$ is used to assess the spread in the vortex-shedding data:

$$
s_{. \mathscr{T}}=\sqrt{\frac{1}{99} \sum_{i=1}^{99}\left(\mathscr{T}_{i}-\overline{\mathscr{T}}\right)^{2}}
$$

The standard-deviation on the frequency $s_{f}$ is calculated by scaling the standarddeviation on the period by the magnitude of the mean-frequency, as shown in Equation B.10.

$$
s_{f}=\frac{\bar{f}}{\bar{T}} s_{\mathscr{T}}
$$

The precision on all calculated data consists of the systematic and random error. The random error consists of the error due to the spread on the data and can be described by the standard-error (SE), show in Equation B.11 (Tavoularis 2005).

$$
S E=\frac{s}{\sqrt{100}}
$$




\section{B.4 Calculation of Flow Differential Parameters}

Post-processing of all first-order derivatives is performed through central-differencing which is formally second-order accurate on a uniform grid. For example, the streamwise-velocity gradient $\partial U / \partial x$ is computed through (Anderson, 1995):

$$
\frac{\partial U}{\partial x}=\frac{U_{i+1}-U_{i-1}}{2 \Delta x}
$$

where $i$ represents the node under consideration, $U$ is a sample velocity component, and $x$ is a sample direction. The above expression is second-order accurate, thereby remaining consistent with order of accuracy of the solver.

For uneven grid spacing, Equation B.12 is replaced by:

$$
\frac{\partial U}{\partial x}=\frac{U_{i+1}\left(x_{i}-x_{i-1}\right)^{2}-U_{i}\left(x_{i}-x_{i-1}\right)^{2}+U_{i}\left(x_{i+1}-x_{i}\right)^{2}-U_{i-1}\left(x_{i+1}-x_{i}\right)^{2}}{\left(x_{i+1}-x_{i}\right)\left(x_{i}-x_{i-1}\right)\left(x_{i+1}-x_{i-1}\right)}
$$

Central differencing is also used for the second-order derivatives. As an example, $d^{2} u / d x^{2}$ is estimated on uniform and uniform grid-node distributions as per the following equations, respectively (Anderson, 1995):

$$
\begin{gathered}
\frac{\partial^{2} U}{\partial x^{2}}=\frac{U_{i+1}-2 U_{i}+U_{i-1}}{(\Delta x)^{2}} \\
\frac{\partial^{2} U}{\partial x^{2}}=\frac{U_{i+1}\left(x_{i}-x_{i-1}\right)-U_{i}\left(x_{i+1}-x_{i-1}\right)+U_{i-1}\left(x_{i+1}-x_{i}\right)}{0.5\left(x_{i+1}-x_{i-1}\right)\left(x_{i+1}-x_{i}\right)\left(x_{i}-x_{i-1}\right)}
\end{gathered}
$$

The streamwise, wall-normal and spanwise-vorticity components at any point in the flowfield are determined from the spatial gradients of velocity components as per the following equations:

$$
\omega_{x}=\frac{\partial w}{\partial y}-\frac{\partial v}{\partial z}
$$




$$
\begin{aligned}
& \omega_{y}=\frac{\partial u}{\partial z}-\frac{\partial w}{\partial x} \\
& \omega_{z}=\frac{\partial v}{\partial x}-\frac{\partial u}{\partial y}
\end{aligned}
$$

The vorticity-magnitude is defined as given in Equation B.19.

$$
|\Omega|=\sqrt{\omega_{x}^{2}+\omega_{y}^{2}+\omega_{z}^{2}}
$$

\section{B.5 Calculation of Flow Integral Parameters}

The procedure adopted for the numerical integration of all integral quantities is the trapezoidal-method, shown in Equation B.20, due to its versatility for uneven grids (Recktenwald, 2000):

$$
\int_{a}^{b} U(x) d x \approx \frac{1}{2} \sum_{i=1}^{N}\left(x_{i}-x_{i-1}\right)\left(U\left(x_{i}\right)+U\left(x_{i-1}\right)\right)
$$

The boundary-layer thickness $(\delta)$ is defined as the location where the local velocity magnitude is $99 \%$ of the local freestream velocity. Similarly, the thermal boundarylayer-thickness $\left(\delta_{T}\right)$ is defined as the first wall-normal location where the temperature reaches $99 \%$ of the local freestream temperature.

The momentum thickness is a length scale that represents the momentum deficit in a shear-layer due to viscous effects. The momentum thickness for a boundary layer is calculated using (White, 1992):

$$
\theta=\int_{0}^{\delta} \frac{\rho(y) U(y)}{\rho_{f} U_{f}}\left(1-\frac{U(y)}{U_{f}}\right) d y
$$


For a free-shear-layer through (Wyganski, 1981; Ho and Huerre, 1994):

$$
\theta=\int_{-0.05 L}^{0.05 L} \frac{\rho(y) U(y)-\rho_{1} U_{1}}{\rho_{2} U_{2}-\rho_{1} U_{1}}\left(1-\frac{U(y)-U_{1}}{U_{2}-U_{1}}\right) d y
$$

Where $-0.05 L$ and $0.05 L$ correspond to the upper and lower stream-normal bounds of the domain.

The displacement-thickness represents the mass deficit in a shear layer. Its calculation for boundary layers and free shear layers is based on the following equations, respectively (White, 1992; Wyganski, 1981):

$$
\begin{gathered}
\delta^{*}=\int_{0}^{\delta_{99 \%}}\left(1-\frac{\rho(y) U(y)}{\rho_{f} U_{f}}\right) d y \\
\delta^{*}=\int_{-\infty}^{\infty}\left(1-\frac{\rho(y) U(y)-\rho_{1} U_{1}}{\rho_{2} U_{2}-\rho_{1} U_{1}}\right) d y
\end{gathered}
$$

The shape-factor is defined as the ratio of the displacement-thickness to the momentum-thickness:

$$
H=\frac{\delta^{*}}{\theta}
$$

A shape factor of $H=2.6$ corresponds to a Blasius boundary layer.

The total circulation inside a region of flow is determined using the spanwisevorticity as follows:

$$
\Gamma=\iint_{S} \omega_{z} d A
$$

\section{B.6 Spectral Analysis}

In many instances the relevant frequencies and wavenumbers in the flow-field need to be identified to characterize the stability of the flow. For continuous data a Fourier transform can be used for a signal in time or space to identify the distribution of 
frequencies or wavenumbers respectively at a location in the domain. However, in these numerical simulations flow parameters $(\mathrm{U}, \mathrm{V}, \mathrm{W}, \mathrm{P})$ are sampled discretely in time and space and therefore a discrete Fourier transform is required to study these signals. The fast Fourier transform algorithm performs the discrete Fourier transform in a time-efficient manner for a set of $2^{N}$ discrete points (Rao et al., 2010). A total of 1024 timesteps with a timestep size of $\Delta t=150 \mu s$ were analyzed using FFT because: (a) it is sufficient to resolve the highest frequency signals expected to occur in the simulation; (b) it provides a sufficient number of timesteps to resolve 100 cycles of vortex-shedding; (c) it is an exact power of base 2 required for the FFT.

\section{B.7 Linear Stability Analysis}

A linear stability analysis using the Orr-Sommerfeld equation is performed to compare trends in the numerical results for amplified frequencies with the theoretical prediction for a given baseflow profile. The equations governing the temporal growth of perturbations are solved numerically following linear stability theory, which assumes a perturbation in the form of a two-dimensional wave with a real wavenumber and complex phase velocity. For a perturbation to grow in time, the imaginary component of the complex phase velocity must be positive. A hybrid spectral collocation method based on Chebychev polynomials, described by Schmid and Henningson (2001), is used to calculate the eigenvalues of the Orr-Sommerfeld equation for the computed time-averaged velocity profiles obtained in the numerical study. By solving for the phase velocity and amplification rate over a large wavenumber range (corresponding to frequencies between 10 and $1000 \mathrm{~Hz}$ ), the perturbation frequency yielding the highest amplification rate to shear layer instability modes is predicted. 


\section{B.8 Grid Convergence Study}

A grid convergence study was performed to verify the fidelity of these simulations. The streamwise and wall-normal spacing were proportionally reduced creating a course, medium and fine grid simulation with streamwise and wall-normal spacings of $\left(\Delta x^{+}=17.0, \Delta y^{+}=4.3\right),\left(\Delta x^{+}=11.3, \Delta y^{+}=2.9\right)$, and $\left(\Delta x^{+}=8.5, \Delta y^{+}=2.1\right)$ respectively. These simulations were ran for 20,000 timesteps until statistical steady state is reached. The shed vortices were studied and the Strouhal number of the
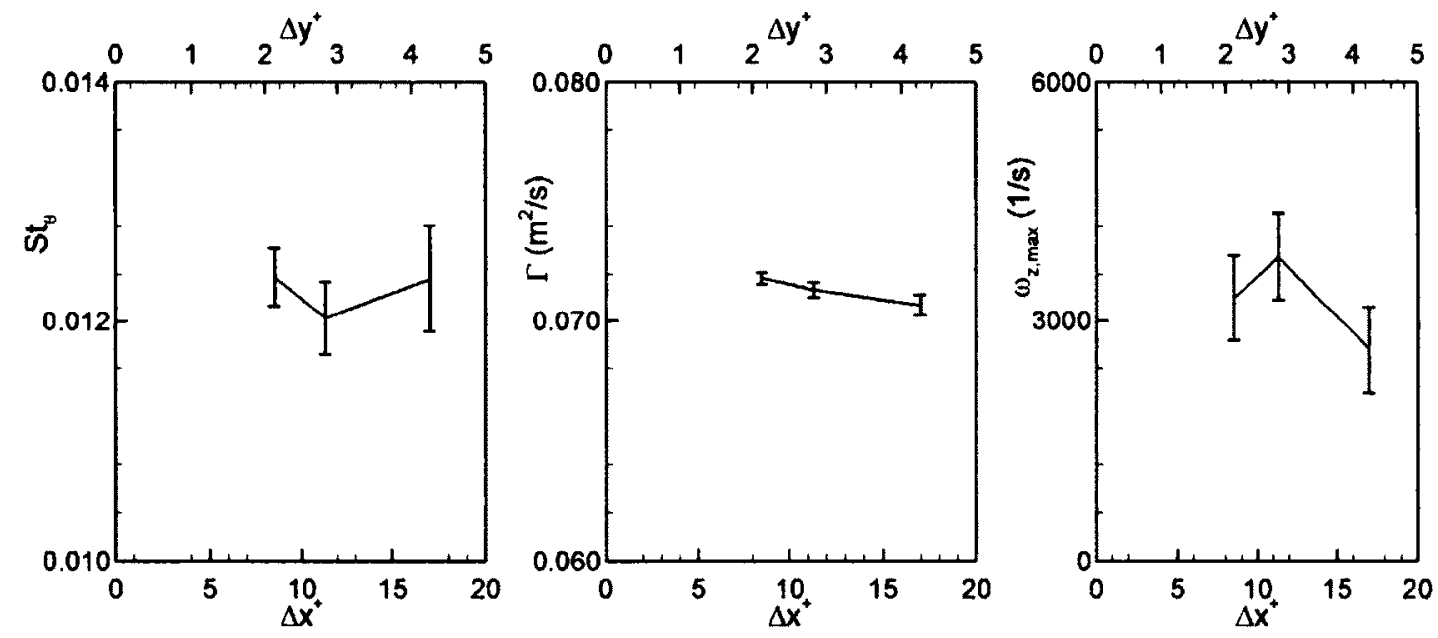

Figure B.1: Comparison of time-mean and perturbation velocity profiles in the $\operatorname{Re}_{\theta}=320, R=1.88$ test cases at two wall proximities.

vortex-shedding, the circulation and maximum vorticity enclosed in each vortex was studied. These quantities were determined by considering vortices as they enter a region of flow enclosed by a "box", similar to the procedure described in Section 4.3. These results are shown in Figure B.1 where the quantities are plotted against streamwise and wall-normal node spacings in wall units. The study shows close agreement between the Strouhal number and the total circulation enclosed within the region of flow. There is some disparity in the maximum vorticity magnitude enclosed in the box, especially for the mid-sized grids. However, Figure B.1 shows that all three 
quantities are within the uncertainty associated with shedding, suggesting adequate convergence. 